\title{
Introduction of Fluorine and Fluorine-Containing Functional Groups
}

\section{Citation}

Liang, Theresa, Constanze N. Neumann, and Tobias Ritter. 2013. Introduction of Fluorine and Fluorine-Containing Functional Groups. Angewandte Chemie International Edition 52, no. 32: $8214-8264$.

\section{Published Version}

doi:10.1002/anie.201206566

\section{Permanent link}

http://nrs.harvard.edu/urn-3:HUL.InstRepos:12336403

\section{Terms of Use}

This article was downloaded from Harvard University's DASH repository, and is made available under the terms and conditions applicable to Open Access Policy Articles, as set forth at http:// nrs.harvard.edu/urn-3:HUL.InstRepos:dash.current.terms-of-use\#OAP

\section{Share Your Story}

The Harvard community has made this article openly available.

Please share how this access benefits you. Submit a story.

Accessibility 


\section{Introduction of Fluorine and Fluorine-Containing Functional Groups}

Theresa Liang, Constanze N. Neumann, and Tobias Ritter*

\section{Keywords:}

$\mathrm{C}-\mathrm{H}$ functionalization fluorine catalysis

trifluoromethylation transition metals

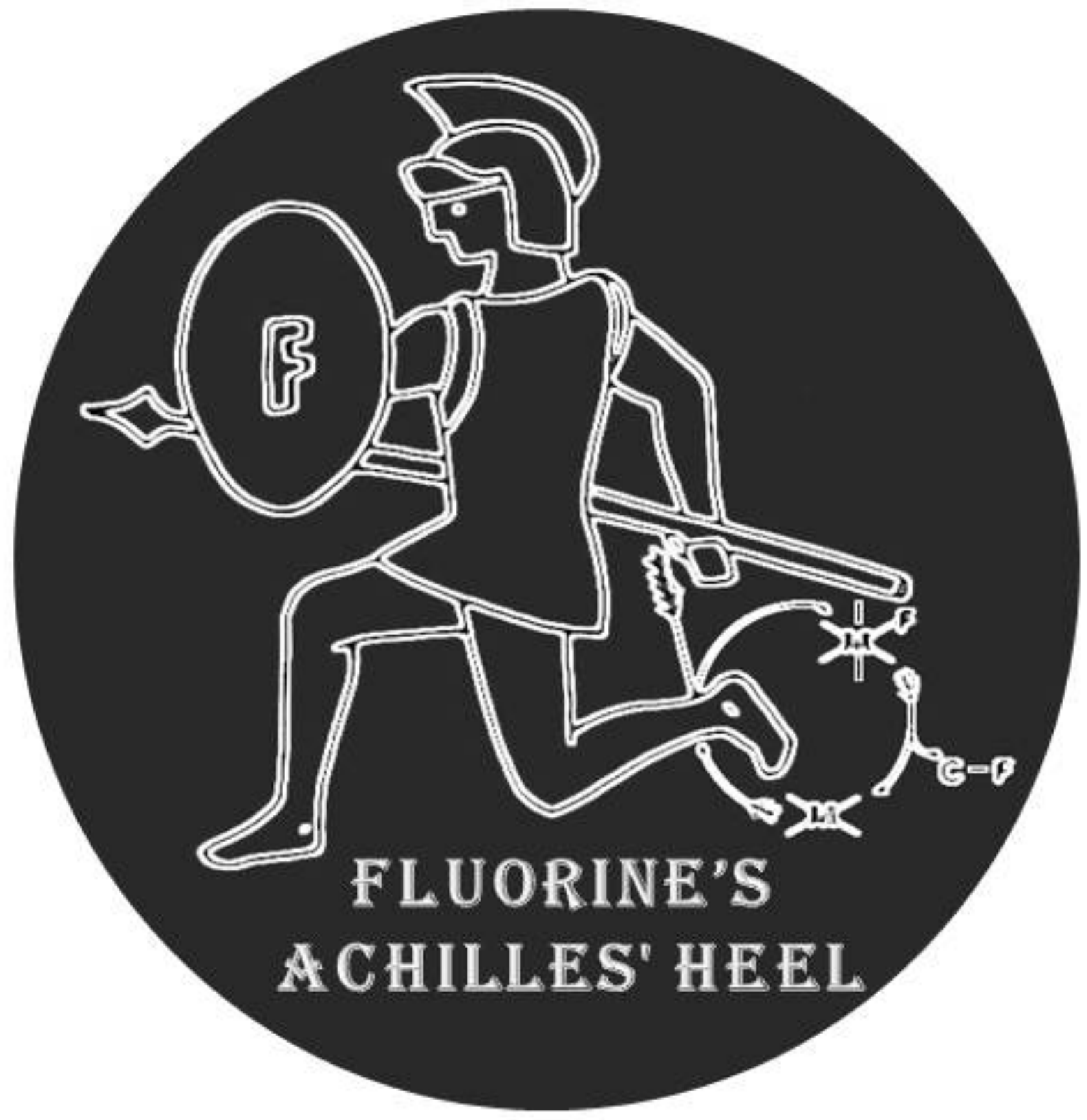




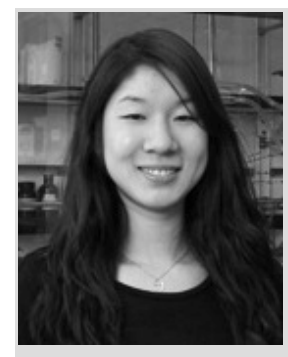

Theresa was born in 1985 in San Jose, California and received her undergraduate education at University of California, Berkeley pursuing research under the mentorship of Prof. Richmond Sarpong in total synthesis. After UC Berkeley, she moved from sunny California to Harvard University and obtained her PhD in 2012 working with Prof. Tobias Ritter on the trifluoromethoxylation of aryl nucleophiles and fluorination of complex natural products.

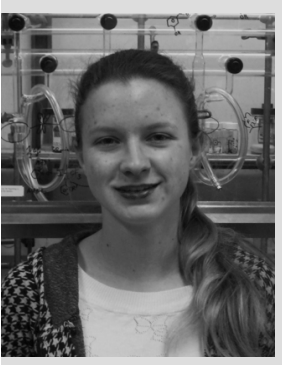

Constanze was born in 1988 in Fürth, Germany and received her MChem at the University of Oxford. She conducted undergraduate research with Prof. Timothy J. Donohoe and then moved to Harvard University where she is pursuing doctoral studies in the group of Prof. Tobias Ritter. Constanze's PhD work revolves around the development of new methods for the $\mathrm{C}-\mathrm{H}$ functionalization of arenes.

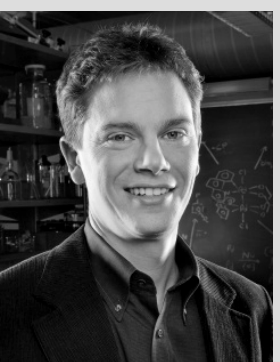

Tobias was born in 1975 in Lübeck Germany. He received his undergraduate education in Braunschweig, Germany, Bordeaux, France, Lausanne, Switzerland, and Stanford, US, and received a master of science from Braunschweig University in 1999. He has performed undergraduate research with Prof. Barry $M$. Trost at Stanford, obtained his PhD working with Prof. Erick M. Carreira at ETH Zurich in 2004, and was a postdoc with Prof. Robert H. Grubbs at Caltech. In 2006, Tobias was appointed as Assistant Professor in the Department of Chemistry and Chemical Biology at Harvard and promoted to Associate Professor in 2010. His research program is based on synthetic organic and organometallic chemistry. The Ritter lab currently focuses on fluorination chemistry for late-stage functionalization of complex natural and unnatural products.

\section{Introduction}

Fluorination chemistry has been developed for more than 100 years with the first examples of nucleophilic and electrophilic fluorination reactions reported in the second half of the 19th century; ${ }^{[1]}$ yet still today, significant challenges in fluorination chemistry remain. Current interest in fluorination chemistry is largely a consequence of the properties that fluorine substitution can impart on molecules, such as pharmaceuticals, ${ }^{[2]}$ agrochemicals, ${ }^{[3]}$ materials, ${ }^{[4]}$ and radiotracers for positron emission tomography (PET) ${ }^{[5]}$ Despite longstanding appreciation of fluorine's utility, fluorination methods still lack generality, practicality, and predictability. Carbon-fluorine bond formation is a challenging chemical transformation largely due to fluorine's high electronegativity and the high hydration energy of fluoride anion. ${ }^{[6]}$ In Nature, haloperoxidase enzymes ${ }^{[7]}$ give rise to thousands of organochlorides and organobromides ${ }^{[8]}$ but no fluoroperoxidase enzyme has been identified. Despite fluorine being the 13th most abundant element in the Earth crust, ${ }^{[9]}$ only a handful of natural biosynthesized organofluorides ${ }^{[9]}$ are known. ${ }^{[10]}$
A few decades ago, the development of several fluorinating reagents such as Selectfluor ${ }^{\mathbb{B}[11]}$ and DAST (diethylaminosulfur trifluoride) ${ }^{[12]}$ resulted in fast development of new fluorination methods. Within the past ten years, a similar leap in fluorination chemistry has occurred, which we ascribe to increased efforts towards catalytic methods for fluorine incorporation. The merger of fluorination chemistry and synthetic organic chemistry-considered separate fields for a long time- has resulted in the recent advances that constitute the focus of this review. Traditional fluorination reactions $^{[13]}$ and their relevance to modern developments are discussed briefly in order to put modern fluorination chemistry ${ }^{[14]}$ into perspective. In addition to $\mathrm{C}-\mathrm{F}$ bond formation chemistry, methods for the introduction of fluorinated functional groups, which exhibit significantly different reactivity than their non-fluorinated analogs, are also presented. For example, while the trifluoromethyl group is formally a fluorine-substituted methyl group, its reactivity is significantly different from that of a methyl group, and it should thus be considered its own functional group. We attempt to cover the existing strategies in the field of fluorination published before 2013 under the aforementioned guidelines, and regret that we were unable to include a more complete view of the field due to editorial constraints.

\section{Fluorination}

Fluorine can provide many beneficial properties when incorporated into a molecule. Modulation of the $\mathrm{pK}_{\mathrm{a}} \mathrm{H}$ of functional groups proximal to fluorine substitution ${ }^{[2 \mathrm{a}, 15]}$ can result in increased membrane penetration at physiological $\mathrm{pH} .{ }^{[16]}$ Fluorinated arenes are more lipophilic than their non-fluorinated counterparts, ${ }^{[15,17]}$ which can be used to advantage in drug development. ${ }^{[2 \mathrm{a}, 2 \mathrm{c}-\mathrm{e}, 2 \mathrm{~g}, \mathrm{~h}]}$ Fluorine is sometimes used as an isostere for hydrogen in medicinal chemistry, but the van der Waals radius of fluorine is more similar to oxygen $(1.47 \AA$ for fluorine versus $1.52 \AA$ for oxygen and $1.20 \AA$ for hydrogen). ${ }^{[18]}$ Fluorinated compounds can be strategically used as transition state inhibitors. ${ }^{[2 \mathrm{c}]}$

The high electronegativity of fluorine contributes to the high carbon-fluorine bond strength due to coulombic attraction between carbon and fluorine due to the polarized covalent bond; the large bond polarization ${ }^{[2 f, 19]}$ results in attractive interactions of the $\mathrm{C}-\mathrm{F}$ fragment with hydrogen bond donors, ${ }^{[20]}$ other fluorinated compounds, ${ }^{[4 \mathrm{c}, 4 \mathrm{~d}, 4 \mathrm{~h}, 21]}$ polar functional groups such as carbonyls, ${ }^{[22]}$ and hydrophobic moieties. ${ }^{[23]}$ Fluorinated molecules can show increased binding affinity to proteins ${ }^{[22-23,23 c, 24]}$ likely due to attractive polar interactions; ${ }^{[25]}$ however, in many cases this phenomenon is empirically observed and rationalized ex post facto and is difficult to predict or design a priori. Most fluorinated compounds, but not all, ${ }^{[26]}$ also exhibit increased metabolic stability by impeding undesired oxidative metabolism pathways. ${ }^{[27]}$

\subsection{Electrophilic fluorination}

Most electrophilic fluorinating reagents are ultimately derived from fluorine gas, the strongest elemental oxidant known, synthesized by electrolysis of potassium bifluoride in hydrogen fluoride. ${ }^{[28]}$ Electrophilic fluorination reactions with highly oxidizing fluorinating reagents ${ }^{[29]}$ such as fluorine gas, hypofluorites 
fluoroxysulfates, and perchloryl fluoride are challenging to perform due to the high reactivity of the reagents. Xenon difluoride was developed as a more stable electrophilic fluorination source, but its high oxidation potential still limits the functional group tolerance of this reagent. ${ }^{[13 \mathrm{e}]}$ The development of crystalline, benchtop-stable fluorinating reagents such as $N$-fluorobis(phenyl)sulfonimide $(\mathrm{NFSI}){ }^{[30]}$ and related analogs,${ }^{[30-31]} \mathrm{N}$-fluoropyridinium salts, ${ }^{[32]}$ and 1-chloromethyl-4-fluoro-1,4-diazoniabicyclo[2.2.2]octane bis(tetrafluoroborate) (Selectfluor $\left.{ }^{\mathbb{Q}}, \mathrm{F}-\mathrm{TEDA}-\mathrm{BF}_{4}\right)^{[11]}$ was crucial for the development of selective, functional group tolerant fluorination methods (Figure 1). Even though $N$-fluoro reagents can formally behave as source of fluoronium cation ("F $\mathrm{F}^{+}$), the $\mathrm{N}-\mathrm{F}$ bonds are polarized toward fluorine, with a partial negative charge on fluorine. Reactions may occur via $\mathrm{S}_{\mathrm{N}} 2$ displacement with nucleophilic attack at fluorine; the $\sigma^{*}{ }_{\mathrm{N}-\mathrm{F}}$ orbitals are sterically inaccessible on nitrogen for nucleophilic attack. Possibly due to the small orbital coefficient of the $\sigma^{*}{ }_{\mathrm{N}-\mathrm{F}}$ on fluorine and the low energy level of the $\sigma^{*}{ }_{\mathrm{N}-\mathrm{F}}$ orbital, other mechanism pathways, such as single-electron transfer, can compete. Often the process by which overall two-electron oxidation proceeds is under discussion; two single-electron transfers or concerted two-electron transfer for fluorination have both been proposed. ${ }^{[33]}$

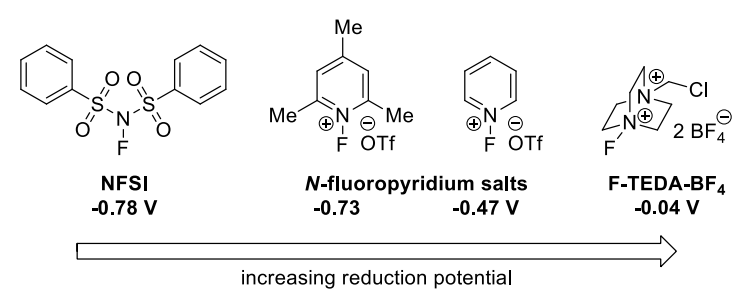

Figure 1. Comparison of the redox potential of crystalline, bench-top stable fluorinating reagents (versus SCE)

\subsubsection{Electrophilic fluorination for the synthesis of fluorinated aromatic carbon centers}

Main group organometallics can be fluorinated electrophilically to afford aryl fluorides. More recently, it was found that transitionmetal organometallics could give rise to aryl fluorides with an enhanced substrate scope. Compared to main group organometallics, transition-metal organometallics can afford aryl fluorides through a wider spectrum of mechanisms, which contributes to their successful use for carbon-fluorine bond formation. Currently, the development of functional group tolerant, direct conversion of aryl $\mathrm{C}-\mathrm{H}$ bonds to the corresponding $\mathrm{C}-\mathrm{F}$ bonds with predictable regioselectivity is a frontier in the field.

Fluorination of arenes via electrophilic aromatic substitution (C$\mathrm{H} \rightarrow \mathrm{C}-\mathrm{F}$ ) is challenging when compared to other halogenations, possibly because the electronegativity of fluorine disfavors the ratelimiting formation of the halocyclohexadienyl cation. Arylmetal reagents including aryltin, ${ }^{[34]}$-mercury, ${ }^{[34 d, 35]}$ lead, ${ }^{[36]}$-lead, ${ }^{[34 b]}$. germanium, ${ }^{[34 f]}$-silicon, ${ }^{[37]}$ and -boron ${ }^{[38]}$ can react with fluorine gas, xenon difluoride, hypofluorites, and fluoroxysulfates to afford fluorinated arenes; however, the substrate scope is limited due to the high reactivity of the reagents, often resulting in unselective fluorination. On the other hand, aryl nucleophiles with more electropositive metals, such as aryllithium reagents, ${ }^{[39]}$ can react with less reactive electrophilic fluorinating reagents, such as $\mathrm{N}$ fluorinated reagents. Such basic nucleophiles can undergo single electron transfer giving rise to protodemetallation byproducts. Fluorination of Grignard reagents with electrophilic $\mathrm{N}$-fluorinated reagents is the most reliable method with simple aryl nucleophiles but is narrow in scope due to the basicity and nucleophilicity of the arylmagnesium reagents. ${ }^{[39 a, 40]}$ Through appropriate choice of solvent and reagents, undesired protodemetallation products can be minimized. ${ }^{[40]}$ More recent work by Meng and Li demonstrated the regioselective para fluorination of anilides with $\mathrm{PhI}(\mathrm{OPiv})_{2}$ and hydrogen fluoride/pyridine. ${ }^{[41]}$

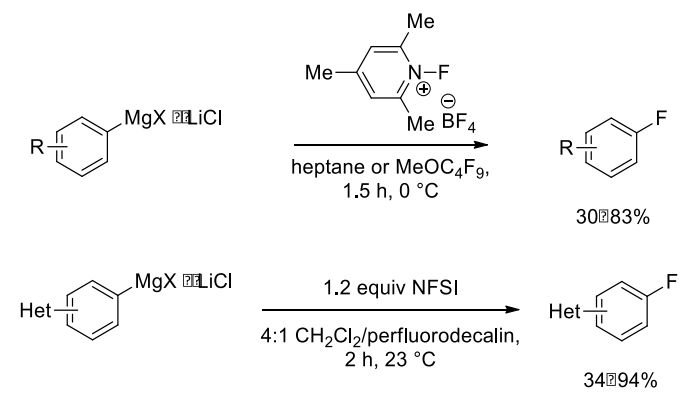

Scheme 1. Synthesis of aryl fluorides from aryl Grignard reagents

Functional-group tolerant and regioselective fluorination with redox active transition metals can proceed through the intermediacy of high-valent organotransition metal fluorides. The metal-carbon bond can be formed via transmetallation or direct $\mathrm{C}-\mathrm{H}$ metalation, followed by oxidation of the metal center with an electrophilic fluorinating reagent. Depending on the reaction conditions, oxidation can result in formation of a monometallic high valent intermediate $^{[42]}$ or a high valent multi-metallic complex. ${ }^{[43]}$

The first transition-metal-catalyzed aromatic fluorination reactions were developed by means of utilizing an orthocoordinating group by Sanford and $\mathrm{Yu}$ (Scheme 2). Direct $\mathrm{C}-\mathrm{H}$ fluorination is desirable, but the necessity of coordinating groups currently limits the structural diversity of the substrates that can be fluorinated. Double fluorination through two subsequent ortho fluorination events was addressed with a weakly coordinating anionic ortho-directing group $N$-perfluorotolylamide as in 1.1, derived from benzoic acid that allows for rapid displacement of the monofluorinated product by the substrate, thus affording high selectivity for monofluorination (Scheme 3).
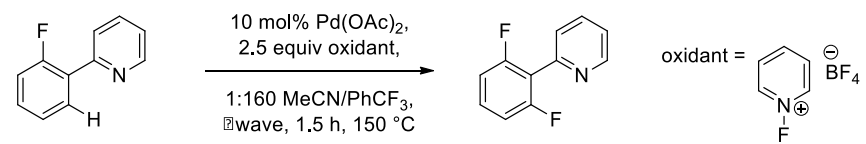

$69 \%$

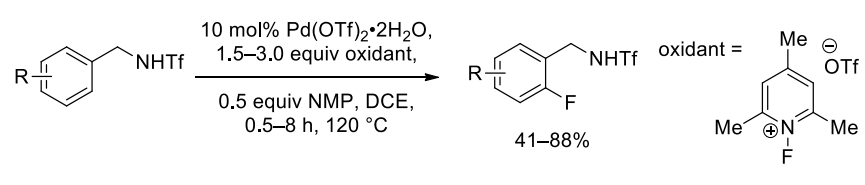

Scheme 2. N-directed Pd-catalyzed fluorination of arenes 


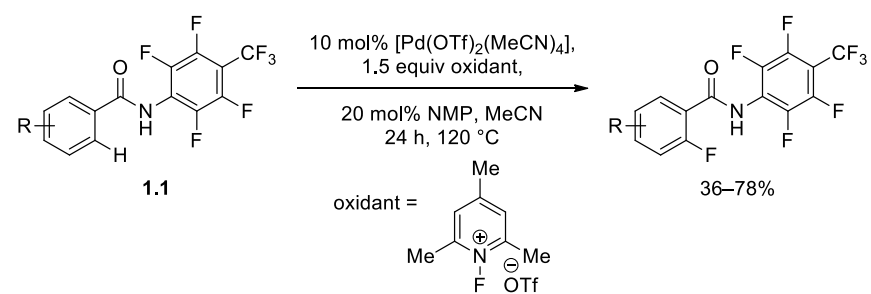

Scheme 3. N-Perfluorotolylamide-directed Pd-catalyzed fluorination of arenes

Arylboronic acids have been used to synthesize $\mathrm{Pd}(\mathrm{II})$ aryl benzoquinoline-sulfonamide complexes $\mathbf{1 . 2}$ that undergo fluorination with F-TEDA-BF 4 in the presence of a variety of functional groups (Scheme 4) ${ }^{[44]}$ however, stoichiometric amounts of Pd complex are needed. Mechanistic studies on the oxidation of the benzoquinoline phenylpyridinesulfonamide-stabilized palladium(II) complex 1.3 with F-TEDA-BF 4 support the presence of a distinct intermediary $\kappa^{3}$-sulfonamide Pd(IV)fluoride species that undergoes reductive elimination to afford aryl carbon-fluorine bonds (Scheme 5). ${ }^{[45]}$
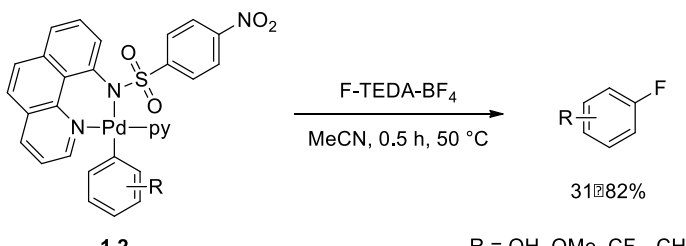

1.2

$\mathrm{R}=\mathrm{OH}, \mathrm{OMe}, \mathrm{CF}_{3}, \mathrm{CHO}$, etc.

Scheme 4. Fluorination of $\mathrm{Pd}(\mathrm{II})$ benzoquinolyl sulfoamide complexes with F-TEDA-BF 4

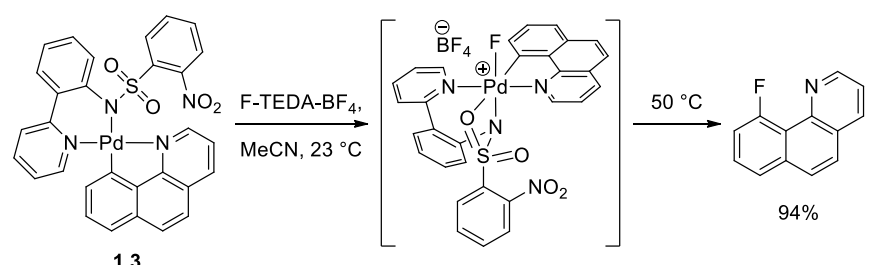

Scheme 5. Reductive elimation from Pd(IV) phenylpyridyl sulfonamide complexes

Following initial work by Tius on silver-mediated fluorination with $\mathrm{XeF}_{2},{ }^{[34 i, 46]}$ a silver-mediated fluorination of functionalized aryl stannanes, ${ }^{[47]}$ boronic acids, ${ }^{[48]}$ and silanes ${ }^{[49]}$ has been developed. These transformation are assumed to proceed through multi-metallic, high-valent silver species, obtained by oxidation of $\mathrm{Ag}(\mathrm{I})$ complexes with F-TEDA-PF 6 followed by reductive elimination. The formation of $\mathrm{C}-\mathrm{F}$ bonds via reductive eliminations is generally a difficult process with requires high temperature to overcome the often high activation barriers. Multi-metallic redox synergy may explain why fluorination of aryl stannanes and boronic acids with silver can occur at $23{ }^{\circ} \mathrm{C}$. Following the discovery of silver-mediated fluorination reactions using a range of arylnucleophiles, a silver-catalyzed fluorination of was developed, which, however, necessitates the use of toxic aryl stannanes. This method displays as of yet the broadest substrate scope and functional group tolerance in the field, including nitrogenous heteroaryl and mesityl nucleophiles, nucleophiles containing electron-rich, electron-poor, electrophilic, and protic functional groups as well as complex natural product-derived substrates (Scheme 6). ${ }^{[50]}$

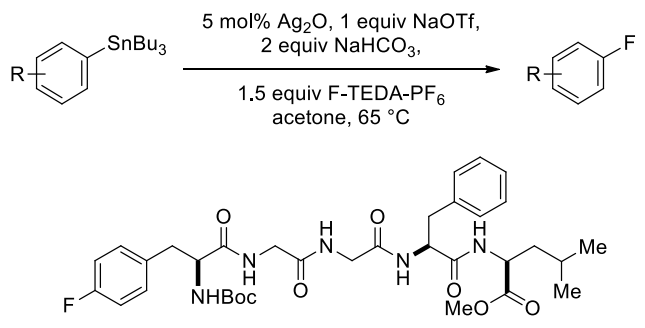

$83 \%$

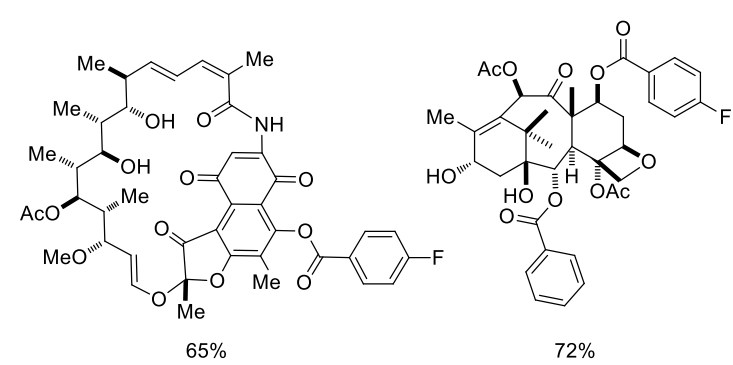

Scheme 6. Ag-catalyzed fluorination of complex aryl stannanes

\subsubsection{Electrophilic fluorination for the synthesis of fluorinated $s p^{3}$ carbon centers}

Nucleophiles employed in aliphatic electrophilic fluorination reactions are often stabilized carbanions, such as those derived from $\beta$-ketocarbonyl compounds. Other methods for the synthesis of aliphatic fluorides can involve fluorination-nucleophile addition cascades across double bonds, ${ }^{[51]}$ which was also reported with alkynes. ${ }^{[52]}$ Enantioselective variants for both fluorination of $\mathrm{C}-\mathrm{H}$ acidic substrates ${ }^{[53]}$ and fluorination-addition cascades have been reported and can be promoted with Lewis acids, organocatalysts, or phase-transfer catalysts. Aliphatic $\mathrm{C}-\mathrm{F}$ bond forming reactions occuring via radical intermediates have been disclosed and can provide a substrate scope complementary to reactions involving two-electron chemistry.

\subsubsection{Electrophilic fluorination of activated methylene and methyne groups}

$\alpha$-Fluorination of carbonyls, $\alpha$-ketocarbonyls, and related carbonyl derivatives with oxidizing fluorinating reagents including gaseous fluorine, ${ }^{[54]}$ alkyl hypofluorite, ${ }^{[55]}$ perchloryl fluoride, ${ }^{[29 b, 56]}$ fluoroxysulfate, ${ }^{[57]}$ and $\mathrm{XeF}_{2}{ }^{[58]}$ generally give undesired $\alpha, \alpha$ difluorinated products in addition to the $\alpha$-monofluorinated products ${ }^{[56 c, 58]}$ Less reactive, more functional group tolerant electrophilic fluorinating reagents such as $N$-fluoropyridinium salts, NFSI, and FTEDA-BF 4 have been used to accomplish selective $\alpha$ monofluorination of carbonyl derivatives. ${ }^{[131,32 \mathrm{a}, 32 \mathrm{~b}, 32 \mathrm{f}, 32 \mathrm{~h}, 33 \mathrm{~b}, 59]}$ The asymmetric $\alpha$-fluorination of carbonyl substrates was explored first with chiral electrophilic fluorinating reagents ${ }^{[31 b, 31 c, 60]}$ and later with chiral catalysts which generate chiral enolate intermediates.

Several methods have exploited the two-point binding of dicarbonyl compounds to chiral Lewis acid complexes to control enantioselective fluorination. Asymmetric fluorination of $\beta$ ketoesters was achieved with titanium-TADDOLate-based catalysts 1.4 and $\mathbf{1 . 5}$ by Togni (Scheme 7), ${ }^{[61]} \mathrm{Cu}(\mathrm{II})-(\text { Scheme } 8)^{[62]}$ and 
Ni(II)-BOX complexes (Scheme 9) ${ }^{[63]}$ by Cahard and Shibata/Toru, respectively, chiral bis(imino)bis(phosphine)ruthenium(II) complex 1.6 by Togni (Scheme 10), ${ }^{[64]}$ and scandium binapthylphosphate complexes by Inanaga. ${ }^{[65]}$ The Ni-catalyzed reaction, using a 10 mol\% catalyst loading, has demonstrated the broadest substrate scope so far, and allows for $\alpha$-fluorination of a variety of $\beta$ ketoesters and $N$-Boc-protected amides ${ }^{[63]}$ in $71-93 \%$ yield and $83-$ $99 \%$ ee, respectively. The catalytic enantioselective $\alpha$-fluorination of $\alpha$-substituted methyl, tert-butyl malonate was accomplished via chiral Lewis acid catalysis with $\mathrm{Zn}(\mathrm{II})$ acetate, $(R, R)-4,6$ dibenzofurandiyl-2,2'-bis(4-phenyloxazoline) ligand, and NFSI. ${ }^{[66]}$ This approach was specifically optimized for the malonate substrate en route to the enantioselective synthesis of fluorinated $\beta$-lactams.

$$
\begin{aligned}
& \mathrm{R}^{1}=\text { alkyl, } \mathrm{Ar} \\
& \mathrm{R}^{2}=\mathrm{Et}, \mathrm{Bn} \text {, etc. } \\
& \mathrm{R}^{3}=\mathrm{Me}
\end{aligned}
$$

Scheme 7. Ti-catalyzed asymmetric $\alpha$-fluorination of $\beta$-ketoesters

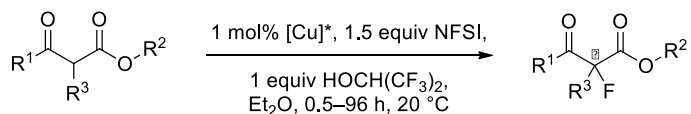

$$
\begin{aligned}
& \mathrm{R}^{1}=\mathrm{alkyl}, \mathrm{Ar} \\
& R^{2}=t B u, B n \text {, etc. } \\
& \mathrm{R}^{3}=\text { alkyl }
\end{aligned}
$$

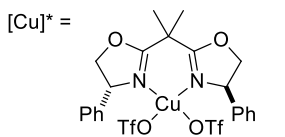

$$
\begin{aligned}
& \text { 80-95\% } \\
& 62-90 \% \text { ee }
\end{aligned}
$$

Scheme 8. Cu-catalyzed asymmetric $\alpha$-fluorination of $\beta$-ketoesters

$$
\begin{aligned}
& \mathrm{R}_{\mathrm{R}^{3}}^{\mathrm{O}} \mathrm{N}_{\mathrm{X}} \cdot \mathrm{R}^{2} \\
& 10 \mathrm{~mol} \% \mathrm{Ni}\left(\mathrm{ClO}_{4}\right)_{2} \cdot 6 \mathrm{H}_{2} \mathrm{O} \text {, } \\
& \underset{4 \mathrm{~A} \mathrm{MS}, \mathrm{CH}_{2} \mathrm{Cl}_{2}, 2-18 \mathrm{~h}, 23^{\circ} \mathrm{C}}{11 \mathrm{~mol} \% \mathrm{~L}^{*}, 1.2 \text { equiv NFSI, }}
\end{aligned}
$$

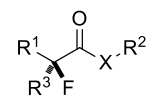

$$
\begin{aligned}
& \mathrm{X}, \mathrm{R}^{1}=\mathrm{O} \text {, carbonyl } \\
& \text { NBoc, Ar } \\
& \mathrm{R}^{2}=t \mathrm{Bu}, \mathrm{Ad}, \mathrm{Bn} \text {, etc } \\
& \text { when } R^{3} \text { then } A r \\
& \mathrm{~L}^{*}=
\end{aligned}
$$

Scheme 9. Ni-catalyzed asymmetric $\alpha$-fluorination of $\beta$-ketoesters and $\mathrm{N}$-Boc oxindoles

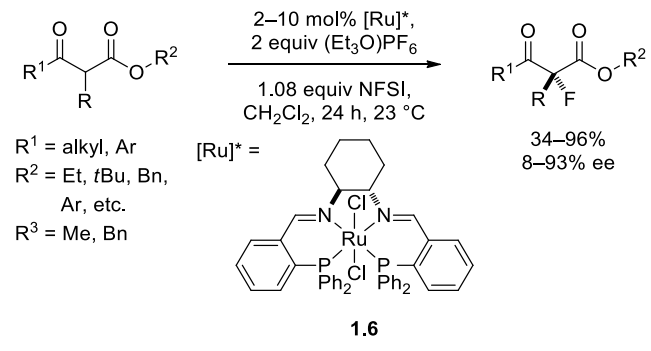

Scheme 10. Ru-catalyzed asymmetric $\alpha$-fluorination of $\beta$-ketoesters
Chiral Pd-BINAP complexes 1.7 and $\mathbf{1 . 8}$ developed by Sodeoka shown in Scheme 11 and Scheme 12 catalyze the enantioselective fluorination of $\alpha$-ketoesters, ${ }^{[67]} \beta$-ketoesters (Scheme 11) ${ }^{[68]} \beta$ ketophosphonates ${ }^{[69]}$ oxindoles (Scheme 12), ${ }^{[70]}$ and $\alpha$-ester lactones/lactams. ${ }^{[71]}$ The use of chiral palladium complexes was particularly successful for the $\alpha$-fluorination of acyclic $\alpha$-ketoesters, cyclic and acyclic tert-butyl $\beta$-ketoester as well as oxindoles $\alpha$ substituted with an electronically diverse range of aryl and alkyl groups. ${ }^{[67-68,70]}$

$$
\begin{aligned}
& 1.5 \text { equiv } \mathrm{NFSI}, \mathrm{EtOH} \\
& 18-72 \mathrm{~h},-20-20^{\circ} \mathrm{C}
\end{aligned}
$$

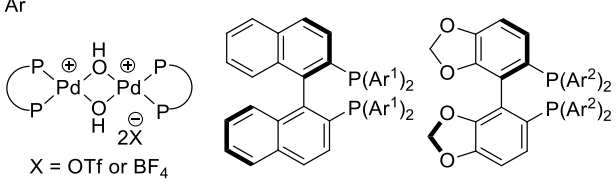

Scheme 11. Pd-catalyzed asymmetric $\alpha$-fluorination of $\beta$-ketoesters

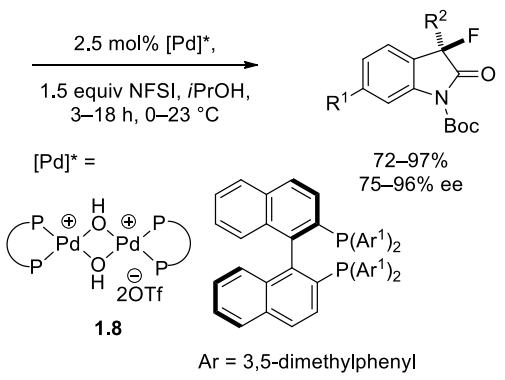

Scheme 12. Pd-catalyzed asymmetric $\alpha$-fluorination of oxindoles.

Organocatalysts provide enantioinduction in the role of chiral fluorinating reagents or through reaction with the substrate to generate chiral nucleophiles.

Cinchona alkaloids have been used to enantioselectively fluorinate nucleophiles in the presence of an achiral fluorinating reagent. Enantioselective fluorination of the activated methyne groups in $\mathbf{1 . 9}$ was accomplished by Takeuchi and co-workers using stoichiometric amounts of cinchona alkaloid derivatives such as 1.10 (Scheme 13). ${ }^{[72]}$ Cinchona alkaloids have also been used to mediate enantioselective $\alpha$-fluorination of silylenol ethers, $\alpha, \alpha-$ cyanoester $\mathrm{C}-\mathrm{H}$ acids, $\beta$-ketoesters, and oxindoles. ${ }^{[73]}$ The methodology was applied to the synthesis of BMS-204352 (Scheme 14) ${ }^{[74]}$ which has also been synthesized utilizing chiral scandium lewis acid catalysts. ${ }^{[75]}$ Enantioselectively $\alpha$-fluorination of $\beta$ ketoesters can be accomplished using cinchona-alkaloid-derived thiourea catalysts. ${ }^{[76]}$ Despite the reduced substrate scope currently tolerated in enantioselective fluorinations with cinchona alkaloids when compared to the Lewis acid-catalyzed fluorinations of $\beta$ ketoesters and oxindoles, chincona-alkaloid-based catalysis has the advantage of not being restricted by the need for a two-point binding site. 


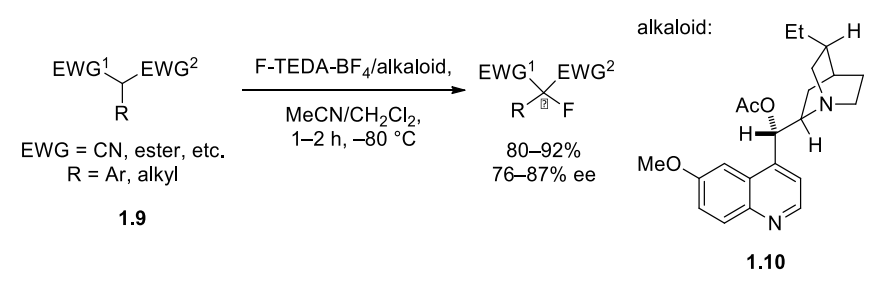

Scheme 13. Enantioselective cinchona-alkaloid-mediated fluorination of $\mathrm{C}-\mathrm{H}$ acidic substrates

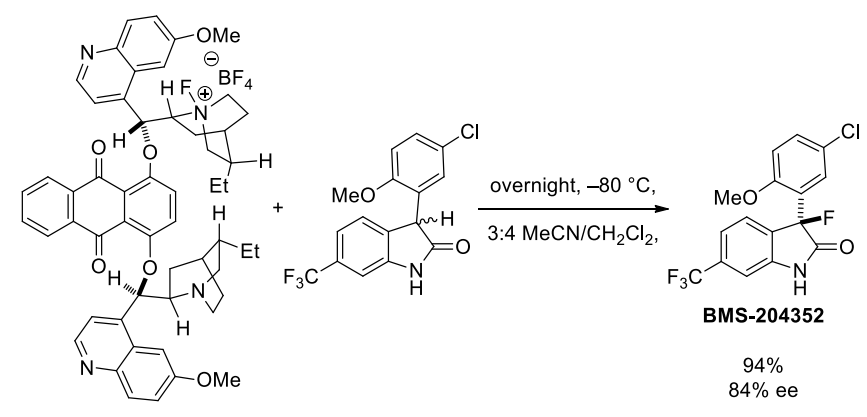

Scheme 14. Enantioselective a-fluorination of oxindoles mediated by $\mathrm{N}$-fluoroammonium salts of cinchona alkaloids

Enantioselective organocatalytic $\alpha$-fluorination of aldehydes was accomplished by Enders, ${ }^{[77]}$ MacMillan (Scheme 15) ${ }^{[78]}$ Jørgensen (Scheme 15), ${ }^{[79]}$ and Barbas (Scheme 16) ${ }^{[80]}$; similarly, enantioselective $\alpha$-fluorination of ketones is possible using enamine catalysis. ${ }^{[81]}$ The aldehyde $\alpha$-fluorination method described by MacMillan demonstrates a broader substrate scope, while the method described by Jørgensen utilizes lower loadings of catalyst and electrophilic fluorinating reagent. Branched aldehydes constitute difficult substrates for enantioselective $\alpha$-fluorination; nonetheless, Barbas reported a promising $98-99 \%$ yield and $45-$ $66 \%$ ee for this class of substrates. The fluorinated aldehyde products are especially useful for the synthesis of enantiopure $\beta$ fluoroamines, which can be obtained by a chiral sulfinylimine condensation, directed reduction sequence of the enantioenriched fluorinated aldehyde. ${ }^{[82]}$

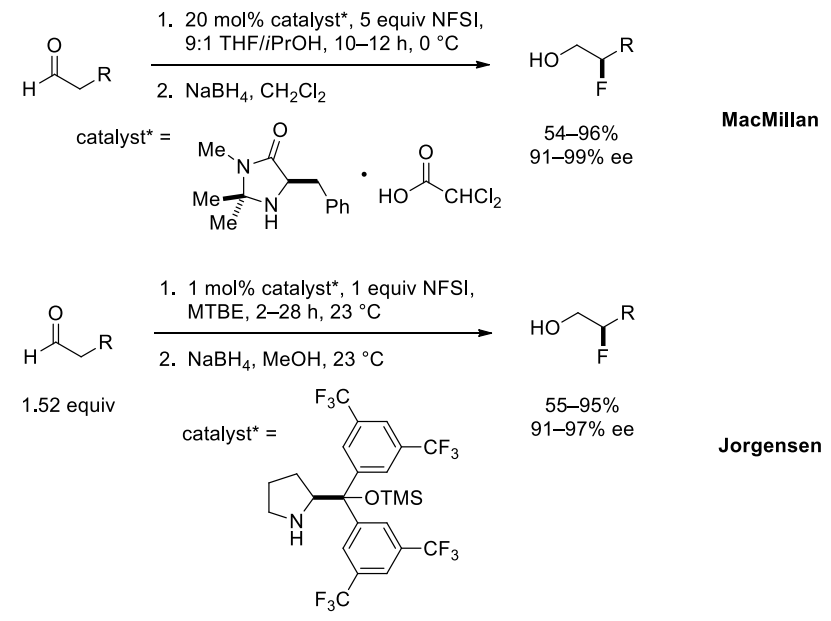

Scheme 15. Organocatalytic asymmetric a-fluorination of aldehydes

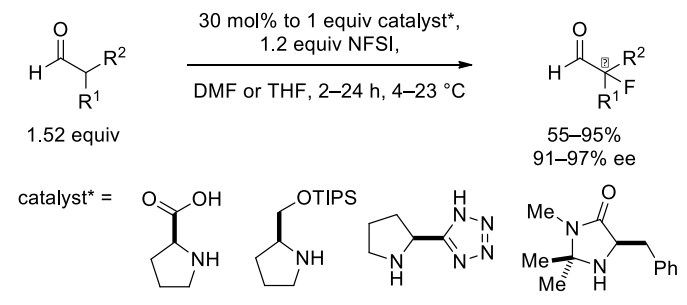

Scheme 16. Organocatalytic asymmetric $\alpha$-fluorination of $\alpha$-branched aldehydes

The enantioselective $\alpha$-fluorination of acid chlorides was accomplished by the Lectka group by combining a palladium catalyst, a chiral nucleophile 1.11, and an alkali metal (Scheme 17). ${ }^{[83]}$ The cinchona alkaloid-based nucleophile reacts with the acid chloride in the presence of the palladium catalyst to generate a chiral zwitterion amide-enolate intermediate. Both catalysts work cooperatively to generate a chiral enolate for fluorination. It is hypothesized that the lithium cation activates NFSI for nucleophilic attack by the chiral enolate through chelation of the sulfonyl oxygen atoms. After fluorination, addition of an amine nucleophile affords the $\alpha$-fluorinated amide product in $>99 \%$ ee and de. Thiourea catalysts derived from cinchona alkaloids can also be used as synergistic bifunctional catalysts for the asymmetric $\alpha$-fluorination of cyclic and acyclic $\beta$-ketoesters with NFSI. ${ }^{[76]}$ The catalyst is able to act as a base (via the quinuclidine moiety) and also as a hydrogen-bond donor (via the thiourea moiety) and is proposed to hydrogen-bond with the substrate and NFSI to afford good enantioselectivity.

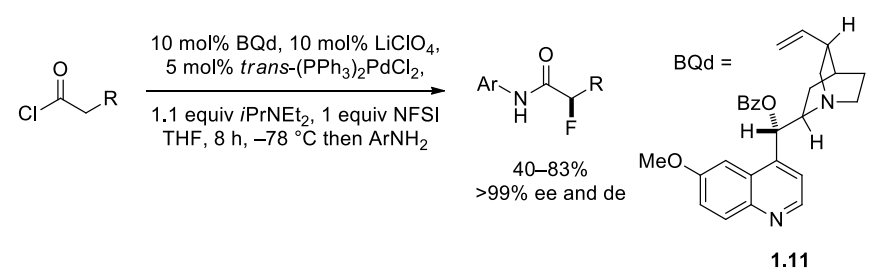

Scheme 17. Organocatalytic asymmetric $\alpha$-fluorination of acid chlorides

Phase-transfer catalysis $(\mathrm{PTC})^{[84]}$ for enantioselective fluorination can offer benefits such as simple operational procedures The formation of chiral tight ion pairs is vital for enantioinduction; thus, nonpolar solvents in which ion pairs remain associated such as dichloromethane and toluene are preferred for PTC. Chiral quaternary ammonium salts have been used as cationic phasetransfer catalysts for enantioselective fluorination of $\beta$-ketoesters with ion pairing between the enolate and chiral ammonium counterion (Scheme 18) ${ }^{[85]}$ Only ee's of up to $69 \%$ have thus far been achieved for the $\alpha$-fluorination of $\beta$-ketoesters; this is likely due to insufficiently tight ion pairing between the chiral ammonium salt and the enolate or a fast background reaction. 

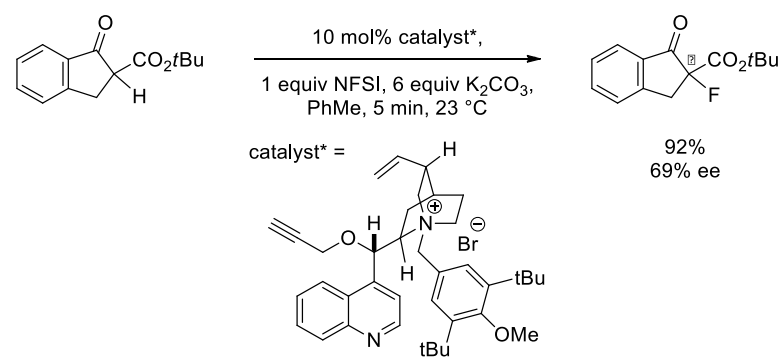

$92 \%$

$69 \%$ ee

Scheme 18. Enantioselective $\alpha$-fluorination of $\beta$-ketoesters with quaternary ammonium salts

A non-traditional approach for the synthesis of chiral allylic and propargylic fluorides described by the Jørgensen group utilizes organocatalysis to facilitate the asymmetric fluorination step followed by olefination or the introduction of a propargyl group. ${ }^{[86]}$ Fluorination of $\alpha$-methylstyrene can occur via oxidative fluorination of the allylic $\mathrm{C}-\mathrm{H}$ bond with a $\mathrm{N}$-fluoropyridinium salt promoted by catalytic amounts of ytterbium(III) triflate. ${ }^{[87]}$ Electrophilic fluorodesilyation of allenyl and allylsilanes was reported to yield propargylic and allylic fluoride products. ${ }^{[88]}$ Gouverneur and coworkers have demonstrated that acyclic secondary allylic fluorides can be prepared via a cross-metathesis-electrophilic fluorodesilyation sequence ${ }^{[89]}$ while chiral tertiary allylic fluorides can be prepared via cinchona alkaloid-mediated electrophilic fluorodesilyation. ${ }^{[90]}$

\subsubsection{Fluorination-nucleophile addition cascades across double bonds}

For activated alkenes like enamines, fluorination can occur without catalysts. For example, fluorocyanation of enamines is hypothesized to occur via electrophilic fluorination of the enamine with NFSI followed by trapping of the iminium intermediate with cyanide (Scheme 19). ${ }^{[51 \mathrm{a}]}$

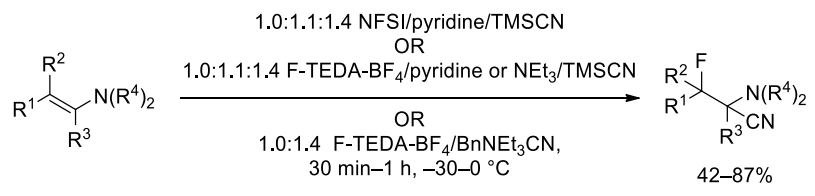

Scheme 19. Fluorocyanation of enamines

With unactivated alkenes, fluorination-nucleophile addition reactions need to be assisted by a catalyst. Both the intramolecular aminofluorination of unactivated alkenes (Scheme 20) ${ }^{[51 b]}$ and the intermolecular aminofluorination of styrenes (Scheme 21$)^{[51 \mathrm{c}]}$ can be facilitated by the use of palladium catalysts as described by Liu. Although both reactions accomplish aminofluorination of alkenes, different approaches were employed and different reaction mechanisms proposed for each case. The intramolecular variant with substrate $\mathbf{1 . 1 2}$ utilizes an iodine(III) oxidant coupled with fluoride to accomplish oxidative fluorination of a complex resulting from intramolecular amino-palladation with an alkene. Intermolecular aminofluorination, on the other hand, is thought to occur via fluoro-palladation involving substrate, NFSI and the active palladium complex, followed by oxidation to a putative $\operatorname{Pd}(\mathrm{IV})$ species, and subsequent reductive elimination to form a carbonnitrogen bond. The formation C-F bonds at $\mathrm{sp}^{3}$ carbon centers by reductive elimination from high-valent transition-metal complexes. ${ }^{[91]}$ has also been investigated with Pt(IV) complexes. ${ }^{[91 \mathrm{c}]}$ Oxidation of stoichiometric alkyl $\mathrm{Au}(\mathrm{I})$ complexes with $\mathrm{XeF}_{2}$ to yield $\mathrm{Au}(\mathrm{III})$ intermediates followed by reductive elimination affords the corresponding aliphatic fluorides. ${ }^{[91 \mathrm{a}]}$ The propensity for $\beta$-hydride elimination from $\mathrm{Au}(\mathrm{III})$ complexes and the need for the strong oxidant $\mathrm{XeF}_{2}$ has limited the substrate scope to mostly $\beta, \beta$ disubstituted alkanes. Additionally, Sanford reported the synthesis of alkyl fluorides by reductive elimination from Pd(IV) complexes. [91c]

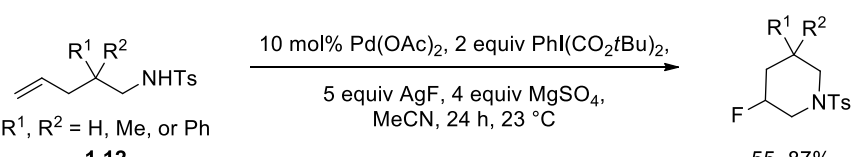

$$
\begin{aligned}
& 1.12 \\
& \text { 55-87\% }
\end{aligned}
$$

Scheme 20. Intramolecular Pd-catalyzed aminofluorocyclization of alkene sulfonamides

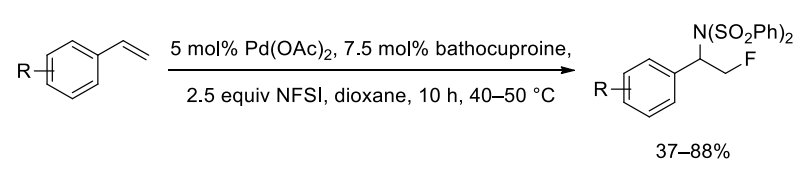

Scheme 21. Intermolecular aminofluorination of styrenes with NFSI

In the enantioselective fluorocyclization of indole substrates mediated or catalyzed by cinchona alkaloids, the enantioselectivity is proposed to arise from the chiral fluorinating reagent generated from the achiral fluorinating reagent and the cinchona alkaloid catalyst $\mathbf{1 . 1 3}$ (Scheme 22). ${ }^{[92]}$

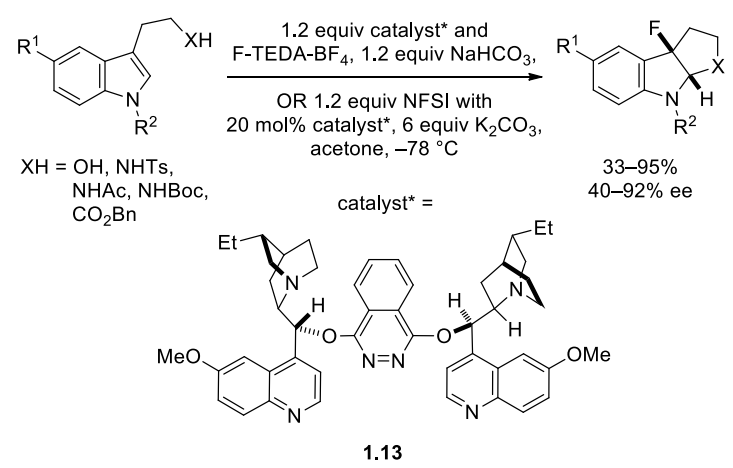

Scheme 22. Enantioselective fluorocyclizations mediated and catalyzed by cinchona alkaloids

An enantioselective fluoro-spiro cyclization reaction via chiral anionic phase-transfer catalysis (PTC) has been realized by Toste through the pairing of chiral anion $\mathbf{1 . 1 4}$ with cationic F-TEDA to provide a source of chiral electrophilic fluorinating reagent (Scheme 23). ${ }^{[93]}$ Because F-TEDA- $\mathrm{BF}_{4}$ is sparingly soluble in the reaction solvent, thus minimizing the background reaction, chiral anion catalysis of the fluorination-spiro cyclization reaction results in enantiomeric excess of up to $96 \%$. Similarly, through the use of PTC with chiral anions, enamides can be enantioselectively fluorinated to afford $\alpha$-fluoroimines ${ }^{[94]}$ or $\alpha$-fluoroalkyoxylation products. ${ }^{[95]}$ 

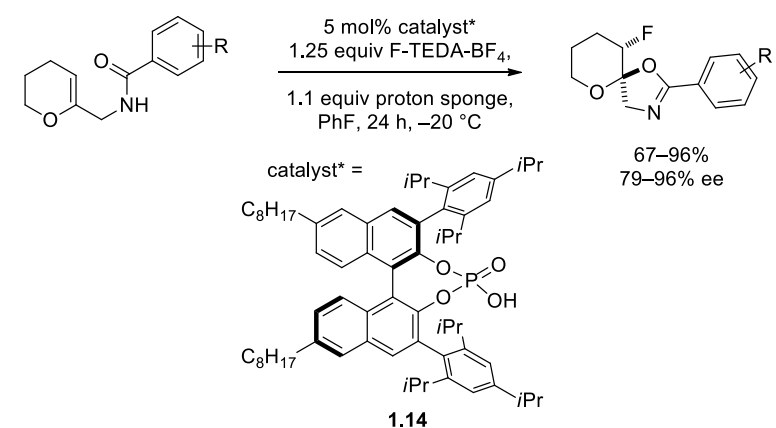

$67-96 \%$ $79-96 \%$ ee

Scheme 23. Enantioselective spiro-cyclizations via chiral anionic PTC

\subsubsection{Transition metal-catalyzed oxidative fluorination of aliphatic $\mathrm{C}-\mathrm{H}$ bonds with fluoride}

Transition metal-catalyzed oxidative fluorination of aliphatic $\mathrm{C}-\mathrm{H}$ bonds using fluoride has been reported for the first time in 2012. ${ }^{[96]}$ The oxidative fluorination of functionalized 8-methyl quinolinyl substrates catalysed by $\mathrm{Pd}(\mathrm{OAc})_{2}$ was reported by Sanford using a hypervalent iodine oxidant in the presence of silver fluoride (Scheme 24). ${ }^{[96 b]}$ This transformation is postulated to occur through high valent palladium fluoride intermediates and is enabled by the strategic concurrent use of $\mathrm{PhI}(\mathrm{OPiv})_{2}$ and $\mathrm{AgF}$. Oxidative $\mathrm{C}-\mathrm{H}$ fluorination of aliphatic substrates with a manganese(III) porphyrin catalyst in the presence of silver fluoride and iodosylbenzene was established by Groves (Scheme 25). ${ }^{[96 \mathrm{a}]} \mathrm{C}-\mathrm{H}$ fluorination is proposed to occur via initial radical $\mathrm{C}-\mathrm{H}$ bond cleavage by the $\mathrm{Mn}(\mathrm{V})$ oxo intermediate, which results from oxidation of the Mn(III) catalyst with PhIO. Radical recombination occurs between the substrate and a Mn(IV) difluoride complex generated from $\mathrm{AgF}$ and a Mn(IV) hydroxide complex. The faster rate of the fluoride for hydroxide ligand exchange on manganese compared to the reaction between the alkyl radical and the Mn(IV) hydroxide complex and the resulting selectivity achieved for fluorination over hydroxylation is remarkable.

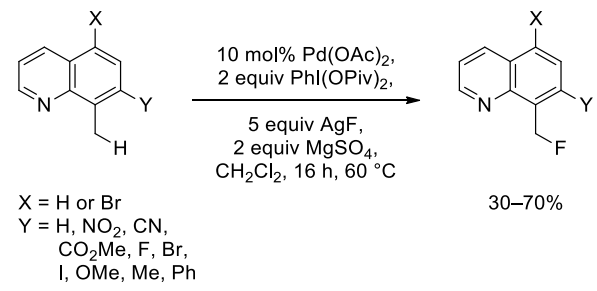

Scheme 24. Pd-catalyzed $\mathrm{N}$-directed oxidative fluorination of $\mathrm{sp}^{3} \mathrm{C}-\mathrm{H}$ bonds with $\mathrm{AgF}$ and $\mathrm{Phl}(\mathrm{OPiv})_{2}$

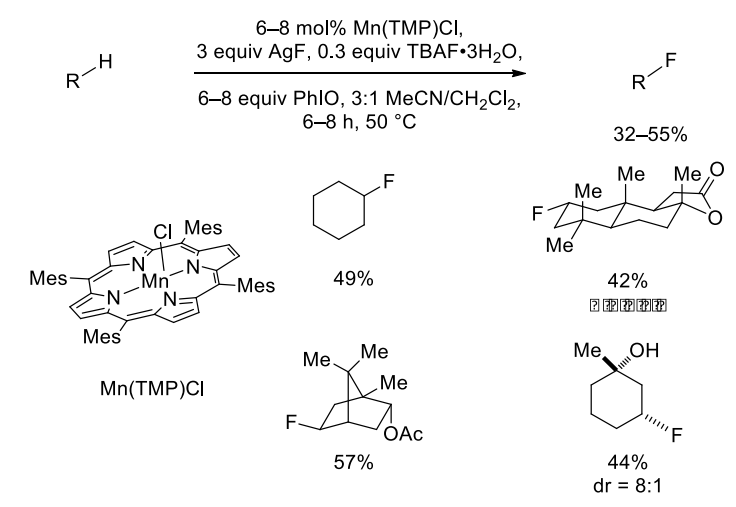

Scheme 25. Mn-catalyzed oxidative aliphatic fluorination with $\mathrm{AgF} / \mathrm{TBAF} \cdot 3 \mathrm{H}_{2} \mathrm{O}$ and iodosylbenzene

\subsubsection{Fluorination via a radical mechanism}

The N-F bonds in electrophilic fluorinating reagents have relatively low bond dissociation energies $(2.84 \mathrm{eV}$ for $N$ fluorosultam) ${ }^{[97]}$ Under either photolysis or thermolysis conditions, a variety of tert-butyl alkylperoxoates afforded the corresponding alkyl fluorides upon treatment with NFSI (Scheme 26). ${ }^{[98]}$ Primary alkyl fluoride formation was not efficient, which supports the mechanism hypothesis of radical intermediates

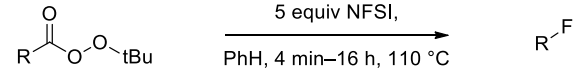

$$
\begin{aligned}
& \mathrm{R}=\mathrm{CH}_{2} \mathrm{OPh}, \quad 24-98 \% \\
& \left(\mathrm{CH}_{2}\right)_{2} \mathrm{CH}_{2} \mathrm{Ph} \text {, } \\
& \text { CHPhMe, } \\
& \text { etc. }
\end{aligned}
$$

Scheme 26. Fluorination of tert-butyl alkylperoxoates with NSFI

Decarboxylative fluorination, first explored by Patrick, ${ }^{[99]}$ was recently reported for secondary and tertiary aliphatic carboxylic acids with F-TEDA-BF 4 by Li via silver catalysis (Scheme 27). ${ }^{[100]}$ This approach is complementary to traditional nucleophilic fluorination reactions with DAST-type reagents. Involvement of Ag-mediated decarboxylation to form an alkyl radical during the reaction was demonstrated, but the detailed mechanism for the key $\mathrm{C}-\mathrm{F}$ bond formation still remains undetermined. Photofluorodecarboxylation of $\alpha$-aryloxyacetic acids or $\alpha$-aryloxy- $\alpha$ fluoroacetic acids was accomplished by treating the acid with $\mathrm{NaOH}$ in the presence of F-TEDA-BF 4 to afford aryl fluoromethylethers or aryl difluoromethylethers, respectively. ${ }^{[101]}$ 


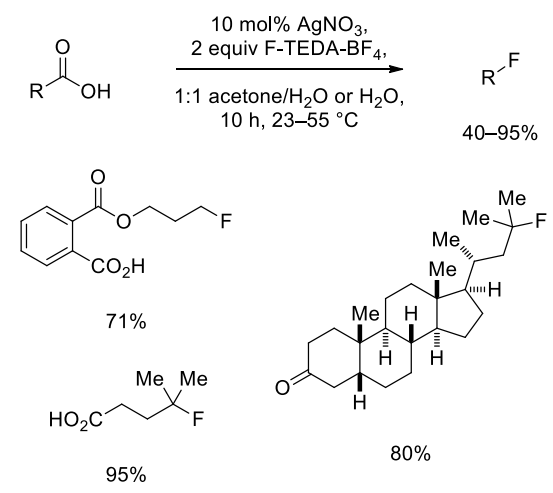

Scheme 27. Silver-catalyzed decarboxylative fluorination of aliphatic carboxylic acids with F-TEDA

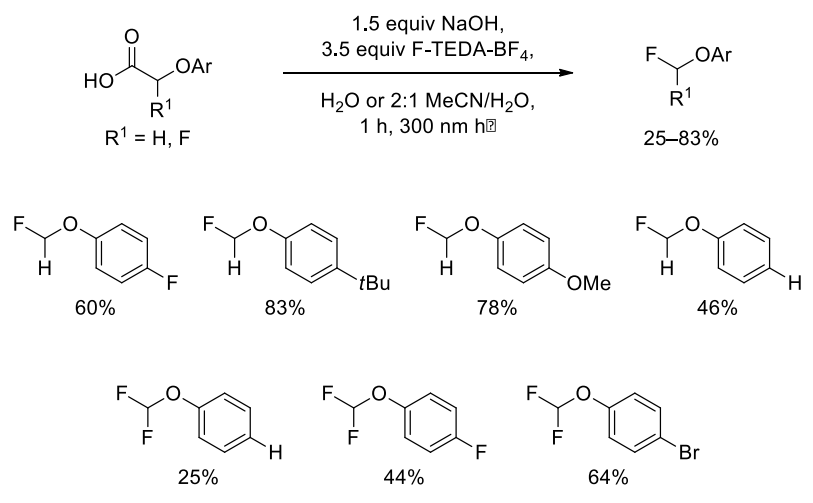

Scheme 28. Photo-decarboxylative fluorination of $\alpha$-aryloxycarboxylic acids with F-TEDA and $\mathrm{NaOH}$

\subsection{Nucleophilic fluorination}

The challenges associated with nucleophilic fluorination ultimately derive from the high electronegativity of fluorine, which contributes to the high kinetic barriers in forming carbon-fluorine bonds despite the thermodynamic driving force of forming the $\mathrm{C}-\mathrm{F}$ bond, the strongest carbon-heteroatom single bond known. The propensity of fluoride to form strong hydrogen bonds can attenuate its nucleophilicity when hydrogen bond donors are present. Rigorous exclusion of potential hydrogen bond donors renders fluoride a good nucleophile, but it also increases its basicity, which often leads to side reactions.

Use of alkali metal fluoride salts is desirable due to low cost especially compared to electrophilic fluorinating reagents. ${ }^{[102]}$ The strong lattice energy of such salts makes them weak nucleophiles and poorly soluble in organic solvents. Crown ethers such as 18crown- 6 can be used in combination with alkali metal fluorides to increase solubility of fluoride salts such as KF which often leads to an increase in reactivity. ${ }^{[103]}$ Aprotic solvents especially polar aprotic solvents ${ }^{[103 b, 104]}$ are preferred for nucleophilic fluorination reactions in order that the nucleophilicity of fluoride anions remains unattenuated by hydrogen bonding interactions; the concomitant increase in fluoride's basicity can however lead to elimination byproducts. The addition of tertiary alcohols such as tertbutano ${ }^{[105]}$ has been shown to maintain fluoride nucleophilicity while diminishing its basicity, thereby reducing undesired byproduct formation. Tetrabutylammonium difluorotriphenylsilicate (TBAT), tetramethylammonium fluoride (TMAF), and tetrabutylammonium fluoride (TBAF, anhydrous TBAF can be synthesized ${ }^{[106]}$ ) are commonly used soluble fluoride sources.

Fluorodeoxygenation of carbon centers commonly requires specialized fluorinating reagents that can accomplish oxygen activation/deoxygenation as well as provide a fluoride source. Various aryl and aminosulfur trifluorides ${ }^{[12 a, 107]}$ and derivatives thereof as well as 2,2-difluoroimidazoline-type reagents ${ }^{[108]}$ participate in fluorodeoxygenation reactions. Several hydrogen fluoride-based reagents ${ }^{[109]}$ have been developed to assist sulfur displacement with fluoride in fluorodesulfurization reactions. Electrochemical fluorination with alkali metal-fluorides has been explored as an approach for the synthesis of organofluorides. ${ }^{[10]}$ Catalysis has enabled many nucleophilic fluorination reactions that are otherwise kinetically difficult to accomplish, such as the fluorination of arenes. Uncatalyzed nucleophilic aromatic substitution with fluoride does not proceed for electron-rich substrates because the rate-determining step is addition of the nucleophile to the arene to form a Meisenheimer-type complex. ${ }^{[11]}$ Additionally, chiral transition-metal and organocatalysts can be used for nucleophilic fluorination reactions to afford enantioenriched fluorinated compounds.

\subsubsection{Nucleophilic fluorination for the synthesis of fluorinated aromatic carbon centers}

$\mathrm{S}_{\mathrm{N}} \mathrm{Ar}$ reactions are currently used on an industrial scale in the Halex (halogen exchange) process, ${ }^{[112]}$ although one or more electron-withdrawing groups on the arene are typically required. Aromatic substitution, catalyzed and non-catalyzed, is, at this point, one of the most direct methods for nucleophilic fluorination with control of regioselectivity. Improvement in the substrate scope is desirable because nucleophilic fluorination is preferred over electrophilic fluorination both on an industrial scale owing to the low cost of alkali metal fluorides and in radiochemical applications due to facile access to ${ }^{18} \mathrm{~F}$-fluoride.

\subsubsection{Traditional nucleophilic fluorination methods with main group elements}

Balz and Schiemann first developed the nucleophilic fluorination of arenes via thermal decomposition of aryl diazonium tetrafluoroborate salts in $1927,{ }^{[113]}$ a process which was improved subsequently. ${ }^{[114]}$ Displacement of chloride in 1-chloro-2,4dinitrobenzene under forcing conditions with anhydrous potassium fluoride (the Halex process) was developed in $1936^{[115]}$ followed by fluorodenitration of arenes, by ipso-attack at the carbon bearing the nitro group (Scheme 29). ${ }^{[16]}$ More recent work in this area has allowed for the fluorination of electron-poor, chloro-, nitro-, or trimethylammoniumarenes with anhydrous tetrabutylammonium fluoride at room temperature in up to $>95 \%$ yield. ${ }^{[117]}$ Aryl fluorides can also be accessed from reaction of aryl bromides with anhydrous tetramethylammonium fluoride; however, the process actually occurs through fluoride trapping of aryne intermediates generated from the elimination of the bromide with the strongly basic anhydrous fluoride and therefore provides a mixture of constitutional isomers (Scheme 30). ${ }^{[18]}$ Other approaches that have been explored involve two-step nucleophilic fluoro-demetallation of toxic organothallium(III) substrates ${ }^{[119]}$ as well as diaryliodonium substrates. ${ }^{[120]}$ Although simple fluorinated arenes can be synthesized with some of the aforementioned reactions, even on 
industrial scale ${ }^{[112]}$ none of these methods are tolerant of many functional groups or exhibit broad substrate scope.

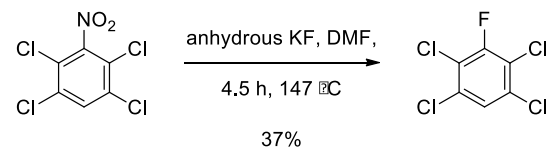

Scheme 29. Nucleophilic aromatic substitution with KF

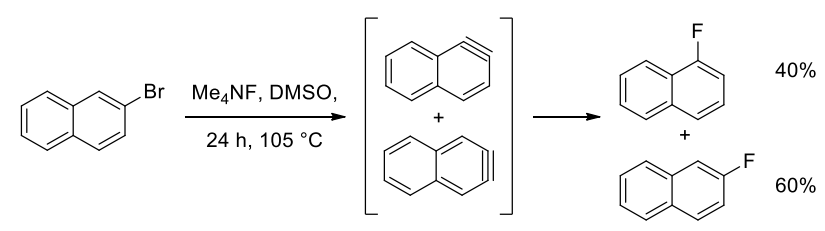

Scheme 30. Fluorination of naphthyl bromide via aryne intermediates

\subsubsection{Transition-metal-catalyzed and -mediated nucleophilic fluorination}

Transition-metal-catalyzed cross-coupling with fluoride as nucleophile has been investigated with late transition-metals such as nickel, copper, ruthenium, rhodium, palladium, iridium, and platinum. ${ }^{[121]}$ Reductive elimination of $\mathrm{C}-\mathrm{F}$ bonds from $\mathrm{Pd}(\mathrm{II})$ is challenging; the difficulty of the reductive elimination step rises with increasing electronegativity of the nucleophilic coupling partner. For Pd(II) complexes stabilized by monodentate ligands, fluoride-bridge dimer complexes are readily formed, from which reductive elimination has never been observed. Cross-coupling fluorination of aryl triflates with fluoride via palladium catalysis (Scheme 31) ${ }^{[121 \mathrm{~g}, 122]}$ was developed by Buchwald and co-workers with the bulky monodentate phosphine ligand $t$ BuBrettPhos, which was crucial for successful $\mathrm{C}-\mathrm{F}$ reductive elimination through a mononuclear, tri-coordinate palladium(II) complex. The fluorination of aryl triflates displays a broad substrate scope and tolerates nucleophilic functional groups not often tolerated in electrophilic fluorination methods due to competing fluorination of these electron rich sites. Protic functional groups are not tolerated under the reaction conditions, and the formation of constitutional isomers, potentially a result of the use of basic fluoride salts, was observed in some cases. ${ }^{[122]}$ Importantly, formation of reduced arenes $(\mathrm{C}-\mathrm{H}$ bond formation instead of $\mathrm{C}-\mathrm{F}$ bond formation) could be suppressed to a minimum, which enables practical purification of the fluoroarene products. Applications for positron emission have been evaluated and are currently limited to the synthesis of radiotracers of low specific activity. ${ }^{[123]}$

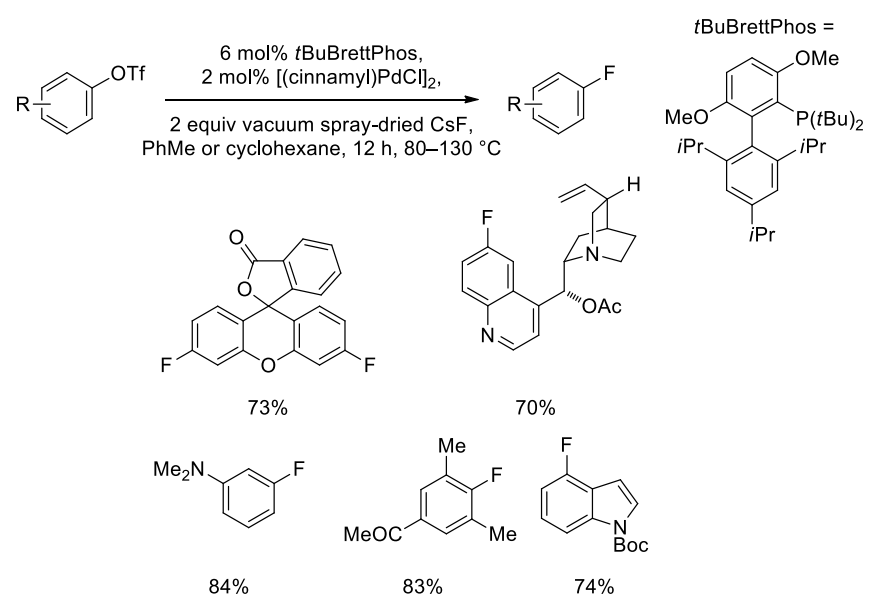

Scheme 31. Pd-catalyzed cross-coupling of aryl triflates with CsF

An oxidative addition - $\mathrm{C}-\mathrm{F}$ reductive elimination sequence was established by the Ribas group via a $\mathrm{Cu}(\mathrm{I})-\mathrm{Cu}(\mathrm{III})$ cycle using a contrived substrate that allowed for isolation of key intermediates (Scheme 32). ${ }^{[121 f]}$ Oxidative addition of copper(I) into the aryl halide supported by the tris(amine) ligand generates a copper(III) complex, which upon ligand metathesis with silver fluoride, gives a copper(III) fluoride complex that undergoes reductive elimination to liberate the aryl fluoride product. Copper-mediated fluorination of electron-rich, electron-poor, as well as hindered aryl iodide substrates was reported by Hartwig, using three equivalents of a copper(I) complex and AgF (Scheme 33). ${ }^{[124]}$ The formation of hydrodehalogenated side products renders purification of the aryl fluoride products challenging.

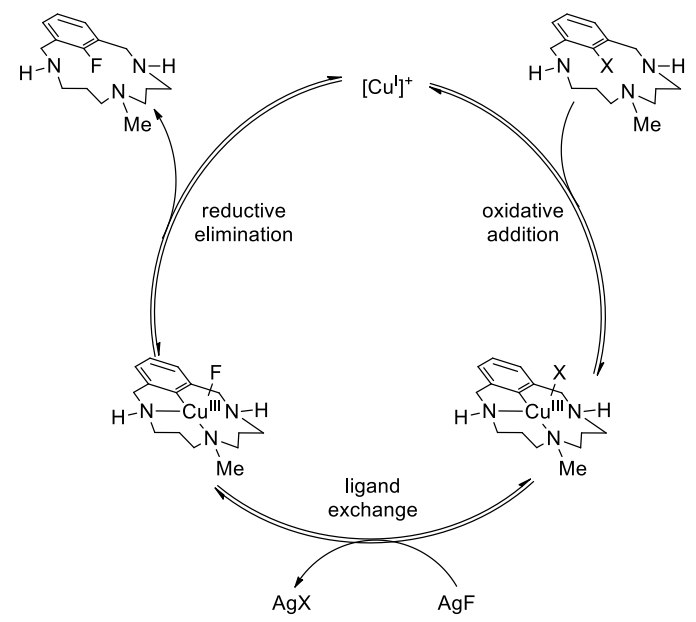

Scheme 32. Cu-catalyzed halide exchange on arenes with AgF 


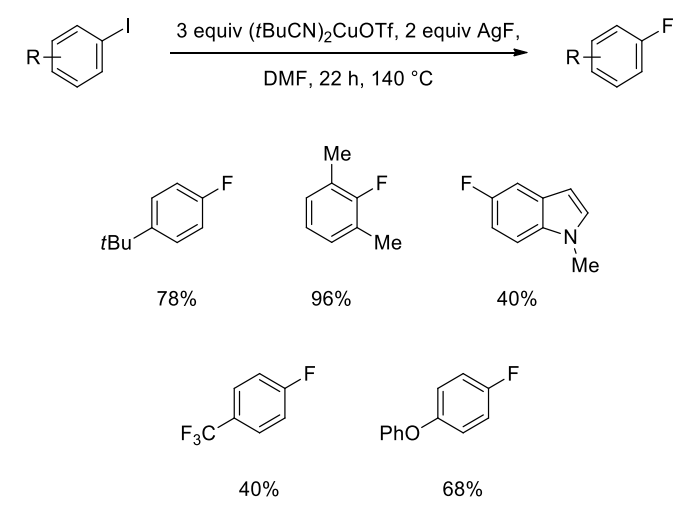

Scheme 33. Cu-mediation fluorination of aryl iodides with AgF

\subsubsection{Nucleophilic deoxyfluorination}

The ability to directly substitute the hydroxy group in phenols with fluoride obviates the need for pre-functionalization of the phenol. Catechol can be monodeoxyfluorinated by oxidation of the catechol to the ortho-quinone followed by nucleophilic deoxofluorination with Deoxo-Fluor ${ }^{\circledR}{ }^{[125]}$ This oxidationfluorination method affords a mixture of ortho-fluorinated phenol isomers. One of the first examples of deoxyfluorination of nitrosubstituted phenol was accomplished with $N, N^{\prime}$-dimethyl-2,2difluoroimidazolidine. ${ }^{[108 a]}$ A general method, as described by our group, for the ipso-deoxyfluorination of phenols was accomplished with the commercially available difluoroimidazoline reagent PhenoFluor $^{\mathrm{TM}}$ and cesium fluoride (Scheme 34). ${ }^{[108 \mathrm{~b}]}$ Electron-poor, -neutral, and -rich aryl fluorides in addition to heteroaromatic fluorides can be synthesized from the corresponding phenol precursors using this method.

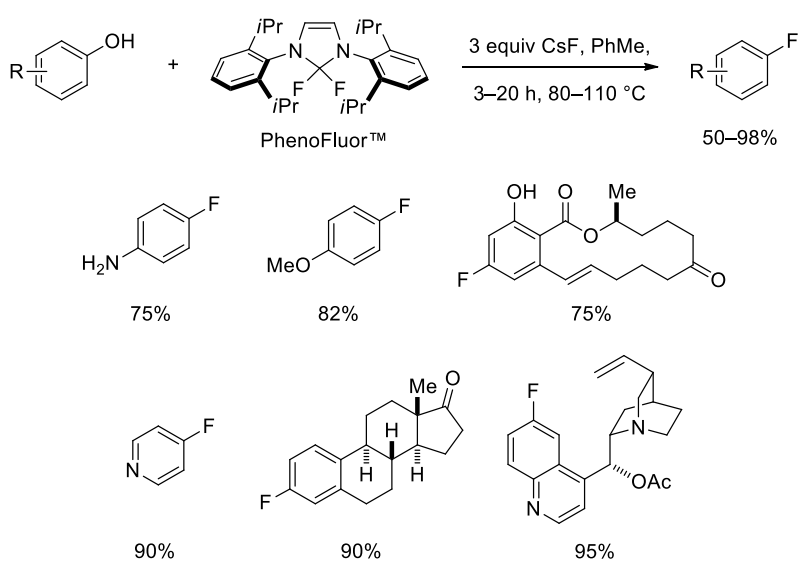

Scheme 34. Deoxyfluorination of phenols with PhenoFluor ${ }^{\text {TM }}$

\subsubsection{Nucleophilic fluorination for the synthesis of fluorinated $s p^{3}$ carbon centers}

Nucleophilic fluorination at primary $\mathrm{sp}^{3}$ carbon centers through appropriate selection of fluoride source, leaving group, and solvent, is well established. ${ }^{[13 a, 103 a, 126]}$ Nucleophilic fluorination at a secondary or tertiary $\mathrm{sp}^{3}$ carbon center is inherently more difficult and no general, functional-group-tolerant method is currently available. Deoxyfluorination with sulphur tetrafluoride or derivatives thereof can afford fluoride from secondary or tertiary carbinols, but competing elimination ${ }^{[12 \mathrm{a}]}$ and rearrangement ${ }^{[12 \mathrm{a}, 127]}$ reactions occur frequently. Halo-fluorination of alkenes were accomplished via treatment of the alkene with $N$-iodosuccinimide and tetraalkylammonium hydrogen fluoride. ${ }^{[128]}$ Nucleophilic opening of epoxides ${ }^{[129]}$ and aziridines ${ }^{[130]}$ with fluoride affords vicinal fluoroalcohols and fluoroamines respectively which have been elaborated to multivicinal multi-fluoroalkanes. ${ }^{[129 \mathrm{~d}, 131]}$

Carbonyl compounds were first converted to geminal difluoromethylene functional groups with sulphur tetrafluoride. ${ }^{[132]}$ The toxicity and volatility of sulfur tetrafluoride led to the development and use of less volatile reagents such as aryl and aminosulfur trifluorides. ${ }^{[12 a,}$ 107] Diethylaminosulfur trifluoride $(\mathrm{DAST})^{[12]}$ has most commonly been used to fluorinate oxygenated (carbonyl, hydroxyl) or sulfur-containing (thiocarbonyl, sulfide) substrates. ${ }^{[107 c, 133]}$ The mechanism of DAST-mediated fluorination is proposed to begin with nucleophilic attack of the alcohol substrate on the sulphur atom of DAST to form an alkoxyaminodifluorosulfane intermediate ${ }^{[134]}$ that is activated for $\mathrm{S}_{\mathrm{N}} 2$ attack by fluoride. In some cases, however, fluorination with DAST affords products consistent with an $\mathrm{S}_{\mathrm{N}} 1$ mechanism. ${ }^{[133 \mathrm{~h}]}$ Additional drawbacks of DAST include moisture sensitivity as well a propensity to explode upon heating. ${ }^{[107 \mathrm{e}]}$

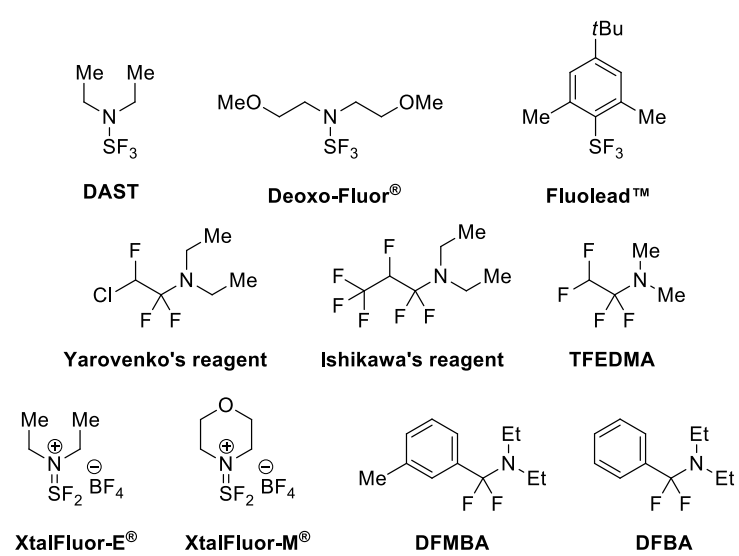

Figure 2. A selection of deoxofluorinating reagents

Besides DAST, deoxyfluorination and dethiofluorination can be accomplished with several different reagents: pyridinium poly(hydrogen fluoride) (Olah's reagent), ${ }^{[109 a, b, 109 d]}$ nitrosonium tetrafluoroborate/pyridinium poly(hydrogen fluoride), ${ }^{[109 \mathrm{c}]}$ triethylamine tris(hydrogen fluorine) $(\mathrm{TREAT} \cdot \mathrm{HF}),{ }^{[109 \mathrm{e}]}$ perfluoro1-butanesulfonyl fluoride (PBSF), ${ }^{[135]}$ sulfonyl fluoride/TREAT•HF mixture (Scheme 35) ${ }^{[136]}$ Yarovenko's reagent, ${ }^{[137]}$ Ishikawa's reagent, ${ }^{[138]}$ TFEDMA, ${ }^{[139]} N, N^{\prime}$-dimethyl-2,2,difluroimidazolidine ${ }^{[108 \mathrm{a}]}$ 4-morpholinosulfur trifluoride, ${ }^{[107 \mathrm{~d},}{ }^{140]}$ Deoxo-Fluor $^{\mathbb{B}}{ }^{[107 f, 141]}$, bromine trifluoride, ${ }^{[142]}$ and 4-tert-butyl-2,6dimethylphenylsulfur trifluoride (Fluolead ${ }^{\mathrm{TM}}$ ). ${ }^{[143]}$ Deoxo-Fluor $^{\mathbb{B}}$ is currently the most commonly used reagent for fluorination reactions and is considered a safer, more thermally stable alternative to DAST but is similarly moisture-sensitive and prone to decomposition to generate toxic HF. Likewise, Olah's reagent exhibits corrosive properties and toxicity due to presence of HF; TREAT $\cdot H F$ is considered to be a less hazardous alternative and is mild enough to be used in borosilicate glassware. 


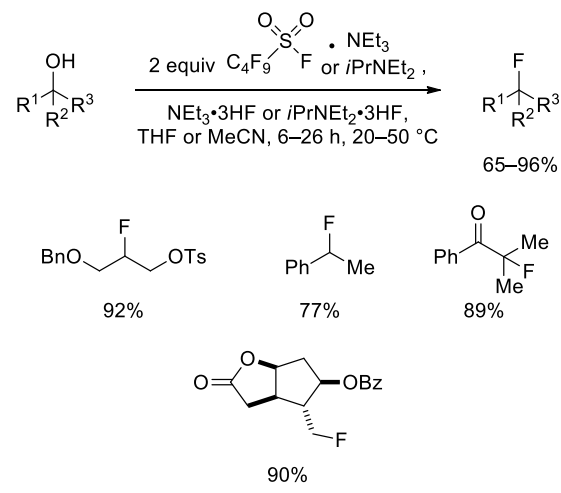

Scheme 35. Deoxyfluorination of various alcohols with perfluorobutanesulfonyl fluoride-trialkylamine adduct and TREAT•HF

Development of the non-explosive, crystalline, less moisturesensitive deoxyfluorinating reagent XtalFluor- $E^{\mathbb{R}}$ (diethylaminodifluorosulfinium tetrafluoroborate) ${ }^{[144]}$ and related reagents ${ }^{[145]}$ has resulted in fluorodeoxygenation with fewer byproducts (Scheme 36). Unlike DAST or Deoxo-Fluor ${ }^{\mathbb{R}}$, fluorination with XtalFluor-type reagents requires the addition of amine hydrogen fluoride such as triethylamine tris(hydrogen fluorine) as a fluoride source because after addition of an alcohol to XtalFluor, the diethylamino group is fully protonated and release the fluoride does not occur. Instead of adding an external fluoride source, addition of DBU can also promote release of fluoride by deprotonation.

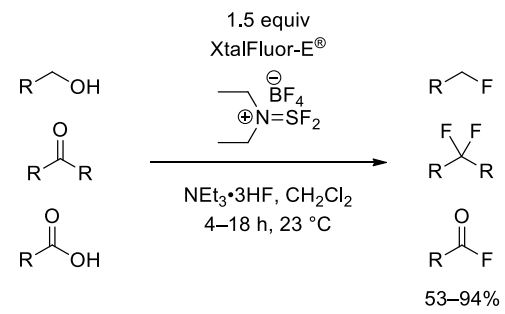

Scheme 36. Deoxyfluorination of alcohols, carbonyls, and carboxylic acids with XtalFluor- $\mathrm{E}^{\circledR}$

The synthesis of propargylic fluorides can be accomplished via deoxyfluorination of propargylic alcohols with $\mathrm{DAST}^{[146]}$ or fluorination of a variety of activated propargylic substrates ${ }^{[147]}$ such as propargylic mesylates or silylethers. Other commonly used deoxofluorinating reagents for the synthesis of propargylic fluorides include $\mathrm{SF}_{4}{ }^{[147 \mathrm{a}, 147 \mathrm{~b}, 148]}$ dialkylaminosulfur trifluorides, ${ }^{[147 \mathrm{c}, \mathrm{d}, 147 \mathrm{f}, \mathrm{g}]}$ Yarovenko's reagent (Figure 2), ${ }^{[149]}$ and Ghosez's reagent $(N, N$ diisopropyl-1-fluoro-2-methylpropenamine). ${ }^{[150]}$ Grée achieved the synthesis of chiral propargylic fluorides through an $\mathrm{S}_{\mathrm{N}} 2$ reaction between chiral, non-racemic propargylic alcohols and fluoride ${ }^{[151]}$ This approach is substrate dependent; many chiral propargylic alcohols give enantiomeric excess-eroded or racemized products through a competing $\mathrm{S}_{\mathrm{N}} 1$ pathway. The majority of syntheses of progarylic fluorides utilize nucleophilic fluorination; Hammond ${ }^{[152]}$ and Gouverneur ${ }^{[88 \mathrm{a}]}$ have investigated electrophilic fluorination routes to propargylic fluorides.

Middleton first reported the dehydroxyfluorination of allylic alcohols with DAST, ${ }^{[146]}$ which was used subsequently to study the fluorination of allylic alcohol substrates containing different substituents and substitution patterns. ${ }^{[153]}$ Temporary complexation of the $\pi$-system of allylic alcohols using a stoichiometric amount of rhenium ${ }^{[154]}$ or iron ${ }^{[155]}$ can prevent allylic transposition during dehyroxyfluorination reactions, a common side reaction. Complexation of chiral racemic allylic alcohols leads to two diastereomeric transition metal complexes, which can be separated and fluorinated stereospecifically. The need for stoichiometric amounts of transition metals, as well as additional protectiondeprotection steps has limited the utility of this approach. Besides DAST, other reagents display reactivity in dehydroxyfluorinations, such as bis(dialkylamino)sulfur difluorides, ${ }^{[146,156]}$ Yarovenko's reagent, ${ }^{[157]}$ Ghosez's reagent, ${ }^{[158]}$ and $\mathrm{IF}_{5}$ in $\mathrm{NEt}_{3} \cdot 3 \mathrm{HF} .{ }^{[159]}$ The reactivity of other activated allylic substrates has also been explored ${ }^{[160]}$ An attractive route to allylic fluorides is the TsujiTrost-type fluoride displacement of a leaving group catalyzed by a transition-metal complex. Because fluoride is a better leaving group than acetate, which is a commonly employed leaving group in TsujiTrost reactions, ${ }^{[161]}$ the selection of an appropriate leaving group proved crucial in the development of this transformation. The selective synthesis of either linear or branched allylic fluorides constitutes an additional challenge ${ }^{[162]}$ in the Tsuji-Trost-type allylic displacement reaction. Doyle reported an enantioselective fluorination of cyclic ${ }^{[163]}$ and acyclic (Scheme 37) ${ }^{[164]}$ allylic chlorides in the presence of a chiral palladium catalyst with branched : linear selectivities of $>20: 1$ for most substrates. A variety of functional groups can be tolerated; currently, long reaction times and an excess of silver fluoride is required for optimum yields. Oxabicyclic alkenes can undergo ring-opening with fluoride and a rhodium catalyst. ${ }^{[165]}$ Secondary and tertiary allylic fluorides can be synthesized from allylic trichloroacetamidates $\mathbf{1 . 1 6}$ under iridium catalysis (Scheme 38$)^{[166]}$ while terminal allylic fluorides can be synthesized from cinnamyl allylic carbonates as reported by Gouverneur and Brown ${ }^{[167]}$ and cinnamyl allylic phosphorothioate esters as reported by $\mathrm{Wu}$, both via palladium catalysis. ${ }^{[168]}$
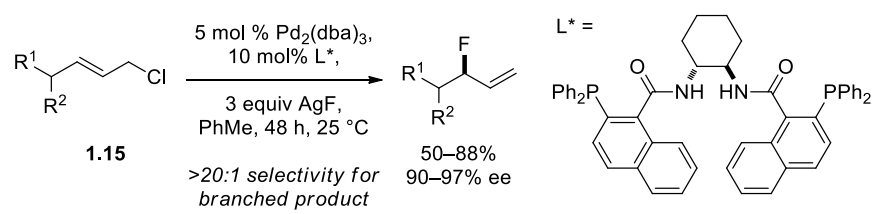

Scheme 37. Pd-catalyzed enantioselective synthesis of branched allylic fluorides

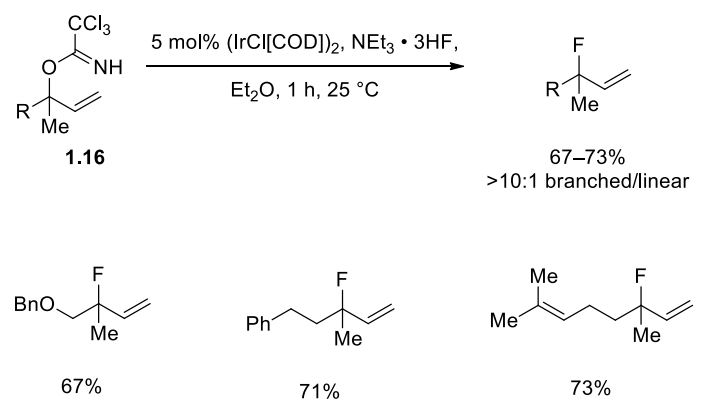

Scheme 38. Ir-catalyzed fluorination of allylic trichloroacetamidates

\subsubsection{Nucleophilic fluorination of olefins and epoxides}


In addition to $S_{N} 2$ reactions, nucleophilic fluorination of olefins through bromofluorination of olefins ${ }^{[169]}$ and epoxide opening with fluoride can provide access to fluorinated secondary or tertiary carbon centers. Asymmetric opening of meso-epoxides with fluoride was first reported by Haufe with stoichiometric amounts of chiral Lewis acid complexes ${ }^{[170]}$ and later with a Co(III)-salen catalyst $\mathbf{1 . 1 7}$ by Doyle and co-workers (Scheme 39) ${ }^{[171]}$ for nonlinear meso-epoxides and terminal epoxides. In situ fluoride release from benzoyl fluoride was employed to suppress background reactions and catalyst inhibition. Mechanistic studies suggest that the rate-determining epoxide opening step proceeds via a bimetallic complex, which led to the design of a more efficient dimeric catalyst containing two linked molecules of the cobalt-salen complex (Scheme 39).

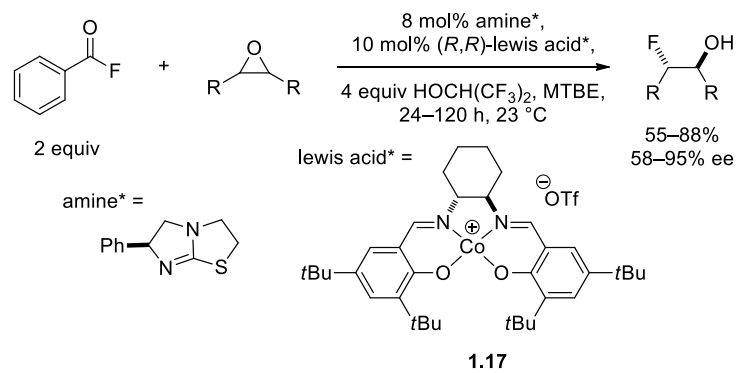

Scheme 39. Co(III)-catalyzed asymmetric opening of meso-epoxides with fluoride

\section{Fluoromethylation}

Fluorinated functional groups such as monofluoromethyl and difluoromethyl groups have been used as oxygen mimics in molecules such as nucleotides, phosphate esters, and sulphate esters. $^{[172]}$

Introduction of fluoromethyl groups via a $\mathrm{S}_{\mathrm{N}} 2$ reaction with fluoromethyl halide reagents is more challenging than methylation with methyl halide reagents because the transition state involves build-up of partial positive charge on the pentacoordinate carbon functionalized with electronegative fluorine. Reactive nucleophiles and fluoromethylating reagents with good leaving groups are thus required for electrophilic fluoromethylation. Difluoromethylation and trifluoromethylation reactions via a $\mathrm{S}_{\mathrm{N}} 2$ mechanism are increasingly impeded by the compounded destabilizing effect of each additional fluorine atom. Nucleophilic fluoromethylation of electrophiles relies on the use of fluoromethide equivalents, typically containing mesomeric stabilizing groups such as sulfones. Many $\alpha$-fluorinated carbanions are kinetically unstable due to 1,1elimination to form carbenes. Although rare, an example of radical fluoromethylation has been reported. ${ }^{[173]}$

\subsection{Electrophilic methods for fluoromethylation}

Fluoromethylation of phenols, thiophenols, as well as of imidazole and indole can be accomplished with chlorofluoromethane as the alkylating reagent as described by $\mathrm{Hu}$ and co-workers. ${ }^{[174]}$ The reaction is postulated to occur via an $\mathrm{S}_{\mathrm{N}} 2$ mechanism, rather than through single electron transfer processes. ${ }^{[174]}$ Monofluoromethylated amines, ethers, and sulfides can be unstable due to the hyperconjugation of the lone pair electrons with the $\sigma^{*}$ C-F orbitals, which results in the elongation of the $\mathrm{C}-\mathrm{F}$ bond and can lead to fluoride extrusion. Fluoromethylsulfonium reagent 2.1, which undergoes fluoromethylation reactions with tertiary amines, imidazoles, phosphines, carbon-based nucleophiles, and even carboxylic and sulfonic acids was developed by Prakash and Olah (Scheme 40). ${ }^{[175]}$ The process by which electrophilic fluoromethyl transfer occurs is currently unclear; both two-electron and one-electron pathways are conceivable. Because reagent $\mathbf{2 . 1}$ is synthesized through chlorofluoromethane alkylation of sodium thiophenolate, fluoromethylation with chlorofluoromethane is more efficient and can afford comparable yields for reactive substrates such as phenols, thiophenols, and imidazoles, ${ }^{[175]}$ Electrophilic fluoromethylation of carbon nucleophiles is limited to methyne nucleophiles containing mesomeric stabilizing groups because for the corresponding methylene nucleophiles, the reaction products are susceptible to hydrogen fluoride elimination. Another fluoromethylating reagent $N$-dimethyl-S-fluoromethyl- $S$-phenylsulfoximinium, developed by Shibata and co-workers, is used for alkylation at oxygen of nucleophiles such as phenols and $\alpha$-carbonyl-enols; the reason for the chemoselectivity is currently under investigation. ${ }^{[176]}$ To the best of our knowledge, electrophilic fluoromethylation of aryl nucleophiles have not yet been reported in the literature.

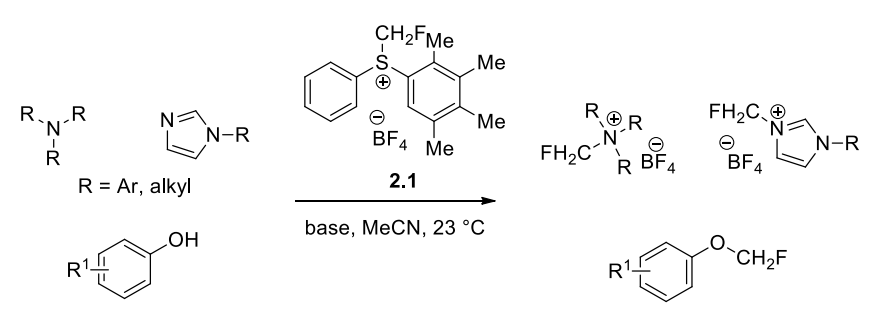

Scheme 40. Monofluoromethylation of various $\mathrm{N}-, \mathrm{O}-, \mathrm{C}$-nucleophiles

Another approach for the synthesis of fluoromethylated ethers involves the oxidative rearrangement of benzyl alcohols induced by $\mathrm{XeF}_{2}{ }^{[177]}$ or aryl difluoro- $\lambda^{3}$-bromane (Scheme 41) ${ }^{[178]}$ through phenyl group participation in the aryl carbon-oxygen bond forming step of aryl $\alpha$-fluoromethyl ether synthesis. Electrophilic fluorination of $O, S$-acetals ${ }^{[179]}$ and $\alpha$-carboxymethyl ethers ${ }^{[99,180]}$ can also afford $\alpha$-fluoromethyl ethers. Relatedly, $N$-fluoropyridium triflate can be used to oxidize the $\mathrm{C}-\mathrm{H}$ bond on the methyl group of methyl sulfide to afford $\alpha$-fluoromethyl sulfides. ${ }^{[181]}$

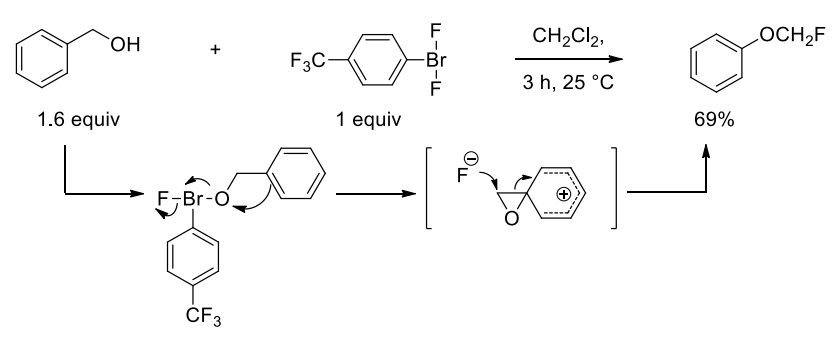

Scheme 41. Oxidative rearrangement of benzyl alcohol to monofluoromethyl phenol with aryl difluoro- $\lambda^{3}$-bromane

\subsection{Nucleophilic methods for fluoromethylation}

The synthesis of $\alpha$-fluoromethyl ethers ${ }^{[182]}$ and sulfides ${ }^{[183]}$ can be accomplished from chloromethyl ethers or thioethers through 
chloride displacement with KF Nucleophilic displacement requires heating in refluxing acetonitrile and starting materials often decompose before displacement occurs. Additionally, nucleophilic fluorinating reagents such as DAST, ${ }^{[184]}$ tetrabutylammonium dihydrogentrifluoride ${ }^{[185]}$ and Deoxo-Fluor ${ }^{\mathbb{B}[141 \mathrm{c}]}$ have been used to induce fluoro-Pummerer rearrangements of sulfides and sulfoxides to yield $\alpha$-fluoromethylsulfides. It should be noted that $\alpha$ fluoromethyl sulfides are easily oxidized to the $\alpha$-fluoromethyl sulfoxide in ambient atmosphere.

The general approach to nucleophilic fluoromethylation involves electrophiles bearing electron-withdrawing groupstabilized fluoromethide equivalents generated by deprotonation of pronucleophiles such as fluorobis(phenylsulfonyl)methane (FBSM) or $\alpha$-fluoro(phenylsulfonyl)methane (FSM). Diasteroselective fluoromethylation of chiral $N$-(tert-butylsulfinyl)aldimines (Scheme $42)^{[186]}$ and ketimines (Scheme 42), ${ }^{[187]}$ chiral $\alpha$-amino $N$-(tertbutylsulfinyl)aldimines ${ }^{[188]}$ have been reported as well as fluoromethylation of aldehydes ${ }^{[189]}$ (followed by a Ritter-type reaction to give $\alpha$-fluoromethylated acetamides ${ }^{[190]}$ ) and the $1,4-$ fluoromethylation of $\alpha, \beta$-unsaturated carbonyls. ${ }^{[191]}$ The use of a chiral auxiliary implies the need for stoichiometric amounts of chiral material, but $>99: 1$ facial selectivity can be obtained in the transformation. Diastereoselectivity is proposed to originate from a closed six-membered metal cation-chelation transition state with the sulfinyl directing group and the $\alpha$-fluoro(phenylsulfonyl)methyl anion. Fluorobenzylation has also been reported with fluorobenzylsulfone pronucleophiles. ${ }^{[192]}$

$$
\begin{gathered}
\begin{array}{c}
\text { 1.05 equiv LHMDS, } \\
1 \text { equiv } \mathrm{PhSO}_{2} \mathrm{CH}_{2} \mathrm{~F} \\
\mathrm{THF}, 40 \mathrm{~min},-78{ }^{\circ} \mathrm{C} ;
\end{array} \\
\begin{array}{c}
\text { 8 equiv } \mathrm{Na}(\mathrm{Hg}) \text { and } \\
\mathrm{Na}_{2} \mathrm{HPO}_{4}, \mathrm{MeOH} \text { then } \\
\text { e equiv } 4 \mathrm{~N} \mathrm{HCl} \text { in dioxane }
\end{array}
\end{gathered}
$$

Scheme 42. Monofluoromethyl pronucleophile addition to chiral $\mathrm{N}$ (tert-butylsulfinyl)aldimines and ketimines

The nucleophilic reactivity of resonance-stabilized fluoromethyl anions have been used in the Mitsunobu reaction of alcohols ${ }^{[193]}$ as well as substitution reactions with alkyl halides ${ }^{[194]}$ en route to monofluoroalkenes. Hydrofluoromethylallylation of alkynes with palladium catalysts ${ }^{[195]}$ affords 3-fluoropropene products. Enantioselective fluoromethylation via transition-metal catalysis, PTC, and organocatalysis have been established with fluoromethyl pronucleophiles, most commonly with FBSM. Analogous to asymmetric allylic alkylation reaction with other nucleophiles with $\mathrm{pK}_{\mathrm{a}}$ values $\leq 10$, the palladium-catalyzed asymmetric alkylation of allylic acetates with $\mathrm{FBSM}^{[196]}$ most likely occurs via external addition of the fluoromethyl nucleophile to the chiral palladiumallyl complex to afford the enantioenriched fluoromethylated product. Cross-coupling of 2-pyridylsulfonyl iodofluoromethane with aryl iodides via copper(I) salts has been also been reported. ${ }^{[197]}$ FBSM was used in the enantioselective Mannich reaction under PTC for the synthesis of enantioenriched $\alpha$-monofluoromethylated amines. ${ }^{[198]}$ Ion-pairing between the chiral ammonium salt and the $\alpha, \alpha$-substituted fluoromethyl anion in a polar aprotic solvent gives rise to enantioselective addition to the imine intermediate.
Organocatalysts were used as bifunctional catalysts for the enantioselective addition of $\alpha$-fluoro- $\alpha$ nitro(phenylsulfonyl)methane (Scheme 43) ${ }^{[199]}$ or FBSM (Scheme $44)^{[200]}$ to $\alpha, \beta$-unsaturated ketones with cinchona alkaloid-derived catalysts 2.2 and $\mathbf{2 . 3}$ to afford $\beta$-fluoromethylated ketones. Hydrogen-bonding between both the electrophile and nucleophile with the quinuclidine moiety as well as the thiourea (Scheme 43 ) or amine (Scheme 44) is postulated to induce enantioselectivity.

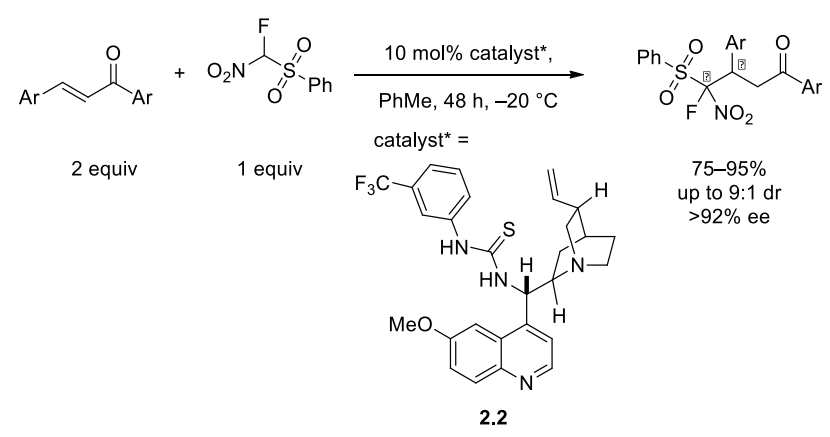

Scheme 43. Organocatalytic enantioselective 1,4-conjugate addition of fluoromethyl pronucleophile to $\alpha, \beta$-unsaturated aryl ketones

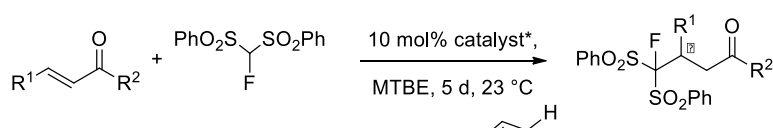

$$
\begin{aligned}
& 1 \text { equiv } 1.2 \text { equiv } \text { catalyst }^{*}={ }_{80-93 \%}^{85-93 \%} \\
& \text { (1) } \\
& 2.3
\end{aligned}
$$

Scheme 44. Organocatalytic enantioselective 1,4-conjugate addition of fluoromethyl pronucleophile to $\alpha, \beta$-unsaturated aryl ketones

\section{Difluoromethylation}

Molecules containing $\alpha$-fluoro and $\alpha, \alpha$-difluoro groups are important components of pesticides and pharmaceuticals. ${ }^{[201]}$ The $\alpha, \alpha$-difluoromethyl group has been examined for its propensity to serve as a hydrogen bond donor as potential replacement for hydroxyl groups inter alia. ${ }^{[202]}$ Many approaches towards electrophilic difluoromethylation as well as electrophilic trifluoromethylation (chapter 4), are based on radical reactivity. This is likely due to the stability imparted by multiple fluorine substitution on radical intermediates and explains the scarcity of radical reactions that achieve electrophilic monofluoromethylation. Additionally, a significant number of electrophilic difluoromethylation methods involve difluorocarbene intermediates. The $\pi$-donating ability of fluorine imparts stability difluorocarbene as well as difluoromethyl radicals compared to their nonfluorinated counterparts and leads to moderate, controlled reactivity. ${ }^{[13 \mathrm{~g}]}$ The increased acidity of $\mathrm{XF}_{2} \mathrm{C}-\mathrm{H}$ compared to $\mathrm{XFHC}-\mathrm{H}$ facilitates the formation of $\mathrm{F}_{2} \mathrm{CX}^{-}$, where $\mathrm{X}$ is a leaving group, the precursor to difluorocarbenes. In developing nucleophilic difluoromethylation reactions, the challenge of difluoromethyl anion being prone to $\alpha$ elimination and decomposition, needs to be overcome and this is often addressed through the use of electron-withdrawing groupstabilized pronucleophiles. Difluoromethyl pronucleophiles such as 
difluoromethyl phenyl sulfone typically contain one substituent that can provide mesomeric stabilization to the anion and often another substituent (usually a halide or silyl group), which is used to generate the anion.

\subsection{Electrophilic methods for difluoromethylation}

\subsubsection{Radical difluoromethylation}

Radical difluoromethylation of alkenes and alkynes has been reported with difluoroiodomethane facilitated by sodium dithionite as a radical initiator (Scheme 45). ${ }^{[203]}$ Similarly, difluorodibromomethane or difluorobromochloromethane in combination with the radical initiator $\mathrm{CuCl}$ is used for the difluoromethylation of olefins. ${ }^{[204]}$ Radical $\alpha$-difluoromethylation of enamines and ynamines ${ }^{[205]}$ can be performed with difluorodihalomethane reagents under UV irradiation. Additionally, alkenes can be difluoromethylated with halodifluorosulfides, ${ }^{[206]}$ halodifluorosulfonyl reagents, ${ }^{[207]}$ halodifluorosulfonamide reagents, ${ }^{[208]}$ halodifluorosulfanyl reagents, ${ }^{[209]}$ and halodifluoromethane. ${ }^{[203,210]}$ in the presence of appropriate radical initiators. $\alpha$-Difluoromethylation of enolates with iododifluoroacetate can be mediated by the radical initiator triethylborane (Scheme 46) ${ }^{[211]}$ After saponification of the imide, decarboxylative bromination of the resulting carboxylic acid to afford the difluorobromomethyl group followed by reduction of the carbon-bromine bond yields the difluoromethylated product. An example of enantioselective $\alpha$-difluoromethylation of octanal was reported using photoredox catalysis and ethyl difluoroiodoacetate. $^{[212]}$

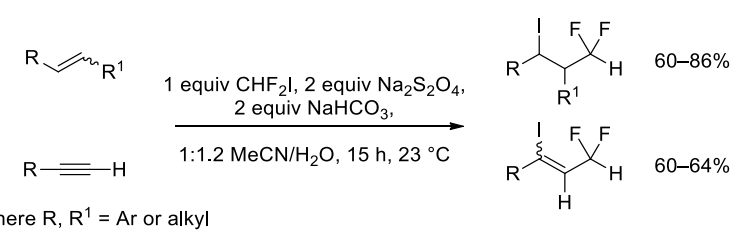

Scheme 45. Radical addition of iododifluoromethane across alkenes and alkynes

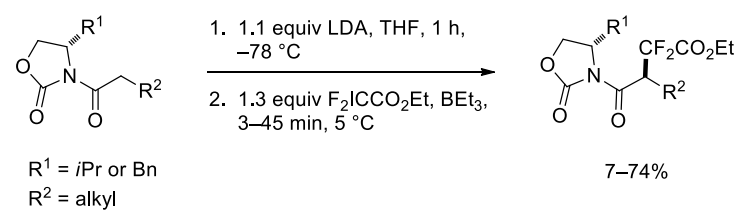

Scheme 46. Diastereoselective chiral auxiliary-mediated $\alpha$-alkylation with difluoromethylating reagent

Radical difluoromethylation of $\mathrm{C}-\mathrm{H}$ bonds in heteroarenes with tert-butylhydrogen peroxide and crystalline, bench-top stable $\mathrm{Zn}\left(\mathrm{SO}_{2} \mathrm{CF}_{2} \mathrm{H}\right)_{2}$ at ambient temperature and atmosphere was established by the Baran group (Scheme 47). ${ }^{[213]}$ Stable fluoroalkyl metal sulfinate complexes for the generation of trifluoromethyl radicals were previously used for the radical trifluoromethylation of heteroarenes, but $\mathrm{Zn}\left(\mathrm{SO}_{2} \mathrm{CF}_{2} \mathrm{H}\right)_{2}$ as a source of difluoromethyl radicals gives access to a larger variety and higher yield of functionalized heteroarenes. In the reaction, difluoromethyl radicals are generated and observed to preferentially react with the electron- poor sites of arenes and electron-deficient $\pi$-systems such as enones at the $\beta$-position, likely due to the electrophilic character of difluoromethyl radicals.
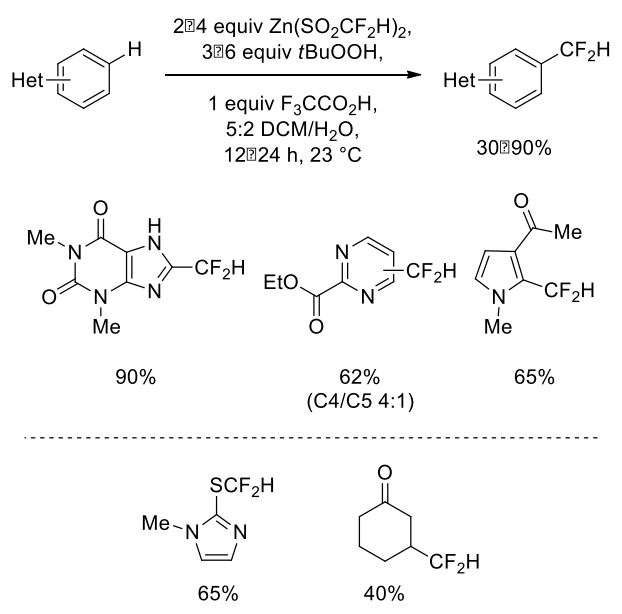

Scheme 47. Radical difluoromethylation of heteroarenes with $\mathrm{Zn}\left(\mathrm{SO}_{2} \mathrm{CF}_{2} \mathrm{H}\right)_{2}$ and tert-butylhydrogen peroxide

\subsubsection{Electrophilic difluoromethylation through carbene intermediates}

Aryl difluoromethyl ethers and thioethers can be synthesized from phenoxides ${ }^{[214]}$ and thiophenolates ${ }^{[214 \mathrm{c}-\mathrm{e}, 214 \mathrm{~g}]}$ with sources of difluorocarbene such as difluorohalomethane, ${ }^{[214 a, 214 c,}$ d] difluorohalomethane, ${ }^{[214 \mathrm{~b},} \quad{ }^{214 \mathrm{e}]} \quad$ diethylbromodifluoromethyl phosphonate (Scheme 48), ${ }^{[14 \mathrm{~g}]}$ and fluorosulfonyl difluoroacetic acid reagents. ${ }^{[214 \mathrm{f}]}$ Difluoromethylation with diethylbromodifluoromethyl phosphonate, as described by Zafrani and Segall, has proven most successful, allowing for difluoromethylation of simple electron-rich or -poor phenols and thiophenols in $60-96 \%$ yield (Scheme 48). ${ }^{[214 \mathrm{~g}]}$ In addition, enolizable functional groups such as acetyl groups do not undergo side reactions with the reagent. The proposed mechanism for the generation of difluorocarbene from diethylbromodifluoromethyl phosphonate consists in saponification of diethyl phosphonate with release of difluorobromomethyl anion followed by $\alpha$-elimination of bromide from difluorobromomethyl anion to give difluorocarbene.

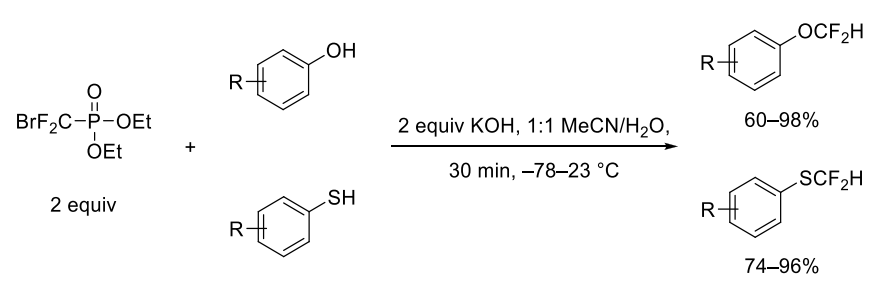

Scheme 48. Difluoromethylation of phenols and thiophenols with ethyl bromodifluoromethylphosphanate

Fluorinated alcohols, ${ }^{[214 f, 215]}$ quinoxalinones, ${ }^{[216]}$ as well as amides and oximes ${ }^{[217]}$ can undergo $O$-difluoromethylation with various difluoromethylating reagents. Non-fluorinated aliphatic alcohols remain problematic substrates for difluoromethylation, often giving rise to low yields. ${ }^{[218]}$ Likewise, nitrogenous nucleophiles such as azoles, ${ }^{[219]} 2$-mercaptoazoles, ${ }^{[220]}$ 
sulphonamides, ${ }^{[221]}$ sulfanyltetrazoles, ${ }^{[222]}$ benzotriazoles and tetrazoles, ${ }^{[223]} 2$-acetaminopyridine, ${ }^{[224]}$ and indazoles ${ }^{[225]}$ can be converted into $N$-difluoromethylated products. Historically, other reagents used in $\mathrm{N}$-difluoromethylations include $\mathrm{Zn}\left(\mathrm{CF}_{3}\right) \mathrm{Br}$, ${ }^{[226]}$ $\mathrm{Cd}\left(\mathrm{CF}_{3}\right)_{2},{ }^{[226 \mathrm{a}, 226 \mathrm{~b}, 226 \mathrm{~d}, 227]}$ and $\mathrm{Bi}\left(\mathrm{CF}_{3}\right)_{3} / \mathrm{AlCl}_{3},{ }^{[226 \mathrm{~d}]}$ all of which react through difluorocarbene intermediates. More recently, nonozone-depleting reagents 2-chloro-2,2-difluoroacetophenone ${ }^{[228]}$ and chlorodifluoromethyl phenyl sulfone (Scheme 49) ${ }^{[229]}$ have been used to generate difluorocarbene in the presence of nucleophiles to afford $O$ - and $N$-difluoromethylated products.

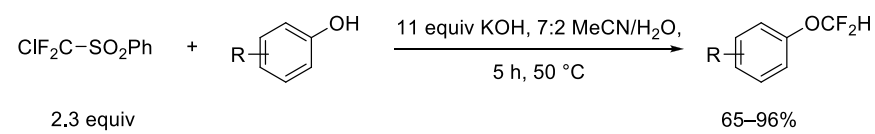

Scheme 49. Difluoromethylation of phenols with chlorodifluoromethyl phenyl sulfone

Various nucleophiles including tertiary amines, imidazoles, phosphines, and sulfonates can be treated with difluoromethylsulfonium reagent 3.1 developed by Prakash to afford $\mathrm{N}-, \mathrm{P}_{-}$, and $\mathrm{O}$ - difluoromethylated products (Scheme 50); however, the reagent did not give the corresponding difluoromethylated products with either phenols, carbon-based nucleophiles or primary/secondary amines. Decomposition of the reagent via deprotonation of the acidic proton on the difluoromethyl group in the presence of the nucleophile may provide a rationale for why some more basic nucleophiles prove incompatible with the reagent. ${ }^{[230]}$ Similarly, $\quad N, N$-dimethyl-S-difluoromethyl- $S$ phenylsulfoximinium tetrafluoroborate can be used to alkylate $N$-, $P$-, and $S$-nucleophiles. ${ }^{[218]}$

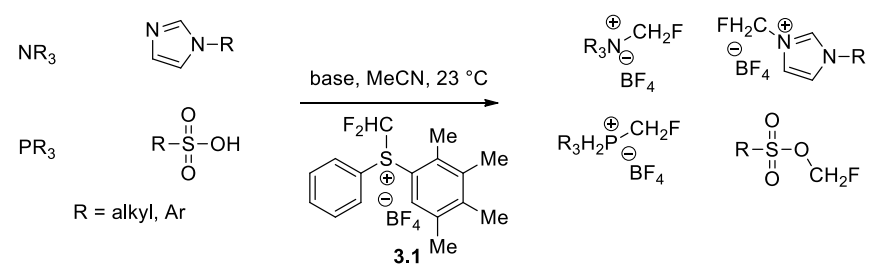

Scheme 50. Difluoroalkylation of tertiary amines and phosphines, imidazoles, and sulfonic acids

Difluoromethylation of $C$-nucleophiles with $\mathrm{pK}_{\mathrm{a}}$ values (in DMSO) in the range of 16.3 to 19.1 can be accomplished with chlorodifluoromethane (Freon R-22) and affords products in 7-64\% yield. ${ }^{[231]}$ Treatment of enolates with chlorodifluoromethane gives rise to $\alpha$-difluoromethylated carbonyl products, ${ }^{[232]}$ while diasteroselective $\alpha$-difluoromethylation of $N$-acyloxazolidinones utilizes bromodifluoromethane. ${ }^{[233]}$ Trimethylsilyl fluorosulfonyldifluoroacetate (TFDA), which also acts as a source of difluorocarbene, reacts with alkenes, ${ }^{[234]}$ alkynes, ${ }^{[234 \mathrm{~d}]}$ and ketones $^{[235]}$ to form gem-difluorocyclopropanes, gemdifluorocyclopropenes, and gem-difluoropropane ethers respectively Less commonly, this reagent is used for difluoromethylation reactions at heteroatoms. ${ }^{[234 a, 236]}$

\subsubsection{Electrophilic difluoromethylation by other mechanisms}

The development of alkylating reagent $N$-tosyl-Sdifluoromethyl-S-phenylsulfoximine led to the successful difluoromethylation of $S$-, $N$-, and $C$-nucleophiles (Scheme 51). ${ }^{[237]}$ The results of deuterium-exchange studies have rendered the formation of difluorocarbene intermediates unlikely since deprotonation/protonation steps would likely occur in the mechanism, leading to deuterium incorporation, which was not observed. This observation may provide a rationale for why the sulfoximinium reagent is not competent for the difluoromethylation of phenols, which have been shown to react with difluorocarbene. Aryl $\alpha, \alpha$-difluoroethers can be synthesized, however, via oxidative rearrangement of aldehydes with difluoroarylbromane. ${ }^{[238]}$

$$
\begin{aligned}
& \mathrm{R}_{\mathrm{SH}}^{1} \\
& \mathrm{R}^{4}=\mathrm{H} \quad \mathrm{R}^{4}=\mathrm{CF}_{2} \mathrm{H} \\
& R^{1}=a \mid k y l, A r \\
& \mathrm{R}^{2}=\mathrm{H}, \mathrm{Ar} \\
& \mathrm{R}^{3}=\mathrm{H}, \mathrm{NO}_{2}, \mathrm{Me} \\
& \mathrm{R}^{4}=\mathrm{Ar} \\
& 14 \mathrm{~h}, 60^{\circ} \mathrm{C}
\end{aligned}
$$

Scheme 51. Difluoromethylation $S$-, $C$-, $N$-nucleophiles with $N$-tosyl$S$-difluoromethyl-S-phenylsulfoximine

When iodotrifluoromethane is treated with lithium enolates, alkylation occurs selectively with fluoride displacement instead of iodide displacement. ${ }^{[239]}$ The selective fluoride displacement is attributed to activation of fluoride with lithium, driven by the formation of lithium fluoride, which has the highest alkali metalhalide lattice energy. ${ }^{[240]}$ Copper-catalyzed decarboxylative difluoromethylation of $\alpha, \beta$-unsaturated carboxylic acids was accomplished with electrophilic iodonium-based difluoromethyl reagent 3.2 in $60-91 \%$ yield with stereocontrol of double-bond geometry (Scheme 52). ${ }^{[241]}$ Difluoromethylation of boronic acids can be accomplished with copper, ethyl difluoroiodoacetate and $N, N$-diethyl-difluoroiodoacetamide. ${ }^{[242]}$

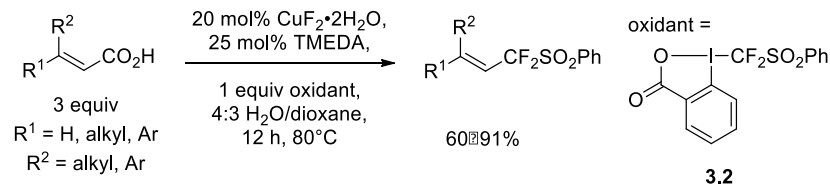

Scheme 52. Cu-catalyzed decarboxylative difluoromethylation of $\alpha, \beta-$ unsaturated carboxylic acids

\subsection{Nucleophilic methods for difluoromethylation}

Nucleophilic approaches to the synthesis of $\alpha, \alpha$ difluoromethylene ethers are still in use, but their importance declined with the development of milder, high-yielding electrophilic difluoromethylation methods. Nucleophilic fluorodesulfurization of thionoesters ${ }^{[141 \mathrm{a}, 141]}$ and $\operatorname{xanthates}^{[205,214 \mathrm{~b}, 243]}$ in addition to nucleophilic fluorodeoxygenation of aryl formylesters ${ }^{[244]}$ have been used to synthesize $\alpha, \alpha$-difluorinated ethers. Similarly the $\alpha, \alpha$ difluorination of thioacetal-protected esters can be accomplished with TREAT $-\mathrm{HF}$ and bromine. ${ }^{[245]}$ Fluorination of carbonates with $\mathrm{SF}_{4}$ results in bis(alkoxy)difluoromethane products. ${ }^{[246]}$ An extensive review was published on the synthesis of $\alpha$-fluorinated ethers, sulfides, amines, and phosphines. ${ }^{[201 b]}$ 


\subsubsection{Nucleophilic difluorination to form $\mathrm{C}_{s p 3}-\mathrm{CF}_{2} \mathrm{X}$ bonds}

C-H acidic difluoromethyl pronucleophiles containing sulfone, sulfoxide, or phosphonate electron-withdrawing groups are deprotonated prior to reaction with alkyl halides, ${ }^{[247]}$ carbonyl substrates, ${ }^{[190,248]}$ chiral sulfinylimines, ${ }^{[249]}$ amino sulfinylimines ${ }^{[188]}$ and cyclic sulfates (Scheme 53) and sulfimidates. ${ }^{[250]}$ Difluoromethyl phenyl sulfoxide ${ }^{[251]}$ and phosphonate ${ }^{[252]}$ reagents have also been used. The electron-withdrawing group in the pronucleophile used for nucleophilic difluoromethylation such as a sulfone is cleaved to give the difluoromethyl group after the pronucleophile is introduced into the substrate. The aforementioned difluoromethyl pronucleophiles are readily prepared, ${ }^{[214 \mathrm{e}, 214 \mathrm{~g}, 253]}$ thermally stable after deprotonation, ${ }^{[254]}$ and the electronwithdrawing group can be removed after difluoromethylation. ${ }^{[254]}$ Phase-transfer catalysis with $\mathbf{3 . 3}$ for the enantioselective addition of difluoromethyl phenyl sulfone to aldehydes was explored, with enantioselectivity ranging from $4-64 \%$ ee (Scheme 54$).{ }^{[255]}$

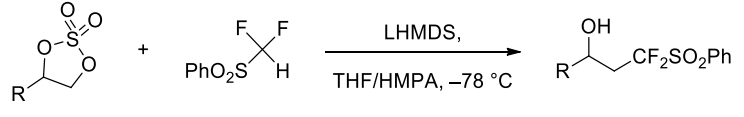

$$
\begin{aligned}
& \mathrm{R}=\mathrm{alky} \mid, \mathrm{Ar}
\end{aligned}
$$

Scheme 53. Ring opening of cyclic sulfates with difluoromethy phenyl sulfone

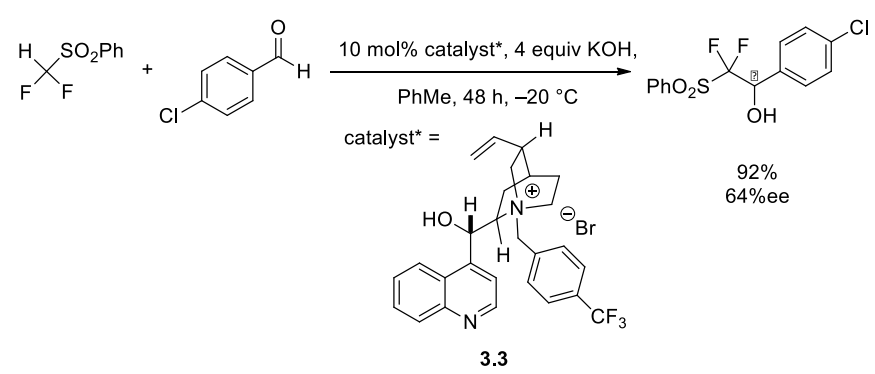

Scheme 54. Enantioselective addition of fluoromethyl pronucleophile to aryl aldehydes using a chiral ammonium cinchona alkaloid catalyst

The electron-rich organic reductant tetrakis(dimethylamino)ethylene (TDAE) has an oxidation potential similar to zinc metal ${ }^{[256]}$ and can transfer two-electrons via sequential single-electron transfer (SET). TDAE is used to reduce the carbon-halogen bond in difluoromethyl pronucleophiles to generate difluoromethyl anion equivalents which can react with aldehydes. ${ }^{[257]}$ Reaction of trimethylsilyldifluoromethyl pronucleophiles with carbonyl substrates are mediated by fluoride or Lewis acids. ${ }^{[258]}$ Advantages of utilizing silyl-based difluoromethyl pronucleophiles include easy preparation of the reagents ${ }^{[259]}$ and compatibility with enolizable carbonyl compounds. More recently, reagent $\quad(R)$ - $N$-tert-butyldimethylsilyl-S-difluoromethyl-S phenylsulfoximine has been developed for the diastereoselective nucleophilic difluoromethylation of aryl ketones. ${ }^{[260]}$

\subsubsection{Nucleophilic difluorination to form $\mathrm{C}_{s p 2}-\mathrm{CF}_{2} \mathrm{X}$ bonds}

In the presence of copper chloride, cadmium difluoromethylphosphonate reagents can be cross-coupled with iodoarenes to afford aryldifluoromethylphosphonates (Scheme 55). ${ }^{[261]}$ Similarly, copper-based difluoromethylating reagents derived from halodifluoroacetate undergo cross-coupling with aryl or alkenyl iodides. ${ }^{[262]}$ The copper-catalyzed cross-coupling of aryl halides and ethyl trimethylsilyldifluoroacetate to yield substituted difluoromethyl benzoates, which, after saponification and decarboxylation, afford difluoromethylarenes, was reported by Amii. [263] Ethyl ortho-iodobenzoate derivatives can undergo coppercatalyzed cross-coupling with zinc diethyldifluoromethylphosphonate as reported by Zhang. ${ }^{[264]}$ Direct difluoromethylation of aryl and alkenyl iodides using five equivalents of $\mathrm{TMSCF}_{2} \mathrm{H}$ as the difluoromethyl pronucleophile with copper salts was established by Hartwig and co-workers (Scheme 56). ${ }^{[265]}$ The latter method tolerates electron-rich as well as electronpoor functional groups and directly affords the desired difluoromethylated products in $30-91 \%$ isolated yield. Prakash and co-workers recently developed a method for the difluoromethylation of aryl and alkenyl halides as well as heteroaryl halides with $\mathrm{Bu}_{3} \mathrm{SnCF}_{2} \mathrm{H}$ and copper salts (Scheme 57). ${ }^{[266]}$ Difluoromethyl arenes can also be synthesized by nucleophilic deoxyfluorination of aromatic aldehydes with Deoxo-Fluor ${ }^{\circledR}$. ${ }^{[267]}$

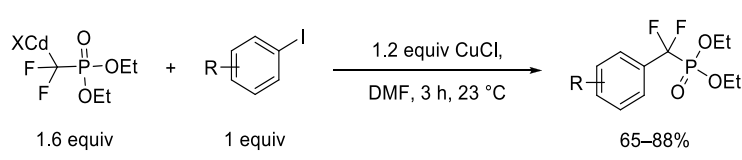

Scheme 55. Cu-mediated cross-coupling of cadmium-based difluoromethyl pronucleophile with aryl iodides

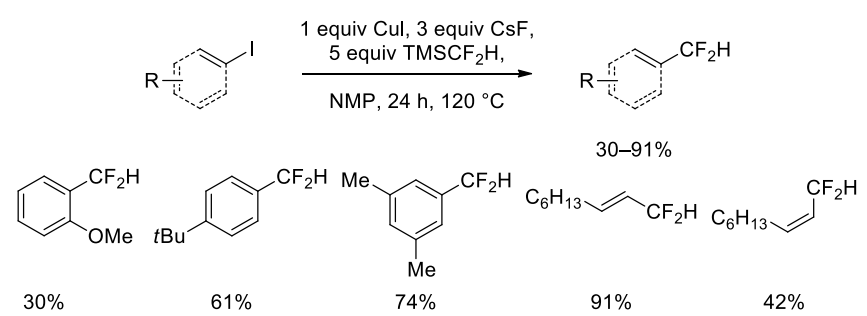

Scheme 56. Direct difluoromethylation of aryl and alkenyl iodides with copper iodide and $\mathrm{TMSCF}_{2} \mathrm{H}$

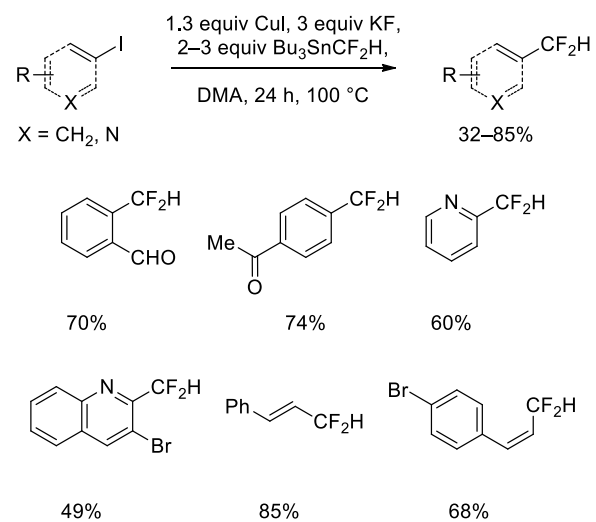

Scheme 57. Direct difluoromethylation of aryl, heteroaryl, and alkenyl iodides with copper iodide and $\mathrm{Bu}_{3} \mathrm{SnCF}_{2} \mathrm{H}$

\section{Trifluoromethylation}

Trifluoromethyl groups are electron-withdrawing substituents that increase the lipophilicity of molecules. ${ }^{[15]}$ Functionalization of 
anti-cancer agent epothilone with a trifluoromethyl group (later named fludelone) serves as an example of how trifluoromethylation can increase the metabolic stability of the molecule while retaining comparable cytotoxic potency. ${ }^{[268]}$ Hence, there is considerable interest in developing methods for controlled introduction of trifluoromethyl groups into small molecules. Compared to monoand difluoromethylation, fewer synthesis strategies are available for the introduction of trifluoromethyl groups; because three of the four substituents on the carbon atom are pre-determined, only one other substituent can be varied, which limits potential synthetic handles. Transition-metal-mediated trifluoromethylation is complicated by the strong metal- $\mathrm{CF}_{3}$ bond originating from both the polar contribution of the bond as well as backbonding from filled metal d orbitals into the $\sigma^{*}{ }_{\mathrm{C}-\mathrm{F}}$ bonds, which therefore results in a high barrier to $\mathrm{C}-\mathrm{CF}_{3}$ bond formation.

\subsection{Electrophilic trifluoromethylation}

A range of substrates can be trifluoromethylated employing functional group tolerant reagents and conditions. In laboratory settings, crystalline and easily weighable electrophilic trifluoromethylating reagents are preferable due to ease of handling, while for industrial processes lower cost reagents such as $\mathrm{CF}_{3} \mathrm{I}$, or even better $\mathrm{CF}_{3} \mathrm{H}$, are favored.

The development of widely used crystalline electrophilic trifluoromethylating reagents (Figure 3$)^{[269]}$ such as the Togni reagents, ${ }^{[270]} S$-(trifluoromethyl)dibenzothiophenium salts, ${ }^{[271]}$ and $S$-trifluoromethyldiarylsulfonium salts ${ }^{[272]}$ has enabled the development of functional group tolerant methods that encompass a broad substrate scope. These trifluoromethylating reagents are twoelectron oxidants but the exact mechanisms for trifluoromethylation have not been firmly established in many cases; the mechanism could occur via two SET or one two-electron transfer concurrent with or followed by trifluoromethyl group transfer. ${ }^{[273]}$
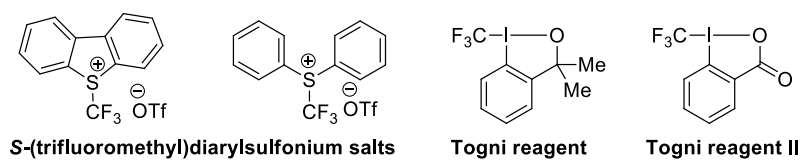

Figure 3. A selection of widely used electrophilic trifluoromethylating reagents

An alternative to using electrophilic trifluoromethylating reagents is the use of nucleophilic trifluoromethylating reagents in conjunction with oxidants to accomplish oxidative trifluoromethylation. Transition-metals have facilitated the aryl carbon-trifluoromethyl group bond formation in trifluoromethyl arenes from aryl nucleophiles by exploiting redox chemistry at the metal center. Organocatalysts, on the other hand, have allowed for the development of enantioselective $\alpha$-trifluoromethylation reactions ${ }^{[274]}$ of carbonyl substrates via radical and non-radical mechanisms.

\subsubsection{Electrophilic methods for the synthesis of trifluoromethylated $s p^{2}$ carbon centers via radicals}

Initial forays into radical trifluoromethylation of arenes utilized photochemically-induced radical addition of trifluoroiodomethane to substituted benzenes ${ }^{[275]}$ and imidazoles ${ }^{[276]}$ as well as flash thermolysis of aryl trifluoroacetates ${ }^{[277]}$ to afford a mixture of trifluoromethylated products in circa $15 \%$ yield. Copper(II)catalyzed electrophilic radical trifluoromethylation was later established using sodium trifluoromethylsulfinate for trifluoromethylation of electron-rich arenes. ${ }^{[278]}$ Ensuing endeavors have focused on developing trifluoromethylation methods that afford trifluoromethylated product in high yield and selectivity, which was recently accomplished via radical trifluoromethylation. ${ }^{[279]}$

Direct trifluoromethylation of $\mathrm{C}-\mathrm{H}$ bonds would obviate the need for pre-functionalization, but is rendered difficult by the inertness of arene $\mathrm{C}-\mathrm{H}$ bonds. In the case of $\mathrm{N}$-heteroarenes and electron-rich arenes, reaction with Togni's reagent affords multiple trifluoromethylated products for most substrates. ${ }^{[280]}$ The use of solid and bench-top stable reagent $\mathrm{NaSO}_{2} \mathrm{CF}_{3}$ in radical trifluoromethylations was first described by Langlois for the trifluoromethylation of electron-rich arenes. ${ }^{[278]}$ Baran and coworkers used the Langlois reagent in conjunction with excess tertbutyl hydrogen peroxide for the trifluoromethylation of heteroarenes (Scheme 58). ${ }^{[281]}$ The method is operationally simple, takes place at ambient temperature and atmosphere through in situ generation of electrophilic $\mathrm{CF}_{3}$ radicals without additional catalysts. The selectivity for this reaction ranges from a $1: 1$ isomeric product mixture to the formation of a single isomer. The Baran group has since improved radical trifluoromethylation with $\mathrm{Zn}\left(\mathrm{SO}_{2} \mathrm{CF}_{3}\right)_{2}$ and extended the use of other fluorinated zinc sulfinate salts to include a variety of fluorinated derivatives. ${ }^{[213 b]}$ Photoredox catalysis with a redox-active ruthenium(II) catalyst and $\mathrm{CF}_{3} \mathrm{SO}_{2} \mathrm{Cl}$, as described by MacMillan and co-workers, has been used to generate the electrophilic $\mathrm{CF}_{3}$ radical and leads to the formation of monotrifluoromethylated products for heteroarenes and trifluoromethylated arenes (Scheme 59). ${ }^{[282]}$ The trifluoromethylation methods decribed by Baran and MacMillan allow for the efficient direct synthesis of complex trifluoromethyl arenes, which had not previously been possible. Iron-catalyzed radical trifluoromethylation of arenes and heteroarenes yields less than $50 \%$ for most substrates, ${ }^{[283]}$ while $\mathrm{Fe}$ (II)-catalyzed trifluoromethylation of potassium alkenyltrifluoroborates with Tognis reagent II affords the desired trifluoromethylated alkenes in $49-79 \%$ yield in up to $>95: 5 \mathrm{E} / \mathrm{Z}$ selectivity (Scheme 60). ${ }^{[284]}$ Silver-mediated trifluoromethylation of unactivated arenes and heteroarenes with $\mathrm{TMSCF}_{3}$ is likewise postulated to occur via a radical mechanism to afford trifluoromethylated products (Scheme 61). ${ }^{[285]}$ 

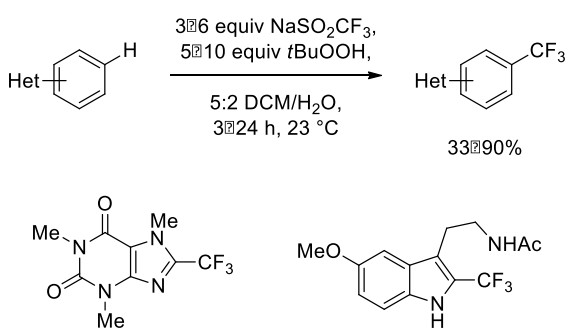

$84 \%$

(from caffeine)

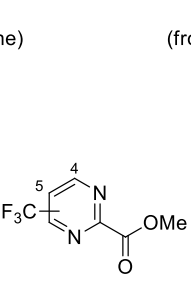

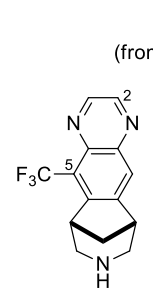

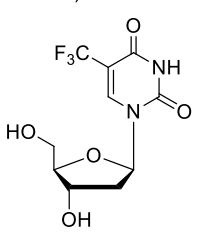

$57 \%$

$37 \%$
$(\mathrm{C} 4 / \mathrm{C} 5$ 1:1)

(rom Chantix)

(trifluridine)

.

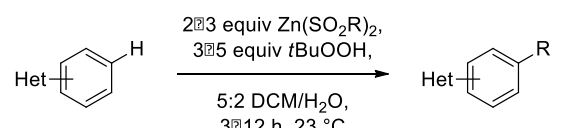

$$
21-89 \%
$$

where $\mathrm{R}=\mathrm{CF}_{3}, \mathrm{CF}_{2} \mathrm{H}$

$\mathrm{CH}_{2} \mathrm{CF}_{3}$, or $\mathrm{CH}_{2} \mathrm{~F}$

Me

$\overbrace{3}^{N} \overbrace{0}^{6} \mathrm{OEt}$

$\mathrm{R}=\mathrm{CF}_{3}, 89 \% \quad \mathrm{R}=\mathrm{CF}_{3}, 35 \%(\mathrm{C} 2 / \mathrm{C} 34: 1)$

$\mathrm{CF}_{2} \mathrm{H}, 73 \% \quad \mathrm{CF}_{2} \mathrm{H}, 66 \%$ (C2 only)

$\mathrm{CH}_{2} \mathrm{CF}_{3}, 51 \% \quad \mathrm{CH}_{2} \mathrm{CF}_{3}, 18 \%(\mathrm{C} 2 / \mathrm{C} 34: 1)$

$\mathrm{CH}_{2} \mathrm{~F}, 80 \%$

$\mathrm{CH}_{2} \mathrm{~F}, 73 \%(\mathrm{C} 2 /[\mathrm{C} 2+\mathrm{C} 6]$ 17:1)

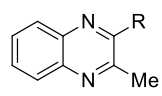

$\mathrm{R}=\mathrm{CF}_{3}, 75 \%$ (5 products)

$\mathrm{CF}_{2} \mathrm{H}, 50 \%$

$\mathrm{CH}_{2} \mathrm{CF}_{3}, 31 \%$

$\mathrm{CH}_{2} \mathrm{~F}, 56 \%$

Scheme 58. Radical trifluoromethylation of heteroarenes

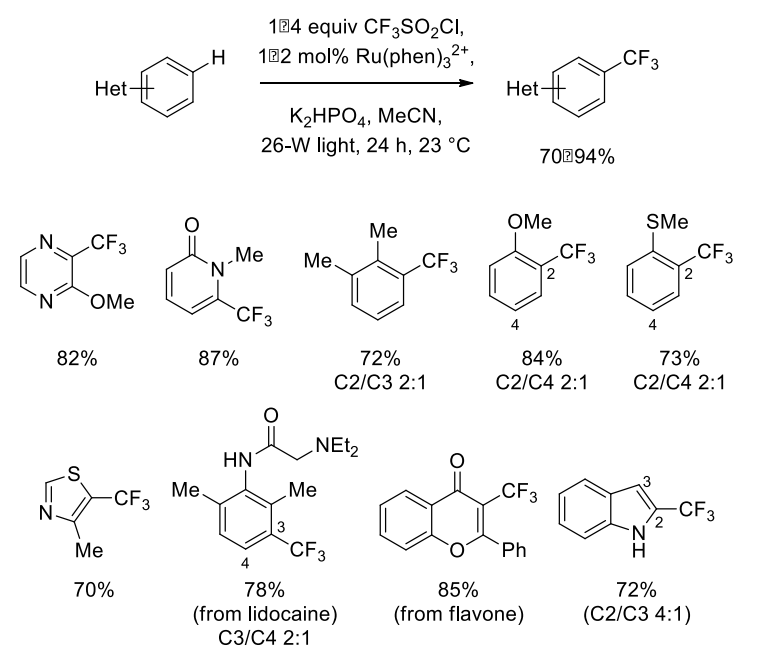

Scheme 59. Ru-photoredox-catalyzed trifluoromethylation of arenes and heteroarenes

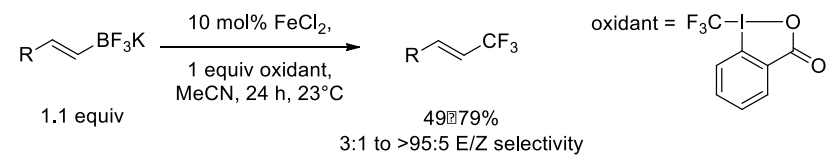

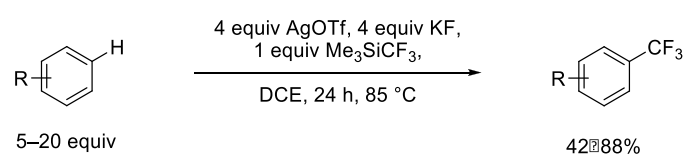

Scheme 61. Ag-mediated trifluoromethylation of unactivated arenes

\subsubsection{Electrophilic methods for the synthesis of trifluoromethylated $s p$ and $s p^{2}$ carbon centers with copper}

The trifluoromethylation of boronic acids has been successfully accomplished with copper catalysts via two approaches: 1) oxidation with an electrophilic trifluoromethylating reagent or 2) using an oxidant in conjunction with trifluoromethyl anion equivalent. Trifluoromethylation of aryl and alkenylboronic acids with electrophilic trifluoromethylating reagents was reported with Togni reagent as described by Shen (Scheme 62) ${ }^{[286]}$ and diaryltrifluoromethylsulfonium salts as described by $\mathrm{Liu}^{[287]}$ and Xiao. ${ }^{[288]}$ Shen's method encompasses a broad range of boronic acid substrates and affords trifluoromethylated products in 50-95\% yield while utilizing a lower copper catalyst loading. The use of the combination between a nucleophilic trifluormethyl source and an oxidant is illustrated by the oxidative copper-mediated trifluoromethylation of arylboronic acids and alkenylboronic acids with silver carbonate and trimethylsilyltrifluoromethane $\left(\mathrm{TMSCF}_{3}\right)$ developed by the Qing group (Scheme 63 $3^{[289]}$ ). ${ }^{[289-290]}$ Similarly, oxygen can be used as the terminal oxidant instead of less-desirable metal-based oxidants ${ }^{[291]}$ as shown by Buchwald and provides a complementary method to the one described by Shen. In lieu of $\mathrm{TMSCF}_{3}$, the crystalline and shelf-stable potassium trifluoromethyltrimethoxyborate was used by Gooßen with oxygen as a terminal oxidant. ${ }^{[292]}$ The Grushin group developed a method that utilizes low-cost fluoroform as the $\mathrm{CF}_{3}$ source in conjunction with copper complexes to trifluoromethylate arylboronic acids (Scheme 64). ${ }^{[293]}$ Sanford and co-workers demonstrated radical trifluoromethylation of arylboronic acids with $\mathrm{CF}_{3} \mathrm{I}$ and $\mathrm{Cu} / \mathrm{Ru}$ cocatalysts (Scheme 65), ${ }^{[294]}$ which subsequently was made more practical with the use of $\mathrm{NaSO}_{2} \mathrm{CF}_{3}$ as the trifluoromethyl radical source. ${ }^{[295]}$

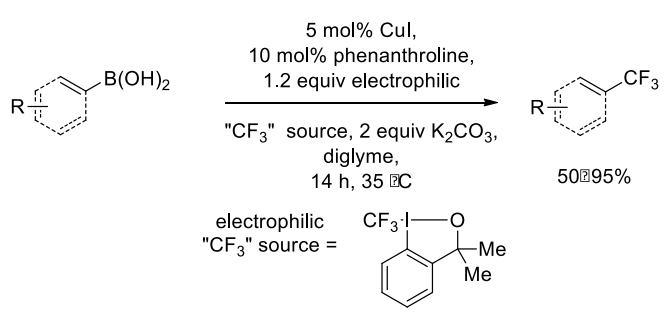

Scheme 62. Cu-catalyzed electrophilic trifluoromethylation of aryl and alkenyl boronic acids with Togni reagent

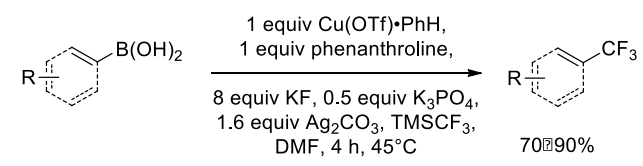

Scheme 63. Cu-mediated electrophilic trifluoromethylation of aryl and alkenyl boronic acids

Scheme 60. Fe(II)-catalyzed trifluoromethylation of potassium alkenyltrifluoroborates with Togni reagent II 


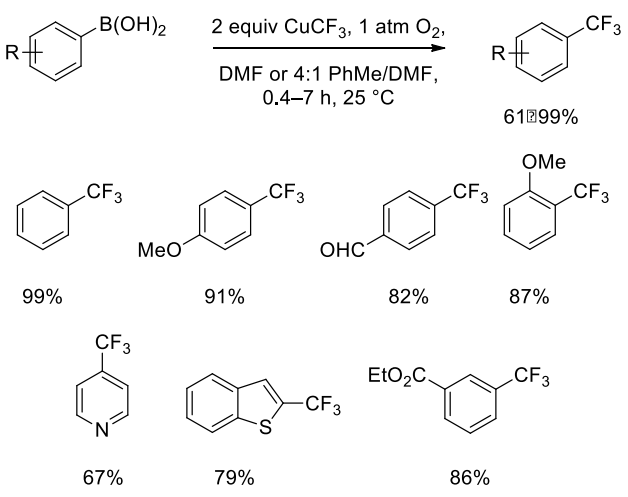

Scheme 64. Cu-mediated trifluoromethylation of aryl boronic acids with $\mathrm{CuCF}_{3}$ generated from $\mathrm{CHF}_{3}$

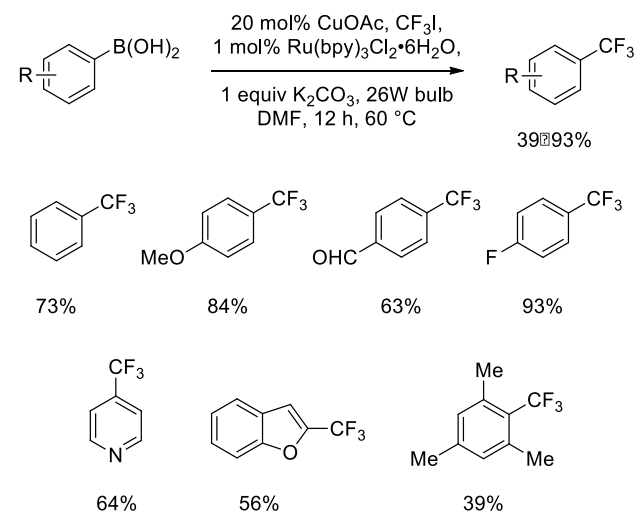

Scheme 65. Cu-catalyzed trifluoromethylation of aryl boronic acids with $\mathrm{Ru}(\mathrm{bpy})_{3} \mathrm{Cl}_{2} \cdot 6 \mathrm{H}_{2} \mathrm{O}$ and $\mathrm{CF}_{3} \mathrm{I}$

$\alpha, \beta$-Unsaturated carboxylic acids ${ }^{[241]}$ undergo copper-catalyzed decarboxylative trifluoromethylation with Togni reagent II to afford the trifluorofluoromethylated alkene products in $42-74 \%$ yield with $\geq 92: 8$ selectivity for the $E$-isomer (Scheme 66 ). This transformation reported by $\mathrm{Hu}$ allows for facile conversion of $\alpha, \beta$-unsaturated esters and other carboxylic acid derivatives to the trifluoromethylalkenes after saponification

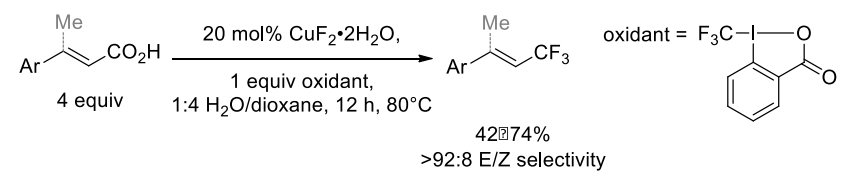

Scheme 66. Cu-catalyzed trifluoromethylation of $\alpha, \beta$-unsaturated carboxylic acids with Togni reagent II

An illustrative example of how transition metals can be employed to control reactivity is the one-pot arene $\mathrm{C}-\mathrm{H}$ borylation/trifluoromethylation sequence. $^{[296]}$ Direct trifluoromethylation of heteroarenes can be accomplished by copper-catalyzed oxidative trifluoromethylation with $\mathrm{TMSCF}_{3}$ and bis(tert-butyl)peroxide ${ }^{[297]}$ as well as with oxygen as the oxidant (Scheme 67) ${ }^{[297]}$ Oxidative trifluoromethylation of terminal alkynes

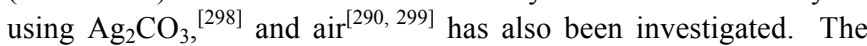
use of oxygen as the stoichiometric (terminal) oxidant avoids the creation of large amounts of waste and renders the transformations more practical and economical for large-scale trifluoromethylation reactions. Regioselective indole trifluoromethylation had been a long-standing challenge; selectivity for the $\mathrm{C} 2$ position could be accomplished via copper catalysis ${ }^{[300]}$ utilizing an electrophilic trifluoromethylating reagent and in a palladium-catalyzed ${ }^{[301]}$ transformation using $\mathrm{PhI}(\mathrm{OAc})_{2}$ and trifluoromethyl anion.

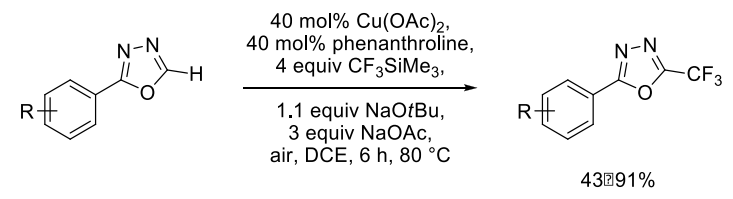

Scheme 67. Cu-catalyzed trifluoromethylation of 1,3,4-oxadiazoles

\subsubsection{Electrophilic methods for the synthesis of trifluoromethylated $s p^{2}$ carbon centers with other transition metals}

Trifluoromethylation of arenes under rhenium catalysis is possible but affords multiple isomeric products with a total yield of $11-77 \%$. ${ }^{[302]}$ Progress towards the regioselective trifluoromethylation of arenes has been made with nitrogen directing groups such as the $N$-directed electrophilic trifluoromethylation of arenes with a palladium catalyst (Scheme 68). ${ }^{[303]}$ Unlike with $N$ directed arene fluorination catalyzed by palladium, $N$-directed electrophilic trifluoromethylation of arenes do not result in ortho,ortho-ditrifluoromethylated products, most likely due to steric constraints preventing a second cyclometallation event. Similarly, by means of a triazene directing group, ortho-trifluoromethylation of aryl triazenes with $\mathrm{TMSCF}_{3}$ can be accomplished with four equivalents of silver fluoride. ${ }^{[304]}$

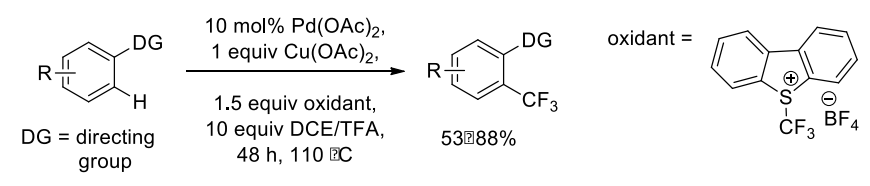

Scheme 68. Pd-catalyzed $\mathrm{N}$-directed trifluoromethylation of arenes

Reductive elimination from $\mathrm{Pd}(\mathrm{IV})$ complexes to form trifluoromethyl arenes was investigated ${ }^{[305]}$ and these studies established the viability of aryl carbon- $\mathrm{CF}_{3}$ reductive elimination from high-valent organometallic complexes (Scheme 69). ${ }^{[306]}$ Additionally, it was found that oxidation of benzoquinolinecyclometalated $\mathrm{Pd}(\mathrm{II})$ acetate dimers afforded $\mathrm{Pd}(\mathrm{III})-\mathrm{Pd}(\mathrm{III})$ dimers ${ }^{[307]}$ which, in the presence of acetic acid/water, disproportionated to $\mathrm{Pd}(\mathrm{IV})$ complexes. $^{[308]}$ These reports established that aryl trifluoromethylation could be accomplished through high-valent palladium intermediates. 


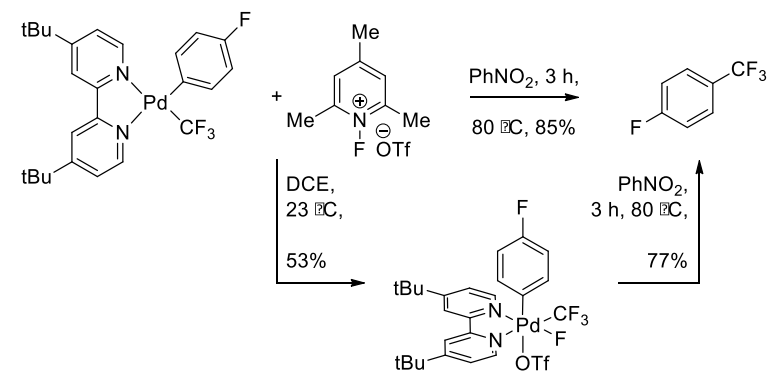

Scheme 69. Reductive elimination of Pd(IV) complexes to afford trifluoromethyl arenes

\subsubsection{Electrophilic methods for the synthesis of trifluoromethylated $s p^{3}$ carbon centers}

In 1949, Haszeldine reported the photochemical and thermal addition of trifluoromethyl radical to alkenes. ${ }^{[309]}$ The use of ruthenium(II) complexes afforded the addition products of trifluoromethylsulfonyl chloride across alkenes with extrusion of $\mathrm{SO}_{2}$ in a controlled fashion. ${ }^{[310]}$ Continued interest in controlled addition of fluorinated reagents across double bonds led to the development of lauroyl peroxide-initiated radical addition of aminotrifluoroethyldithiocarbamate across alkenes to afford $\alpha$ trifluoromethylamines. ${ }^{[311]}$ Incorporation of trifluoromethyl groups vicinal to amine functional groups has been accomplished with other approaches besides radical addition of $\alpha$-trifluoromethylated amino derivatives. These methods include benzoyl peroxide-promoted oxidative coupling between the $\alpha$-carbon of the amine and $\mathrm{TMSCF}_{3}{ }^{[312]}$ and the synthesis of anti- $\alpha$-trifluoromethyl- $\beta$-amino alcohols via three-component condensation reaction. ${ }^{[313]}$ Controlled radical trifluoromethyl-aminoalkoxylation across unactivated alkenes with Togni reagent II, TEMPO, and sodium metal has recently been reported by Studer. ${ }^{[314]}$

Chiral auxiliary-functionalized lithium imide enolates can undergo trifluoromethylation with trifluoroiodomethane in the presence of radical initiator triethylborane. ${ }^{[315]}$ This transformation was later applied more broadly to the lithium and titanium-based enolates of ketones. ${ }^{[316]}$ Similarly, chiral auxiliary-functionalized zirconium enolates can be trifluoromethylated and perfluoroalkylated in the presence of a ruthenium catalyst in up to $99 \%$ yield and $\geq 9: 1$ diastereoselectivity. ${ }^{[317]}$ Diastereoselective $\alpha$ trifluoromethylation of chiral imides was developed with Togni reagent, circumventing the need to condense iodotrifluoromethane gas for use in reactions. ${ }^{[318]}$ Cyclic ketones and carboxylic acid derivatives have also been $\alpha$-trifluoromethylated through photochemical radical processes (Scheme 70). ${ }^{[319]}$ Trifluoroacetadehyde methyl hemiacetal has been established as a source of trifluoromethyl groups through acid-mediated FriedelCrafts reaction with arenes. ${ }^{[320]}$

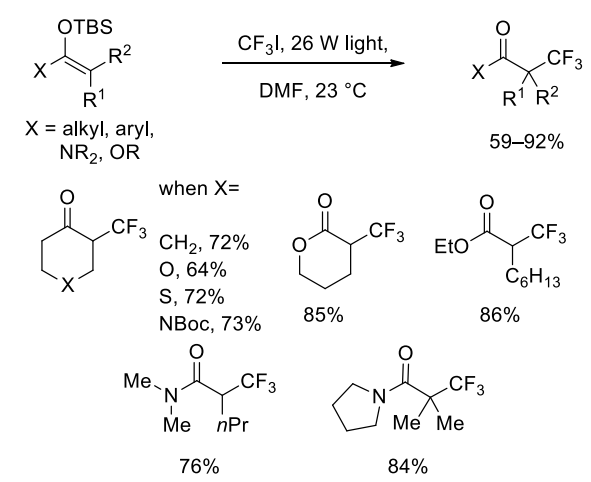

Scheme 70. Photochemical a-trifluoromethylation of silyl enol ethers

A preliminary report for enantioselective $\alpha$-trifluoromethylation of enolates by Umemoto utilizes chiral borane Lewis acid-enolate adducts and trifluoromethylsulfonium reagents to afford $\alpha$ trifluoromethyl carbonyl products. ${ }^{[271 \mathrm{c}]}$ Enantioselective trifluoromethylations of $\beta$-keto esters have been investigated with chiral guanidine bases and are postulated to occur via chiral enolateguanidinium complexes. ${ }^{[321]}$ Subsequent approaches for the enantioselective $\alpha$-trifluoromethylation of carbonyl substrates made use of organocatalysis to generate chiral enamines. Through iridium photoredox co-catalysis described by MacMillan, aldehydes were enantioselectively $\alpha$-trifluoromethylated (Scheme 71). ${ }^{[212]}$ The photoredox transformation occurs via a radical mechanism, which is likely not the case for organocatalytic $\alpha$-trifluoromethylation of aldehydes with trifluoromethyliodonium salts reported in the following year by the same group (Scheme 72). ${ }^{[322]}$ The latter method requires two equivalents of aldehyde, is operationally practical in a laboratory setting and affords products in similar yields and enantiomeric excess as the photochemical method with $\mathrm{CF}_{3} \mathrm{I}$. A mild transition-metal-catalyzed method for the $\alpha$ trifluoromethylation of $\alpha$-acidic $\beta$-ketoesters has been reported by Gade with copper catalysis in $80-99 \%$ ee (Scheme 73).

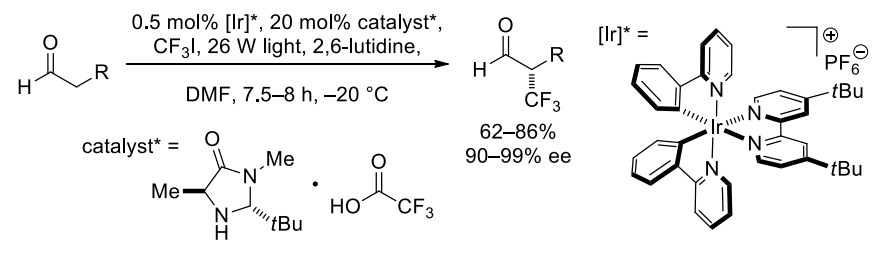

Scheme 71. Ir- photoredox-catalyzed $\alpha$-trifluoromethylation of aldehydes

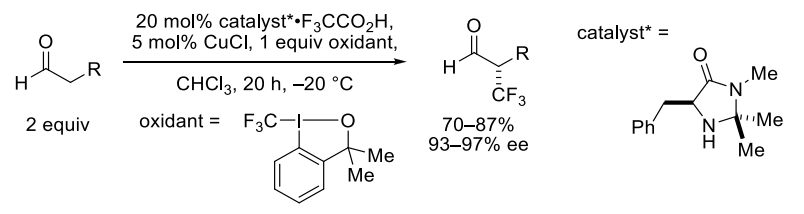

Scheme 72. Organocatalytic $\alpha$-trifluoromethylation of aldehydes with Togni reagent 


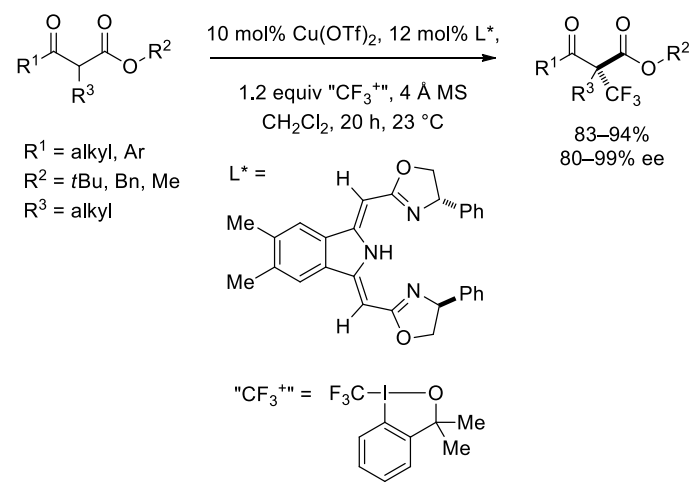

Scheme 73. Electrophilic $\alpha$-trifluoromethylation of $\beta$-ketoesters catalyzed by $\mathrm{Cu}(\mathrm{II})$ with Togni reagent I

The initial approach used for the synthesis for 3,3,3trifluoropropene groups involved trifluoromethylation of prefunctionalized allyl nucleophiles such as allylic silanes ${ }^{[323]}$ and allylic stannanes ${ }^{[324]}$ in addition to trifluoroethylation of alkenylstannanes $^{[324]}$ in addition to trifluoroethylation of alkenylstannanes. ${ }^{[324]}$ The combination of allyl halides ${ }^{[325]}$ with $\mathrm{TMSCF}_{3}$ and stoichiometric $\mathrm{Cu}$ for allylic trifluoromethylation has been reported as well. Subsequently, in an effort to obviate the need for toxic stannyl precursors, unactivated alkenes were examined as substrates for trifluoromethylation. As reported by Buchwald, terminal alkenes can undergo $\mathrm{C}-\mathrm{H}$ activation at the allylic position and oxidation with Togni reagent II in the presence of catalytic amounts of $\mathrm{Cu}(\mathrm{I})$ salts to afford $>9: 1 \mathrm{E} / \mathrm{Z}$ selectivity of terminal trifluoromethyl allyl products (Scheme 74 , top) ${ }^{[326]}$ Subsequently, this transformation was extended to the intramolecular oxytrifluoromethylation of terminal alkenes. ${ }^{[327]}$ Copper-catalyzed allylic $\mathrm{C}-\mathrm{H}$ trifluoromethylation of terminal alkenes was reported by Qing, ${ }^{[328]} \mathrm{Fu}$ and Liu, ${ }^{[329]}$ as well as Wang (Scheme 74 , bottom). ${ }^{[330]}$ The latter transformation is hypothesized by Wang and co-workers to occur via SET; Buchwald and co-workers have found through the use of radical scavengers and radical clock substrates that a mechanism involving a free allylic radical is unlikely. Additional reactivity of alkenes with electrophilic trifluoromethylating reagents include the oxidative trifluoromethylation of styrenes to give $\alpha$ trifluoromethyl ketones ${ }^{[331]}$ and an arene cyclizationtrifluoromethylation cascade with activated alkenes. ${ }^{[332]}$ More recently, oxidative trifluoromethylation of primary and secondary (Scheme 75) alkylboronic acids has been accomplished with a substoichiometic amount of copper salts in the presence of silver salts and $\mathrm{TMSCF}_{3}$ to afford the corresponding products in $36-78 \%$ yield and $35-54 \%$ yield respectively. ${ }^{[333]}$

$$
\text { equiv }
$$

Scheme 74. Cu-catalyzed allylic C-H trifluoromethylation

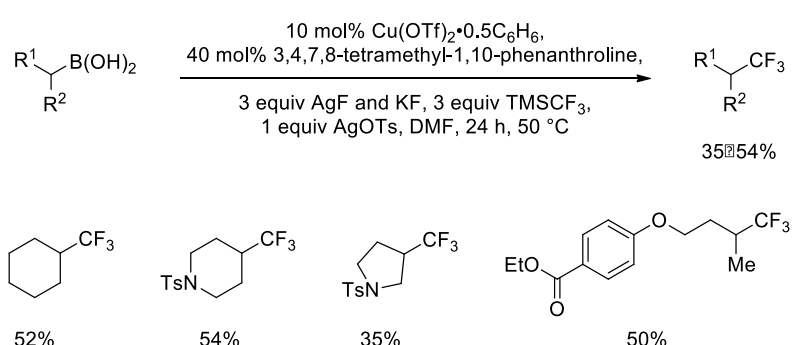

Scheme 75. Cu-promoted oxidative trifluoromethylation of secondary alkylboronic acids

Various electrophilic trifluoromethylating reagents such as trifluoromethylazosulfonylarenes ${ }^{[334]}$, perfluoroiodonium salts, ${ }^{[335]}$ (trifluoromethyl)dibenzothiophenium, selenophenium, and tellurophenium salts, ${ }^{[271 \mathrm{a},}{ }^{271 \mathrm{~b}},{ }^{336]} \quad \mathrm{N}$-trifluoromethyl $-N$ nitrosobenzenesulfonamide ${ }^{[337]}$ and $N$-trifluoromethyl- $N$-nitrosotype reagents ${ }^{[338]}$ have been synthesized for the trifluoromethylation of sulfur and carbon nucleophiles. More commonly used hypervalent iodine reagents developed by Togni have been applied to the trifluoromethylation of carbon and sulfur-based nucleophiles ${ }^{[270,273 a, 339]}$ as well as sulfonates. ${ }^{[340]}$ Additionally, fluorinated Johnson reagent [(oxido)phenyl(trifluoromethyl)- $\lambda^{4}$ sulfanylidene]dimethylammonium tetrafluoroborate has been developed as a reagent for the trifluormethylation of $\mathrm{C}-\mathrm{H}$ acidic substrates. $^{[341]}$

\subsection{Nucleophilic trifluoromethylation}

The synthesis of trifluoromethylated products was initially accomplished via nucleophilic fluoride displacement of trihalomethyl substrates or fluorodeoxygenation of carboxylic acid derivatives under harsh conditions. ${ }^{[1 \mathrm{c}, 342]}$ Subsequent approaches for nucleophilic trifluoromethylation utilized stabilized trifluoromethyl anion equivalents for cross-coupling, substitution, or addition reactions with electrophiles. Nucleophilic trifluoromethylation with trifluoromethyl anion is challenging due to a competing fluoride elimination pathway that gives rise to difluorocarbene. The relative rates of the productive and the decomposition pathway as well as the rate at which trifluormethyl anion is generated from the pronucleophile is of great importance, which renders the selection of the correct pronucleophile crucial to the success of nucleophilic trifluoromethylation methods. The trifluoromethyl anion can be generated by reduction of trifluoromethyl iodide or bromide with transition metal or organic reductants or by transmetallations of the pronucleophile to yield a metal-bound $\mathrm{CF}_{3}$ group.

$\mathrm{TMSCF}_{3}$, a commonly employed pronucleophile of trifluoromethyl anion, is a hydrolyzable, moisture sensitive liquid which, when compared to weighable, crystalline reagents, is more difficult to handle. Crystalline, weighable reagents have been developed as trifluoromethyl pronucleophiles but can be rather expensive. Trifluoroacetate salts are cheap and easy to handle, but so far no mild, functional group tolerant trifluoromethylation method has been reported using this pronucleophile. Inexpensive, gaseous trifluoromethane can be used for trifluoromethylation reactions. ${ }^{[293,343]}$

\subsubsection{Nucleophilic methods for the synthesis of trifluoromethylated $s p^{2}$ carbon centers}


Swarts first reported the synthesis of trifluoromethylbenzene in 1898 via nucleophilic fluoride substitution of the corresponding trichloride. ${ }^{[1 \mathrm{c}, 344]}$ Benzoic acid substrates were likewise exhaustively fluorinated with $\mathrm{SF}_{4}$ at the carbonyl carbon to afford the trifluoromethylated arenes. ${ }^{[342]}$ The yield for nucleophilic trifluoromethylation could be improved when copper powder and $\mathrm{CF}_{3} \mathrm{I}$ were used to synthesize trifluoromethylbenzene from iodobenzene $\mathrm{e}^{[345]}$ and subsequent optimization with different sources of copper and metal mixtures allow for lower reaction temperatures. ${ }^{[346]}$ Studies by Burton revealed that the trifluoromethyl-copper complex generated upon reduction of dihalodifluoromethane with zinc metal and metathesis with a copper(I) salt was the active species responsible for trifluoromethylation. ${ }^{[347]}$ This discovery led to the development of different methods using copper-trifluoromethyl complexes to trifluoromethylate aryl bromides, ${ }^{[348]}$ aryl chlorides, ${ }^{[349]}$ aliphatic halides, ${ }^{[350]}$ and heteroaromatic halides. ${ }^{[351]}$

Copper has been used stoichiometrically and also as a catalyst with various reagents: dibromodifluoromethane, ${ }^{[352]}$ sodium trifluoroacetate, ${ }^{[353]}$ methyl trifluoroacetate, ${ }^{[354]}$ fluorosulphonyldifluoromethyl iodide, ${ }^{[355]}$ ethyl chlorodifluoroacetate/KF, ${ }^{[356]}$ bis(trifluoromethyl)mercury, ${ }^{[357]}$ and $S$-(trifluoromethyl)diphenylsulfonium triflate. ${ }^{[358]}$ The use of $S$ (trifluoromethyl)diphenylsulfonium triflate is interesting because an electrophilic crystalline trifluoromethylating reagent can be reduced in situ to a nucleophilic trifluoromethyl-copper complex to afford trifluoromethylated heteroarenes from the corresponding iodides. Electrochemical reduction methods with a copper anode and $\mathrm{CF}_{3} \mathrm{Br}$ ${ }^{[359]}$ in addition to trifluoromethyl copper complex generation via cupration of fluoroform ${ }^{[360]}$ have both been explored for the trifluoromethylation of aryl halides. In some instances, co-catalysis with silver(I) has also been used to facilitate trifluoromethylation $\left(\right.$ Scheme $\left.76^{[353 \mathrm{~d}]}\right){ }^{[353 \mathrm{~d}, 361]}$

$$
\text { R= } \mathrm{NO}_{2}, \mathrm{Cl}, \mathrm{OPh}, \mathrm{NBn}_{2} \text {, etc. }
$$

Scheme 76. Cu and Ag co-catalyzed trifluoromethylation of aryl iodides with sodium trifluoroacetate

Trifluoromethylorganosilanes, reported first by Ruppert, ${ }^{[362]}$ are the most commonly used nucleophilic trifluoromethylation reagent in cross-coupling reactions and addition reactions. Trifluoromethylorganosilanes such as trimethylsilyltrifluoromethane $\left(\mathrm{TMSCF}_{3}\right)$ and triethylsilyltrifluoromethane $\left(\mathrm{TESCF}_{3}\right)$ can be desilylated with fluoride to give trifluoromethyl anion. The first report of copper-mediated trifluoromethylation of aryl iodides with $\mathrm{TMSCF}_{3} / \mathrm{KF}^{[363]}$ by Fuchikami laid the foundation for trifluoromethylation cross-coupling using trifluoromethyltrialkylsilanes. Aryl halides in conjunction with trifluoromethylorganosilanes can react with copper carbene complexes 4.1 and 4.2 described by Vicic (Scheme 77), ${ }^{[364]}$ copper/silver co-catalysts by Weng, Feng, and Huang, ${ }^{[361]}$ coppernitrogenous ligand catalysts by Amii, ${ }^{[365]}$ isolated stoichiometric copper-phenanthroline complexes by Hartwig (Scheme 78), ${ }^{[366]}$ and stoichiometric copper phosphine complexes by Grushin (Scheme 79) ${ }^{[367]}$ to afford trifluoromethylated arenes. The method by Hartwig and co-workers is the most functional group tolerant and allows for trifluoromethylation of electron-poor to electron-rich arenes and even ortho,ortho-disubstituted arenes while the catalytic system by Amii utilizes only $10 \mathrm{~mol} \%$ copper catalyst loading to afford trifluoromethylated arenes in $44-99 \%$ yield.

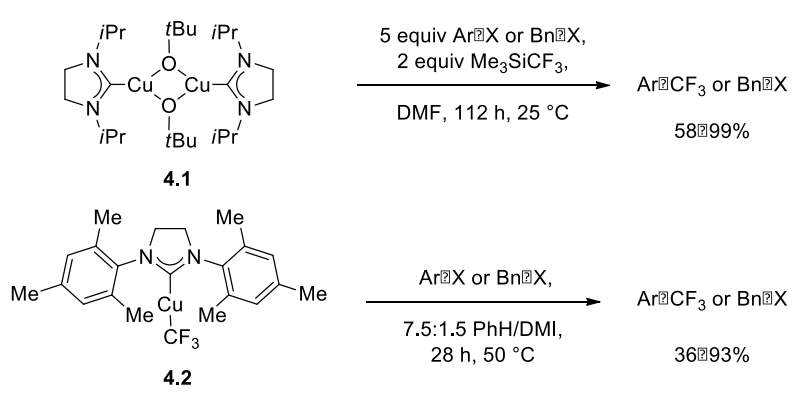

Scheme 77. Cu-mediatated trifluoromethylation of organic halides with $\mathrm{TMSCF}_{3}$

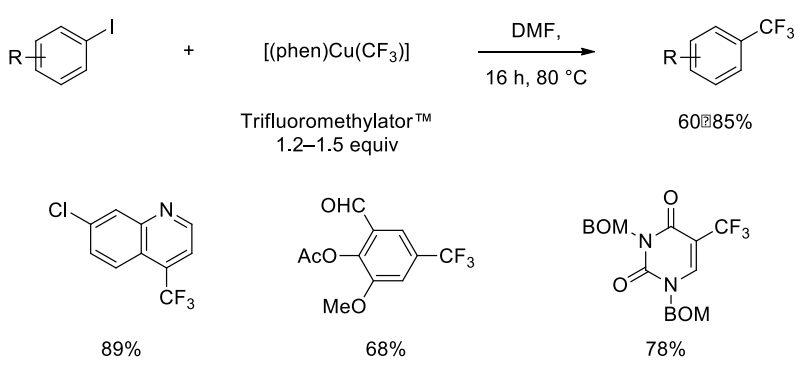

Scheme 78. Trifluoromethylation of aryl halides with Trifluoromethylator ${ }^{\mathrm{TM}}$

$$
\begin{aligned}
& 1.1 \text { equiv } \\
& \mathrm{R}=\mathrm{NO}_{2}, \mathrm{Me}, \mathrm{OMe}_{\mathrm{Cl}} \mathrm{CF}_{3} \text {, etc. }
\end{aligned}
$$

Scheme 79. Trifluoromethylation of aryl halides with Cu-phosphine trifluoromethyl complex

Copper-catalyzed trifluoromethylation of aryl iodides with trifluoromethylborate salts (Scheme 80$)^{[368]}$ and with $\alpha$ trifluoromethylmorpholinotrimethylsilylether (fluoral hemiaminal) (Scheme 81$)^{[369]}$ have been established in efforts to replace the use of volatile and moisture-sensitive trifluoromethyltrialkylsilanes. Trifluoromethylborate salts are crystalline, air-stable, storage-stable reagents that can act as a donor of trifluoromethyl anion in the presence of copper salts. ${ }^{[368]}$ Similarly, fluoral hemiaminal has been shown to be a stable and inexpensive source of trifluoromethyl anion; however, two equivalents of the reagent relative to the substrate are required. ${ }^{[370]}$ This necessity is postulated to arise from dimerization of the reagent to a silicate species that then acts as the reactive trifluoromethyl anion donor. ${ }^{[370 \mathrm{~b}]}$

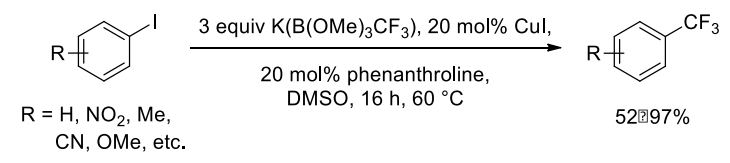

Scheme 80. Cu-catalyzed trifluoromethylation of aryl halides with potassium trifluoromethyltrimethoxyborate 


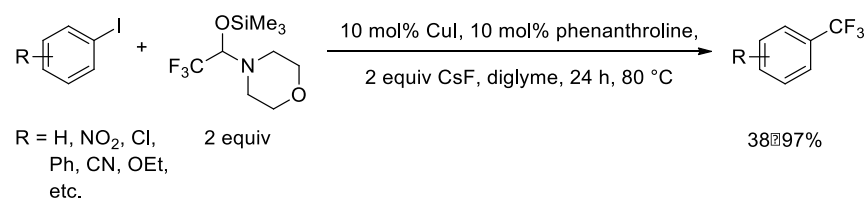

Scheme 81. Cu-catalyzed trifluoromethylation of aryl halides with fluoral hemiaminal

A Reformazky-elimination reaction sequence between methyl $\alpha$ trifluoromethyl- $\alpha, \alpha$-dichloroacetate with aldehydes mediated by zinc and copper(I) in acetic or trifluoroacetic anhydride afforded trifluoromethyl olefins (Scheme 82). ${ }^{[371]}$ Additionally, alkenyl bromides treated with nucleophilic trifluoromethyl copper complexes generated from fluorosulfonyldifluoroacetate (Scheme 83), ${ }^{[372]}$ mercury bis(trifluoromethyl), ${ }^{[373]}$ and $\mathrm{TMSCF}_{3}{ }^{[374]}$ can afford the desired trifluoromethyl alkenes.

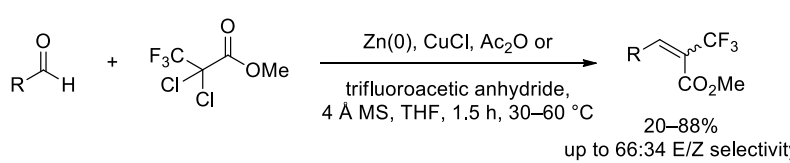

Scheme 82. Cu-mediated Reformazky-elimination reaction sequence to afford trifluoromethylated alkenes

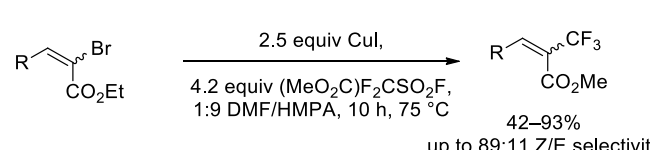

Scheme 83. Cu-mediated trifluoromethylation of alkenyl bromides

The strong $\mathrm{Pd}-\mathrm{CF}_{3}$ bond in trifluoromethyl palladium complexes renders reductive elimination of trifluoromethylarenes difficult compared to reductive elimination from aryl methyl palladium complexes, which readily undergo reductive elimination to afford toluene. ${ }^{[375]}$ The viability of reductive elimination from $\mathrm{Pd}(\mathrm{II})$ aryl complexes to form aryl- $\mathrm{CF}_{3}$ bonds was established by Grushin with chelating ligand XantPhos at $80{ }^{\circ} \mathrm{C}$ (Scheme 84, complex 4.3). ${ }^{[376]}$ Palladium-catalyzed cross-coupling of aryl chlorides with $\mathrm{TESCF}_{3}$ was achieved by Buchwald and co-workers using BrettPhos, a bulky monodentate phosphine, which allows reductive elimination to occur from the T-shaped trifluoromethyl aryl palladium phosphine complex (Scheme 85 ). ${ }^{[377]}$ While protic functional groups are not tolerated, possibly due protonation of trifluoromethyl anion to generate fluoroform, electron-rich and poor aryl chlorides as well as heteroaryl chlorides can be trifluoromethylated.

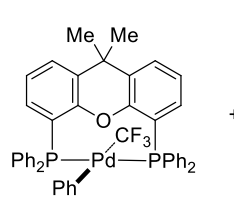

4.3

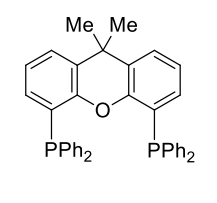

Xantphos $\left[(\text { Xantphos })_{2} \mathrm{Pd}\right]$

$\stackrel{\mathrm{PhH}, 3 \mathrm{~h}, 80^{\circ} \mathrm{C}}{\longrightarrow}$

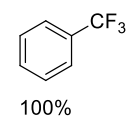

Scheme 84. Reductive elimination of trifluoromethyl arenes from Pd(II) complex

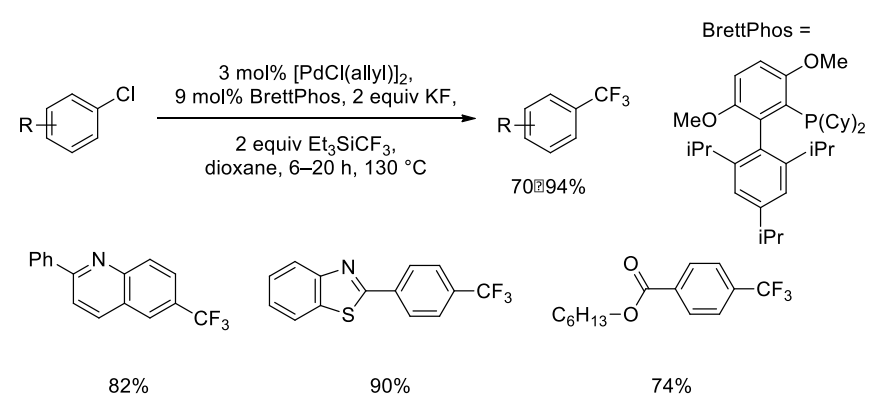

Scheme 85. Pd-catalyzed cross-coupling of aryl halides with $\mathrm{TMSCF}_{3}$

Cross-coupling with $\mathrm{TMSCF}_{3}$ and alkenyl sulfonates has been developed with palladium catalysts to afford trisubstituted trifluoromethyl alkenes (Scheme 86). ${ }^{[378]}$ An unconventional approach to installing trifluoromethylalkenyl groups on arenes uses palladium-catalyzed cross-coupling and $\beta$-hydride elimination to selectively afford disubstituted $E$-trifluoromethyl alkenes (Scheme 87). ${ }^{[379]}$ Electrophilic trifluoromethylation of alkynes with Togni reagent II mediated by copper iodide can afford trisubstituted trifluoromethyl alkenyl carbonates. ${ }^{[380]}$ For the synthesis of $\beta$ trifluoromethyl styrenes, aryl iodides underwent cross-coupling with E-trimethylsilyl-3,3,3-trifluoropropene with retention of doublebond geometry. ${ }^{[381]}$

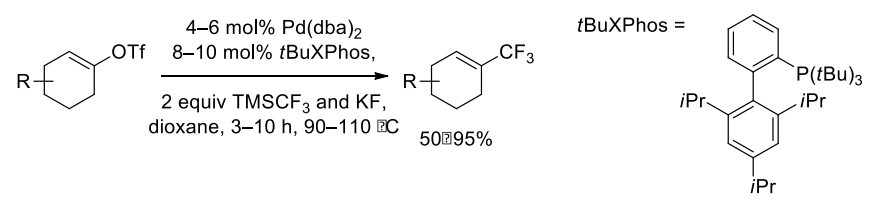

Scheme 86. Pd-catalyzed trifluoromethylation of alkenyl triflates

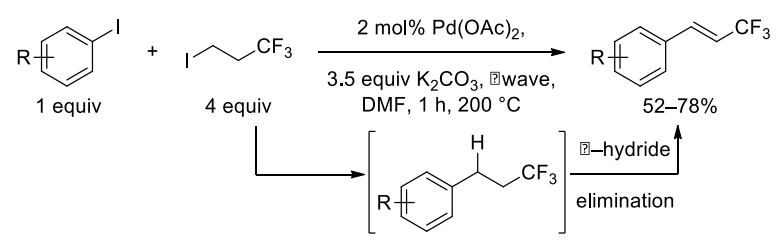

Scheme 87. Pd-catalyzed cross-coupling of aryl iodides and 1,1,1trifluoro-3-iodopropane with sequential $\beta$-hydride elimination

\subsubsection{Nucleophilic methods for the synthesis of trifluoromethylated $s p^{3}$ carbon centers}

Trifluoromethylating reagents of mecury, ${ }^{[382]}$ zinc, ${ }^{[226 a, 226 c, 383]}$ and cadmium, ${ }^{[26 a, 384]}$ were explored but difficulties and inefficient processes for reagent generation have made their use impractical. Currently, trifluoromethylorganosilanes are the most commonly used nucleophilic trifluoromethyl reagents in the laboratory setting.

Trifluoromethyltrimethylsilane $\left(\mathrm{TMSCF}_{3}\right)$ after desilylation with tetrabutylammonium fluoride (TBAF) or tris(dimethylamino)sulfonium difluorotrimethylsilicate (TASF) can react with aldehydes, ${ }^{[385]}$ esters, ${ }^{[386]} \mathrm{N}$-(tertbutylsulfinyl)imines, ${ }^{[387]}$ amino $N$-(tertbutylsulfinyl)imines (Scheme 88$),{ }^{[388]} N$-tosyl aldimines,${ }^{[389]}$ diketo compounds, ${ }^{[390]} \alpha, \beta$-unsaturated carbonyls, ${ }^{[391]}$ imines/iminiums, ${ }^{[392]}$ and isoxazoles.(Scheme 89$)^{[393]}$ The fluoride 
source is integral to constructive trifluoromethylation reactivity especially for enolizable substrates. Basic metal fluorides can $\alpha$ deprotonation enolizable substrates so tetraalkylammonium fluorides have been used because they exhibit the right amount of nucleophilicity and basicity. Other nucleophilic trifluoromethylation methods of carbonyls and carbonyl derivatives have been accomplished with Lewis acids and bases ${ }^{[258 e, 394]}$ as well as tri-tertbutylphosphine as the desilylating reagent. ${ }^{[395]}$

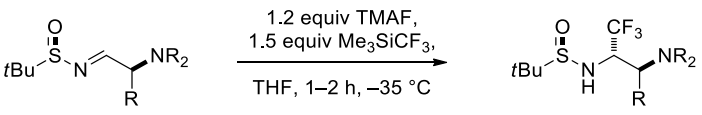

$$
\begin{aligned}
& \begin{array}{ll}
\mathrm{R}=\text { alkyl, } \mathrm{Bn} & 60-86 \% \\
& 80-99 \% \mathrm{~d}
\end{array}
\end{aligned}
$$

Scheme 88. Diastereoselective addition of $\mathrm{TMSCF}_{3}$ to $\mathrm{N}$ (tertbutylsulfinyl)imines

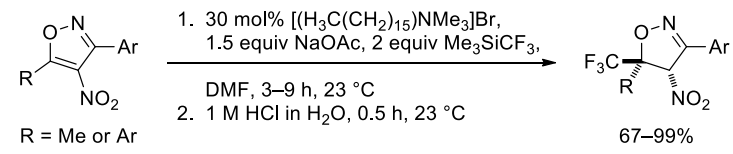

Scheme 89. Diasteroselective addition of $\mathrm{TMSCF}_{3}$ to isoxazoles

Preliminary efforts toward enantioselective trifluomethyl group addition reactions involved chiral amine auxiliaries such as $\mathbf{4 . 4}$ (Scheme 90$)^{[396]}$ or asymmetric induction chiral sulfinyl directing groups. ${ }^{[397]}$ The enantioselective addition of trifluoromethyl anion to carbonyl electrophiles has been challenging to accomplish because tight ion pairing between the chiral catalyst and the substrate has been hard to achieve. The use of chiral ammonium cinchona alkaloid-based catalysts such as $\mathbf{4 . 5}$ has enabled the asymmetric trifluoromethylation of aromatic aldehydes and ketones, ${ }^{[398]}$ cyclic electron-neutral aromatic ketones (Scheme 91), ${ }^{[399]}$ and alkynyl ketones. ${ }^{[400]}$ In place of the hydroscopic chiral ammonium fluoride salts, chiral ammonium bromides with exogenous potassium fluoride can be employed $;{ }^{[401]}$ however, because potassium fluoride is sparingly soluble in polar aprotic solvents, phenoxide was investigated as the desilylating agent for $\mathrm{TMSCF}_{3}{ }^{[402]}$ Beyond carbonyl substrates, activated azomethine imines have similarly been reported as viable substrates for enantioselective trifluoromethylation with a chiral ammonium bromide catalyst $\mathbf{4 . 6}$ (Scheme 92). ${ }^{[403]}$

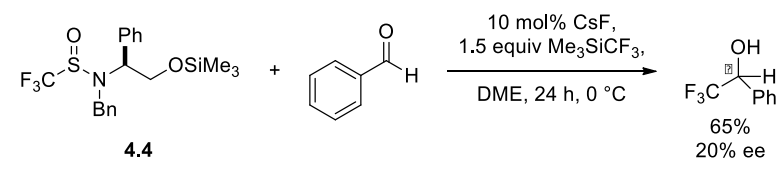

Scheme 90. Synthesis of enantioenriched trifluoromethylated alcohols with chiral amine auxiliary

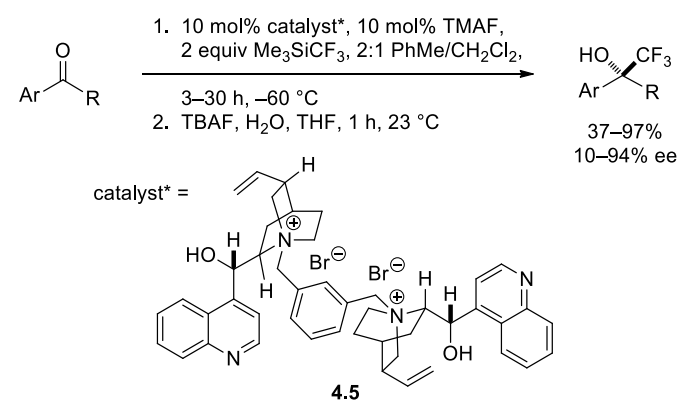

Scheme 91. Enantioselective addition of $\mathrm{TMSCF}_{3}$ to aryl ketones using PTC

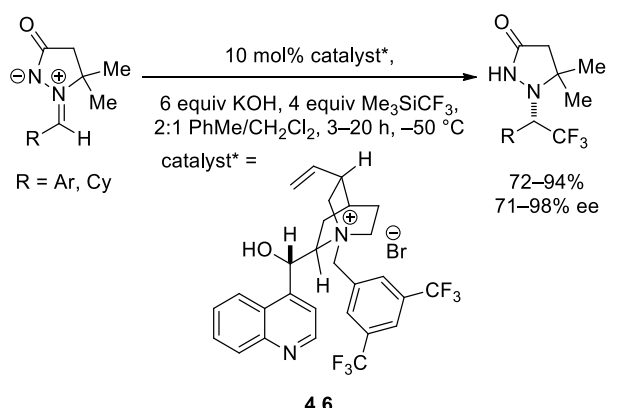

Scheme 92. Enantioselective addition of $\mathrm{TMSCF}_{3}$ to azomethine imines using PTC

Other nucleophilic trifluoromethylating reagents such as $\alpha$ trifluoromethylmorpholinotrimethylsilylether, ${ }^{[370 b]} \quad \alpha$ trifluoromethylpiperazinotrimethylsilylether, ${ }^{[370 \mathrm{a}, 404]}$ and potassium trifluoromethylborates ${ }^{[405]}$ have been utilized for trifluoromethylation reactions. Trifluoroiodomethane reduced with $\mathrm{TDAE}^{[406]}$ as well as copper in conjunction with methyl fluorosulfonyldifluoroacetate ${ }^{[407]}$ chlorodifluoroacetate,${ }^{[408]}$ methyl chlorodifluoroacetate ${ }^{[356,409]}$ bromotrifluoromethane, ${ }^{[410]}$ and sodium trifluoroacetate ${ }^{[411]}$ have been used to generate nucleophilic trifluoromethylating reagents. While many copper-based methods exist, further development with these copper reagents for substitution and addition reactions would need to be developed for practical and efficient reaction chemistry. More recently, $\left(\mathrm{Ph}_{3} \mathrm{P}\right)_{3} \mathrm{CuCF}_{3}$ complexes have been reacted with propargyl halides to afford halide displaced trifluoromethylation products ${ }^{[12]}$ and additionally copper salts in conjunction with TMSCF3 and CsF has been used for the trifluoromethylation of $\alpha$-diazo esters. ${ }^{[413]}$

Enantioenriched trifluoromethyl allyl compounds can be synthesized via enantioselective trifluoromethylation of BaylisHilman adducts of allylic acetates (Scheme 93) $)^{[414]}$ and carbonates. ${ }^{[415]}$ The substrate scope is limited to compounds containing an ester group beta to the allylic acetate or carbonate moiety. 


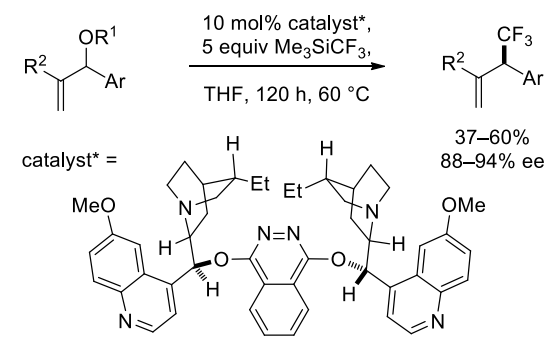

Scheme 93. Enantioselective synthesis of of allylic trifluoromethylated products via cinchona alkaloid catalysis

\section{Trifluoroethylation and perfluoroalkylation}

Direct introduction of trifluoroethyl groups for the synthesis of compounds, many of which find applications as pharmaceuticals. ${ }^{[416]}$ can be accomplished with trifluoromethylhalomethane using either nucleophilic (i.e. aryl iodide cross-coupling with trifluoromethyliodomethane) or electrophilic methods (i.e. arylboronic acids cross-coupling with trifluoromethyliodomethane). Different iodine-based electrophilic trifluoroethylating reagents have been developed for the trifluoroethylation of $\mathrm{N}-, \mathrm{O}-$, and $\mathrm{S}$ nucleophiles. Similarly, perfluoroalkylation reactions can occur with electrophilic perfluoroalkylating reagents such as perfluoroalkyliodonium salts or nucleophilic perfluoroalkylating reagents synthesized by reduction of perfluoroalkylhalides with copper, zinc, or organic reductants.

\subsection{Trifluoroethylation}

Trifluoroethylated arenes were first synthesized via nucleophilic substitution of the corresponding benzyl bromide with trifluoromethyl copper or bis(trifluoromethyl)mercury reagents (Scheme 94). ${ }^{[364,417]}$ A new operationally facile approach for the generation of trifluoromethyl copper complex has been reported by reduction of $S$-(trifluoromethyl)diphenylsulfonium triflate with copper metal at $60{ }^{\circ} \mathrm{C}$ to afford 36-83\% yield of trifluoroethylated arene products. $^{[418]}$

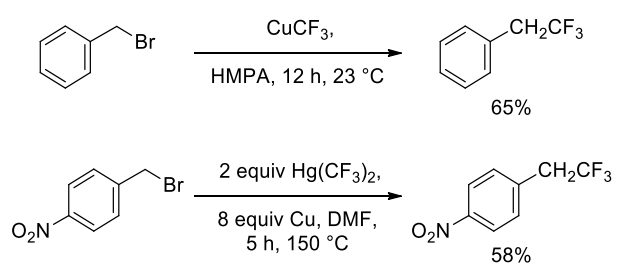

Scheme 94. Synthesis of trifluoroethylated arenes with nucleophilic trifluoromethylating reagents

Direct trifluoroethylation of iodobenzene was first reported by McLoughlin and Thrower through copper-mediated nucleophilic trifluoroethylation with 1-iodo-2,2,2-trifluoroethane (Scheme 95). ${ }^{[417 a]}$ Drawbacks include the need for excess aryl iodide and low yields of the trifluorethylated arenes. Subsequently, Hartwig reported the reductive elimination of para-trifluoroethyltoluene from an aryl trifluoroethyl $\mathrm{Pd}(\mathrm{II})$ complex, demonstrating the aryl $\mathrm{C}-\mathrm{CH}_{2} \mathrm{CF}_{3}$ bond could be formed by reductive elimination. ${ }^{[419]}$ Palladium-catalyzed cross-coupling of trifluoromethyliodomethane with aryl and alkenylboronic acids and esters (Scheme 96) ${ }^{[420]}$ or alkenyl stannanes ${ }^{[324]}$ affords trifluoroethylated arenes or alkenes. This cross-coupling method displays broad substrate scope and functional group tolerance, and allows for trifluoroethylation of $m e t a$-aminophenylboronic acid with no $N$-trifluoroethylated byproducts. Additionally, cross-coupling of boronic acids and esters with trifluoromethyliodomethane is complimentary to trifluoroethylations using aryl halides.

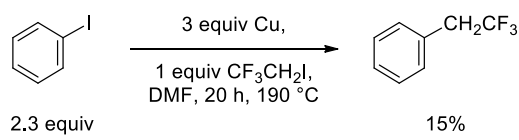

Scheme 95. Cu-mediated cross-coupling of aryl iodides with trifluoromethyliodomethane

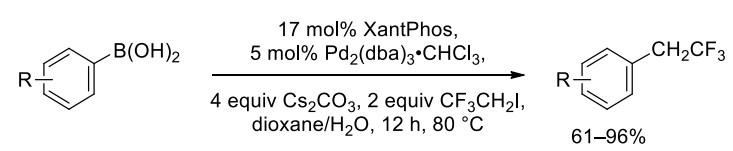

Scheme 96. Pd-catalyzed trifluoroethylation of arylboronic acids

Trifluorodihaloethylzinc nucleophiles generated from oneelectron reduction with zinc powder of 1,1,1-trihalo-2,2,2trifluoroethane reagents were used in aldehyde addition reactions. ${ }^{[421]}$ Electrophilic trifluoroethylation can occur via radical pathways with alkenes ${ }^{[422]}$ and alkynes ${ }^{[422 b]}$ using halogenated trifluoroethane reagents and radical initiators such as copper salts ${ }^{[422 \mathrm{a}]}$ or sodium dithionite (Scheme 97). ${ }^{[422 \mathrm{~b}]}$
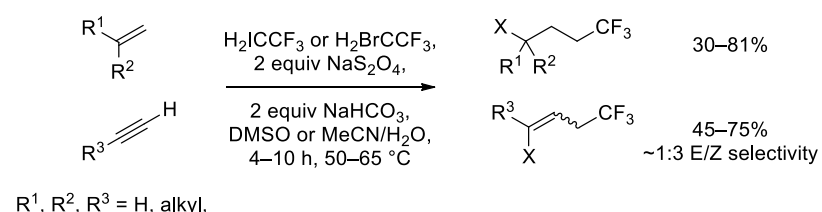

$\mathrm{CO}_{2} \mathrm{Me}$, etc

Scheme 97. Radical addition of trifluoromethylhalomethane across alkenes and alkynes

Nitrogenous nucleophiles such as aniline, amines (Scheme 98), imidazoles, and pyridines as well as phenols, alcohols, carboxylic acids, and thiols can be trifluoroethylated using trifluoroethyliodonium salts, ${ }^{[423]}$ which act as sources of electrophilic trifluoroethyl groups. ${ }^{[223 b,}$ 423c, 424] Carbon-based nucleophiles afford trifluoroethylated products in $25-36 \%$ yield, although $\alpha$-trifluoroethylation of silylenol ethers is possible in 49 $92 \%$ yield. $^{[425]}$ A broader range of non-aromatic $N$-, O-, and $S$ nucleophiles than reported in prior works can react with reagent $\mathbf{5 . 1}$ by trifluoroethylation of the heteroatom. ${ }^{[424 \mathrm{c}]}$

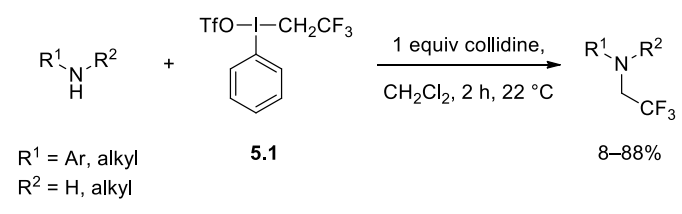

Scheme 98. Trifluoroethylation of amines with electrophilic trifluoroethylating hypervalent iodine reagents 


\subsection{Perfluoroalkylation}

Nucleophilic perfluoroalkyl copper reagents were first used to afford perfluoroalkyl arenes from aryl halides ${ }^{[417 a]}$ and perfluoroalkyl-substituted alkenes from alkenyl halides. ${ }^{[426]}$ When ionic liquids were used as solvent for perfluoroalkylation of aryl halides, reaction temperature could be lowered from $150{ }^{\circ} \mathrm{C}$ to $75{ }^{\circ} \mathrm{C} .{ }^{[427]}$ Perfluoroalkylation of alkenyl and aryl halides has been investigated with discrete phenanthroline-ligated perfluoroalkyl copper complexes by Hartwig (Scheme 99),${ }^{[366]}$ and with palladium catalysts and zinc metal under ultrasonic irradiation by Ishikawa. ${ }^{[428]}$ Chambers used sodium perfluoroalkylcarboxylates ${ }^{[429]}$ and Daugulis $1 \mathrm{H}$-perfluoroalkanes ${ }^{[343]}$ in lieu of perfluoroalkyl halides as sources of perfluoroalkyl groups to accomplish perfluoroalkylation. The method reported by Hartwig affords electron-rich and -poor perfluoroalkyl arenes and heteroarenes in $88-99 \%$ yield at $50{ }^{\circ} \mathrm{C}$ while the method developed by Daugulis can afford perfluoroalkylated products from 1H-perfluoroalkanes ranging from trifluoromethane to $1 \mathrm{H}$-perfluorodecane (Scheme 100). ${ }^{[343]}$ Arylboronic acids undergo electrophilic perfluoroalkylation with perfluoroalkyl halide and copper under ambient atmosphere. ${ }^{[242]}$ This room temperature perfluoroalkylation method by Shen and co-workers yields perfluoroalkylated arenes in $29-65 \%$ yield.

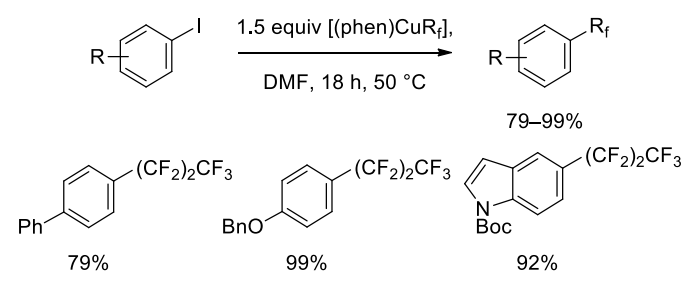

Scheme 99. Perfluoroalkylation of aryl iodides with $\mathrm{Cu}$ phenanthroline perfluoroalkyl complex

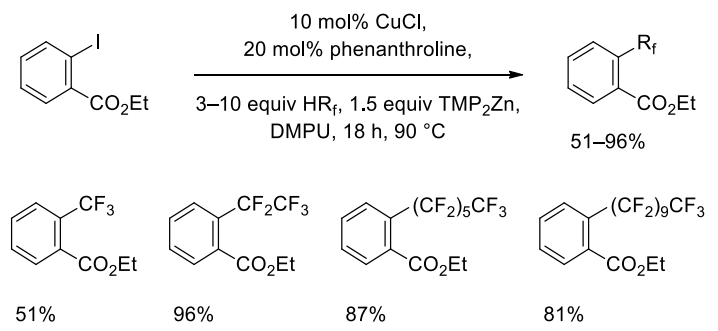

Scheme 100. Cu-catalyzed perfluoroalkylation of aryl iodides

A general strategy for the perfluoroalkylation of arenes has been reported by Hartwig and co-workers via a one-pot borylation/perfluoroalkylation sequence. ${ }^{[296 b]}$ Perfluoroalkylation of arenes can be accomplished with a palladium-BINAP catalyst and perfluoroalkyl halide as described by Sanford; solvent quantities of the arene are needed (Scheme 101). ${ }^{[330]}$

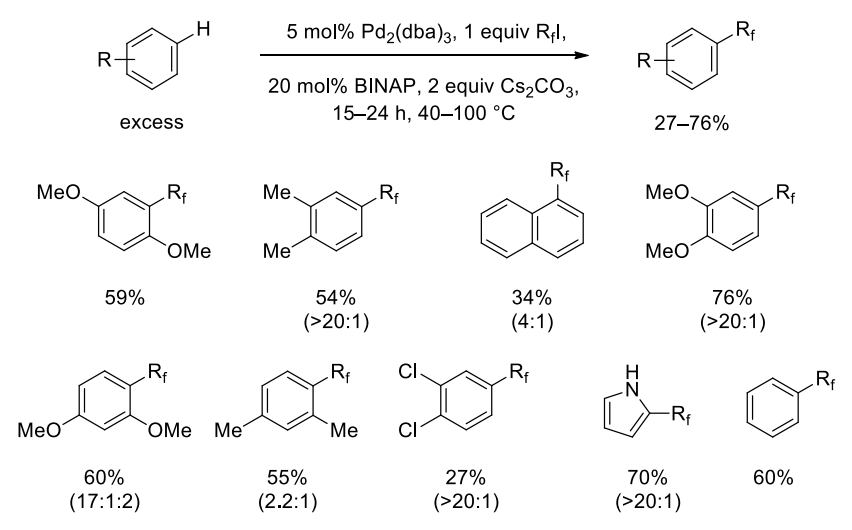

Scheme 101. Pd-catalyzed perfluoroalkylation of unactivated arenes with pefluoroalkyl iodide

Radical addition of haloperfluoroalkylsulfonyl halides across alkenes can be accomplished with benzoyl peroxide ${ }^{[431]}$ and ruthenium(II) catalysts. $^{[432]}$ Perfluoroalkyl transition metal complexes can react with alkynes, ${ }^{[433]}$ dienes, and allylic halides, ${ }^{[434]}$ as well as propargyl halides ${ }^{[435]}$ to afford the corresponding perfluoroalkylated alkenes and allenes. Nucleophilic reagents, such as perfluoroalkyl lithium ${ }^{[436]}$-magnesium bromide,${ }^{[437]}$-calcium, ${ }^{[438]}$ tin, ${ }^{[439]}$ trimethylsilane ${ }^{[440]}$ and -zinc (with a palladium or nickel catalyst), ${ }^{[434,441]}$ were used prior to the development of the TDAE/perfluoroalkyl halide combination by Dolbier, which is a straightforward and practical method for the perfluoroalkylation of aldehydes, ketones, imines, disulfides, and diselenides (Scheme 102) ${ }^{[422]}$ affording nonafluorobutylation products in $20-98 \%$ yield. Similarly, Prakash reported the alkoxide-induced release of perfluoroalkyl anion from pentafluoroethylphenylsulfone reagents which reacts with carbonyls and imines in $50-99 \%$ yield (Scheme 102). ${ }^{[443]}$

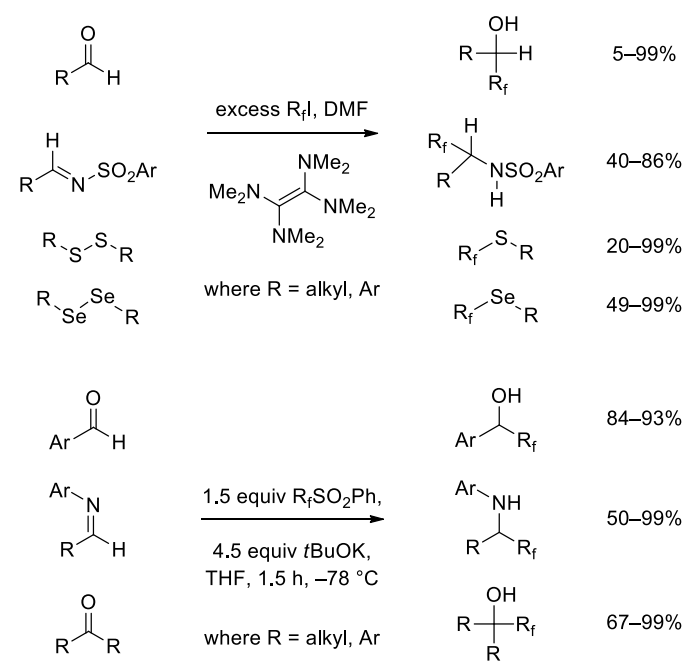

Scheme 102. Two methods for the addition of perfluoroalkyl groups to carbonyl and carbonyl derivatives

Aryl perfluoroalkyliodonium chloride reagents were developed for perfluoroalkylation of thio- and seleno-nucleophiles by Yagupolskii. ${ }^{[44]}$ The chloride was subsequently exchanged for the less coordinating triflate counterion resulting in the more electrophilic FITS (perfluoroalkylphenyliodonium trifluoromethanesulfonate) reagent for perfluoroalkylation (Scheme 
103) of carbanion nucleophiles, ${ }^{[45]}$ arenes, ${ }^{[446]}$ thiophenols and thiols at sulfur, ${ }^{[335]}$ substituted phenols at oxygen, ${ }^{[47]}$ alkynes, ${ }^{[48]}$ and alkylenol ethers at carbon ${ }^{[449]}$ as described by Umemoto. Additionally, alkenes can be perfluoroalkylated with the FITS reagent to afford perfluoroalkyl olefins. ${ }^{[450]} \alpha$-Perfluoroalkylation of silylenol ethers with perfluoroalkyl halides can be initiated with radical promoter triethylborane. ${ }^{[451]}$ Another approach for the $O$ - and $S$-perfluoroalkylation of phenolates and thiophenolates involves the use of tetrafluorodihaloethane and perfluorodihaloalkanes. ${ }^{[452]}$ This process is proposed to occur via in situ conversion of the perfluoroalkylating reagent to a perfluorinated alkene intermediate which is supported by separate reports of $O$-perfluoroalkylation at phenol with perfluoropropylene ${ }^{[453]}$ and $N$-perfluoroalkylation with tetrafluoroethylene. $^{[454]}$

In addition to perfluoroalkyl substituents, the pentaflurosulfanyl substituent has received increased attention over the recent past Pentafluorosulfanyl substituents are chemically inert, increase lipophilicity, and could develop as useful groups for pharmaceuticals and agrochemicals. ${ }^{[455]}$ Simple pentafluorosulfanyl-substituted compounds are commercially available, but the difficulty of synthesis and requirement for toxic reagents is currently a drawback for the development of new methods for incorporation of the $\mathrm{SF}_{5}$ group into more complex substrates.

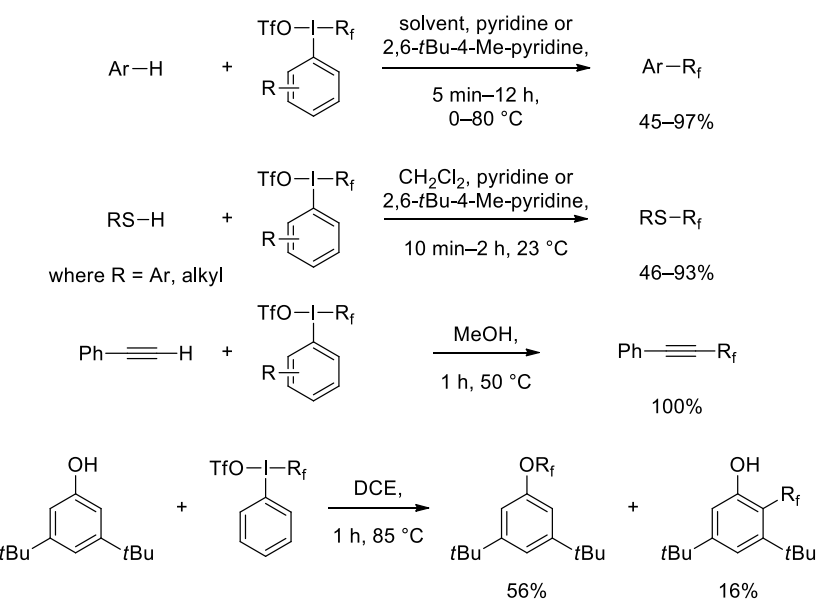

Scheme 103. Perfluoroalkylation of arenes, sulfides, terminal alkynes, and 3,5-ditert-butylphenol with electrophilic perfluoroalkyl hypervalent iodine reagents

\section{Trifluoromethyl ethers, sulfides, and amines}

Trifluoromethoxylated and trifluoromethylthiolated molecules have been used as agrochemicals, pharmaceuticals, and electrooptical materials. ${ }^{[456]}$ The increase in lipophilicity ${ }^{[457]}$ resulting from trifluoromethoxy and trifluoromethylthiol group incorporation has made both functional groups attractive substituents for use in medicinal chemistry. Additionally, the ability to induce conformational changes through the trifluoromethoxy substituent, which adopts an orthogonal orientation with respect to the arene plane, ${ }^{[2]}$, in contrast to a methoxy group, which normally rests in plane of the arene, can be beneficial to obtain additional binding affinity. Trifluoromethylamines have applications as fluorinating reagents ${ }^{[458]}$ these compounds are prone to fluoride ionization followed by hydrolysis of the resulting iminium or imine, which renders many trifluoromethylamines difficult to isolate and store.

The difficulty of trifluoromethylation at heteroatoms is dependent on the nucleophilicity of the heteroatom; trifluoromethylation at nitrogen and oxygen is more difficult to accomplish than at sulfur. ${ }^{[459]}$ Consequently, the synthesis of trifluoromethyl ethers and amines has classically involved prefunctionalization at oxygen or nitrogen with carbonyl or thiocarbonyl functional groups followed by fluorine introduction. Many approaches for the synthesis of trifluoromethyl ethers and amines have low functional group tolerance due to the use of hydrogen fluoride, Lewis acids, or thermally unstable, reactive trifluoromethylating reagents. Trifluoromethylsulfides can be more easily synthesized using a variety of electrophilic trifluoromethylating reagents. Additionally, the enhanced stability and nucleophilicity of free trifluoromethylthiolate as compared to trifluoromethoxide, which eliminated fluoride at elevated temperature, has facilitated the development cross-coupling methods for the synthesis of trifluoromethylsulfides.

\subsection{Preparation of trifluoromethyl ethers}

The formation of the oxygen- $\mathrm{CF}_{3}$ bond as well as the carbon$\mathrm{OCF}_{3}$ bond has been difficult to accomplish for two main reasons: reactivity of the trifluoromethylating reagents with carbon sites of the oxygenated nucleophile leading to formation of byproducts and the thermal instability of many trifluoromethoxide salts. Many nucleophilic fluorodesulfurization and fluorodeoxygenation of functionalized alcohols and phenols have been established for the synthesis of trifluoromethyl ethers whereas fewer methods for the formation of oxygen- $\mathrm{CF}_{3}$ and carbon- $\mathrm{OCF}_{3}$ bonds have been successfully accomplished. Synthesis of aryl trifluoromethyl ethers via O-trifluoromethylation of phenols is currently of limited synthetic use due to carbon trifluoromethylation or the need for impractical reaction conditions with thermally unstable $O$ (trifluoromethyl)dibenzofuranium reagents. In contrast, $O$ trifluoromethylation of primary and secondary alcohols with thermally stable Togni reagent affords the desired trifluoromethyl ethers. $^{[460]}$

\subsubsection{Preparation of aryl trifluoromethyl ethers}

Nucleophilic fluorination of aryl trichloromethyl ethers, ${ }^{[461]}$ aryl chlorothionoformates, ${ }^{[461 \mathrm{~b}, 462]}$ phenyl fluoroformates, ${ }^{[463]}$ and aryl xanthates $^{[464]}$ constitute most of the methods developed for the synthesis of trifluoromethyoxyarenes. A general, practical, functional-group-tolerant trifluoromethylation of phenols is not yet available. Aryl trichloromethyl ethers ${ }^{[461 b, 461 d]}$ synthesized in situ $^{[461 \mathrm{e}]}$ or prior to the reaction by chlorination of the aryl methyl ether $^{[461 \mathrm{a}, 461 \mathrm{c}, 461 \mathrm{f}]}$ or aryl chlorothionoformates ${ }^{[461 \mathrm{~b}, 462]}$ can be nucleophilically fluorinated with hydrogen fluoride, ${ }^{[461 e}$, 465$]$ $\mathrm{SbF}_{5},{ }^{[461 \mathrm{~g}]} \mathrm{SbF}_{3}$ in the presence of $\mathrm{SbF}_{5},{ }^{[461 \mathrm{a}, 461 \mathrm{~b}, 466]}$ or molybdenum hexafluoride. ${ }^{[462,467]}$ Likewise, phenol fluoroformates can be treated with $\mathrm{SbF}_{3}$ or $\mathrm{SF}_{4} / \mathrm{HF}$ to afford trifluoromethoxyarenes (Scheme 104) ${ }^{[463]}$ Aryl xanthates were used as substrates for fluorodesulfurization with $\mathrm{HF} /$ pyridine and dibrominated hydantoin (Scheme 105). ${ }^{[464 a]}$ 


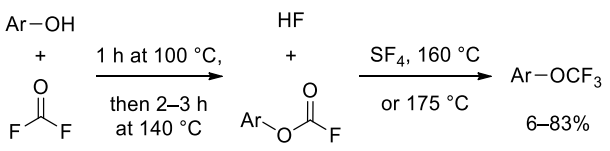

$$
\begin{aligned}
& \text { where } \mathrm{Ar}=\mathrm{Ph}, \mathrm{o}-\mathrm{NO}_{2}-\mathrm{Ph}, 2 \text {,4-diBr-Ph, } m-\mathrm{OH}-\mathrm{Ph}, p-\mathrm{Cl}-\mathrm{Ph} \text {, etc. }
\end{aligned}
$$

Scheme 104. Synthesis of aryl trifluoromethyl ethers via fluoroformate intermediates

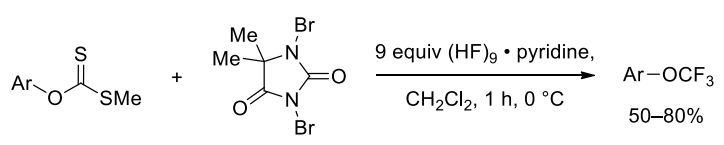

Scheme 105. Synthesis of aryl trifluoromethyl ethers by fluorodesulfurization of aryl xanthates

Umemoto reported that phenol derivatives can undergo trifluoromethylation with $O$-(trifluoromethyl)dibenzofuranium reagents in the presence of alkyl amine bases to afford trifluoromethoxyarenes (Scheme 106). ${ }^{[459]}$ The reagent needs to be generated prior to use by photochemical decomposition of the trifluoromethyoxybiaryl diazonium salt at -100 to $-90{ }^{\circ} \mathrm{C}$ to yield the active $O$-(trifluoromethyl)dibenzofuranium reagent $\mathbf{6 . 1}$. Trifluoromethylation of phenols with Togni reagent II was investigated by Togni and co-workers, and $O$-trifluoromethylation occurred with 2,4,6-trimethylphenol in $15 \%$ yield along with carbon trifluoromethylation products (Scheme 107). ${ }^{[273 b]}$

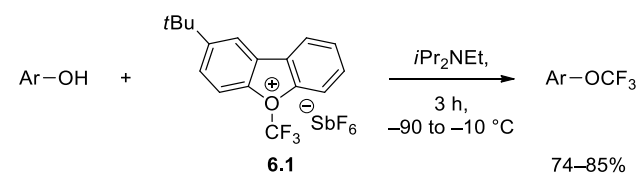

Scheme 106. O-Trifluoromethylation of phenols to afford aryl trifluoromethyl ethers

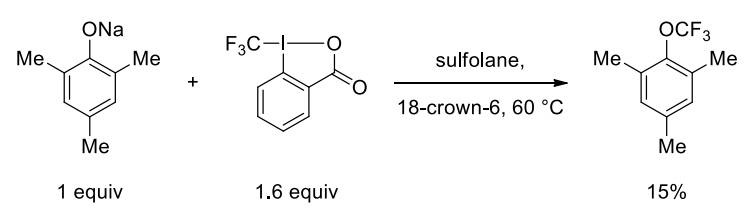

Scheme 107. O-Trifluoromethylation of phenols with Togni reagent II

The use of trifluoromethoxide anion to form the aryl carbonoxygen bond has not been as widely explored as other approaches. $\mathrm{S}_{\mathrm{N}} \mathrm{Ar}$ reaction with trifluoromethoxide was attempted, but products from $S_{N} A r$ with fluoride are observed instead, likely due to trifluoromethoxide degradation into volatile carbonyl difluoride and fluoride ${ }^{[468]}$ Kolomeitsev reported that phenyl and naphthyl carbon$\mathrm{OCF}_{3}$ formation occurred when trifluoromethoxide anion was added to benzynes to afford aryl trifluoromethyl ethers (Scheme 108). ${ }^{[468]}$ Aryl stannanes and aryl boronic acids can undergo silver-mediated cross-coupling with tris(dimethylamino)sulfonium trifluoromethoxide to afford functionalized aryl trifluoromethyl ethers (Scheme 109). ${ }^{[469]}$ This method affords the cross-coupling products of functionalized aryl stannanes and boronic esters with trifluoromethoxide; heteroaryl nucleophiles and nucleophilic amines cannot currently be tolerated. More recently, copper and gold oxygen-bound trifluoromethoxide complexes have been prepared and characterized in efforts to provide better understanding of transition metal interactions with trifluoromethoxide anion. ${ }^{[470]}$ Preliminary efforts toward the direct $\mathrm{C}-\mathrm{H}$ functionalization of arenes with trifluoromethylhypofluorite has been reported but mixtures consisting mainly of fluorinated arenes in addition to trifluoromethoxylated arenes. ${ }^{[471]}$

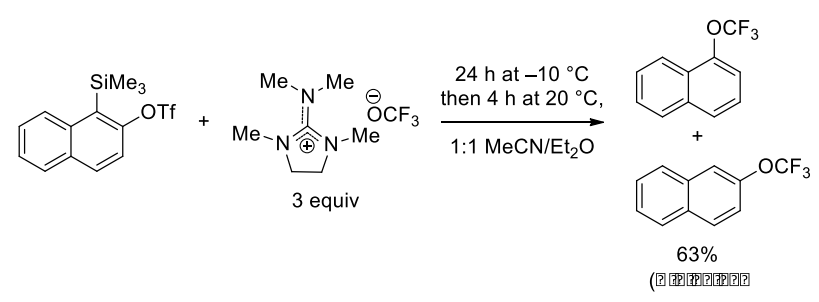

Scheme 108. Trifluoromethoxide anion addition to orthotrimethylsilylnaphthyl triflate via aryne intermediate
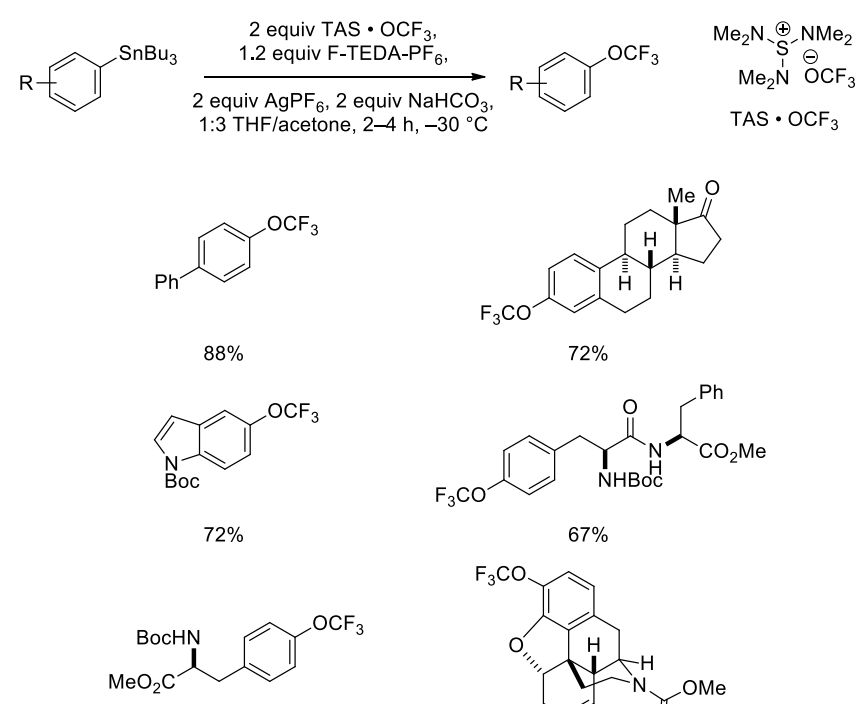

$75 \%$

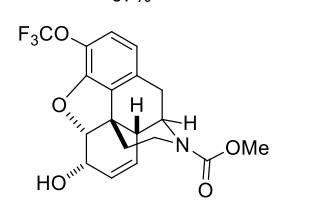

$59 \%$

Scheme 109. Ag-mediated cross-coupling of aryl stannanes with trifluoromethoxide salt

\subsubsection{Preparation of alkyl trifluoromethyl ethers}

Alkyl trifluoromethyl ethers can be synthesized via the trifluoromethylation of alcohols or displacement of a leaving group on alkyl electrophiles by trifluoromethoxide anion. Primary and secondary alcohols can be trifluoromethylated with Togni reagent in the presence of $\mathrm{Zn}\left(\mathrm{NTf}_{2}\right)_{2}$ (Scheme 110). ${ }^{[460]}$ The soluble zinc catalyst with non-nucleophilic triflimide anions is proposed by Togni and co-workers to activate the reagent for attack through coordination to the carboxy group of the reagent. Togni reagent II reacts with tetrahydrofuran in the presence of Lewis or Brønsted acids to afford polymeric ring-opened trifluoromethyl ethers ${ }^{[460 \mathrm{~b}]}$ and reacts with sulfonic acids to afford $O$-trifluoromethylated sulfonic acids. ${ }^{[340]}$ 2-Phenylethanol, $n$-decanol, and (2naphthyl)methanol were reported by Umemoto to react with $O$ (trifluoromethyl)dibenzofuranium reagents (shown in Scheme 106 with phenols) to afford the corresponding alkyl trifluoromethyl ethers. ${ }^{[459]}$ Analogous to the fluorination of aryl xanthates, ${ }^{[464 a]}$ alkyl 
xanthates were treated with $\mathrm{BrF}_{3}$ to afford alkyl trifluoromethyl ethers $^{[464 b]}$ while alkyl triflates were substituted with trifluoromethoxide anion (Scheme 111). ${ }^{[468]}$

$$
\begin{aligned}
& \text { R-OH } \\
& \begin{array}{ll}
5 \text { equiv } & 1 \text { equiv } \quad 12-99 \%
\end{array}
\end{aligned}
$$

Scheme 110. O-Trifluoromethylation of alcohols with Togni reagent II

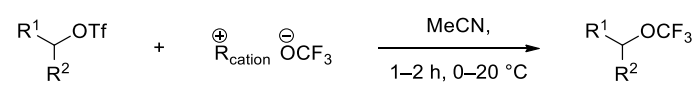$$
57-91 \%
$$

$\underbrace{\mathrm{O}}_{\mathrm{OCF}_{3}}$<smiles>CCOC(=O)C(OC(F)(F)F)c1ccccc1</smiles><smiles>O=C1c2ccccc2C(=O)N1CCOC(F)(F)F</smiles>

$87 \%$

$57 \%$<smiles>CC(=O)OCc1ccc(C(F)(F)F)cc1</smiles>

$87 \%$
Scheme 111. Synthesis of trifluoromethyl ethers via displacement of alkyl triflates with trifluoromethoxide salts

\subsection{Preparation of trifluoromethyl sulfides}

Alkylation of thiols with various electrophilic trifluoromethylating reagents has been a fruitful approach toward the synthesis of trifluoromethylsulfides. The S-trifluoromethylation of a broad range of aliphatic thiols and thiophenols (Scheme $112)^{[273 a]}$ and $S$-hydrogen phosphorothiolates ${ }^{[472]}$ was reported by Togni and co-workers using Togni reagent II and later applied to the trifluoromethylation of cysteine residues in peptide substrates. ${ }^{[473]}$ $\mathrm{Se}$-(trifluoromethyl)dibenzoselenophenium reagent $\mathbf{6 . 2}$ can likewise afford $S$-trifluoromethylated thiols as demonstrated by Umemoto (Scheme 113). ${ }^{[271 b]}$

$$
\text { R=Ar, alkyl }
$$

Scheme 112. S-Trifluoromethylation of thiols with Togni reagent II

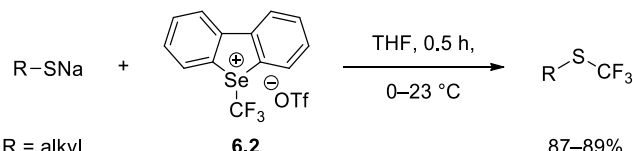

Scheme 113. S-Trifluoromethylation of sodium thiolates with S(trifluoromethyl)dibenzoselenophenium triflate

Trifluoromethylsulfides can be synthesized by photolysis of aryl and alkyl trifluoromethylthiosulfonates ${ }^{[474]}$ to promote desulfonylation and radical recombination or trifluoromethylation of disulfides ${ }^{[475]}$ with $\mathrm{TMSCF}_{3}$ as reported by Langlois. Similarly, disulfides can be trifluoromethylated with a mixture of TDAE and iodotrifluoromethane (Scheme 114). ${ }^{[476]}$ The $\mathrm{CF}_{3} \mathrm{I} / \mathrm{TDAE}$ method developed by Dolbier can trifluoromethylate both sulfur atoms of simple disulfides in 65-99\% yield. Cysteine derivatives have been trifluoromethylated with sodium trifluoromethylsulfinate and tertbutyl hydrogen peroxide (Scheme 115). ${ }^{[477]}$ Trifluoromethanesulfanamide reagents have also been used as electrophilic source of " $\mathrm{SCF}_{3}$ " groups with aryl and alkyl Grignard nucleophiles for the synthesis of trifluoromethyl sulfides (Scheme 116). ${ }^{[478]}$

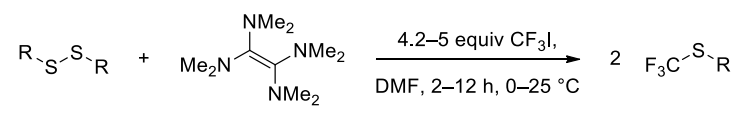

$$
\begin{aligned}
& \mathrm{R}=\mathrm{Ar} \text {, alkyl } 2.2 \text { equiv } \quad 65-99 \%
\end{aligned}
$$

Scheme 114. S-Trifluoromethylation of disulfides

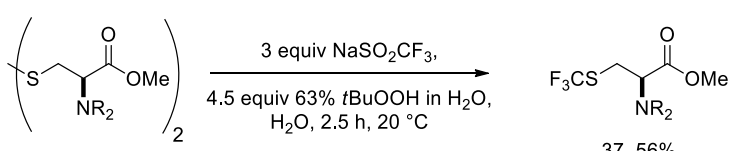

$$
\begin{aligned}
& \text { (only one sulfur equiv) }
\end{aligned}
$$

Scheme 115. S-Trifluoromethylation of cysteine derivatives

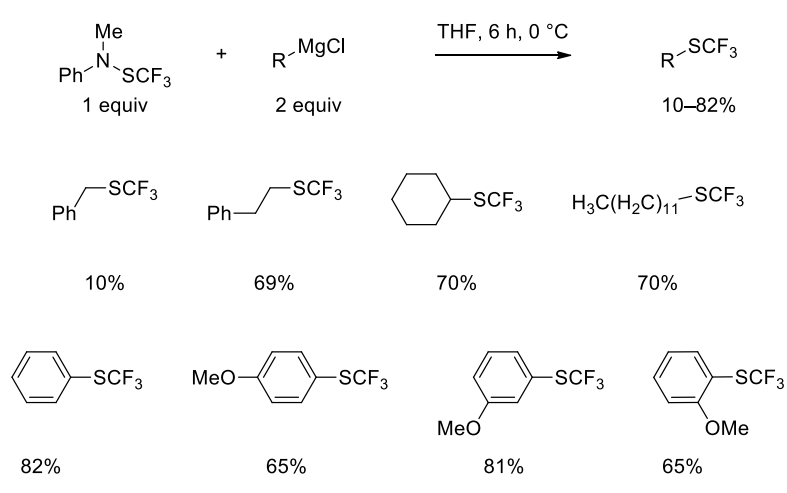

Scheme 116. Grignard reaction with trifluoromethanesulfanamide reagent to afford trifluoromethylsulfides

Displacement of chloride in $\mathrm{S}_{\mathrm{N}} \mathrm{Ar}$ reactions of electron-poor chloroarenes with silver trifluoromethylthiolate is limited in substrate scope but affords the desired aryl trifluoromethylsulfide (Scheme 117). ${ }^{[479]}$ Analogous to the synthesis of aryl trifluoromethyl ethers, ortho-trimethylsilylphenyl trifluoromethanesulfonate can be used to generate a benzyne intermediate that reacts with TDAEbis(trifluoromethylthiolate) to give phenyl trifluoromethylsulfide (Scheme 118). ${ }^{[468]}$ Although both approaches give rise to trifluoromethylsulfides in high yield, the reported methods are biased toward electron-poor arenes or unfunctionalized orthotrimethylsilylaryl triflates.

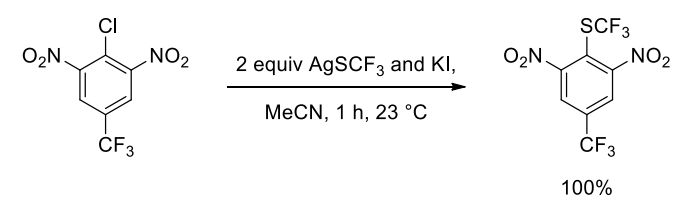

Scheme 117. Nucleophilic aromatic substitution with silver trifluoromethylthiolate 


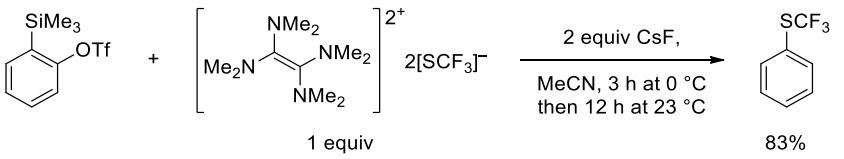

Scheme 118. Trifluoromethylthiolate anion addition to orthotrimethylsilylphenyl triflate via aryne intermediate

Aryl trifluoromethyl sulfides were synthesized from aryl diazonium salts (Scheme 119) ${ }^{[480]}$ and electron-poor aryl iodides (Scheme 120$)^{[481]}$ by Clark via reaction with pre-generated copper trifluoromethylthiolate. Chen has reported the in situ generation of copper trifluoromethylthiolate at $100{ }^{\circ} \mathrm{C}$ from copper, octathiocane (sulphur, $\mathrm{S}_{8}$ ), and fluorosulfonyldifluoroester for trifluoromethylthiolation of aryl iodides. ${ }^{[482]}$

$$
\text { MeCN, } 0.5 \mathrm{~h}, 50{ }^{\circ} \mathrm{C}
$$

Scheme 119. Synthesis of aryl trifluoromethyl thioethers from aryl diazonium salts with copper trifluoromethylthiolate

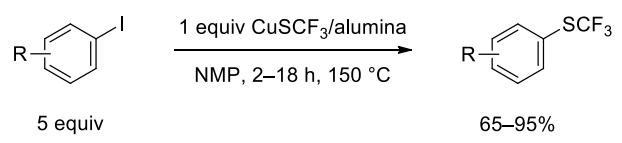

Scheme 120. Synthesis of aryl trifluoromethyl thioethers from aryl iodides and copper trifluoromethylthiolate

Palladium catalysis for the synthesis of aryl trifluoromethyl thioethers was developed by Buchwald with silver trifluoromethylthiolate nucleophile and aryl bromide electrophiles for the synthesis of a variety of aromatic and heteroaromatic trifluorothiolates (Scheme 121). ${ }^{[483]}$ A combination of a nickel catalyst with tetramethylammonium trifluoromethylthiolate was described by the Vicic group to convert aryl iodides to aryl trifluoromethylsulfide (Scheme 122). ${ }^{[44]}$ This method was later extended to boronic acid substrates using a copper catalyst. ${ }^{[485]}$ While nickel-catalyzed trifluoromethylthiolation of simple aryl iodides occurs at room temperature and does not necessitate silver trifluoromethylthiolate, the substrate scope is more limited than that displayed by the palladium-catalyzed reaction developed by the Buchwald group. Qing and co-workers established the crosscoupling of arylboronic acids with octathiocane (sulphur, $\mathrm{S}_{8}$ ) in the presence of $\mathrm{TMSCF}_{3}$, and two equivalents of silver carbonate catalyzed by copper(I) salts at room temperature to afford the desired products in $58-91 \%$ yield. ${ }^{[486]}$ Terminal alkynes were also shown to be viable substrates for this transformation.. ${ }^{[487]}$ Amidedirected $\mathrm{C}-\mathrm{H}$ functionalization of arenes with trifluorosulfenyl groups was recently accomplished by Daugulis via copper catalysis with trifluoromethyldisulfide (Scheme 123). ${ }^{[48]}$
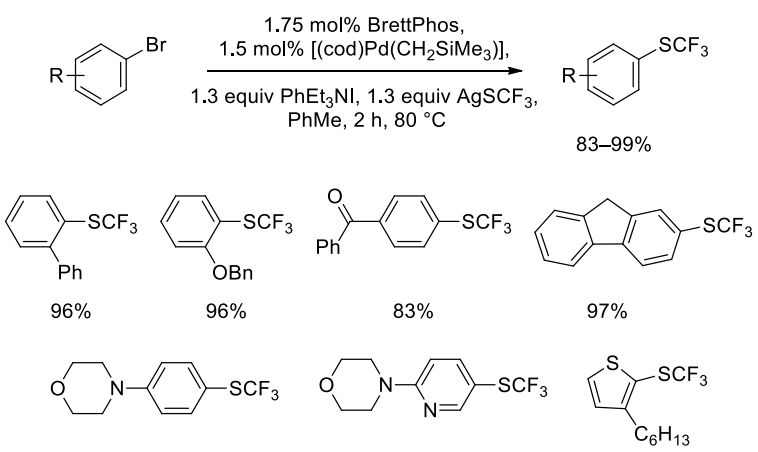<smiles>CC(C)(F)c1cnc2ccccc2c1</smiles>

$96 \%$

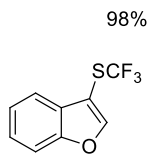

$81 \%$ $93 \%$

Scheme 121. Pd-catalyzed cross-coupling of aryl bromides with silver trifluoromethylthiolate activated with tetrasubstitued ammonium iodide

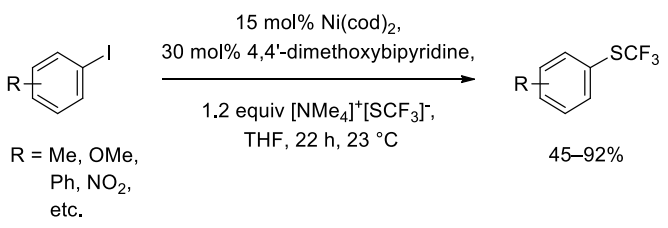

Scheme 122. Ni-catalyzed cross-coupling of aryl iodides with tetramethylammonium trifluoromethylthiolate

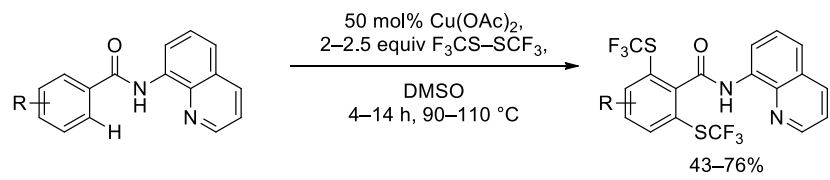

Scheme 123. Cu-catalyzed $\mathrm{N}$-directed $\mathrm{C}-\mathrm{H}$ functionalization of arenes with trifluoromethylsulfenyl groups

\subsection{Preparation of trifluoromethylamines}

Primary and secondary alkyl trifluoromethyl amines have been difficult to synthesize due to facile decomposition, mostly via elimination of fluoride, while tertiary alkyl trifluoromethyl amines can be synthesized but are difficult to isolate. ${ }^{[458]}$ Nitrogenous nucleophiles with less nucleophilic nitrogen lone pairs such as anilines, pyridine, and azoles can be successfully trifluoromethylated and isolated. ${ }^{[459,489]}$

The main approaches for the synthesis of trifluoromethylamines involve nucleophilic fluorination of thiocarbonyl-functionalized amines and $N$-trifluoromethylation of amines. Amines are first derivatized to thiuramide sulfides (Scheme 124) ${ }^{[107 c]}$ ), ${ }^{[107 c, 490]} \mathrm{N}$ alkyl dithiocarbamates (Scheme 125) ${ }^{[491]}$ or formamides ${ }^{[458,492]}$ followed by fluorination with nucleophilic fluoride sources. The formamide approach typically affords trifluoromethylamine products in high yield but involves the use of gaseous and toxic $\mathrm{SF}_{4}$. This functionalization-fluorination reaction sequence was also reported with $N$-perfluoroalkyl dithiocarbamates ${ }^{[493]}$ and $N$ heteroaryl dithiocarbamates (Scheme 126) ${ }^{[494]}$. 


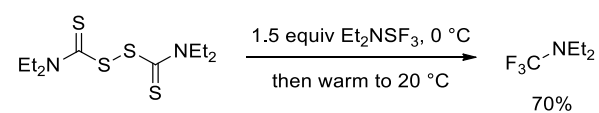

Scheme 124. Fluorodesulfurization of thiuramide sulfides

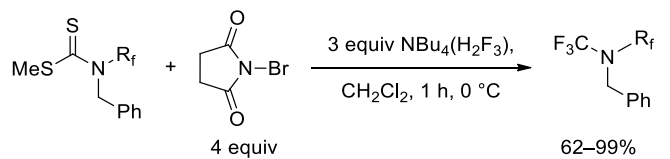

Scheme 125. Fluorodesulfurization of dithiocarbamates

$$
\begin{array}{ll}
\mathrm{R}=\mathrm{alkyl}, \mathrm{Bn} & 4 \text { equiv } \\
\mathrm{Y}=\mathrm{C} \text { or } \mathrm{N} &
\end{array}
$$

Scheme 126. Fluorodesulfurization of $N$-heteroaryl dithiocarbamates

Although $S$-(trifluoromethyl)dibenzothiophenium salts react with nitrogenous nucleophiles to afford $C$-trifluoromethylated products, ${ }^{[271 b]} O$-(trifluoromethyl)dibenzoxonium reagent $\mathbf{6 . 1}$ reacts with nitrogenous nucleophiles to afford $N$-trifluoromethylated anilines, primary, secondary and tertiary alkyl amines, and nitrogenous heteroarenes (Scheme 127). ${ }^{[459]}$ The $O$ (trifluoromethyl)dibenzofuranium reagent $\mathbf{6 . 1}$ by Umemoto is the same, thermally unstable, reagent used for the $O$ trifluoromethylation of phenols. $N$-Trifluoromethylation of nitriles after a Ritter-type reaction with azoles has been reported by Togni (Scheme 128). ${ }^{[495]}$ Subsequently, the same group reported the direct $N$-trifluoromethylation of azoles in $13-66 \%$ yield. ${ }^{[489]}$ Additionally, primary phosphines can undergo $P$-trifluoromethylation with Togni reagent II. ${ }^{[496]}$

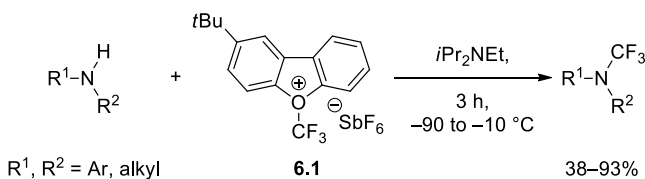

Scheme 127. Electrophilic N-trifluoromethylation of amines

$$
\begin{aligned}
& 1.5 \text { equiv } \\
& \begin{array}{l}
\mathrm{X}=\mathrm{C} \text { or } \mathrm{N} \\
\mathrm{R}=\text { alkyl, } \mathrm{Ar}
\end{array}
\end{aligned}
$$

Scheme 128. N-Trifluoromethylation of Ritter-type reaction products

\section{Fluorinated alkenes}

Fluoroalkenes find applications as peptide mimics ${ }^{[497]}$ and as monomers in polymerization reactions, e.g. for the synthesis of Teflon. ${ }^{[498]}$ Because the fluoroalkene moiety has a size and dipole moment similar to an amide bond while being difficult to hydrolyze ${ }^{[499]}$ molecules containing the fluoroalkene motif have been used as inhibitors of peptidases. ${ }^{[500]}$

\subsection{Preparation of monofluoroalkenes}

Monofluoroalkenes can be synthesized by Wittig olefination of carbonyls with triphenylphosphonium monofluoromethylide (Scheme 129), ${ }^{[501]}$ by Peterson-type olefination of aldehydes with $\alpha$ fluoro- $\alpha$-trialkylsilyl-esters with 50:1 Z/E stereoselectivity, ${ }^{[502]}$ and by Julia-Kocienski-modified conditions for the fluoromethylenation of ketones ${ }^{[503]}$ in up to $15: 1 \mathrm{E} / \mathrm{Z}$ stereoselectivity (Scheme 130). ${ }^{[503 \mathrm{a}]}$ $\alpha$-Fluorostyrenes can be synthesized from $\alpha, \alpha$-arylfluoromethyl 2 benzathiazolylsulfone reagents and paraformaldehyde in the presence of a base. ${ }^{[504]}$ Additionally, aldol condensation chemistry has been reported to introduce the monofluoromethyne group. ${ }^{[505]}$

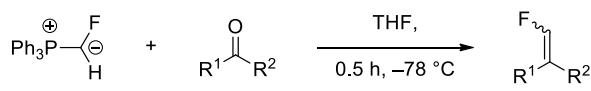

$$
\begin{aligned}
& \mathrm{R}^{1}, \mathrm{R}^{2}=\mathrm{H}, \mathrm{Ar} \text {, alkyl } \quad 16-65 \%
\end{aligned}
$$

Scheme 129. Synthesis of monofluoroalkenes via Wittig olefination of carbonyls

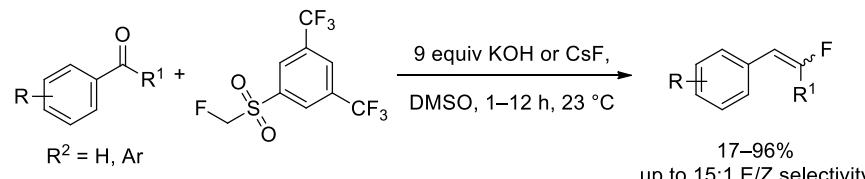

Scheme 130. Synthesis of monofluoroalkenes via Julia-Kocienski reaction with carbonyls

An addition-elimination sequence of fluorobis(phenylsulfonyl)methane with alkyl halides was used to afford monofluoroalkenes (Scheme 131). ${ }^{[194]}$ The selectivity for the $E$-isomer is proposed to arise from neighboring group participation by the aryl group during the elimination step. The stereoselective synthesis of $Z$-fluoroalkenes was achieved by addition of fluorosulfoximine $\mathbf{7 . 1}$ to nitrones followed by elimination of nitrosobenzene (Scheme 132) ${ }^{[506]}$ In the proposed transition-state, the two aryl groups are positioned gauche for the formation of the disfavored $E$-isomer; therefore, a preference for the $Z$-isomers would be predicted.

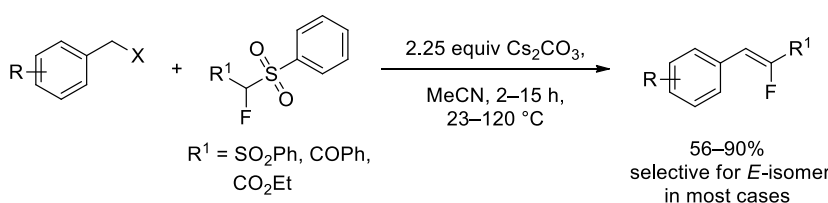

Scheme 131. Synthesis of monofluoroalkenes via Julia-Kocienskitype reaction with benzyl halides 

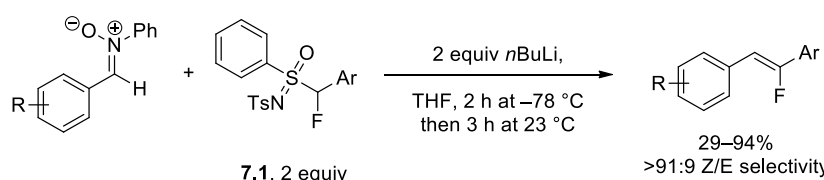

Scheme 132. Synthesis of monofluoroalkenes via nitrone addition with fluoromethyl pronucleophile

Monofluoroalkene synthesis via alkyne hydrofluorination was first accomplished with polymer-supported dihydrogen trifluoride ${ }^{[507]}$ and tetrabutylammonium dihydrogen trifluoride. ${ }^{[508]}$ Transitionmetal-mediated hydrofluorination was demonstrated by Sadighi via reversible gold-mediated hydrofluorination of substituted alkynes with triethylamine-hydrogen fluoride (Scheme 133). ${ }^{[509]}$ Subsequently, gold-catalyzed $N$-directed synthesis of fluoroalkenes from disubstituted alkynes and triethylamine-hydrogen fluoride was shown to yield $>50: 1$ regioselectivity by Miller and co-workers (Scheme 134). ${ }^{[510]}$ The reaction can also be ester-directed but regioselectivity is lower; 1:1.5 in favor of isomer B in some cases (see Scheme 134).
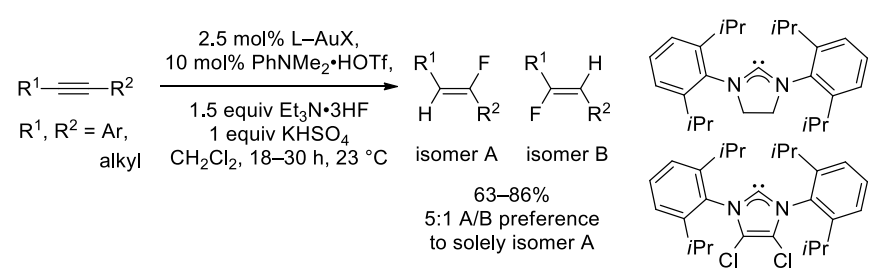

Scheme 133. Au-catalyzed hydrofluorination of alkynes

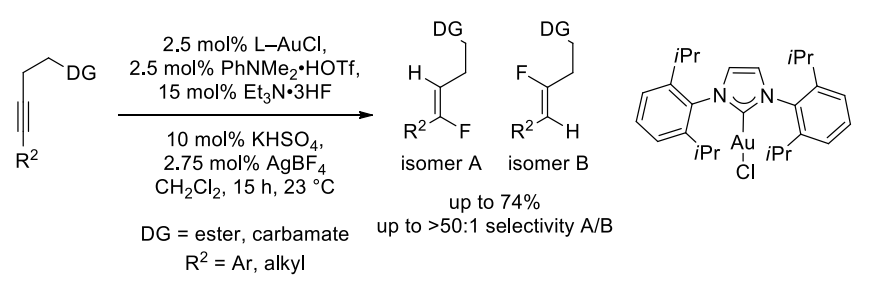

Scheme 134. Au-catalyzed ester-directed hydrofluorination of alkynes

Silver-mediated electrophilic fluorodestannylation of alkenyl stannanes reported by Tius demonstrated that the synthesis of fluoroalkenes could be accomplished via electrophilic fluorination. ${ }^{[34 i, 46]}$ Gouverneur reported the gold-catalyzed cyclization-fluorination of propargyl ketones with F-TEDA-BF 4 to afford cyclic $\alpha$-fluoro vinylogous esters (Scheme 135) ${ }^{[511]}$ with the protodeaurated product accounting for most of the byproduct. Intermediates of gold-carbene-catalyzed rearranged proparyl acetates can also be fluorinated electrophilically to afford $\alpha$ fluoroenones as describe by Nevado (Scheme 136) ${ }^{[512]}$ Only alkylor phenyl- functionalized propargyl acetates were reported to undergo this rearrangement.

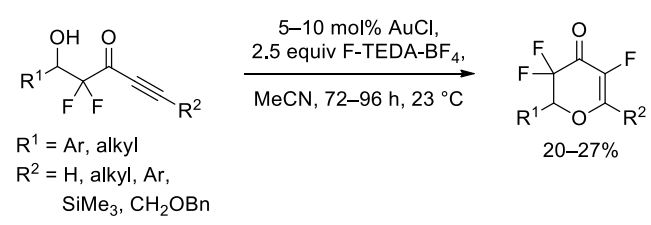

Scheme 135. Au-catalyzed fluorocyclization of propargyl ketones

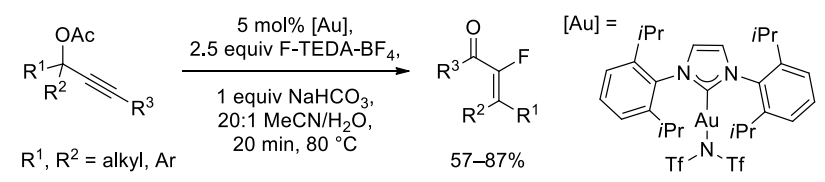

Scheme 136. Synthesis of $\alpha$-fluoroenones via Au-catalyzed 1,3acyloxy rearrangement of propargyl acetates

Nucleophilic $\mathrm{S}_{\mathrm{N}} 2$ ' additions of 3,3-difluoropropenes with cuprates ${ }^{[513]}$ organolithium reagents, ${ }^{[514]}$ and amines ${ }^{[515]}$ by Paquin and co-workers have been used to synthesize double-bond transposed fluorinated allylic products (Scheme 137). In contrast to highly nucleophilic organolithiums, neutral amine nucleophiles required the use of palladium complexes to first activate 3,3difluoropropenes as an allyl fragment. With organolithium reagents monoiodoalkenes are lithiated and fluorinated with $N$-fluoro- $N$-tertbutylphenylsulfonamide affording the same double bond geometry ${ }^{[516]}$ trifluoromethyl tosylhydrazones, on the other hand, rearrange to monofluoroalkenes with an excess of alkyl lithium reagents. ${ }^{[517]}$

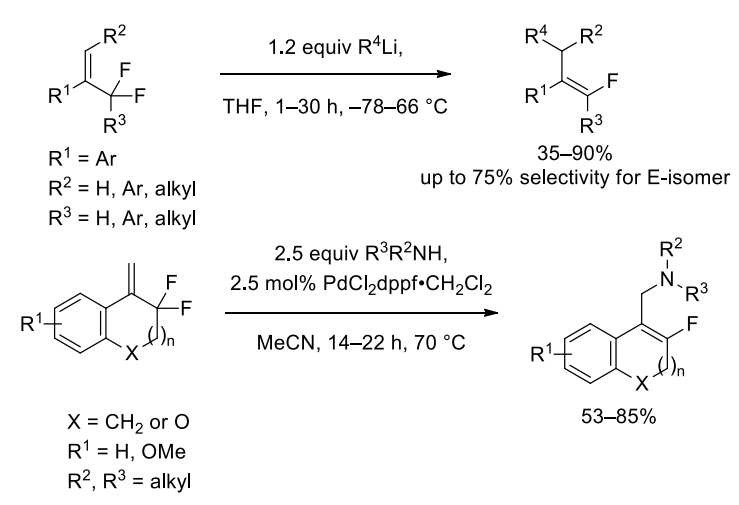

Scheme 137. Alkyl lithium addition and Pd-catalyzed allylic alkylation of amines of 3,3-difluoropropenes

Palladium-catalyzed cross-coupling of terminal alkynes with iodofluoroalkenes affords fluorinated enyne products, ${ }^{[518]}$ while fluorovinyl tosylate undergoes cross-coupling with boronic acids to yield terminal fluoroalkene. ${ }^{[519]}$ Copper-catalyzed reaction of Freon113 (1,1,1-difluorochloro-2,2,2-dichlorofluoroethane) with aryl hydrazones produces fluorinated alkene derivatives with release of $\mathrm{N}_{2}{ }^{[520]}$

\subsection{Preparation of $\alpha, \alpha$-difluoroalkenes and 1,2- difluoroalkenes}

\subsubsection{Preparation of $\alpha$, $\alpha$-difluoroalkenes}


Approaches used to synthesize difluoroalkenes are similar to those of monofluoroalkenes which include Wittig and (Scheme $138)^{[501 b, 501 c, 521]}$ Horner-Wadsworth-Emmons olefination ${ }^{[522]}$ as well as difluoromethylenation with bis(trifluoromethyl) mercury, ${ }^{[523]}$ copper and difluorodibromomethane, ${ }^{[524]}$ and difluorophenylsulfenyltrimethylsilane. ${ }^{[525]}$ Generally, carbonyl difluoromethylenation reactions with difluoromethylenating reagents give rise to yields below $70 \%$. The addition-elimination sequence used to synthesize monofluoroalkenes from alkyl halides and fluoromethyl phenyl sulfone ${ }^{[526]}$ has adapted to the synthesis of difluoroalkenes through the use of difluoromethyl phenyl sulfone and alkyl halides (Scheme 139). ${ }^{[526 b]}$ Although the method by Prakash shown in Scheme 139 utilizes a slight excess of starting material to base, it provides access to aliphatic and aryl difluoroolefins in a synthetically practical manner.

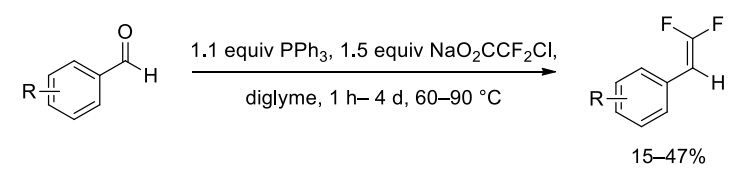

Scheme 138. Synthesis of difluoroalkenes via Wittig-type olefination of aldehydes

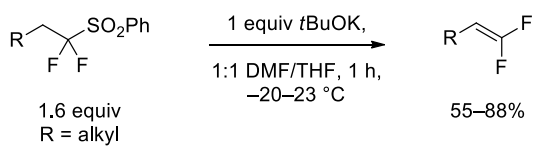

Scheme 139. Elimination of fluoromethyl alkylated products to afford difluoroalkenes

Geminally-substituted trifluoromethyl dimethylphenylsilyl alkenes can be transformed to the 1,1-difluoroalkenes via $\mathrm{S}_{\mathrm{N}} 2$ ' addition of various nucleophiles like LAH, organolithium reagents, and lithium enolates (Scheme 140). ${ }^{[527]}$ Similarly, trifluoromethyl styrenes can rearrange to geminal difluoroalkenyl amines ${ }^{[528]}$ after addition of lithium amide or allylic alkyl difluoroalkenes ${ }^{[529]}$ or organolithium reagents (Scheme 140). More recently, the addition of substituted hydrazine nucleophiles to trifluoromethyl styrene followed by intramolecular cyclization was shown to yield various 3-fluoropyrazole products. ${ }^{[530]}$ Trifluoromethyl tosylhydrazones rearrange to difluoroalkenes with 2.5 equivalents of $n \mathrm{BuLi}$ or methyl lithium through extrusion of $\mathrm{N}_{2}$ gas and tolylsulfinate (Scheme 141). ${ }^{[517]}$ Similarly, $n \mathrm{BuLi}$ induces opening of chlorodifluoromethyl epoxides to afford 1,1-difluoroallylic alcohols. $^{[531]}$

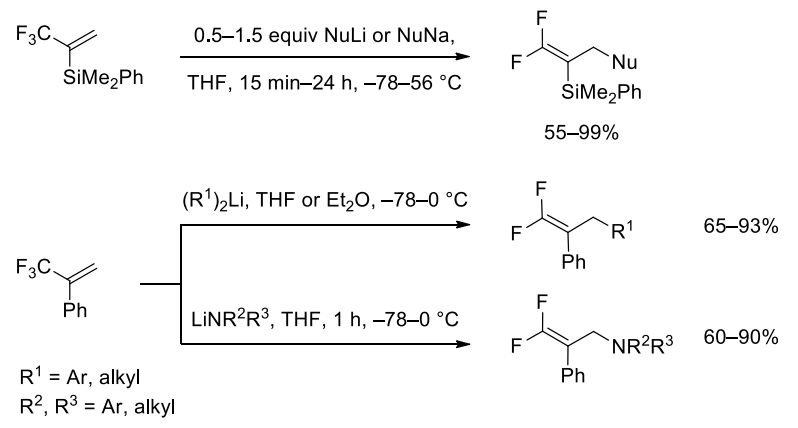

Scheme 140. $\mathrm{S}_{\mathrm{N}} 2^{\prime}$ addition of various nucleophiles to $3,3,3-$ trifluoropropene

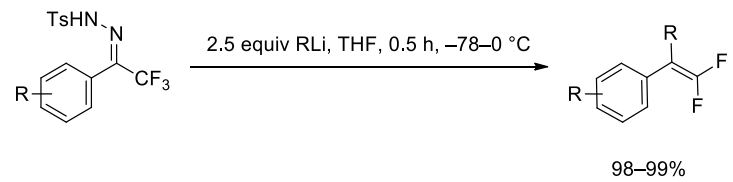

Scheme 141. Alkyl lithium-induced rearrangement of aryl trifluoromethyl tosylhydrazones to terminal 1,1-difluorostyrenes

Cross-coupling methods provide an expedient route for the installation of difluoroalkenyl groups from commercially available arene precursors. Trifluoromethyl groups of $\alpha$ trifluoromethylstyrenes undergo $\beta$-fluoride elimination in the presence of rhodium catalysts and then participate in a crosscoupling reaction with arylboronic acids to give 1,1-difluoroallylic aryl products (Scheme 142). ${ }^{[532]}$ Cross-coupling of difluoroalkenylzinc reagents with aryl iodides under palladium catalysis yields difluorostyrenyl products, ${ }^{[533]}$ while difluoroenol derivatives such as $\alpha, \alpha$-difluoroketene iodoacetals can be crosscoupled with stannanes or boronic acids to afford aryl difluoroenol products. ${ }^{[534]}$

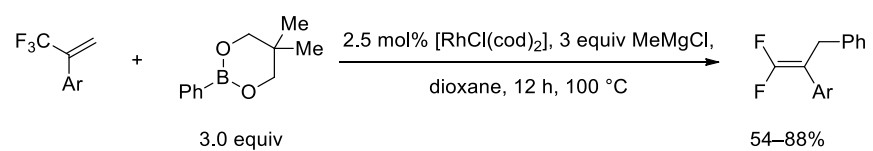

Scheme 142. Rh-catalyzed coupling of 3,3,3-trifluoropropene with arylboronic ester with $\beta$-fluoride elimination

\subsubsection{Preparation of 1,2-difluoroalkenes}

1,2-Difluoroalkenes can be synthesized in up to $97 \%$ yield by elimination of hydrogen bromide from 1,1,1-dibromofluoro-2fluorosubstituted substrates with up to $99: 1 \mathrm{E} / \mathrm{Z}$ selectivity ${ }^{[535]}$ as well as by protodesilyation of 1,2-difluoro-1-silylalkene. ${ }^{[536]} 1,2$ Difluoroolefins can additionally be synthesized via cross-coupling of the fluorinated alkenyl zinc reagent with aryl iodides (Scheme 143) ${ }^{[537]}$ allowing for the late-stage introduction of vicinal difluoroalkenyl groups into arenes. Treatment of $\alpha$-keto carbonyls with Deoxo-Fluor ${ }^{\circledR}$ yields $\alpha, \beta$-difluoroenone (Scheme 144). ${ }^{[538]}$

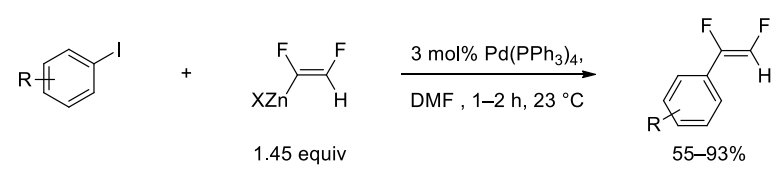

Scheme 143. Pd-catalyzed cross-coupling of aryl iodides with 1,2difluoroalkenyl zinc reagent

$$
\begin{aligned}
& \underset{\mathrm{CH}_{2} \mathrm{Cl}_{2}, 15 \mathrm{~h}, 35^{\circ} \mathrm{C}}{\stackrel{3 \text { equiv Deox-Fluor }}{\circledR},} \\
& R^{1}, R^{2}=\text { Ar, alkyl } \quad \text { up to } 80 \%
\end{aligned}
$$

Scheme 144. Synthesis of $\alpha, \beta$-difluoroenones via nucleophilic fluorination of Deoxo-Fluor ${ }^{\circledR}$ 


\section{Fluorination with fluorine-18 for positron emission tomography (PET)}

Positron emission tomography (PET) is widely recognized as a clinical tool for cancer diagnosis, with other applications emerging in medical research and patient care. ${ }^{[5 \mathrm{c}, \mathrm{d}]}$ PET relies on the use of radiotracers that bear a radionuclide which decays via positron emission, such as fluorine-18. Simple radiotracers containing fluorine-18 can be synthesized via conventional fluorinations methods. But conventional fluorination methods typically have low functional group tolerance and cannot afford complex, biomedically relevant molecules as PET tracers. Due to the short half-life of 110 minutes of fluorine-18, synthesis of radiotracers requires that fluorine-18 introduction occur at a late-stage of the synthesis to avoid unproductive decay. The time used to synthesize, purify, and formulate radiotracers for injection should be less than two halflives of the radionuclide. The ideal method for fluorine-18 introduction is functional group tolerant, fast, shows low watersensitivity, affords a single fluorinated product, and requires only straightforward purification. We will only cover recent, conceptually novel approaches for $\mathrm{C}-{ }^{18} \mathrm{~F}$ bond formation; comprehensive reviews are available. \{Miller, 2008 \#855;Cai, 2008 \#1032;Ametamey, 2008 \#748;Phelps, 2000 \#858;Bolton, 2002 \#752;Tredwell, 2012 \#2848)

\subsection{Nucleophilic methods for the incorporation of ${ }^{18} F$}

Fluorine-18 is synthesized through bombardment of oxygen-18enriched water with protons to afford aqueous ${ }^{18} \mathrm{~F}$-fluoride in high specific activity. Desolvation of aqueous ${ }^{18} \mathrm{~F}$-fluoride to afford anhydrous ${ }^{18} \mathrm{~F}$-fluoride is difficult due to the strong hydrogen bonding interaction of fluoride anions with water.\{Emsley, 1980 \#2226\} ${ }^{18} \mathrm{~F}$-enriched fluorine gas is generally of lower specific activity than ${ }^{18} \mathrm{~F}$-fluoride, but recent advances have increased the specific activity obtainable for $\left[{ }^{18} \mathrm{~F}\right] \mathrm{F}_{2} \cdot .{ }^{[539]}{ }^{18} \mathrm{~F}$-Fluoride is the most practical and most widely available source of ${ }^{18} \mathrm{~F}$.

Radiofluorination reactions with ${ }^{18} \mathrm{~F}$-fluoride most commonly consist in substitution reactions that occur on alkyl or aryl electrophiles, functionalized with appropriate leaving groups. Alkali salts are typically used along with cryptands to increase fluoride nucleophilicity. ${ }^{[5 \mathrm{~d}]}$ Reactions solvents are chosen such that $\mathrm{S}_{\mathrm{N}}$ 2-type and $\mathrm{S}_{\mathrm{N}}$ Ar-type reactions are facilitated, with the most commonly used solvents being polar aprotic solvents such as DMF, DMSO, and acetonitrile. Tert-butanol may be used to temper the basicity of ${ }^{18} \mathrm{~F}$-fluoride for aliphatic substitution reactions. ${ }^{[105]}$ The most commonly used radiotracer for imaging, 2-deoxy-2-[ $\left.{ }^{18} \mathrm{~F}\right]$ fluoro-Dglucose $\left(\left[{ }^{18} \mathrm{~F}\right] \mathrm{FDG}\right)$, is made via nucleophilic substitution of a triflate leaving group on a mannose triflate derivative with ${ }^{18} \mathrm{~F}$ fluoride (Scheme 145). ${ }^{[540]}$ For the synthesis of ${ }^{18} \mathrm{~F}$-functionalized allylic fluorides, Gouverneur and co-workers reported the first palladium-catalyzed fluorination reaction with ${ }^{18} \mathrm{~F}$-fluoride. ${ }^{[167]}$

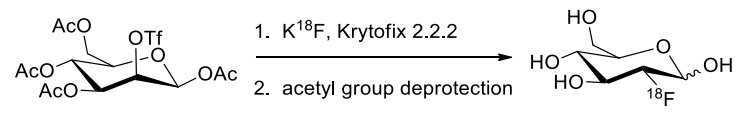

Scheme 145. Nucleophilic radiochemical fluorination with ${ }^{18} \mathrm{~F}$-fluoride to afford $\left[{ }^{18}\right.$ F $F$ FDG

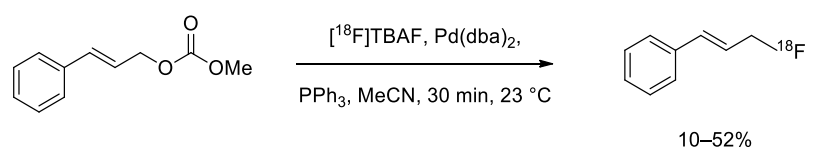

radiochemical yield $(\mathrm{RCY})$

Scheme 146. Pd-catalyzed allylic fluorination with ${ }^{18} \mathrm{~F}$-fluoride

Nucleophilic aromatic substitution with ${ }^{18} \mathrm{~F}$-fluoride can be used to synthesize ${ }^{18} \mathrm{~F}$-labelled arenes. ${ }^{[541]}$ For substrates to undergo $\mathrm{S}_{\mathrm{N}} \mathrm{Ar}$-type substitution reactions, they generally require at least one electron-withdrawing group on the arene ortho or para to the leaving group, which can be a nitro, trialkylammonium, halide, or sulfonate groups. Nicotinic acetylcholine receptor radioligands have been synthesized via nucleophilic aromatic substitution (Scheme 147). ${ }^{[542]}$

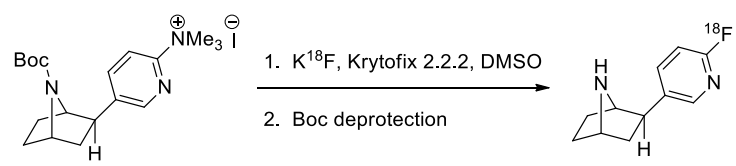

Scheme 147. Nucleophilic aromatic substition with ${ }^{18} \mathrm{~F}$-fluoride

Diaryliodonium salts 8.1 can be fluorinated with ${ }^{18} \mathrm{~F}$-fluoride to yield radiolabelled aryl fluorides. ${ }^{[543]}$ The selectivity for fluorination between the two arenes attached to iodine is based on the electronic structure of the arenes (the more electron-poor arene is fluorinated) and the steric bulk near iodine (ortho-substituted arenes are more susceptible to fluorination due the ortho-effect ${ }^{[544]}$ ) (Scheme 148). Electron rich 2-thiophene can be used to advantage as a dummy ligand on iodine. ${ }^{[543 b]}$ The counterion of the iodonium salts also have a large effect on the yield of fluorination, with the more dissociative and non-nucleophilic counteranions promoting higher radiochemical yields. With ortho-substituted substrates, the radiochemical yields can be as high as $\sim 60 \%$. The availability of more straightforward syntheses of complex diaryliodonium salts would increase the utility of the method and its use for PET tracer synthesis. Current syntheses of aryl iodonium salts often employ strong Lewis or Brønsted acids. ${ }^{[545]}$

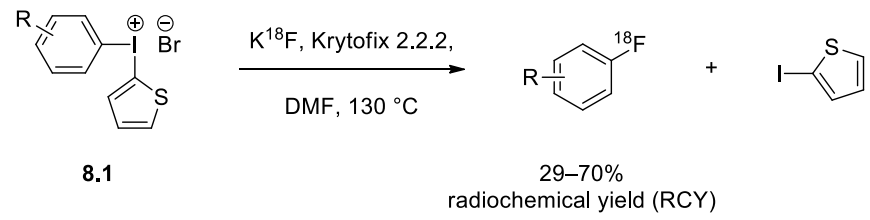

Scheme 148. Nucleophilic radiochemical fluorination of aryl(2thienyl)iodonium bromides

\subsection{Electrophilic methods for the incorporation of ${ }^{18} F$}

Several ${ }^{18}$ F-electrophilic fluorinating reagents have been synthesized from ${ }^{18} \mathrm{~F}$-fluorine gas such as ${ }^{18} \mathrm{~F}$-acetyl hypofluorite, ${ }^{[546]}{ }^{18} \mathrm{~F}$-xenon difluoride, ${ }^{[546 a,}{ }^{547]}{ }^{18} \mathrm{~F}-\mathrm{N}$ fluorosulfonamide or imide reagents, ${ }^{[548]}{ }^{18} \mathrm{~F}-\mathrm{N}$-fluoropyridinium salts, ${ }^{[549]}$ and ${ }^{18}$ F-TEDA salts. ${ }^{[539 b]}$ Electrophilic radiofluorination via direct, non-specific fluorination of substrates such as alkenes ${ }^{[550]}$ for the synthesis of $\left[{ }^{18} \mathrm{~F}\right] \mathrm{FDG}$ and related sugars as well as arenes ${ }^{[551]}$ for the synthesis of $\left[{ }^{18} \mathrm{~F}\right]$ fluoro-3,4-dihydroxy phenylalanine $\left(\left[{ }^{18} \mathrm{~F}\right] \mathrm{F}-\mathrm{DOPA}\right)$ can lead to multiple fluorinated 
products and typically are not functional group tolerant. Radiochemical fluorodemetalation has been used for aryl organometallic reagents, ${ }^{[548 \mathrm{a}]}$ aryl silanes, ${ }^{[37 \mathrm{c}, \mathrm{d}]}$ and aryl stannanes ${ }^{[34 \mathrm{a},}$ $34 c, 546 b]$ and can afford selective fluorination.

Gouverneur developed the oxidative fluorination of para-tertbutylphenols with replacement of the para-tert-butyl group with ${ }^{18} \mathrm{~F}$ fluoride in the presence of iodobenzene diacetate and trifluoroacetic acid in dichloromethane (Scheme 149). ${ }^{[552]}$ The reaction is proposed to occur via oxidative fluorination/dearomatization followed by rearomatization. The radiochemical yields for various para-tertbutylphenol derivatives range from $7-21 \%$ and the reaction tolerates a wide range of electronically-diverse ortho-substituents including halides, other tert-butyl groups, carbonyls, and olefins.
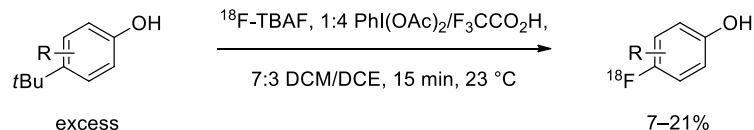

$$
7-21 \%
$$
radiochemical yield $(\mathrm{RCY})$

$$
\text { }
$$

$$
{ }_{18} \overbrace{\mathrm{Fe}}^{\mathrm{OH}}
$$

$$
{ }_{18}^{18}
$$$$
16 \% \text { RCY }
$$$$
21 \% \operatorname{RCY}
$$<smiles>O=C(c1ccccc1)c1cc(F)ccc1O</smiles><smiles>Oc1ccc(F)cc1Br</smiles><smiles>Cc1cc(O)ccc1F</smiles>

$7 \% \mathrm{RCY}$

$18 \% \operatorname{RCY}$

$16 \%$ RCY

Scheme 149. Oxidative radiochemical fluorination of para-tert-butyl phenols with ${ }^{18} \mathrm{~F}$-fluoride and iodobenzene diacetate

An electrophilic radiofluorination for the synthesis of aryl fluorides in high specific activity utilizes $\operatorname{Pd}(\mathrm{IV})$ complex $\mathbf{8 . 2}$ to incorporate ${ }^{18} \mathrm{~F}$-fluoride into complex arenes (Scheme 150). ${ }^{[553]}$ The $\mathrm{Pd}(\mathrm{IV})$ complex captures fluoride and then functions as electrophilic $\left[{ }^{18} \mathrm{~F}\right]$ fluorination reagent. Direct $\mathrm{S}_{\mathrm{N}} 2$ transfer and an electroncoupled fluoride transfer have both been proposed as possible reaction pathways. An advantage of using this method is that ${ }^{18} \mathrm{~F}$ fluoride can be incorporated into functionalized arenes at a late stage. Additionally, the $\mathrm{Pd}(\mathrm{IV})-\left[{ }^{18} \mathrm{~F}\right]$ complex is thermally stable and insensitive to water.

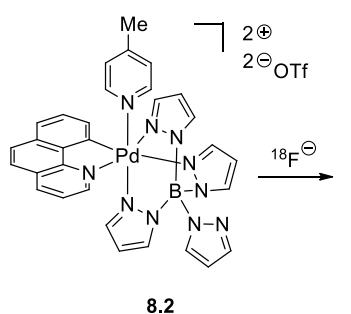

8.2

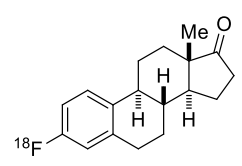

$33 \% \pm 7 \% \operatorname{RCY}(n=8)$

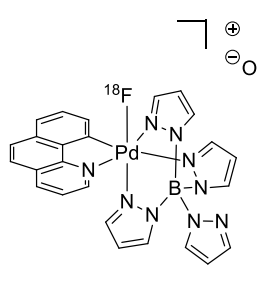
$\Theta_{\text {OTf }}$
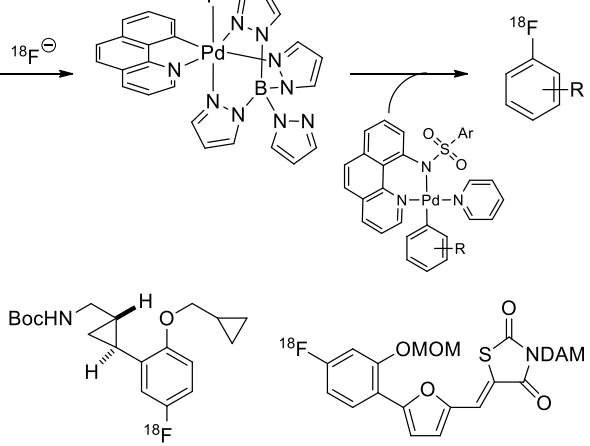

$18 \% \pm 5 \% \operatorname{RCY}(n=8)$

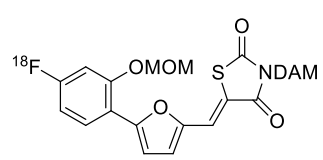

$10 \% \pm 2 \% \operatorname{RCY}(n=7)$
Scheme 150. Synthesis of an electrophilic ${ }^{18} \mathrm{~F}-\mathrm{Pd}(\mathrm{IV})$ fluorinating reagent from ${ }^{18} \mathrm{~F}$-fluoride to afford e for the synthesis of ${ }^{18} \mathrm{~F}$-labelled aryl fluorides
$\mathrm{Ni}(\mathrm{II})$ aryl pyridylsulfonamide complexes can be oxidized with oxidant 8.3 in the presence of aqueous ${ }^{18} \mathrm{~F}$-fluoride to afford complex ${ }^{18}$ F-labelled arenes in 13-58\% RCY (Scheme 151). ${ }^{[554]}$ The use of aqueous fluoride-18 solution obviates the need for time consuming anion exchange and azeotropic drying steps during PET tracer synthesis. Radiofluorination takes place at room temperature and is complete within less than one minute.
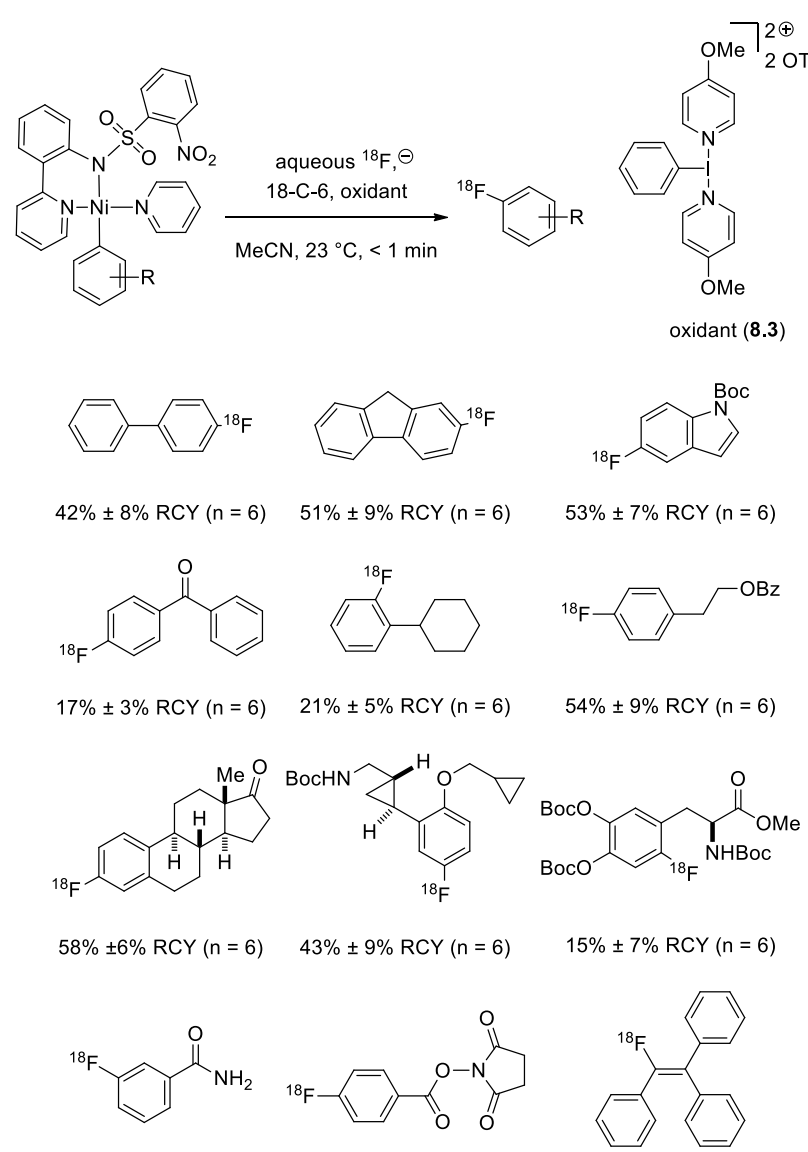

$38 \% \pm 7 \% \operatorname{RCY}(n=6) \quad 21 \% \pm 4 \% \operatorname{RCY}(n=6)$

$13 \% \pm 3 \% \operatorname{RCY}(n=6)$

Scheme 151. Synthesis of ${ }^{18} \mathrm{~F}$-labelled aryl fluorides from $\mathrm{Ni}(\mathrm{II})$ complexes, oxidant, and aqueous ${ }^{18} \mathrm{~F}$-fluoride

\section{Outlook}

The most significant, conceptual advances in the past decade of fluorination chemistry, broadly defined, were made in the areas of carbon-fluorine and carbon- $\mathrm{CF}_{3}$ bond formation reactions, most prominently via organo- and transition-metal catalysis. The most challenging transformation remains the parent $\mathrm{C}-\mathrm{F}$ bond formation, primarily due to the high hydration energy of fluoride, strong metalfluorine bonds, and the highly polarized nature of bonds to fluorine.

Fluorination chemistry still lacks general predictability and practicality. For example, commonly employed electrophilic fluorinating and trifluoromethylating reagents are often not cost efficient on manufacturing scale. Even nucleophilic methods often require expensive reagents or catalysts, which reduce the practicality of modern fluorination reactions for large-scale synthesis. Despite these limitations, modern methods for fluorination chemistry have made fluorinated molecules more readily available than ever before. In particular, the modern methods start to impact research areas that 
do not require large amounts of material, such as drug discovery and PET. Future research in the field should focus on the development of practical and selective fluorination reactions, with readily available starting materials and cost-efficient reagents. The ideal fluorination reaction would be predictable, general, and functional group tolerant, use fluoride, and be catalyzed by a readily available, inexpensive catalyst.

The authors thank Dr. Jean Raynaud, Anthony R. Mazzotti, Dr. Jochen R. Brandt, Dr. Ming-Yu Ngai, and Dr. Takeru Furuya for helpful discussions.

Received: ((will be filled in by the editorial staff))

Published online on ((will be filled in by the editorial staff))

a) A. Borodine, Ann. Chem. Pharm. 1863, 126, 58-62; b) O. Wallach, Justus Liebigs Ann. Chem. 1886, 235, 233-255; c) F. Swarts, Bull. Acad. R. Med. Belg. 1898, 35, 375-420.

a) H. J. Bohm, D. Banner, S. Bendels, M. Kansy, B. Kuhn, K. Muller, U. Obst-Sander, M. Stahl, ChemBioChem 2004, 5, $637-$ 643; b) K. L. Kirk, Curr. Top. Med. Chem. 2006, 6, 1445-1445; c) K. L. Kirk, Curr. Top. Med. Chem. 2006, 6, 1447-1456; d) K. Muller, C. Faeh, F. Diederich, Science 2007, 317, 1881-1886; e) W. K. Hagmann, J. Med. Chem. 2008, 51, 4359-4369; f) D. O'Hagan, Chem. Soc. Rev. 2008, 37, 308-319; g) S. Purser, P. R. Moore, S. Swallow, V. Gouverneur, Chem. Soc. Rev, 2008, 37 , 320-330; h) J.-P. Begue, D. Bonnet-Delpon, J. Fluorine Chem. 2006, 127, 992-1012; i) F. M. D. Ismail, J. Fluorine Chem. 2002, $118,27-33$; j) C. Isanbor, D. O'Hagan, J. Fluorine Chem. 2006, 127, 303-319.

[3] a) P. Jeschke, ChemBioChem 2004, 5, 570-589; b) P. Maienfisch, R. G. Hall, Chimia 2004, 58, 93-99.

[4] a) F. Guittard, E. T. de Givenchy, S. Geribaldi, A. Cambon, $J$ Fluorine Chem. 1999, 100, 85-96; b) F. Babudri, G. M. Farinola, F. Naso, R. Ragni, Chem. Commun. 2007, 1003-1022; c) M. Cametti, B. Crousse, P. Metrangolo, R. Milani, G. Resnati, Chem. Soc. Rev. 2012, 41, 31-42; d) R. Berger, G. Resnati, P. Metrangolo, E. Weber, J. Hulliger, Chem. Soc. Rev. 2011, 40, 3496-3508; e) C. M. Kassis, J. K. Steehler, D. E. Betts, Z. B. Guan, T. J. Romack, J. M. DeSimone, R. W. Linton, Macromolecules 1996, 29, 3247-3254; f) M. G. Dhara, S. Banerjee, Prog. Polym. Sci. 2010, 35, 1022-1077; g) S. Schloegl, R. Kramer, D. Lenko, H. Schroettner, R. Schaller, A. Holzner, W. Kern, Eur. Polym. J. 2011, 47, 2321-2330; h) D. Anton, Adv. Mater. 1998, 10, 1197-1205; i) H. Saito, E. Nakagawa, T. Matsushita, F. Takeshita, Y. Kubo, S. Matsui, K. Miyazawa, Y. Goto, Ieice Transactions on Electronics 1996, E79C, 1027-1034; j) P. Kirsch, A. Hahn, Eur. J. Org. Chem. 2005, 3095-3100; k) Y. Li, Acc. Chem. Res. 2012, 45, 723-733.

[5] a) M. E. Phelps, Proc. Natl. Acad. Sci. USA 2000, 97, 9226-9233; b) R. Bolton, J. Labelled Compd. Radiopharm. 2002, 45, 485528 ; c) S. M. Ametamey, M. Honer, P. A. Schubiger, Chem. Rev. 2008, 108, 1501-1516; d) L. Cai, S. Lu, V. W. Pike, Eur. J. Org. Chem. 2008, 2853-2873; e) P. W. Miller, N. J. Long, R. Vilar, A. D. Gee, Angew. Chem. Int. Ed. 2008, 47, 8998-9033; f) R. Littich, P. J. H. Scott, Angew. Chem. Int. Ed. 2012, 51, 1106-1109.

[6] J. Emsley, Chem. Soc. Rev. 1980, 9, 91-124.
[7] a) F. C. Kupper, N. Schweigert, E. A. Gall, J. M. Legendre, H. Vilter, B. Kloareg, Planta 1998, 207, 163-171; b) C. Slebodnick, B. J. Hamstra, V. L. Pecoraro in Metal Sites in Proteins and Models: Phosphatases, Lewis Acids and Vanadium, Vol. 89 (Eds.: H. A. O. Hill, P. J. Sadler, A. J. Thomson), 1997, p. 51-108; c) A. S. Eustaquio, F. Pojer, J. P. Noe, B. S. Moore, Nature Chemical Biology 2008, 4, 69-74.

[8] G. W. Gribble, Acc. Chem. Res. 1998, 31, 141-152.

[9] D. B. Harper, D. O'Hagan, Natural Product Reports 1994, 11, 123-133.

[10] a) M. Sanada, T. Miyano, S. Iwadare, J. M. Williamson, B. H. Arison, J. L. Smith, A. W. Douglas, J. M. Liesch, E. Inamine, J. Antibiot. 1986, 39, 259-265; b) K. A. Reid, J. T. G. Hamilton, R.
D. Bowden, D. O'Hagan, L. Dasaradhi, M. R. Amin, D. B. Harper Microbiology-UK 1995, 141, 1385-1393; c) C. Schaffrath, S. L. Cobb, D. O'Hagan, Angew. Chem. Int. Ed. 2002, 41, 3913-3915; d) C. D. Murphy, C. Schaffrath, D. O'Hagan, Chemosphere 2003, 52, 455-461; e) C. J. Dong, F. L. Huang, H. Deng, C. Schaffrath, J. B. Spencer, D. O'Hagan, J. H. Naismith, Nature 2004, 427, 561-565.

[11] R. E. Banks, S. N. Mohialdinkhaffaf, G. S. Lal, I. Sharif, R. G Syvret, J. Chem. Soc., Chem. Commun. 1992, 595-596.

[12] a) W. J. Middleton, J. Org. Chem. 1975, 40, 574-578; b) W. J. Middleton, E. M. Bingham, Org. Synth. 1988, 50, 440-441.

[13] a) M. R. C. Gerstenberger, A. Haas, Angew. Chem. Int. Ed. 1981, 20,647-667; b) J. T. Welch, Tetrahedron 1987, 43, 3123-3197; c) M. A. McClinton, D. A. McClinton, Tetrahedron 1992, 48, 65556666; d) J. A. Wilkinson, Chem. Rev. 1992, 92, 505-519; e) M. A. Tius, Tetrahedron 1995, 51, 6605-6634; f) W. R. Dolbier, Chem. Rev. 1996, 96, 1557-1584; g) D. L. S. Brahms, W. P. Dailey, Chem. Rev. 1996, 96, 1585-1632; h) D. J. Burton, Z. Y. Yang, W. M. Qiu, Chem. Rev. 1996, 96, 1641-1715; i) T. Umemoto, Chem. Rev. 1996, 96, 1757-1777; j) J. A. Ma, D. Cahard, Chem. Rev. 2004, 104, 6119-6146; k) J.-A. Ma, D. Cahard, Chem. Rev. 2008, 108, PR1-43; 1) R. P. Singh, J. M. Shreeve, Acc. Chem. Res. 2004, $37,31-44$.

[14] Z. Jin, G. B. Hammond, B. Xu, Aldrichimica Acta 2012, 43, 67 83 .

[15] B. E. Smart, J. Fluorine Chem. 2001, 109, 3-11.

[16] a) M. B. van Niel, I. Collins, M. S. Beer, H. B. Broughton, S. K. F. Cheng, S. C. Goodacre, A. Heald, K. L. Locker, A. M. MacLeod, D. Morrison, C. R. Moyes, D. O'Connor, A. Pike, M. Rowley, M. G. N. Russell, B. Sohal, J. A. Stanton, S. Thomas, H. Verrier, A. P. Watt, J. L. Castro, J. Med. Chem. 1999, 42, 20872104; b) M. Morgenthaler, E. Schweizer, A. Hoffmann-Roeder, F. Benini, R. E. Martin, G. Jaeschke, B. Wagner, H. Fischer, S. Bendels, D. Zimmerli, J. Schneider, F. Diederich, M. Kansy, K. Mueller, ChemMedChem 2007, 2, 1100-1115.

[17] a) A. Leo, C. Hansch, D. Elkins, Chem. Rev. 1971, 71, 525-616; b) C. Hansch, A. Leo, S. H. Unger, K. H. Kim, Nikaitan.D, E. J. Lien, J. Med. Chem. 1973, 16, 1207-1216; c) C. Hansch, S. D. Rockwell, P. Y. C. Jow, A. Leo, E. E. Steller, J. Med. Chem. 1977, 20, 304-306; d) C. Hansch, A. Leo, R. W. Taft, Chem. Rev. 1991, 91, 165-195.

[18] A. Bondi, J. Phys. Chem. 1964, 68, 441-451.

[19] D. Peters, J. Chem. Phys. 1963, 38, 561-563.

[20] a) R. J. Abraham, T. A. D. Smith, W. A. Thomas, J. Chem. Soc. Perkin Trans. 2 1996, 1949-1955; b) F. Lahmani, A. Zehnacker, G. Denisov, G. G. Furin, J. Phys. Chem. 1996, 100, 8633-8639; c) T. A. Evans, K. R. Seddon, Chem. Commun. 1997, 2023-2024; d) M. Pham, M. Gdaniec, T. Polonski, J. Org. Chem. 1998, 63, 3731-3734; e) H. J. Schneider, Chem. Sci. 2012, 3, 1381-1394; f) J. D. Dunitz, ChemBioChem 2004, 5, 614-621.

[21] A. Berkessel, J. A. Adrio, D. Huettenhain, J. M. Neudorfl, J. Am. Chem. Soc. 2006, 128, 8421-8426.

[22] a) J. A. Olsen, D. W. Banner, P. Seiler, U. O. Sander, A. D'Arcy, M. Stihle, K. Muller, F. Diederich, Angew. Chem. Int. Ed. 2003, 42, 2507-2511; b) F. Hof, D. M. Scofield, W. B. Schweizer, F. Diederich, Angew. Chem. Int. Ed. 2004, 43, 5056-5059; c) J. Olsen, P. Seiler, B. Wagner, H. Fischer, T. Tschopp, U. ObstSander, D. W. Banner, M. Kansy, K. Muller, F. Diederich, Org. Biomol. Chem. 2004, 2, 1339-1352; d) J. A. Olsen, D. W. Banner, P. Seiler, B. Wagner, T. Tschopp, U. Obst-Sander, M. Kansy, K. Muller, F. Diederich, ChemBioChem 2004, 5, 666-675; e) R. Paulini, K. Muller, F. Diederich, Angew. Chem. Int. Ed. 2005, 44, 1788-1805.

[23] a) A. Hoffmann-Roeder, E. Schweizer, J. Egger, P. Seiler, U. Obst-Sander, B. Wagner, M. Kansy, D. W. Banner, F. Diederich, ChemMedChem 2006, 1, 1205-1215; b) J. C. Biffinger, H. W. Kim, S. G. DiMagno, ChemBioChem 2004, 5, 622-627; c) C. Y. Kim, P. P. Chandra, A. Jain, D. W. Christianson, J. Am. Chem. Soc. 2001, 123, 9620-9627.

[24] a) K. H. Piepenbrink, O. Y. Borbulevych, R. F. Sommese, J. Clemens, K. M. Armstrong, C. Desmond, P. Do, B. M. Baker, Biochem. J. 2009, 423, 353-361; b) T. R. Burke, B. Ye, X. J. Yan, S. M. Wang, Z. C. Jia, L. Chen, Z. Y. Zhang, D. Barford, Biochemistry 1996, 35, 15989-15996; c) A. DerHovanessian, J. B. Doyon, A. Jain, P. R. Rablen, A. M. Sapse, Org. Lett. 1999, 1, 1359-1362; d) J. B. Doyon, A. Jain, Org. Lett. 1999, 1, 183-185; e) C. Y. Kim, J. S. Chang, J. B. Doyon, T. T. Baird, C. A. Fierke, A. Jain, D. W. Christianson, J. Am. Chem. Soc. 2000, 122, 12125- 
12134; f) F. Abbate, A. Casini, A. Scozzafava, C. T. Supuran, J. Enzyme Inhib. Med. Chem. 2003, 18, 303-308; g) Y. L. Chou, D. D. Davey, K. A. Eagen, B. D. Griedel, R. Karanjawala, G. B. Phillips, K. L. Sacchi, K. J. Shaw, S. C. Wu, D. Lentz, A. M. Liang, L. Trinh, M. M. Morrissey, M. J. Kochanny, Bioorg. Med. Chem. Lett. 2003, 13, 507-511; h) H. Koga, A. Itoh, S. Murayama, S. Suzue, T. Irikura, J. Med. Chem. 1980, 23, 1358-1363; i) C. C. Kotoris, M. J. Chen, S. D. Taylor, Bioorg. Med. Chem. Lett. 1998 8, 3275-3280; j) J. M. Domagala, L. D. Hanna, C. L. Heifetz, M. P. Hutt, T. F. Mich, J. P. Sanchez, M. Solomon, J. Med. Chem. 1986, 29, 394-404; k) J. M. Domagala, C. L. Heifetz, T. F. Mich, J. B. Nichols, J. Med. Chem. 1986, 29, 445-448; 1) G. L. Grunewald, M. R. Seim, J. Lu, M. Makboul, K. R. Criscione, J. Med. Chem. 2006, 49, 2939-2952; m) B. D. Roth, D. F. Ortwine, M. L. Hoefle, C. D. Stratton, D. R. Sliskovic, M. W. Wilson, R. S. Newton, J. Med. Chem. 1990, 33, 21-31; n) E. A. Meyer, R. K. Castellano, F. Diederich, Angew. Chem. Int. Ed. 2003, 42, 1210 1250; o) E. Schweizer, A. Hoffmann-Roder, K. Scharer, J. A. Olsen, C. Fah, P. Seiler, U. Obst-Sander, B. Wagner, M. Kansy, F. Diederich, ChemMedChem 2006, 1, 611-621.

[25] S. G. DiMagno, H. Sun, Curr. Top. Med. Chem. 2006, 6, 14731482.

[26] a) S. Kaufman, Biochim. Biophys. Acta 1961, 51, 619-621; b) G. Guroff, J. W. Daly, D. M. Jerina, J. Renson, B. Witkop, Udenfrie.S, Science 1967, 157, 1524-1530.

[27] a) W. E. Barnette, Crit. Rev. Biochem. 1984, 15, 201-235; b) T. D. Penning, J. J. Talley, S. R. Bertenshaw, J. S. Carter, P. W. Collins, S. Docter, M. J. Graneto, L. F. Lee, J. W. Malecha, J. M. Miyashiro, R. S. Rogers, D. J. Rogier, S. S. Yu, G. D. Anderson, E. G. Burton, J. N. Cogburn, S. A. Gregory, C. M. Koboldt, W. E. Perkins, K. Seibert, A. W. Veenhuizen, Y. Y. Zhang, P. C. Isakson, J. Med. Chem. 1997, 40, 1347-1365; c) M. VanHeek, C. F. France, D. S. Compton, R. L. McLeod, N. P. Yumibe, K. B. Alton, E. J. Sybertz, H. R. Davis, J. Pharmacol. Exp. Ther. 1997, 283, 157-163; d) J. W. Clader, J. Med. Chem. 2004, 47, 1-9.

G. Villalba, R. U. Ayres, H. Schroder, Journal of Industrial Ecology 2007, 11, 85-101. a) D. H. R. Barton, A. K. Ganguly, R. H. Hesse, S. N. Loo, M. M. Pechet, Chem. Commun. 1968, 806-808; b) W. A. Sheppard, Tetrahedron Lett. 1969, 10, 83-84; c) D. H. R. Barton, N. D. Westcott, H. T. Toh, G. Tarzia, R. H. Hesse, M. M. Pechet, J. Chem. Soc., Chem. Commun. 1972, 122-123; d) J. Airey, D. H. R. Barton, A. K. Ganguly, R. H. Hesse, M. M. Pechet, An. Quim. 1974, 70, 871-875.

[30] E. Differding, H. Ofner, Synlett 1991, 187-189.

[31] a) W. E. Barnette, J. Am. Chem. Soc. 1984, 106, 452-454; b) E. Differding, R. W. Lang, Tetrahedron Lett. 1988, 29, 6087-6090; c) E. Differding, R. W. Lang, Helv. Chim. Acta 1989, 72, 12481252; d) F. A. Davis, W. Han, Tetrahedron Lett. 1991, 32, 16311634; e) G. Resnati, D. D. Desmarteau, J. Org. Chem. 1991, 56, 4925-4929; f) F. A. Davis, P. Zhou, C. K. Murphy, Tetrahedron Lett. 1993, 34, 3971-3974.

[32] a) T. Umemoto, K. Kawada, K. Tomita, Tetrahedron Lett. 1986 27, 4465-4468; b) T. Umemoto, K. Tomita, Tetrahedron Lett. 1986, 27, 3271-3274; c) K. Tomita, K. Kawada, T. Umemoto, J. Fluorine Chem. 1987, 35, 52-52; d) T. Umemoto, K. Tomita, $J$. Fluorine Chem. 1987, 35, 14-14; e) T. Umemoto, G. Tomizawa, Tetrahedron Lett. 1987, 28, 2705-2708; f) T. Umemoto, S. Fukami, G. Tomizawa, K. Harasawa, K. Kawada, K. Tomita, J. Am. Chem. Soc. 1990, 112, 8563-8575; g) T. Umemoto, K. Harasawa, G. Tomizawa, K. Kawada, K. Tomita, Bull. Chem. Soc Jpn. 1991, 64, 1081-1092; h) T. Umemoto, G. Tomizawa, J. Org. Chem. 1995, 60, 6563-6570; i) T. Umemoto, M. Nagayoshi, K. Adachi, G. Tomizawa, J. Org. Chem. 1998, 63, 3379-3385.

[33] a) P. T. Nyffeler, S. G. Duron, M. D. Burkart, S. P. Vincent, C. H. Wong, Angew. Chem. Int. Ed. 2005, 44, 192-212; b) G. S. Lal, G. P. Pez, R. G. Syvret, Chem. Rev. 1996, 96, 1737-1755.

[34] a) M. J. Adam, B. D. Pate, T. J. Ruth, J. M. Berry, L. D. Hall, J. Chem. Soc., Chem. Commun. 1981, 733-733; b) M. J. Adam, J. M. Berry, L. D. Hall, B. D. Pate, T. J. Ruth, Can. J. Chem. 1983, 61, $658-660$; c) M. J. Adam, T. J. Ruth, S. Jivan, B. D. Pate, J Fluorine Chem. 1984, 25, 329-337; d) M. R. Bryce, R. D. Chambers, S. T. Mullins, A. Parkin, J. Fluorine Chem. 1984, 26, 533-534; e) M. R. Bryce, R. D. Chambers, S. T. Mullins, A. Parkin, J. Chem. Soc., Chem. Commun. 1986, 1623-1624; f) H. H. Coenen, S. M. Moerlein, J. Fluorine Chem. 1987, 36, 63-75; g) H. F. Hodson, D. J. Madge, D. A. Widdowson, Synlett 1992, 831832 ; h) D. P. Matthews, S. C. Miller, E. T. Jarvi, J. S. Sabol, J. R.
McCarthy, Tetrahedron Lett. 1993, 34, 3057-3060; i) M. A. Tius, J. K. Kawakami, Synth. Commun. 1992, 22, 1461-1471.

[35] a) G. W. M. Visser, B. W. Vonhalteren, J. D. M. Herscheid, G. A. Brinkman, A. Hoekstra, J. Chem. Soc., Chem. Commun. 1984 655-656; b) G. W. M. Visser, C. N. M. Bakker, B. W. Vonhalteren, J. D. M. Herscheid, G. A. Brinkman, A. Hoekstra, J. Org. Chem. 1986, 51, 1886-1889; c) K. P. Butin, Y. M. Kiselev, T. V. Magdesieva, O. A. Reutov, J. Organomet. Chem. 1982, 235 , 127-133.

[36] a) G. V. De Meio, J. T. Pinhey, J. Chem. Soc., Chem. Commun. 1990, 1065-1066; b) G. V. De Meio, J. Morgan, J. T. Pinhey, Tetrahedron 1993, 49, 8129-8138.

[37] a) A. P. Lothian, C. A. Ramsden, Synlett 1993, 753-755; b) M. Tredwell, V. Gouverneur, Org. Biomol. Chem. 2006, 4, 26-32; c) P. Diraddo, M. Diksic, D. Jolly, J. Chem. Soc., Chem. Commun. 1984, 159-160; d) M. Speranza, C. Y. Shiue, A. P. Wolf, D. S. Wilbur, G. Angelini, J. Fluorine Chem. 1985, 30, 97-107; e) P. L. Coe, A. M. Stuart, D. J. Moody, J. Fluorine Chem. 1998, 92, 2732; f) A. M. Stuart, P. L. Coe, D. J. Moody, J. Fluorine Chem. 1998, $88,179-184$.

[38] a) L. J. Diorazio, D. A. Widdowson, J. M. Clough, Tetrahedron 1992, 48, 8073-8088; b) C. Cazorla, E. Metay, B. Andrioletti, M. Lemaire, Tetrahedron Lett. 2009, 50, 3936-3938; c) V. V. Grushin, M. M. Kantor, T. P. Tolstaya, T. M. Shcherbina, Bull. Acad. Sci. USSR, Div. Chem. Sci. 1984, 33, 2130-2135.

[39] a) J. DeYoung, H. Kawa, R. J. Lagow, J. Chem. Soc., Chem. Commun. 1992, 811-812; b) V. Snieckus, F. Beaulieu, K. Mohri, W. Han, C. K. Murphy, F. A. Davis, Tetrahedron Lett. 1994, 35, 3465-3468; c) D. W. Slocum, P. Shelton, K. M. Moran, Synthesis 2005, 3477-3498.

[40] a) P. Anbarasan, H. Neumann, M. Beller, Angew. Chem. Int. Ed. 2010, 49, 2219-2222; b) S. Yamada, A. Gavryushin, P. Knochel Angew. Chem. Int. Ed. 2010, 49, 2215-2218; c) P. Anbarasan, H. Neumann, M. Beller, Chem. Asian J. 2010, 5, 1775-1778.

[41] T. Tian, W.-H. Zhong, S. Meng, X.-B. Meng, Z.-J. Li, J. Org. Chem. 2012.

[42] a) L. V. Desai, K. J. Stowers, M. S. Sanford, J. Am. Chem. Soc. 2008, 130, 13285-13293; b) K. J. Stowers, M. S. Sanford, Org. Lett. 2009, 11, 4584-4587.

[43] a) D. C. Powers, D. Y. Xiao, M. A. L. Geibel, T. Ritter, J. Am. Chem. Soc. 2010, 132, 14530-14536; b) D. C. Powers, T. Ritter, Nature Chemistry 2009, 1, 302-309.

[44] T. Furuya, H. M. Kaiser, T. Ritter, Angew. Chem. Int. Ed. 2008, 47, 5993-5996.

[45] a) T. Furuya, T. Ritter, J. Am. Chem. Soc. 2008, 130, 10060 10061; b) T. Furuya, D. Benitez, E. Tkatchouk, A. E. Strom, P. Tang, W. A. Goddard, III, T. Ritter, J. Am. Chem. Soc. 2010, 132, 3793-3807.

[46] a) M. A. Tius, J. K. Kawakami, Synlett 1993, 1993, 207-208; b) M. A. Tius, J. K. Kawakami, Tetrahedron 1995, 51, 3997-4010.

[47] T. Furuya, A. E. Strom, T. Ritter, J. Am. Chem. Soc. 2009, 131, 1662-1663.

[48] T. Furuya, T. Ritter, Org. Lett. 2009, 11, 2860-2863.

[49] P. Tang, T. Ritter, Tetrahedron 2011, 67, 4449-4454.

[50] P. Tang, T. Furuya, T. Ritter, J. Am. Chem. Soc. 2010, 132, 12150-12154.

[51] a) A. D. Dilman, P. A. Belyakov, M. I. Struchkova, D. E. Arkhipov, A. A. Korlyukov, V. A. Tartakovsky, J. Org. Chem. 2010, 75, 5367-5370; b) T. Wu, G. Yin, G. Liu, J. Am. Chem. Soc 2009, 131, 16354-16355; c) S. Qiu, T. Xu, J. Zhou, Y. Guo, G. Liu, J. Am. Chem. Soc. 2010, 132, 2856-2857; d) T. Xu, X. Mu, H. Peng, G. Liu, Angew. Chem. Int. Ed. 2011, 50, 8176-8179. [52] a) H. Peng, G. Liu, Org. Lett. 2011, 13, 772-775; b) T. Xu, G. Liu, Org. Lett. 2012, 14, 5416-5419.

[53] N. Shibata, T. Ishimaru, S. Nakamura, T. Toru, J. Fluorine Chem. 2007, 128, 469-483.

[54] a) S. T. Purrington, N. V. Lazaridis, C. L. Bumgardner, Tetrahedron Lett. 1986, 27, 2715-2716; b) R. D. Chambers, J. Hutchinson, J. Fluorine Chem. 1998, 89, 229-232.

[55] a) W. J. Middleton, E. M. Bingham, J. Am. Chem. Soc. 1980, 102, 4845-4846; b) W. J. Middleton, E. M. Bingham, J. Am. Chem. Soc. 1980, 102, 4845-4846.

[56] a) H. Gershon, S. G. Schulman, A. D. Spevack, J. Med. Chem 1967, 10, 536-541; b) B. L. Shapiro, M. M. Chrysam, J. Org. Chem. 1973, 38, 880-893; c) C. E. Inman, R. E. Oesterling, E. A. Tyczkowski, J. Am. Chem. Soc. 1958, 80, 6533-6535. S. Stavber, M. Zupan, J. Chem. Soc., Chem. Commun. 1981, 795796. 
[58] B. Zajc, M. Zupan, J. Org. Chem. 1982, 47, 573-575.

[59] a) G. S. Lal, J. Org. Chem. 1993, 58, 2791-2796; b) F. A. Davis, W. Han, C. K. Murphy, J. Org. Chem. 1995, 60, 4730-4737.

[60] D. Cahard, C. Audouard, J. C. Plaquevent, N. Roques, Org. Lett 2000, 2, 3699-3701.

[61] a) L. Hintermann, A. Togni, Angew. Chem. Int. Ed. 2000, 39 , 4359-4362; b) L. Hintermann, M. Perseghini, A. Togni, Beil. J. Org. Chem. 2011, 7, 1421-1435; c) S. Piana, I. Devillers, A. Togni, U. Rothlisberger, Angew. Chem. Int. Ed. 2002, 41, 979982; d) R. Frantz, L. Hintermann, M. Perseghini, D. Broggini, A. Togni, Org. Lett. 2003, 5, 1709-1712; e) A. Bertogg, L. Hintermann, D. P. Huber, M. Perseghini, M. Sanna, A. Togni, Helv. Chim. Acta 2012, 95, 353-403.

[62] J. A. Ma, D. Cahard, Tetrahedron: Asymmetry 2004, 15, 1007 1011.

[63] N. Shibata, J. Kohno, K. Takai, T. Ishimaru, S. Nakamura, T. Toru, S. Kanemasa, Angew. Chem. Int. Ed. 2005, 44, 4204-4207.

[64] a) M. Althaus, C. Becker, A. Togni, A. Mezzetti, Organometallics 2007, 26, 5902-5911; b) M. Althaus, A. Togni, A. Mezzetti, J. Fluorine Chem. 2009, 130, 702-707.

[65] S. Suzuki, H. Furuno, Y. Yokoyama, J. Inanaga, Tetrahedron Asymmetry 2006, 17, 504-507.

[66] D. S. Reddy, N. Shibata, J. Nagai, S. Nakamura, T. Toru, S Kanemasa, Angew. Chem. Int. Ed. 2008, 47, 164-168.

[67] S. Suzuki, Y. Kitamura, S. Lectard, Y. Hamashima, M. Sodeoka, Angew. Chem. Int. Ed. 2012, 51, 4581-4585.

[68] a) Y. Hamashima, K. Yagi, H. Takano, L. Tamas, M. Sodeoka, J. Am. Chem. Soc. 2002, 124, 14530-14531; b) Y. Hamashima, H. Takano, D. Hotta, M. Sodeoka, Org. Lett. 2003, 5, 3225-3228.

[69] a) Y. Hamashima, T. Suzuki, Y. Shimura, T. Shimizu, N. Umebayashi, T. Tamura, N. Sasamoto, M. Sodeoka, Tetrahedron Lett. 2005, 46, 1447-1450; b) Y. Hamashima, T. Suzuki, H. Takano, Y. Shimura, Y. Tsuchiya, K.-i. Moriya, T. Goto, M. Sodeoka, Tetrahedron 2006, 62, 7168-7179.

[70] Y. Hamashima, T. Suzuki, H. Takano, Y. Shimura, M. Sodeoka, J. Am. Chem. Soc. 2005, 127, 10164-10165.

[71] T. Suzuki, T. Goto, Y. Hamashima, M. Sodeoka, J. Org. Chem. 2007, 72, 246-250.

[72] N. Shibata, E. Suzuki, Y. Takeuchi, J. Am. Chem. Soc. 2000, 122 10728-10729.

[73] N. Shibata, E. Suzuki, T. Asahi, M. Shiro, J. Am. Chem. Soc. 2001, 123, 7001-7009.

[74] N. Shibata, T. Ishimaru, E. Suzuki, K. L. Kirk, J. Org. Chem. 2003, 68, 2494-2497.

[75] J. Li, Y. Cai, W. Chen, X. Liu, L. Lin, X. Feng, J. Org. Chem. 2012, 77, 9148-9155.

[76] J. Xu, Y. Hu, D. Huang, K.-H. Wang, C. Xu, T. Niu, Adv. Synth Catal. 2012, 354, 515-526.

[77] D. Enders, M. R. M. Huttl, Synlett 2005, 991-993.

[78] T. D. Beeson, D. W. C. MacMillan, J. Am. Chem. Soc. 2005, 127, 8826-8828.

[79] M. Marigo, D. I. Fielenbach, A. Braunton, A. Kjoersgaard, K. A. Jorgensen, Angew. Chem. Int. Ed. 2005, 44, 3703-3706.

[80] D. D. Steiner, N. Mase, C. F. Barbas, Angew. Chem. Int. Ed. 2005 , 44, 3706-3710

[81] P. Kwiatkowski, T. D. Beeson, J. C. Conrad, D. W. C. MacMillan, J. Am. Chem. Soc. 2011, 133, 1738-1741.

[82] M. L. Schulte, C. W. Lindsley, Org. Lett. 2011, 13, 5684-5687.

[83] J. Erb, D. H. Paull, T. Dudding, L. Belding, T. Lectka, J. Am. Chem. Soc. 2011, 133, 7536-7546.

[84] a) A. W. Herriott, D. Picker, J. Am. Chem. Soc. 1975, 97, $2345-$ 2349; b) M. Makosza, Pure Appl. Chem. 2000, 72, 1399-1403; c) T. Hashimoto, K. Maruoka, Chem. Rev. 2007, 107, 5656-5682. D. Y. Kim, E. J. Park, Org. Lett. 2002, 4, 545-547. H. Jiang, A. Falcicchio, K. L. Jensen, M. W. Paixao, S. Bertelsen, K. A. Jorgensen, J. Am. Chem. Soc. 2009, 131, 7153-7157. M. Yamanaka, M. Arisawa, A. Nishida, M. Nakagawa, Tetrahedron Lett. 2002, 43, 2403 a) L. Carroll, M. C. Pacheco, L. Garcia, V. Gouverneur, Chem. Commun. 2006, 4113; b) G. Giuffredi, C. Bobbio, V. Gouverneur, J. Org. Chem. 2006, 71, 5361; c) Y. H. Lam, C. Bobbio, I. R. Cooper, V. Gouverneur, Angew. Chem. Int. Ed. 2007, 46, 5106; d) Y. h. Lam, M. N. Hopkinson, S. J. Stanway, V. Gouverneur, Synlett 2007, 3022; e) S. Purser, B. Odell, T. D. W. Claridge, P. R. Moore, V. Gouverneur, Chem. Eur. J. 2006, 12, 9176; f) S. Purser, C. Wilson, P. R. Moore, V. Gouverneur, Synlett 2007, 1166; g) H. Teare, F. Huguet, M. Tredwell, S. Thibaudeau, S. Luthra, V. Gouverneur, ARKIVOC 2007, 10, 232; h) H. Teare, E. G. Robins,
E. Årstad, S. K. Luthra, V. Gouverneur, Chem. Commun. 2007, 2330; i) M. Tredwell, V. Gouverneur, Org. Biomol. Chem. 2006 4, 26; j) M. Tredwell, K. Tenza, M. C. Pacheco, V. Gouverneur, Org. Lett. 2005, 7, 4495.

[89] S. Thibaudeau, V. Gouverneur, Org. Lett. 2003, 5, 4891-4893.

[90] B. Greedy, J. M. Paris, T. Vidal, V. Gouverneur, Angew. Chem. Int. Ed. 2003, 42, 3291-3294.

[91] a) N. P. Mankad, F. D. Toste, Chem. Sci. 2012, 3, 72-76; b) K. L. Hull, W. Q. Anani, M. S. Sanford, J. Am. Chem. Soc. 2006, 128, 7134-7135; c) J. M. Racowski, J. B. Gary, M. S. Sanford, Angew. Chem. Int. Ed. 2012, 51, 3414-3417.

[92] O. Lozano, G. Blessley, T. M. del Campo, A. L. Thompson, G. T. Giuffredi, M. Bettati, M. Walker, R. Borman, V. Gouverneur, Angew. Chem. Int. Ed. 2011, 50, 8105-8109.

[93] V. Rauniyar, A. D. Lackner, G. L. Hamilton, F. D. Toste, Science 2011, 334, 1681-1684.

[94] R. J. Phipps, K. Hiramatsu, F. D. Toste, J. Am. Chem. Soc. 2012 134, 8376-8379.

[95] T. Honjo, R. J. Phipps, V. Rauniyar, F. D. Toste, Angew. Chem. Int. Ed. 2012, 51, 9684-9688.

[96] a) W. Liu, X. Huang, M.-J. Cheng, R. J. Nielsen, W. A. Goddard, 3rd, J. T. Groves, Science 2012, 337, 1322-1325; b) K. B. McMurtrey, J. M. Racowski, M. S. Sanford, Org. Lett. 2012, 14 , 4094-4097.

[97] C. P. Andrieux, E. Differding, M. Robert, J. M. Saveant, J. Am. Chem. Soc. 1993, 115, 6592-6599.

[98] M. Rueda-Becerril, C. Chatalova Sazepin, J. C. T. Leung, T. Okbinoglu, P. Kennepohl, J.-F. Paquin, G. M. Sammis, J. Am Chem. Soc. 2012, 134, 4026-4029.

[99] T. B. Patrick, K. K. Johri, D. H. White, J. Org. Chem. 1983, 48, 4158-4159.

[100] F. Yin, Z. Wang, Z. Li, C. Li, J. Am. Chem. Soc. 2012, 1040110404

[101] J. C. T. Leung, C. Chatalova-Sazepin, J. G. West, M. RuedaBecerril, J.-F. Paquin, G. M. Sammis, Angew. Chem. Int. Ed. 2012, 51, 10804-10807.

[102] C. B. McPake, G. Sandford, Org. Process Res. Dev. 2012, 16 844-851.

[103] a) C. L. Liotta, H. P. Harris, J. Am. Chem. Soc. 1974, 96, 22502252; b) J. H. Clark, Chem. Rev. 1980, 80, 429-452.

[104] D. W. Kim, C. E. Song, D. Y. Chi, J. Am. Chem. Soc. 2002, 124, 10278-10279.

[105] a) D. W. Kim, D.-S. Ahn, Y.-H. Oh, S. Lee, H. S. Kil, S. J. Oh, S. J. Lee, J. S. Kim, J. S. Ryu, D. H. Moon, D. Y. Chi, J. Am. Chem. Soc. 2006, 128, 16394-16397; b) D. W. Kim, H.-J. Jeong, S. T. Litn, M.-H. Sohn, J. A. Katzenellenbogen, D. Y. Chi, J. Org Chem. 2008, 73, 957-962.

[106] H. R. Sun, S. G. DiMagno, J. Am. Chem. Soc. 2005, 127, 20502051.

[107] a) W. A. Sheppard, J. Am. Chem. Soc. 1960, 82, 4751-4752; b) W. A. Sheppard, J. Am. Chem. Soc. 1962, 84, 3058-3063; c) L. N. Markovskij, V. E. Pashinnik, A. V. Kirsanov, Synthesis 1973, 787-789; d) K. C. Mange, W. J. Middleton, J. Fluorine Chem. 1989, 43, 405-413; e) P. A. Messina, K. C. Mange, W. J. Middleton, J. Fluorine Chem. 1989, 42, 137-143; f) G. S. Lal, G. P. Pez, R. J. Pesaresi, F. M. Prozonic, Chem. Commun. 1999, 215-216; g) X. Bi, Synlett 2006, 2515-2516; h) T. Umemoto, R. P. Singh, Y. Xu, N. Saito, J. Am. Chem. Soc. 2010, 132, 1819918205.

[108] a) H. Hayashi, H. Sonoda, K. Fukumura, T. Nagata, Chem. Commun. 2002, 1618-1619; b) P. Tang, W. Wang, T. Ritter, J. Am. Chem. Soc. 2011, 133, 11482-11484.

[109] a) G. A. Olah, J. T. Welch, Y. D. Vankar, M. Nojima, I. Kerekes, J. A. Olah, J. Org. Chem. 1979, 44, 3872-3881; b) G. A. Olah, X. Y. Li, Q. Wang, G. K. S. Prakash, Synthesis 1993, 693-699; c) C. York, G. K. S. Prakash, G. A. Olah, Tetrahedron 1996, 52, 9-14; d) I. Bucsi, B. Torok, A. I. Marco, G. Rasul, G. K. S. Prakash, G. A. Olah, J. Am. Chem. Soc. 2002, 124, 7728-7736; e) G. Haufe, J. Prakt. Chem. 1996, 338, 99-113; f) T. Yoshiyama, T. Fuchigami, Chem. Lett. 1992, 1995-1998; g) T. Fuchigami, T. Fujita, J. Org. Chem. 1994, 59, 7190-7192.

[110] T. Sawamura, K. Takahashi, S. Inagi, T. Fuchigami, Angew. Chem. Int. Ed. 2012, 51, 4413-4416.

[111] J. Meisenheimer, Justus Liebigs Ann. Chem. 1902, 323, 205-246.

[112] B. Langlois, L. Gilbert, G. Forat in Industrial Chemistry Library, Vol. Volume 8 (Eds.: D. Jean-Roger, R. Serge), Elsevier, 1996, p. 244-292. 

1190.

[114] G. C. Finger, R. E. Oesterling, J. Am. Chem. Soc. 1956, 78, 2593 2596.

[115] H. B. Gottlieb, J. Am. Chem. Soc. 1936, 58, 532-533.

[116] G. C. Finger, C. W. Kruse, J. Am. Chem. Soc. 1956, 78, 6034 6037.

[117] H. Sun, S. G. DiMagno, Angew. Chem. Int. Ed. 2006, 45, 2720 2725.

[118] V. V. Grushin, W. J. Marshall, Organometallics 2008, 27, 48254828.

[119] a) E. C. Taylor, E. C. Bigham, D. K. Johnson, A. McKillop, J Org. Chem. 1977, 42, 362-363; b) I. F. Gun'kin, A. Y. Pankst'yanov, S. S. Popova, V. I. Kleimenova, Russ. J. Gen. Chem. 2000, 70, 37-39.

[120] M. Vanderpuy, J. Fluorine Chem. 1982, 21, 385-392.

[121] a) V. V. Grushin, Chem. Eur. J. 2002, 8, 1006-1014; b) V. V. Grushin, W. J. Marshall, Organometallics 2007, 26, 4997-5002; c) V. V. Grushin, Acc. Chem. Res. 2010, 43, 160-171; d) D. V. Yandulov, N. T. Tran, J. Am. Chem. Soc. 2007, 129, 1342-1358; e) S.-B. Zhao, R.-Y. Wang, H. Nguyen, J. J. Becker, M. R. Gagne, Chem. Commun. 2012, 48, 443-445; f) A. Casitas, M. Canta, M. Sola, M. Costas, X. Ribas, J. Am. Chem. Soc. 2011, 133, 1938619392; g) D. A. Watson, M. J. Su, G. Teverovskiy, Y. Zhang, J. Garcia-Fortanet, T. Kinzel, S. L. Buchwald, Science 2009, 325 , 1661-1664; h) T. Noel, T. J. Maimone, S. L. Buchwald, Angew. Chem. Int. Ed. 2011, 50, 8900-8903; i) P. Barthazy, L. Hintermann, R. M. Stoop, M. Worle, A. Mezzetti, A. Togni, Helv. Chim. Acta 1999, 82, 2448-2453; j) P. Barthazy, R. M. Stoop, M. Worle, A. Togni, A. Mezzetti, Organometallics 2000, 19, 28442852.

[122] T. J. Maimone, P. J. Milner, T. Kinzel, Y. Zhang, M. K. Takase, S. L. Buchwald, J. Am. Chem. Soc. 2011, 133, 18106-18109. J. Cardinale, J. Ermert, F. Kügler, A. Helfer, M. R. Brandt, H. H. Coenen, J. Labelled Compd. Radiopharm. 2012, 55, 450-453. P. S. Fier, J. F. Hartwig, J. Am. Chem. Soc. 2012, 10795-10798.

[124] P. Nemoto, T. Nishiyama, S. Akai, Org. Lett. 2011, 13, 2714 2717.

[126] a) N. Ishikawa, T. Kitazume, T. Yamazaki, Y. Mochida, T. Tatsuno, Chem. Lett. 1981, 761-764; b) D. P. Cox, J. Terpinski, W. Lawrynowicz, J. Org. Chem. 1984, 49, 3216-3219; c) M. Shimizu, Y. Nakahara, H. Yoshioka, Tetrahedron Lett. 1985, 26, 4207-4210; d) A. S. Pilcher, H. L. Ammon, P. Deshong, J. Am. Chem. Soc. 1995, 117, 5166-5167.

[127] C. F. Ye, J. M. Shreeve, J. Fluorine Chem. 2004, 125, 1869-1872.

[128] M. Kuroboshi, T. Hiyama, Tetrahedron Lett. 1991, 32, 12151218.

[129] a) T. Hamatani, S. Matsubara, H. Matsuda, M. Schlosser, Tetrahedron 1988, 44, 2875-2881; b) M. Nicoletti, D. O'Hagan, A. M. Z. Slawin, J. Am. Chem. Soc. 2005, 127, 482-483; c) L. Hunter, D. O'Hagan, A. M. Z. Slawin, J. Am. Chem. Soc. 2006, 128, 16422-16423; d) L. Hunter, D. O'Hagan, Org. Biomol. Chem. 2008, 6, 2843-2848.

[130] a) S. Noritake, N. Shibata, H. Kawai, M. K. Pandy, S. Nakamura, T. Toru, Heterocycl. Commun. 2009, 15, 105-113; b) B. Duthion, D. G. Pardo, J. Cossy, Org. Lett. 2010, 12, 4620-4623; c) G. Alvernhe, S. Lacombe, A. Laurent, Tetrahedron Lett. 1980, 21, 289-292.

[131] D. O'Hagan, J. Org. Chem. 2012, 77, 3689-3699.

[132] a) W. C. Smith, C. W. Tullock, E. L. Muetterties, W. R. Hasek, F. S. Fawcett, V. A. Engelhardt, D. D. Coffman, J. Am. Chem. Soc. 1959, 81, 3165-3166; b) W. R. Hasek, W. C. Smith, V. A. Engelhardt, J. Am. Chem. Soc. 1960, 82, 543-551.

[133] a) T. J. Tewson, M. J. Welch, J. Org. Chem. 1978, 43, 10901092; b) K. Boulton, B. E. Cross, J. Chem. Soc., Perkin Trans. 1 1979, 1354-1357; c) S. Castillon, A. Dessinges, R. Faghih, G. Lukacs, A. Olesker, T. T. Thang, J. Org. Chem. 1985, 50, 49134917; d) G. H. Posner, S. R. Haines, Tetrahedron Lett. 1985, 26, 5-8; e) S. V. Pansare, J. C. Vederas, J. Org. Chem. 1987, 52, 4804-4810; f) D. F. Shellhamer, D. T. Anstine, K. M. Gallego, B. R. Ganesh, A. A. Hanson, K. A. Hanson, R. D. Henderson, J. M. Prince, V. L. Heasley, J. Chem. Soc., Perkin Trans. 2 1995, 861866; g) G. Hagele, A. Haas, J. Fluorine Chem. 1996, 76, 15-19; h) D. F. Shellhamer, A. A. Briggs, B. M. Miller, J. M. Prince, D. H. Scott, V. L. Heasley, J. Chem. Soc., Perkin Trans. 2 1996, 973-977; i) S. B. Ferreira, Synlett 2006, 1130-1131; j) M. Hudlicky, J. Fluorine Chem. 1987, 36, 373-384; k) A. J. Ratcliffe, I. Warner, Tetrahedron Lett. 1995, 36, 3881-3884; 1) S. Das, S.
Chandrasekhar, J. S. Yadav, R. Gree, Tetrahedron Lett. 2007, 48, 5305-5307; m) W. J. Middleton, E. M. Bingham, J. Org. Chem. 1980, 45, 2883-2887; n) M. Kirihara, K. Niimi, T. Momose, Chem. Commun. 1997, 599-600; o) M. Kirihara, K. Niimi, M. Okumura, T. Momose, Chemical \& Pharmaceutical Bulletin 2000, 48, 220-222.

[134] A. Sutherland, J. C. Vederas, Chem. Commun. 1999, 1739-1740.

[135] B. Bennua-Skalmowski, H. Vorbrüggen, Tetrahedron Lett. 1995, 36, 2611-2614.

[136] J. J. Yin, D. S. Zarkowsky, D. W. Thomas, M. M. Zhao, M. A. Huffman, Org. Lett. 2004, 6, 1465-1468.

[137] N. N. Yarovenko, M. A. Raksha, Zh. Obshch. Khim. 1959, 29 2159-2163.

[138] A. Takaoka, H. Iwakiri, N. Ishikawa, Bull. Chem. Soc. Jpn. 1979 52, 3377-3380

[139] V. A. Petrov, S. Swearingen, W. Hong, W. Chris Petersen, J. Fluorine Chem. 2001, 109, 25-31.

[140] R. Surmont, G. Verniest, A. De Groot, J. W. Thuring, N. De Kimpe, Adv. Synth. Catal. 2010,352, 2751-2756.

[141] a) G. S. Lal, G. P. Pez, R. J. Pesaresi, F. M. Prozonic, H. S. Cheng, J. Org. Chem. 1999, 64, 7048-7054; b) R. P. Singh, J. M. Shreeve, Org. Lett. 2001, 3, 2713-2715; c) R. P. Singh, J. M. Shreeve, Synthesis 2002, 2561-2578; d) R. P. Singh, J. M. Shreeve, J. Fluorine Chem. 2002, 116, 23-26; e) R. P. Singh, B. Twamley, J. M. Shreeve, J. Org. Chem. 2002, 67, 1918-1924; f) R. P. Singh, J. M. Shreeve, J. Org. Chem. 2003, 68, 6063-6065; g) G. R. Krow, G. L. Lin, K. P. Moore, A. M. Thomas, C. DeBrosse, C. W. Ross, H. G. Ramjit, Org. Lett. 2004, 6, 16691672; h) Y. Chang, A. Tewari, A.-I. Adi, C. Bae, Tetrahedron 2008, 64, 9837-9842; i) G. S. Lal, E. Lobach, A. Evans, J. Org. Chem. 2000, 65, 4830-4832.

[142] a) R. Sasson, A. Hagooly, S. Rozen, Org. Lett. 2003, 5, 769-771; b) S. Rozen, Acc. Chem. Res. 2005, 38, 803-812; c) Y. Hagooly, S. Rozen, J. Org. Chem. 2008, 73, 6780-6783; d) Y. Hagooly, R. Sasson, M. J. Welch, S. Rozen, Eur. J. Org. Chem. 2008, 28752880; e) O. Cohen, Y. Hagooly, S. Rozen, Tetrahedron 2009, 65, 1361-1365; f) U. Farooq, A.-u.-H. A. Shah, T. Wirth, Angew. Chem. Int. Ed. 2009, 48, 1018-1020; g) Y. Hagooly, O. Cohen, S. Rozen, Tetrahedron Lett. 2009, 50, 392-394.

[143] R. P. Singh, T. Umemoto, J. Org. Chem. 2011, 76, 3113-3121.

[144] F. Beaulieu, L.-P. Beauregard, G. Courchesne, M. Couturier, F. LaFlamme, A. L'Heureux, Org. Lett. 2009, 11, 5050-5053.

[145] A. L'Heureux, F. Beaulieu, C. Bennett, D. R. Bill, S. Clayton, F. LaFlamme, M. Mirmehrabi, S. Tadayon, D. Tovell, M. Couturier, J. Org. Chem. 2010, 75, 3401-3411.

[146] W. J. Middleton, J. Org. Chem. 1975, 40, 574-578.

[147] a) F. Benayoud, D. J. deMendonca, C. A. Digits, G. A. Moniz, T. C. Sanders, G. B. Hammond, J. Org. Chem. 1996, 61, 5159; b) A M. Kornilov, A. E. Sorochinskii, V. P. Kukhar, Zh. Org. Khim 1989, 25, 2520-2523; c) T. Asai, Y. Morizawa, T. Shimada, T. Nakayama, M. Urushihara, Y. Matsumura, A. Yasuda, Tetrahedron Lett. 1995, 36, 273; d) Y. Matsumura, T. Asai, T. Shimada, T. Nakayama, M. Urushihara, Y. Morizawa, A. Yasuda, T. Yamamoto, B. Fujitani, K. Hosoki, Chem. Pharm. Bull. 1995, 43, 353; e) Y. Matsumura, T. Nakano, T. Asai, Y. Morizawa, ACS Symp. Ser. 1996, 639, 83; f) Y. Matsumura, T. Shimada, T Nakayama, M. Urushihara, T. Asai, Y. Morizawa, A. Yasuda, Tetrahedron 1995, 51, 8771; g) Y. Matsumura, T. Shimada, S. Z. Wang, T. Asai, Y. Morizawa, A. Yasuda, Bull. Chem. Soc. Jpn. 1996, 69, 3523; h) E. Haloui, C. B. Nasr, D. Canet, J. Mol. Struct. 1982, 95, 231-235; i) A. Khanous, A. Gorgues, J. Cousseau, J. Fluorine Chem. 1990, 49, 401-408; j) M. Shimizu, S. Kanemoto, Y. Nakahara, Heterocycles 2000, 52, 117.

[148] R. E. A. Dear, E. E. Gilbert, J. Org. Chem. 1968, 33, 819-823.

[149] L. I. Zakharkin, B. A. Kvasov, V. N. Lebedev, Zh. Obshch. Khim. 1971, 41, 2694 .

[150] L. F. Tietze, U. Bothe, I. Schuberth, Chem. Eur. J. 2000, 6, 836.

[151] a) M. Prakesch, D. Grée, R. Grée, J. Org. Chem. 2001, 66, 3146; b) M. Prakesch, E. Kerouredan, D. Grée, R. Grée, J. DeChancie, K. N. Houk, J. Fluorine Chem. 2004, 125, 537; c) M. Prakesch, D. Grée, R. Grée, Acc. Chem. Res. 2002, 35, 175; d) D. Grée, V. Madiot, R. Grée, Tetrahedron Lett. 1999, 40, 6399; e) V. Madiot, D. Grée, R. Grée, Tetrahedron Lett. 1999, 40, 6403; f) V. Madiot, P. Lesot, D. Grée, J. Courtieu, R. Grée, Chem. Commun. 2000, 169; g) V. L. Manthati, A. S. K. Murthy, F. Caijo, D. Drouin, P. Lesot, D. Grée, R. Grée, Tetrahedron: Asymmetry 2006, 17, 2306. 
[152] a) Z. Wang, Y. Gu, A. J. Zapata, G. B. Hammond, J. Fluorine Chem. 2001, 107, 127; b) A. J. Zapata, Y. Gu, G. B. Hammond, J. Org. Chem. 2000, 65, 227.

[153] a) A. Boukerb, D. Grée, M. Laabassi, R. Grée, J. Fluorine Chem. 1998, 88, 23; b) D. Grée, L. Vallerie, R. Grée, L. Toupet, I. Washington, J. P. Pelicier, M. Villacampa, J. M. Pérez, K. N. Houk, J. Org. Chem. 2001, 66, 2374; c) G. M. Blackburn, D. E. Kent, J. Chem. Soc., Chem. Commun. 1981, 511-513; d) G. M. Blackburn, D. E. Kent, J. Chem. Soc., Perkin Trans. 1 1986, $913-$ 917; e) G. B. Hammond, D. J. deMendonca, J. Fluorine Chem. 2000, 102, 189.

[154] S. Legoupy, C. Crévisy, J. C. Guillemin, R. Grée, J. Fluorine Chem. 1999, 93, 171-173.

[155] D. M. Grée, C. J. M. Kermarrec, J. T. Martelli, R. L. Grée, J. P. Lellouche, L. J. Toupet, J. Org. Chem. 1996, 61, 1918.

[156] a) C. D. Poulter, P. L. Wiggins, T. L. Plummer, J. Org. Chem. 1981, 46, 1532-1538; b) J. Mann, G. P. Smith, J. Chem. Soc., Perkin Trans. 1 1991, 2884-2885.

[157] a) B. E. Cross, P. Hendley, J. Chem. Soc., Perkin Trans. 1 1975, 2523-2525; b) R. E. Banks, B. E. Cross, J. Chem. Soc., Perkin Trans. 1 1977, 5, 512-515; c) R. E. Banks, J. H. Bateson, B. E. Cross, A. Erasmusson, J. Chem. Res., Synop. 1980, 2, 46; d) A. P. Kozikowski, J. P. Wu, Tetrahedron Lett. 1990, 31, 4309-4312.

[158] F. Munyemana, A. M. Frisque-Hesbain, A. Devos, L. Ghosez,

[158] F. Munyemana, A. M. Frisque-Hesbain, A. Devos, L. Ghosez,
Tetrahedron Lett. 1989, 30, 3077-3080. N. Yoneda, T. Fukuhara, Chem. Lett. 2001, 222.

[160] a) D. P. Cox, J. Terpinski, W. Lawrynowicz, J. Org. Chem. 1984, 49, 3216-3219; b) P. S. Bhadury, M. Pandey, D. K. Jaiswal, J. Fluorine Chem. 1995, 73, 185; c) D. Guijarro, M. Yus, J. Organomet. Chem. 2001, 624, 53; d) D. Albanese, D. Landini, M. Penso, J. Org. Chem. 1998, 63, 9587; e) J. Villieras, M. Rambaud, Synthesis 1982, 924-926; f) J. Ichihara, T. Matsuo, T. Hanafusa, T. Ando, J. Chem. Soc., Chem. Commun. 1986, 793-794; g) J. Ichihara, Y. Takai, T. Hanafusa, K. Tomioka, J. Fluorine Chem. 1995, 71, 131; h) K. Bannai, T. Toru, T. Ōba, T. Tanaka, N. Okamura, K. Watanabe, A. Hazato, S. Kurozumi, Tetrahedron 1986, 42, 6735-6746; i) G. A. Olah, J. T. Welch, Y. D. Vankar, M. Nojima, I. Kerekes, J. A. Olah, J. Org. Chem. 1979, 44, 38723881; j) J. Mann, B. Pietrzak, J. Chem. Soc., Perkin Trans. 1 1983, 2681-2685; k) J. Ichikawa, K. i. Sugimoto, T. Sonoda, H. Kobayashi, Chem. Lett. 1987, 1985-1988.

[161] A. Hazari, V. Gouverneur, J. M. Brown, Angew. Chem. Int. Ed. 2009, 48, 1296-1299.

[162] L. Hintermann, F. Lang, P. Maire, A. Togni, Eur. J. Inorg. Chem. 2006, 1397-1412.

[163] M. H. Katcher, A. G. Doyle, J. Am. Chem. Soc. 2010, 132, 17402-17404.

[164] M. H. Katcher, A. Sha, A. G. Doyle, J. Am. Chem. Soc. 2011, 133, 15902-15905.

[165] J. Zhu, G. C. Tsui, M. Lautens, Angew. Chem. Int. Ed. 2012, 51, 12353-12356.

[166] J. J. Topczewski, T. J. Tewson, H. M. Nguyen, J. Am. Chem. Soc. 2011, 133, 19318-19321.

[167] C. Hollingworth, A. Hazari, M. N. Hopkinson, M. Tredwell, E. Benedetto, M. Huiban, A. D. Gee, J. M. Brown, V. Gouverneur, Angew. Chem. Int. Ed. 2011, 50, 2613-2617.

[168] A. M. Lauer, J. Wu, Org. Lett. 2012, 14, 5138-5141.

[169] G. Haufe, G. Alvernhe, A. Laurent, T. Ernet, O. Goj, S. Kroger, A. Sattler, Org. Synth. 1999, 76, 159-168.

[170] a) S. Bruns, G. Haufe, J. Fluorine Chem. 2000, 104, 247-254; b) G. Haufe, S. Bruns, Adv. Synth. Catal. 2002, 344, 165-171.

[171] a) J. A. Kalow, A. G. Doyle, J. Am. Chem. Soc. 2010, 132, 32683269; b) J. A. Kalow, A. G. Doyle, J. Am. Chem. Soc. 2011, 133, 16001-16012.

[172] a) G. M. Blackburn, T. D. Perree, A. Rashid, C. Bisbal, B. Lebleu, Chem. Scri. 1986, 26, 21-24; b) D. P. Phillion, D. G. Cleary, J. Org. Chem. 1992, 57, 2763-2764; c) D. B. Berkowitz, Q. R. Shen, J. H. Maeng, Tetrahedron Lett. 1994, 35, 6445-6448; d) D. B. Berkowitz, M. Bose, J. Fluorine Chem. 2001, 112, 13-33; e) J. Lapierre, V. Ahmed, M. J. Chen, M. Ispahany, J. G. Guillemette, S. D. Taylor, Bioorg. Med. Chem. Lett. 2004, 14, 151-155; f) T. R. Burke, Jr., Curr. Top. Med. Chem. 2006, 6, 1465-1471.

[173] G. K. S. Prakash, I. Ledneczki, S. Chacko, S. Ravi, G. A. Olah, J. Fluorine Chem. 2008, 129, 1036-1040.

[174] W. Zhang, L. Zhu, J. Hu, Tetrahedron 2007, 63, 10569-10575.

[175] G. K. S. Prakash, I. Ledneuki, S. Chacko, G. A. Olah, Org. Lett. 2008, $10,557-560$.
[176] Y. Nomura, E. Tokunaga, N. Shibata, Angew. Chem. Int. Ed. 2011, 50, 1885-1889.

[177] S. Stavber, M. Zupan, Tetrahedron Lett. 1993, 34, 4355-4356.

[178] M. Ochiai, A. Yoshimura, T. Mori, Y. Nishi, M. Hirobe, J. Am. Chem. Soc. 2008, 130, 3742-3743.

[179] Q. B. Lu, T. Benneche, Acta Chem. Scand. 1996, 50, 850-852.

[180] T. B. Patrick, K. K. Johri, D. H. White, W. S. Bertrand, R. Mokhtar, M. R. Kilbourn, M. J. Welch, Can. J. Chem. 1986, 64, 138-141.

[181] T. Umemoto, G. Tomizawa, Bull. Chem. Soc. Jpn. 1986, 59, 3625-3629.

[182] a) R. Ringom, T. Benneche, Acta Chem. Scand. 1999, 53, 41; b) C. T. Mason, C. C. Allain, J. Am. Chem. Soc. 1956, 78, 16821684; c) M. Schlosser, O. Desponds, R. Lehmann, E. Moret, G. Rauchschwalbe, Tetrahedron 1993, 49, 10175.

[183] K. M. More, J. Wemple, Synthesis 1977, 791-792.

[184] a) S. F. Wnuk, M. J. Robins, J. Org. Chem. 1990, 55, 4757-4760; b) M. J. Robins, S. F. Wnuk, J. Org. Chem. 1993, 58, 3800-3801. S. Furuta, M. Kuroboshi, T. Hiyama, Tetrahedron Lett. 1995, 36, 8243-8246.

[186] Y. Li, C. F. Ni, J. Liu, L. J. Zhang, J. Zheng, L. G. Zhu, J. B. Hu, Org. Lett. 2006, 8, 1693-1696.

[187] J. Liu, L. Zhang, J. Hu, Org. Lett. 2008, 10, 5377-5380.

[188] J. Liu, Y. Li, J. Hu, J. Org. Chem. 2007, 72, 3119-3121.

[189] a) X. Shen, L. Zhang, Y. Zhao, L. Zhu, G. Li, J. Hu, Angew. Chem. Int. Ed. 2011, 50, 2588-2592; b) T. Furukawa, Y. Goto, J. Kawazoe, E. Tokunaga, S. Nakamura, Y. Yang, H. Du, A. Kakehi, M. Shiro, N. Shibata, Angew. Chem. Int. Ed. 2010, 49, 1642-1647.

[190] J. Liu, C. Ni, Y. Li, L. Zhang, G. Wang, J. Hu, Tetrahedron Lett. 2006, 47, 6753-6756.

[191] G. K. S. Prakash, X. Zhao, S. Chacko, F. Wang, H. Vaghoo, G. A. Olah, Beil. J. Org. Chem. 2008, 4.

[192] Y. Arroyo, M. Ascension Sanz-Tejedor, A. Parra, J. L. Garcia Ruano, Chem. Eur. J. 2012, 18, 5314-5318.

[193] G. K. S. Prakash, S. Chacko, S. Alconcel, T. Stewart, T. Mathew, G. A. Olah, Angew. Chem. Int. Ed. 2007, 46, 4933-4936.

[194] G. K. S. Prakash, S. Chacko, H. Vaghoo, N. Shao, L. Gurung, T. Mathew, G. A. Olah, Org. Lett. 2009, 11, 1127-1130.

[195] C. Ni, J. Hu, Tetrahedron Lett. 2009, 50, 7252-7255.

[196] T. Fukuzumi, N. Shibata, M. Sugiura, H. Yasui, S. Nakamura, T. Toru, Angew. Chem. Int. Ed. 2006, 45, 4973-4977.

[197] Y. Zhao, B. Gao, C. Ni, J. Hu, Org. Lett. 2012, 14, 6080-6083.

[198] S. Mizuta, N. Shibata, Y. Goto, T. Furukawa, S. Nakamura, T. Toru, J. Am. Chem. Soc. 2007, 129, 6394-6395.

[199] G. K. S. Prakash, F. Wang, T. Stewart, T. Mathew, G. A. Olah, Proc. Natl. Acad. Sci. USA 2009, 106, 4090-4094.

[200] H. W. Moon, M. J. Cho, D. Y. Kim, Tetrahedron Lett. 2009, 50, 4896-4898.

[201] a) F. R. Leroux, B. Manteau, J.-P. Vors, S. Pazenok, Beil. J. Org. Chem. 2008, 4; b) B. Manteau, S. Pazenok, J.-P. Vors, F. R. Leroux, J. Fluorine Chem. 2010, 131, 140-158.

[202] J. A. Erickson, J. I. McLoughlin, J. Org. Chem. 1995, 60, 16261631.

[203] P. Cao, J. X. Duan, Q. Y. Chen, J. Chem. Soc., Chem. Commun. 1994, 737-738.

[204] J. Gonzalez, C. J. Foti, S. Elsheimer, J. Org. Chem. 1991, 56, $4322-4325$.

[205] I. Rico, D. Cantacuzene, C. Wakselman, Tetrahedron Lett. 1981, 22, 3405-3408.

[206] V. Reutrakul, T. Thongpaisanwong, P. Tuchinda, C. Kuhakarn, M. Pohmakotr, J. Org. Chem. 2004, 69, 6913-6915.

[207] a) Y. Li, J. Liu, L. Zhang, L. Zhu, J. Hu, J. Org. Chem. 2007, 72, 5824-5827; b) Y. Li, H. Li, J. Hu, Tetrahedron 2009, 65, 478-483.

[208] J. Zhu, F. Wang, J. Hu, Science China-Chemistry 2011, 54, 95102.

[209] X. Yang, X. Fang, X. Yang, M. Zhao, Y. Han, Y. Shen, F. Wu, Tetrahedron 2008, 64, 2259-2269.

[210] a) R. Miethchen, M. Hein, H. Reinke, Eur. J. Org. Chem. 1998 , 919-923; b) S. Tews, R. Miethchen, H. Reinke, Synthesis 2003, 707-716.

[211] K. Iseki, D. Asada, M. Takahashi, T. Nagai, Y. Kobayashi, Chemical \& Pharmaceutical Bulletin 1996, 44, 1314-1317.

[212] D. A. Nagib, M. E. Scott, D. W. C. MacMillan, J. Am. Chem. Soc. 2009, 131, 10875-10877.

[213] a) Y. Fujiwara, J. A. Dixon, R. A. Rodriguez, R. D. Baxter, D. D. Dixon, M. R. Collins, D. G. Blackmond, P. S. Baran, J. Am. Chem. Soc. 2012, 134, 1494-1497; b) Y. Fujiwara, J. A. Dixon, F. O'Hara, E. D. Funder, D. D. Dixon, R. A. Rodriguez, R. D. 
Baxter, B. Herle, N. Sach, M. R. Collins, Y. Ishihara, P. S. Baran, Nature 2012, 492, 95-99.

[214] a) R. F. Clark, J. H. Simons, J. Am. Chem. Soc. 1955, 77, 66186618; b) T. G. Miller, J. W. Thanassi, J. Org. Chem. 1960, 25, 2009-2012; c) I. Rico, C. Wakselman, Tetrahedron 1981, 37, 4209-4213; d) I. Rico, C. Wakselman, Tetrahedron Lett. 1981, 22 , 323-326; e) B. Langlois, J. Fluorine Chem. 1988, 41, 247-261; f) Q. Y. Chen, S. W. Wu, J. Fluorine Chem. 1989, 44, 433-440; g) Y. Zafrani, G. Sod-Moriah, Y. Segall, Tetrahedron 2009, 65, 5278-5283.

[215] J. Mizukado, Y. Matsukawa, H. D. Quan, M. Tamura, A. Sekiya, J. Fluorine Chem. 2006, 127, 400-404.

[216] K. Morimoto, K. Makino, G. Sakata, J. Fluorine Chem. 1992, 59, 417-422.

[217] E. Nawrot, A. Jonczyk, J. Fluorine Chem. 2006, 127, 943-947.

[218] G. K. S. Prakash, Z. Zhang, F. Wang, C. Ni, G. A. Olah, J. Fluorine Chem. 2011, 132, 792-798.

[219] J. W. Lyga, R. M. Patera, J. Fluorine Chem. 1998, 92, 141-145.

[220] K. I. Petko, L. M. Yagupolskii, J. Fluorine Chem. 2001, 108, 211-214

[221] K. I. Petko, A. A. Tolmachev, L. M. Yagupol'skii, Russ. J. Org. Chem. 2002, 38, 1030-1034.

[222] K. I. Petko, L. M. Yagupol'skii, Russ. J. Org. Chem. 2004, 40, 601-602.

[223] A. Jonczyk, E. Nawrot, M. Kisielewski, J. Fluorine Chem. 2005, 126, 1587-1591.

[224] M. Ando, T. Wada, N. Sato, Org. Lett. 2006, 8, 3805-3808.

[225] M. Pelc, W. Huang, J. Trujillo, J. Baldus, S. Turner, P. Kleine, S. Yang, A. Thorarensen, Synlett 2010, 219-222.

[226] a) D. J. Burton, D. M. Wiemers, J. Am. Chem. Soc. 1985, 107, 5014-5015; b) J. M. Paratian, E. Labbe, S. Sibille, J. Y. Nedelec, J. Perichon, J. Organomet. Chem. 1995, 487, 61-64; c) W. Tyrra, D. Naumann, J. Prakt. Chem. 1996, 338, 283-286; d) S. V. Pasenok, N. V. Kirij, Y. L. Yagupolskii, D. Naumann, W. Tyrra, A. Fitzner, Z. Anorg. Allg. Chem. 1999, 625, 834-838.

[227] M. A. Guerra, T. R. Bierschenk, R. J. Lagow, J. Am. Chem. Soc. 1986, 108, 4103-4105.

[228] L. Zhang, J. Zheng, J. Hu, J. Org. Chem. 2006, 71, 9845-9848.

[229] J. Zheng, Y. Li, L. Zhang, J. Hu, G. J. Meuzelaar, H.-J. Federsel, Chem. Commun. 2007, 5149-5151.

[230] a) G. K. S. Prakash, C. Weber, S. Chacko, G. A. Olah, Org. Lett. 2007, 9, 1863-1866; b) G. K. S. Prakash, C. Weber, S. Chacko, G. A. Olah, J. Comb. Chem. 2007, 9, 920-923.

[231] E. Nawrot, A. Jonczyk, J. Fluorine Chem. 2009, 130, 466-469.

[232] T. Y. Shen, S. Lucas, L. H. Sarett, Tetrahedron Lett. 1961, 43-47.

[233] K. Iseki, D. Asada, M. Takahashi, T. Nagai, Y. Kobayashi, Tetrahedron: Asymmetry 1996, 7, 1205-1215.

[234] a) M. Rapp, X. Cai, W. Xu, W. R. Dolbier, Jr., S. F. Wnuk, J. Fluorine Chem. 2009, 130, 321-328; b) W. R. Dolbier, F. Tian, J. X. Duan, A. R. Lia, S. Ait-Mohand, O. Bautista, S. Buathong, J. M. Baker, J. Crawford, P. Anselme, X. H. Cai, A. Modzelewska, H. Koroniak, M. A. Battiste, Q. Y. Chen, J. Fluorine Chem. 2004, 125, 459-469; c) F. Tian, V. Kruger, O. Bautista, J. X. Duan, A. R. Li, W. R. Dolbier, Q. Y. Chen, Org. Lett. 2000, 2, 563-564; d) F. Wang, W. Zhang, J. Zhu, H. Li, K.-W. Huang, J. Hu, Chem. Commun. 2011, 47, 2411-2413.

[235] X. H. Cai, Y. Zhai, I. Ghiviriga, K. A. Abboud, W. R. Dolbier, J. Org. Chem. 2004, 69, 4210-4215.

[236] a) W. Xu, K. A. Abboud, I. Ghiviriga, W. R. Dolbier, Jr., M. Rapp, S. F. Wnuk, Org. Lett. 2006, 8, 5549-5551; b) K. Fuchibe, Y. Koseki, T. Aono, H. Sasagawa, J. Ichikawa, J. Fluorine Chem. 2012, 133, 52-60.

[237] W. Zhang, F. Wang, J. Hu, Org. Lett. 2009, 11, 2109-2112.

[238] M. Ochiai, A. Yoshimura, M. M. Hoque, T. Okubo, M. Saito, K. Miyamoto, Org. Lett. 2011, 13, 5568-5571.

[239] K. Mikami, Y. Tomita, Y. Itoh, Angew. Chem. Int. Ed. 2010, 49, 3819-3822.

[240] D. Morris, Acta Crystallogr. 1956, 9, 197-198.

[241] Z. He, T. Luo, M. Hu, Y. Cao, J. Hu, Angew. Chem. Int. Ed. 2012, 51, 3944-3947.

[242] Q. Qi, Q. Shen, L. Lu, J. Am. Chem. Soc. 2012, 134, 6548-6551.

[243] a) M. Kuroboshi, T. Hiyama, Synlett 1994, 251; b) A. Haas, M. Spitzer, M. Lieb, Chem. Ber. 1988, 121, 1329-1340.

[244] D. C. England, L. R. Melby, M. A. Dietrich, R. V. Lindsey, J. Am. Chem. Soc. 1960, 82, 5116-5122.

[245] P. Kirsch, M. Bremer, A. Taugerbeck, T. Wallmichrath, Angew. Chem. Int. Ed. 2001, 40, 1480-1484.

[246] P. E. Aldrich, W. A. Sheppard, J. Org. Chem. 1964, 29, 11-15. a) G. K. S. Prakash, J. B. Hu, W. Ying, G. A. Olah, Org. Lett. 2004, 6, 4315-4317; b) G. K. S. Prakash, J. B. Hu, Y. Wang, G. A. Olah, Eur. J. Org. Chem. 2005, 2218-2223; c) G. K. S. Prakash, C. Ni, F. Wang, J. Hu, G. A. Olah, Angew. Chem. Int. Ed. 2011, $50,2559-2563$.

[248] Y. Zhao, B. Gao, J. Hu, J. Am. Chem. Soc. 2012, 134, 5790-5793. [249] Y. Li, J. Hu, Angew. Chem. Int. Ed. 2007, 46, 2489-2492.

[250] C. Ni, J. Liu, L. Zhang, J. Hu, Angew. Chem. Int. Ed. 2007, 46, 786-789.

[251] L. Zhu, Y. Li, C. Ni, J. Hu, P. Beier, Y. Wang, G. K. S. Prakash, G. A. Olah, J. Fluorine Chem. 2007, 128, 1241-1247.

[252] P. Beier, A. V. Alexandrova, M. Zibinsky, G. K. S. Prakash, Tetrahedron 2008, 64, 10977-10985.

[253] a) J. Hine, J. J. Porter, J. Am. Chem. Soc. 1960, 82, 6178-6181; b) M. Zupan, J. Fluorine Chem. 1976, 8, 305-309.

[254] a) G. K. S. Prakash, J. Hu, Acc. Chem. Res. 2007, 40, 921-930; b) J. Hu, J. Fluorine Chem. 2009, 130, 1130-1139.

[255] C. Ni, F. Wang, J. Hu, Beil. J. Org. Chem. 2008, 4.

[256] N. Wiberg, Angew. Chem. Int. Ed. 1968, 7, 766-779.

[257] G. K. S. Prakash, Y. Wang, J. B. Hu, G. A. Olah, J. Fluorine Chem. 2005, 126, 1361-1367.

[258] a) T. Hagiwara, T. Fuchikami, Synlett 1995, 717-718; b) G. Bissky, V. I. Staninets, A. A. Kolomeitsev, G. V. Roschenthaler, Synlett 2001, 374-378; c) C. F. Ni, J. B. Hu, Tetrahedron Lett. 2005, 46, 8273-8277; d) G. K. S. Prakash, J. B. Hu, Y. Wang, G. A. Olah, J. Fluorine Chem. 2005, 126, 527-532; e) S. Mizuta, N. Shibata, S. Ogawa, H. Fujimoto, S. Nakamura, T. Toru, Chem. Commun. 2006, 2575-2577; f) M. Pohmakotr, D. Panichakul, P. Tuchinda, V. Reutrakul, Tetrahedron 2007, 63, 9429-9436; g) J. Liu, C. Ni, F. Wang, J. Hu, Tetrahedron Lett. 2008, 49, 16051608; h) G. Fourriere, J. Lalot, N. Van Hijfte, J.-C. Quirion, E. Leclerc, Tetrahedron Lett. 2009, 50, 7048-7050; i) L. Zhu, Y. Li, Y. Zhao, J. Hu, Tetrahedron Lett. 2010, 51, 6150-6152; j) J. Zhu, F. Wang, W. Huang, Y. Zhao, W. Ye, J. Hu, Synlett 2011, 899902; k) M. Obayashi, K. Kondo, Tetrahedron Lett. 1982, 23, 2327-2328.

[259] G. K. S. Prakash, J. B. Hu, G. A. Olah, J. Org. Chem. 2003, 68, 4457-4463.

[260] X. Shen, W. Zhang, C. Ni, Y. Gu, J. Hu, J. Am. Chem. Soc. 2012, 134, 16999-17002.

[261] W. M. Qiu, D. J. Burton, Tetrahedron Lett. 1996, 37, 2745-2748.

[262] K. Sato, M. Omote, A. Ando, I. Kumadaki, J. Fluorine Chem. 2004, 125, 509-515.

[263] K. Fujikawa, Y. Fujioka, A. Kobayashi, H. Amii, Org. Lett. 2011, $13,5560-5563$.

[264] Z. Feng, F. Chen, X. Zhang, Org. Lett. 2012, 14, 1938-1941.

[265] P. S. Fier, J. F. Hartwig, J. Am. Chem. Soc. 2012, 134, 5524-5527.

[266] G. K. S. Prakash, S. K. Ganesh, J.-P. Jones, A. Kulkarni, K. Masood, J. K. Swabeck, G. A. Olah, Angew. Chem. Int. Ed. 2012, 51, 12090-12094.

[267] R. P. Singh, D. Chakraborty, J. M. Shreeve, J. Fluorine Chem. 2001, 111, 153-160.

[268] A. Rivkin, K. Biswas, T. C. Chou, S. J. Danishefsky, Org. Lett. 2002, 4, 4081-4084.

[269] N. Shibata, A. Matsnev, D. Cahard, Beil. J. Org. Chem. 2010, 6, 65.

[270] P. Eisenberger, S. Gischig, A. Togni, Chem. Eur. J. 2006, 12, 2579-2586.

[271] a) T. Umemoto, S. Ishihara, Tetrahedron Lett. 1990, 31, 35793582; b) T. Umemoto, S. Ishihara, J. Am. Chem. Soc. 1993, 115, 2156-2164; c) T. Umemoto, K. Adachi, J. Org. Chem. 1994, 59, 5692-5699; d) E. Magnier, J. C. Blazejewski, M. Tordeux, C. Wakselman, Angew. Chem. Int. Ed. 2006, 45, 1279-1282. a) L. M. Yagupolskii, N. V. Kondratenko, G. N. Timofeeva, $Z h$. Org. Khim. 1984, 20, 115-118; b) Y. Mace, B. Raymondeau, C. Pradet, J.-C. Blazejewski, E. Magnier, Eur. J. Org. Chem. 2009, 1390-1397; c) J. J. Yang, R. L. Kirchmeier, J. M. Shreeve, J. Org. Chem. 1998, 63, 2656-2660.

a) I. Kieltsch, P. Eisenberger, A. Togni, Angew. Chem. Int. Ed. 2007, 46, 754-757; b) K. Stanek, R. Koller, A. Togni, J. Org. Chem. 2008, 73, 7678-7685.

[274] N. Shibata, S. Mizuta, H. Kawai, Tetrahedron: Asymmetry 2008, 19, 2633-2644.

J. M. Birchall, G. P. Irvin, R. A. Boyson, J. Chem. Soc., Perkin Trans. 2 1975, 435-439.

[276] a) H. Kimoto, S. Fujii, L. A. Cohen, J. Org. Chem. 1982, 47, 2867-2872; b) H. Kimoto, S. Fujii, L. A. Cohen, J. Org. Chem. 1984, 49, 1060-1064. 

Chem. 1986, 32, 467-470.

[278] B. R. Langlois, E. Laurent, N. Roidot, Tetrahedron Lett. 1991, 32 , 7525-7528.

[279] a) A. Studer, Angew. Chem. Int. Ed. 2012, 51, 8950-8958; b) Y. Ye, M. S. Sanford, Synlett 2012, 23, 2005-2013.

[280] M. S. Wiehn, E. V. Vinogradova, A. Togni, J. Fluorine Chem. 2010, 131, 951-957.

[281] Y. Ji, T. Brueckl, R. D. Baxter, Y. Fujiwara, I. B. Seiple, S. Su, D. G. Blackmond, P. S. Baran, Proc. Natl. Acad. Sci. USA 2011, 108, 14411-14415.

[282] D. A. Nagib, D. W. C. MacMillan, Nature 2011, 480, 224-228.

[283] T. Kino, Y. Nagase, Y. Ohtsuka, K. Yamamoto, D. Uraguchi, K. Tokuhisa, T. Yamakawa, J. Fluorine Chem. 2010, 131, 98-105.

[284] A. T. Parsons, T. D. Senecal, S. L. Buchwald, Angew. Chem. Int. Ed. 2012, 51, 2947-2950.

[285] Y. Ye, S. H. Lee, M. S. Sanford, Org. Lett. 2011, 13, 5464-5467.

[286] T. Liu, Q. Shen, Org. Lett. 2011, 13, 2342-2345.

[287] J. Xu, D.-F. Luo, B. Xiao, Z.-J. Liu, T.-J. Gong, Y. Fu, L. Liu, Chem. Commun. 2011, 47, 4300-4302.

[288] C.-P. Zhang, J. Cai, C.-B. Zhou, X.-P. Wang, X. Zheng, Y.-C. Gu, J.-C. Xiao, Chem. Commun. 2011, 47, 9516-9518.

[289] L. Chu, F.-L. Qing, Org. Lett. 2010, 12, 5060-5063.

[290] X. Jiang, L. Chu, F.-L. Qing, J. Org. Chem. 2012, 77, 1251-1257.

[291] T. D. Senecal, A. T. Parsons, S. L. Buchwald, J. Org. Chem. 2011, 76, 1174-1176.

[292] B. A. Khan, A. E. Buba, L. J. Gooßen, Chem. Eur. J. 2012, 18 , 1577-1581.

[293] P. Novák, A. Lishchynskyi, V. V. Grushin, Angew. Chem. Int. Ed. 2012, 7767-7770.

[294] Y. Ye, M. S. Sanford, J. Am. Chem. Soc. 2012, 134, 9034-9037.

[295] Y. Ye, S. A. Kuenzi, M. S. Sanford, Org. Lett. 2012, 14, 49794981.

[296] a) T. Liu, X. Shao, Y. Wu, Q. Shen, Angew. Chem. Int. Ed. 2012 51, 540-543; b) N. D. Litvinas, P. S. Fier, J. F. Hartwig, Angew. Chem. Int. Ed. 2012, 51, 536-539.

[297] L. Chu, F.-L. Qing, J. Am. Chem. Soc. 2012, 134, 1298-1304.

[298] K. Zhang, X.-L. Qiu, Y. Huang, F.-L. Qing, Eur. J. Org. Chem. 2012, 58-61.

[299] L. Chu, F.-L. Qing, J. Am. Chem. Soc. 2010, 132, 7262-7263.

[300] R. Shimizu, H. Egami, T. Nagi, J. Chae, Y. Hamashima, M. Sodeoka, Tetrahedron Lett. 2010, 51, 5947-5949.

[301] X. Mu, S. Chen, X. Zhen, G. Liu, Chem. Eur. J. 2011, 17, 60396042.

[302] E. Mejia, A. Togni, ACS Catal. 2012, 2, 521-527.

[303] a) X. Wang, L. Truesdale, J.-Q. Yu, J. Am. Chem. Soc. 2010, 132, 3648-3649; b) X.-G. Zhang, H.-X. Dai, M. Wasa, J.-Q. Yu, J. Am Chem. Soc. 2012, 134, 11948-11951.

[304] A. Hafner, S. Bräse, Angew. Chem. Int. Ed. 2012, 51, 3713-3715.

[305] N. D. Ball, J. B. Gary, Y. Ye, M. S. Sanford, J. Am. Chem. Soc. 2011, 133, 7577-7584.

[306] N. D. Ball, J. W. Kampf, M. S. Sanford, J. Am. Chem. Soc. 2010, 132, 2878-2879.

[307] D. C. Powers, E. Lee, A. Ariafard, M. S. Sanford, B. F. Yates, A. J. Canty, T. Ritter, J. Am. Chem. Soc. 2012, 134, 12002-12009.

[308] Y. Ye, N. D. Ball, J. W. Kampf, M. S. Sanford, J. Am. Chem. Soc. 2010, 132, 14682-14687.

[309] R. N. Haszeldine, J. Chem. Soc. 1949, 2856-2861.

[310] N. Kamigata, T. Fukushima, M. Yoshida, J. Chem. Soc., Chem. Commun. 1989, 1559-1560.

[311] F. Gagosz, S. Z. Zard, Org. Lett. 2003, 5, 2655-2657.

[312] L. Chu, F.-L. Qing, Chem. Commun. 2010, 46, 6285-6287.

[313] G. K. S. Prakash, M. Mandal, S. Schweizer, N. A. Petasis, G. A. Olah, Org. Lett. 2000, 2, 3173-3176.

[314] Y. Li, A. Studer, Angew. Chem. Int. Ed. 2012, 51, 8221-8224.

[315] K. Iseki, T. Nagai, Y. Kobayashi, Tetrahedron Lett. 1993, 34, 2169-2170.

[316] a) Y. Itoh, K. Mikami, Org. Lett. 2005, 7, 4883-4885; b) Y. Itoh, K. Mikami, Org. Lett. 2005, 7, 649-651.

[317] A. T. Herrmann, L. L. Smith, A. Zakarian, J. Am. Chem. Soc. 2012, 134, 6976-6979.

[318] V. Matousek, A. Togni, V. Bizet, D. Cahard, Org. Lett. 2011, 13, 5762-5765.

[319] P. V. Pham, D. A. Nagib, D. W. C. MacMillan, Angew. Chem. Int Ed. 2011, 50, 6119-6122.

[320] a) G. K. S. Prakash, F. Paknia, H. Vaghoo, G. Rasul, T. Mathew, G. A. Olah, J. Org. Chem. 2010, 75, 2219-2226; b) G. K. S.
Prakash, F. Paknia, T. Mathew, G. Mloston, J. P. Joschek, G. A Olah, Org. Lett. 2011, 13, 4128-4131.

[321] S. Noritake, N. Shibata, Y. Nomura, Y. Huang, A. Matsnev, S. Nakamura, T. Toru, D. Cahard, Org. Biomol. Chem. 2009, 7 , 3599-3604.

[322] A. E. Allen, D. W. C. MacMillan, J. Am. Chem. Soc. 2010, 132, 4986-4987.

[323] a) R. Shimizu, H. Egami, Y. Hamashima, M. Sodeoka, Angew. Chem. Int. Ed. 2012, 51, 4577-4580; b) S. Mizuta, O. GaliciaLopez, K. M. Engle, S. Verhoog, K. Wheelhouse, G. Rassias, V. Gouverneur, Chem. Eur. J. 2012, 18, 8583-8587.

[324] S. Matsubara, M. Mitani, K. Utimoto, Tetrahedron Lett. 1987, 28 , 5857-5860.

[325] a) D. B. Su, J. X. Duan, Q. Y. Chen, Tetrahedron Lett. 1991, 32, 7689-7690; b) Q. Y. Chen, J. X. Duan, J. Chem. Soc., Chem. Commun. 1993, 1389; c) J. X. Duan, D. B. Su, Q. Y. Chen, $J$. Fluorine Chem. 1993, 61, 279; d) J. Kim, J. M. Shreeve, Org. Biomol. Chem. 2004, 2, 2728; e) Y. Miyake, S.-i. Ota, Y. Nishibayashi, Chem. Eur. J. 2012, 18, 13255-13258.

[326] A. T. Parsons, S. L. Buchwald, Angew. Chem. Int. Ed. 2011, 50, 9120-9123.

[327] R. Zhu, S. L. Buchwald, J. Am. Chem. Soc. 2012, 134, 1246212465 .

[328] L. Chu, F.-L. Qing, Org. Lett. 2012, 14, 2106-2109.

[329] J. Xu, Y. Fu, D.-F. Luo, Y.-Y. Jiang, B. Xiao, Z.-J. Liu, T.-J. Gong, L. Liu, J. Am. Chem. Soc. 2011, 133, 15300-15303.

[330] X. Wang, Y. Ye, S. Zhang, J. Feng, Y. Xu, Y. Zhang, J. Wang, J. Am. Chem. Soc. 2011, 133, 16410-16413.

[331] C.-P. Zhang, Z.-L. Wang, Q.-Y. Chen, C.-T. Zhang, Y.-C. Gu, J.C. Xiao, Chem. Commun. 2011, 47, 6632-6634.

[332] X. Mu, T. Wu, H.-Y. Wang, Y.-L. Guo, G. Liu, J. Am. Chem. Soc. 2012, 134, 878-881.

[333] J. Xu, B. Xiao, C.-Q. Xie, D.-F. Luo, L. Liu, Y. Fu, Angew. Chem. Int. Ed. 2012, 12551-12554.

[334] A. Sekiya, T. Umemoto, Chem. Lett. 1982, 1519-1520.

[335] T. Umemoto, Y. Kuriu, Chem. Lett. 1982, 65-66.

[336] a) T. Umemoto, S. Ishihara, J. Fluorine Chem. 1998, 92, 181187; b) J. A. Ma, D. Cahard, J. Org. Chem. 2003, 68, 8726-8729.

[337] T. Umemoto, O. Miyano, Tetrahedron Lett. 1982, 23, 3929-3930.

[338] a) T. Umemoto, O. Miyano, J. Fluorine Chem. 1983, 22, 91-94; b) T. Umemoto, H. Tsutsumi, Bull. Chem. Soc. Jpn. 1983, 56, 631-632; c) T. Umemoto, O. Miyano, Nippon Kagaku Kaishi 1985, 2205-2207; d) T. Umemoto, A. Ando, Bull. Chem. Soc. Jpn. 1986, 59, 447-452.

[339] I. Kieltsch, P. Eisenberger, K. Stanek, A. Togni, Chimia 2008, 62, 260-263.

[340] R. Koller, Q. Huchet, P. Battaglia, J. M. Welch, A. Togni, Chem. Commun. 2009, 5993-5995.

[341] S. Noritake, N. Shibata, S. Nakamura, T. Toru, M. Shiro, Eur. J. Org. Chem. 2008, 3465-3468.

[342] W. R. Hasek, W. C. Smith, V. A. Engelhardt, J. Am. Chem. Soc. 1960, 82, 543-551.

[343] I. Popov, S. Lindeman, O. Daugulis, J. Am. Chem. Soc. 2011, 133, 9286-9289.

[344] H. S. Booth, H. M. Elsey, P. E. Burchfield, J. Am. Chem. Soc. 1935, 57, 2066-2069.

[345] Y. Kobayashi, I. Kumadaki, Tetrahedron Lett. 1969, 10, 40954096.

[346] Y. Kobayashi, I. Kumadaki, S. Sato, N. Hara, E. Chikami, Chemical \& Pharmaceutical Bulletin 1970, 18, 2334-2339.

[347] D. M. Wiemers, D. J. Burton, J. Am. Chem. Soc. 1986, 108, 832834.

[348] Y. Kobayashi, T. Yamada, A. Ohsawa, I. Kumadaki, Chemical \& Pharmaceutical Bulletin 1972, 20, 1839-1839.

[349] a) J. H. Clark, M. A. McClinton, R. J. Blade, J. Chem. Soc., Chem. Commun. 1988, 638-639; b) J. H. Clark, M. A. McClinton, C. W. Jones, P. Landon, D. Bishop, R. J. Blade, Tetrahedron Lett. 1989, 30, 2133-2136; c) J. X. Duan, D. B. Su, Q. Y. Chen, J. Fluorine Chem. 1993, 61, 279-284; d) J. X. Duan, D. B. Su, J. P. Wu, Q. Y. Chen, J. Fluorine Chem. 1994, 66, 167-169.

[350] Y. Kobayashi, K. Yamamoto, I. Kumadaki, Tetrahedron Lett. 1979, 4071-4072.

[351] a) Y. Kobayashi, I. Kumadaki, J. Chem. Soc., Perkin Trans. I 1980, 661-664; b) Y. Kobayashi, K. Yamamoto, T. Asai, M. Nakano, I. Kumadaki, J. Chem. Soc., Perkin Trans. 1 1980, 27552761; c) F. Cottet, M. Schlosser, Eur. J. Org. Chem. 2002, 327330; d) F. Cottet, M. Marull, O. Lefebvre, M. Schlosser, Eur. J. Org. Chem. 2003, 1559-1568. 
[353] a) K. Matsui, E. Tobita, M. Ando, K. Kondo, Chem. Lett. 1981 , 1719-1720; b) H. Suzuki, Y. Yoshida, A. Osuka, Chem. Lett. 1982, 135-136; c) K. A. McReynolds, R. S. Lewis, L. K. G. Ackerman, G. G. Dubinina, W. W. Brennessel, D. A. Vicic, J. Fluorine Chem. 2010, 131, 1108-1112; d) Y. Li, T. Chen, H. Wang, R. Zhang, K. Jin, X. Wang, C. Duan, Synlett 2011, 1713 1716.

[354] B. R. Langlois, N. Roques, J. Fluorine Chem. 2007, 128, 13181325.

[355] Q. Y. Chen, S. W. Wu, J. Chem. Soc., Perkin Trans. 1 1989, 2385-2387.

[356] D. B. Su, J. X. Duan, Q. Y. Chen, Tetrahedron Lett. 1991, 32, 7689-7690.

[357] N. V. Kondratenko, E. P. Vechirko, L. M. Yagupolskii, Synthesis 1980, 932-933.

[358] C.-P. Zhang, Z.-L. Wang, Q.-Y. Chen, C.-T. Zhang, Y.-C. Gu, J.C. Xiao, Angew. Chem. Int. Ed. 2011, 50, 1896-1900.

[359] J. M. Paratian, S. Sibille, J. Perichon, J. Chem. Soc., Chem. Commun. 1992, 53-54.

[360] A. Zanardi, M. A. Novikov, E. Martin, J. Benet-Buchholz, V. V. Grushin, J. Am. Chem. Soc. 2011, 133, 20901-20913.

[361] Z. Weng, R. Lee, W. Jia, Y. Yuan, W. Wang, X. Feng, K.-W. Huang, Organometallics 2011, 30, 3229-3232.

[362] I. Ruppert, K. Schlich, W. Volbach, Tetrahedron Lett. 1984, 25, 2195-2198.

[363] H. Urata, T. Fuchikami, Tetrahedron Lett. 1991, 32, 91-94.

[364] a) G. G. Dubinina, H. Furutachi, D. A. Vicic, J. Am. Chem. Soc. 2008, 130, 8600-8601; b) G. G. Dubinina, J. Ogikubo, D. A. Vicic, Organometallics 2008, 27, 6233-6235.

[365] M. Oishi, H. Kondo, H. Amii, Chem. Commun. 2009, 1909-1911.

[366] H. Morimoto, T. Tsubogo, N. D. Litvinas, J. F. Hartwig, Angew. Chem. Int. Ed. 2011, 50, 3793-3798.

[367] O. A. Tomashenko, E. C. Escudero-Adan, M. Martinez Belmonte, V. V. Grushin, Angew. Chem. Int. Ed. 2011, 50, 7655-7659.

[368] T. Knauber, F. Arikan, G.-V. Roeschenthaler, L. J. Gooßen, Chem. Eur. J. 2011, 17, 2689-2697.

[369] H. Kondo, M. Oishi, K. Fujikawa, H. Amii, Adv. Synth. Catal. 2011, 353, 1247-1252.

[370] a) T. Billard, B. R. Langlois, G. Blond, Tetrahedron Lett. 2000 , 41,8777-8780; b) T. Billard, S. Bruns, B. R. Langlois, Org. Lett. 2000, 2, 2101-2103.

[371] T. Allmendinger, R. W. Lang, Tetrahedron Lett. 1991, 32, 339340 .

[372] a) X. G. Zhang, F. L. Qing, Y. G. Yang, J. Yu, X. K. Fu, Tetrahedron Lett. 2000, 41, 2953-2955; b) F. L. Qing, X. G. Zhang, Tetrahedron Lett. 2001, 42, 5929-5931; c) X. G. Zhang, F. L. Qing, Y. Y. Peng, J. Fluorine Chem. 2001, 108, 79-82; d) F. L. Qing, X. G. Zhang, Y. Y. Peng, J. Fluorine Chem. 2001, 111, 185-187.

[373] I. Nowak, M. J. Robins, J. Org. Chem. 2007, 72, 2678-2681.

[374] A. Hafner, S. Braese, Adv. Synth. Catal. 2011, 353, 3044-3048.

[375] a) F. Ozawa, K. Kurihara, M. Fujimori, T. Hidaka, T. Toyoshima, A. Yamamoto, Organometallics 1989, 8, 180-188; b) J. M. Brown, P. J. Guiry, Inorg. Chim. Acta 1994, 220, 249-259.

[376] a) V. V. Grushin, W. J. Marshall, J. Am. Chem. Soc. 2006, 128, 12644-12645; b) V. I. Bakhmutov, F. Bozoglian, K. Gomez, G. Gonzalez, V. V. Grushin, S. A. Macgregor, E. Martin, F. M. Miloserdov, M. A. Novikov, J. A. Panetier, L. V. Romashov, Organometallics 2012, 31, 1315-1328.

[377] E. J. Cho, T. D. Senecal, T. Kinzel, Y. Zhang, D. A. Watson, S. L. Buchwald, Science 2010, 328, 1679-1681.

[378] E. J. Cho, S. L. Buchwald, Org. Lett. 2011, 13, 6552-6555.

[379] G. K. S. Prakash, H. S. Krishnan, P. V. Jog, A. P. Iyer, G. A. Olah, Org. Lett. 2012, 14, 1146-1149.

[380] P. G. Janson, I. Ghoneim, N. O. Ilchenko, K. J. Szabó, Org. Lett. 2012, 14, 2882-2885.

[381] M. Omote, M. Tanaka, A. Ikeda, S. Nomura, A. Tarui, K. Sato, A. Ando, Org. Lett. 2012, 14, 2286-2289.

[382] a) H. J. Emeleus, R. N. Haszeldine, J. Chem. Soc. 1949, 2948 2952; b) H. J. Emeleus, R. N. Haszeldine, J. Chem. Soc. 1949, 2953-2956.

[383] a) T. Kitazume, N. Ishikawa, Chem. Lett. 1981, 1679-1680; b) M. M. Kremlev, W. Tyrra, A. I. Mushta, D. Naumann, Y. L. Yagupolskii, J. Fluorine Chem. 2010, 131, 212-216.

[384] D. J. Burton, Z. Y. Yang, K. J. Macneil, J. Fluorine Chem. 1991, $52,251-255$.
[385] M. Fujita, M. Obayashi, T. Hiyama, Tetrahedron 1988, 44, 41354145.

[386] J. Wiedemann, T. Heiner, G. Mloston, G. K. S. Prakash, G. A. Olah, Angew. Chem. Int. Ed. 1998, 37, 820-821.

[387] a) G. K. S. Prakash, M. Mandal, G. A. Olah, Org. Lett. 2001, 3, 2847-2850; b) G. K. S. Prakash, M. Mandal, G. A. Olah, Angew. Chem. Int. Ed. 2001, 40, 589-590; c) Y. Kawano, T. Mukaiyama, Chem. Lett. 2005, 34, 894-895.

[388] G. K. S. Prakash, M. Mandal, J. Am. Chem. Soc. 2002, 124, 65386539.

[389] a) G. K. S. Prakash, M. Mandal, G. A. Olah, Synlett 2001, 77-78; b) Y. Kawano, H. Fujisawa, T. Mukaiyama, Chem. Lett. 2005, 34 422-423.

[390] R. P. Singh, J. M. Leitch, B. Twamley, J. M. Shreeve, J. Org. Chem. 2001, 66, 1436-1440.

[391] D. V. Sevenard, V. Y. Sosnovskikh, A. A. Kolomeitsev, M. H. Konigsmann, G. V. Roschenthaler, Tetrahedron Lett. 2003, 44, 7623-7627.

[392] a) G. K. S. Prakash, R. Mogi, G. A. Olah, Org. Lett. 2006, 8, 3589-3592; b) V. V. Levin, M. A. Kozlov, Y.-H. Song, A. D. Dilman, P. A. Belyakov, M. I. Struchkova, V. A. Tartakovsky, Tetrahedron Lett. 2008, 49, 3108-3111; c) R. T. Gritsenko, V. V. Levin, A. D. Dilman, P. A. Belyakov, M. I. Struchkova, V. A. Tartakovsky, Tetrahedron Lett. 2009, 50, 2994-2997.

[393] H. Kawai, K. Tachi, E. Tokunaga, M. Shiro, N. Shibata, Angew. Chem. Int. Ed. 2011, 50, 7803-7806.

[394] a) T. Hagiwara, T. Kobayashi, T. Fuchikami, Main Group Chem. 1997, 2, 13-15; b) G. K. S. Prakash, C. Panja, H. Vaghoo, V. Surampudi, R. Kultyshev, M. Mandal, G. Rasul, T. Mathew, G. A. Olah, J. Org. Chem. 2006, 71, 6806-6813; c) L. Bernardi, E. Indrigo, S. Pollicino, A. Ricci, Chem. Commun. 2012, 48, 14281430.

[395] S. Mizuta, N. Shibata, T. Sato, H. Fujimoto, S. Nakamura, T. Toru, Synlett 2006, 267-270.

[396] S. Roussel, T. Billard, B. R. Langlois, L. Saint-James, Chem. Eur. J. 2005, 11, 939-944.

[397] H. Sugimoto, S. Nakamura, Y. Shibata, N. Shibata, T. Toru, Tetrahedron Lett. 2006, 47, 1337-1340.

[398] a) K. Iseki, T. Nagai, Y. Kobayashi, Tetrahedron Lett. 1994, 35, 3137-3138; b) S. Caron, N. M. Do, P. Arpin, A. Larivee, Synthesis 2003, 1693-1698.

[399] S. Mizuta, N. Shibata, S. Akiti, H. Fujimoto, S. Nakamura, T. Toru, Org. Lett. 2007, 9, 3707-3710.

[400] H. Kawai, K. Tachi, E. Tokunaga, M. Shiro, N. Shibata, Org. Lett. 2010, 12, 5104-5107.

[401] S. Mizuta, N. Shibata, M. Hibino, S. Nagano, S. Nakamura, T. Toru, Tetrahedron 2007, 63, 8521-8528.

[402] a) H. Nagao, Y. Kawano, T. Mukaiyama, Bull. Chem. Soc. Jpn. 2007, 80, 2406-2412; b) H. Nagao, Y. Yamane, T. Mukaiyama, Chem. Lett. 2007, 36, 666-667.

[403] H. Kawai, A. Kusuda, S. Nakamura, M. Shiro, N. Shibata, Angew. Chem. Int. Ed. 2009, 48, 6324-6327.

[404] T. Billard, B. R. Langlois, G. Blond, Eur. J. Org. Chem. 2001, 1467-1471.

[405] V. V. Levin, A. D. Dilman, P. A. Belyakov, M. I. Struchkova, V. A. Tartakovsky, Tetrahedron Lett. 2011, 52, 281-284.

[406] a) G. Pawelke, J. Fluorine Chem. 1989, 42, 429-433; b) W. Xu, W. R. Dolbier, J. Org. Chem. 2005, 70, 4741-4745; c) M. Medebielle, W. R. Dolbier, Jr., J. Fluorine Chem. 2008, 129, 930942.

[407] Q. Y. Chen, S. W. Wu, J. Chem. Soc., Chem. Commun. 1989, 705-706.

[408] J. G. Macneil, D. J. Burton, J. Fluorine Chem. 1991, 55, 225-227.

[409] Q. Y. Chen, J. X. Duan, Tetrahedron Lett. 1993, 34, 4241-4244.

[410] J. M. Paratian, E. Labbe, S. Sibille, J. Perichon, J. Organomet. Chem. 1995, 489, 137-143.

[411] Y. Chang, C. Cai, Tetrahedron Lett. 2005, 46, 3161-3164.

[412] T. S. N. Zhao, K. J. Szabo, Org. Lett. 2012, 14, 3966-3969.

[413] M. Hu, C. Ni, J. Hu, J. Am. Chem. Soc. 2012, 134, 15257-15260.

[414] T. Furukawa, T. Nishimine, E. Tokunaga, K. Hasegawa, M. Shiro, N. Shibata, Org. Lett. 2011, 13, 3972-3975.

[415] Y. Li, F. Liang, Q. Li, Y.-c. Xu, Q.-R. Wang, L. Jiang, Org. Lett. 2011, 13, 6082-6085.

[416] a) M. Steinman, J. G. Topliss, R. Alekel, Y. S. Wong, E. E. York, J. Med. Chem. 1973, 16, 1354-1360; b) E. H. Banitt, W. E. Coyne, J. R. Schmid, A. Mendel, J. Med. Chem. 1975, 18, 1130-1134; c) E. H. Banitt, W. R. Bronn, W. E. Coyne, J. R. Schmid, J. Med. Chem. 1977, 20, 821-826; d) J. R. Wetterau, R. E. Gregg, T. W. 
Harrity, C. Arbeeny, M. Cap, F. Connolly, C. H. Chu, R. J. George, D. A. Gordon, H. Jamil, K. G. Jolibois, L. K. Kunselman, S. J. Lan, T. J. Maccagnan, B. Ricci, M. J. Yan, D. Young, Y. Chen, O. M. Fryszman, J. V. H. Logan, C. L. Musial, M. A. Poss, J. A. Robl, L. M. Simpkins, W. A. Slusarchyk, R. Sulsky, P. Taunk, D. R. Magnin, J. A. Tino, R. M. Lawrence, J. K. Dickson, S. A. Biller, Science 1998, 282, 751-754; e) J. A. Robl, R. Sulsky, C. Q. Sun, L. M. Simpkins, T. Wang, J. K. Dickson, Y. Chen, D. R. Magnin, P. Taunk, W. A. Slusarchyk, S. A. Biller, S. J. Lan, F. Connolly, L. K. Kunselman, T. Sabrah, D. Jamil, D. Gordon, T. W. Harrity, J. R. Wetterau, J. Med. Chem. 2001, 44, 851-856. a) V. C. R. McLoughlin, J. Thrower, Tetrahedron 1969, 25, 5921 5940; b) Y. Kobayashi, K. Yamamoto, I. Kumadaki, Tetrahedron Lett. 1979, 20, 4071-4072; c) N. V. Kondratenko, E. P. Vechirko, L. M. Yagupolskii, Synthesis 1980, 1980, 932-933; d) H. Urata, T Fuchikami, Tetrahedron Lett. 1991, 32, 91-94; e) Q.-Y. Chen, J.X. Duan, J. Chem. Soc., Chem. Commun. 1993, 1389-1391; f) J. X. Duan, Q. Y. Chen, J. Chem. Soc., Perkin Trans. 1 1994, $725-$ $730 ;$ g) J. M. Paratian, E. Labbé, S. Sibille, J. Périchon, $J$ Organomet. Chem. 1995, 489, 137-143; h) J. Kim, J. n. M. Shreeve, Org. Biomol. Chem. 2004, 2, 2728-2734.

[418] H. Kawai, T. Furukawa, Y. Nomura, E. Tokunaga, N. Shibata, Org. Lett. 2011, 13, 3596-3599.

[419] D. A. Culkin, J. F. Hartwig, Organometallics 2004, 23, 33983416.

[420] Y. Zhao, J. Hu, Angew. Chem. Int. Ed. 2012, 51, 1033-1036.

[421] M. Fujita, T. Morita, T. Hiyama, Tetrahedron Lett. 1986, 27, 2135-2138.

[422] a) M. Vanderpuy, J. Fluorine Chem. 1993, 61, 133-140; b) Z. Y. Long, Q. Y. Chen, Tetrahedron Lett. 1998, 39, 8487-8490.

[423] a) T. Umemoto, Y. Gotoh, J. Fluorine Chem. 1985, 28, 235-239; b) T. Umemoto, Y. Gotoh, J. Fluorine Chem. 1986, 31, 231-236; c) J. Zhang, G. R. Martin, D. D. DesMarteau, Chem. Commun. 2003, 2334-2335.

[424] a) T. Umemoto, Y. Gotoh, Bull. Chem. Soc. Jpn. 1991, 64, 20082010; b) V. Montanari, G. Resnati, Tetrahedron Lett. 1994, 35, 8015-8018; c) T. Umemoto, Y. Gotoh, Bull. Chem. Soc. Jpn. 1987, 60, 3307-3313

[425] T. Umemoto, Y. Gotoh, Bull. Chem. Soc. Jpn. 1987, 60, 38233825.

[426] a) M. LeBlanc, G. Santini, J. Guion, J. G. Riess, Tetrahedron 1973, 29, 3195-3201; b) G. Santini, M. Leblanc, J. G. Riess, Tetrahedron 1973, 29, 2411-2414; c) P. L. Coe, N. E. Milner, J. A. Smith, J. Chem. Soc., Perkin Trans. 1 1975, 654-656; d) G. J. Chen, C. Tamborski, J. Fluorine Chem. 1989, 43, 207-228.

[427] J. C. Xiao, C. F. Ye, J. M. Shreeve, Org. Lett. 2005, 7, 1963-1965.

[428] T. Kitazume, N. Ishikawa, Chem. Lett. 1982, 137-140.

[429] G. E. Carr, R. D. Chambers, T. F. Holmes, D. G. Parker, J. Chem. Soc., Perkin Trans. 1 1988, 921-926.

[430] R. N. Loy, M. S. Sanford, Org. Lett. 2011, 13, 2548-2551.

[431] L. F. Chen, J. Mohtasham, G. L. Gard, J. Fluorine Chem. 1989, 43, 329-347.

[432] N. Kamigata, T. Fukushima, Y. Terakawa, M. Yoshida, H. Sawada, J. Chem. Soc., Perkin Trans. 1 1991, 627-633.

[433] T. Kitazume, N. Ishikawa, Chem. Lett. 1982, 1453-1454.

[434] T. Kitazume, N. Ishikawa, J. Am. Chem. Soc. 1985, 107, 51865191.

[435] D. J. Burton, G. A. Hartgraves, J. Hsu, Tetrahedron Lett. 1990, 31, 3699-3702

[436] a) O. R. Pierce, E. T. McBee, G. F. Judd, J. Am. Chem. Soc. 1954, 76, 474-478; b) P. Johncock, J. Organomet. Chem. 1969, 19, 257 265; c) P. G. Gassman, N. J. Oreilly, Tetrahedron Lett. 1985, 26, 5243-5246; d) P. G. Gassman, N. J. Oreilly, J. Org. Chem. 1987, 52, 2481-2490.

[437] a) E. T. McBee, C. W. Roberts, A. F. Meiners, J. Am. Chem. Soc. 1957, 79, 335-337; b) E. T. McBee, H. P. Braendlin, R. D. Battershell, J. Org. Chem. 1963, 28, 1131-1133; c) D. D. Denson, C. F. Smith, Tamborsk.C, J. Fluorine Chem. 1974, 3, 247-258; d) C. F. Smith, E. J. Soloski, Tamborsk.C, J. Fluorine Chem. 1974, 4, 35-45; e) N. Thoai, J. Fluorine Chem. 1975, 5, 115-125; f) K. V. Werner, A. Gisser, J. Fluorine Chem. 1977, 10, 387-394

[438] a) G. Santini, M. Leblanc, J. G. Riess, J. Chem. Soc., Chem Commun. 1975, 678-679; b) G. Santini, M. Leblanc, J. G. Riess, J. Organomet. Chem. 1977, 140, 1-9.

[439] T. Kitazume, N. Ishikawa, Chem. Lett. 1981, 1337-1338.

[440] R. Krishnamurti, D. R. Bellew, G. K. S. Prakash, J. Org. Chem. 1991, 56, 984-989. 520.

[443] G. K. S. Prakash, Y. Wang, R. Mogi, J. Hu, T. Mathew, G. A. Olah, Org. Lett. 2010, 12, 2932-2935.

[444] L. M. Yagupolskii, Maletina, II, N. V. Kondratenko, V. V. Orda, Synthesis 1978, 835-837.

[445] T. Umemoto, Y. Kuriu, Tetrahedron Lett. 1981, 22, 5197-5200.

[446] T. Umemoto, Y. Kuriu, H. Shuyama, Chem. Lett. 1981, 16631666.

[447] T. Umemoto, O. Miyano, Bull. Chem. Soc. Jpn. 1984, 57, 33613362.

[448] T. Umemoto, Y. Kuriu, O. Miyano, Tetrahedron Lett. 1982, 23, 3579-3582.

[449] T. Umemoto, Y. Kuriu, S. Nakayama, Tetrahedron Lett. 1982, 23 , 4101-4102.

[450] T. Umemoto, Y. Kuriu, S. Nakayama, Tetrahedron Lett. 1982, 23 , 1169-1172.

[451] K. Miura, M. Taniguchi, K. Nozaki, K. Oshima, K. Utimoto, Tetrahedron Lett. 1990, 31, 6391-6394.

[452] a) I. Rico, C. Wakselman, J. Fluorine Chem. 1982, 20, 759-764; b) I. Rico, C. Wakselman, J. Fluorine Chem. 1982, 20, 765-770 M. T. Kolycheva, Gerus, II, V. P. Kukhar, Amino Acids 1993, 5 , 99-101.

[454] T. Takagi, M. Tamura, M. Shibakami, H. D. Quan, A. Sekiya, J. Fluorine Chem. 2000, 101, 15-17.

[455] S. Altomonte, M. Zanda, J. Fluorine Chem. 2012, 143, 57-93.

[456] F. Leroux, P. Jeschke, M. Schlosser, Chem. Rev. 2005, 105, 827856.

[457] P. Jeschke, E. Baston, F. R. Leroux, Mini-Rev. Med. Chem. 2007, 7, 1027-1034.

[458] W. Dmowski, M. Kamiński, J. Fluorine Chem. 1983, 23, 219-228.

[459] T. Umemoto, K. Adachi, S. Ishihara, J. Org. Chem. 2007, 72, 6905-6917.

[460] a) R. Koller, K. Stanek, D. Stolz, R. Aardoom, K. Niedermann, A. Togni, Angew. Chem. Int. Ed. 2009, 48, 4332-4336; b) S. Fantasia, J. M. Welch, A. Togni, J. Org. Chem. 2010, 75, 1779-1782.

[461] a) L. M. Yagupolskii, Dokl. Akad. Nauk S.S.S.R. 1955, 105, 100102; b) N. N. Yarovenko, A. S. Vasileva, Zh. Obshch. Khim. 1958, 28, 2502-2504; c) L. M. Yagupolskii, V. I. Troitskaya, $Z h$. Obshch. Khim. 1961, 31, 915; d) R. Louw, P. W. Franken, Chem. Ind. (London) 1977, 127-128; e) A. E. Feiring, J. Org. Chem. 1979, 44, 2907-2910; f) L. M. Yagupolskii, V. V. Orda, Zh. Obshch. Khim. 1964, 34, 1979-1984; g) J. Salome, C. Mauger, S. Brunet, V. Schanen, J. Fluorine Chem. 2004, 125, 1947-1950.

[462] F. Mathey, J. Bensoam, Tetrahedron Lett. 1973, 14, 2253-2256.

[463] W. A. Sheppard, J. Org. Chem. 1964, 29, 1-11.

[464] a) M. Kuroboshi, K. Suzuki, T. Hiyama, Tetrahedron Lett. 1992 , 33, 4173-4176; b) I. Ben-David, D. Rechavi, E. Mishani, S. Rozen, J. Fluorine Chem. 1999, 97, 75-78; c) K. Kanie, Y. Tanaka, K. Suzuki, M. Kuroboshi, T. Hiyama, Bull. Chem. Soc. Jpn. 2000, 73, 471-484.

[465] B. Farbwerke Hoechst, n. Pat, Chem. Abstr. 1957, 51, 14803.

[466] L. M. Yagupolskii, V. F. Bystrov, A. U. Stepanyants, Y. A. Fialkov, Zh. Obshch. Khim. 1964, 34, 3682.

[467] B. Manteau, P. Genix, L. Brelot, J.-P. Vors, S. Pazenok, F Giornal, C. Leuenberger, F. R. Leroux, Eur. J. Org. Chem. 2010, 6043-6066.

[468] A. A. Kolomeitsev, M. Vorobyev, H. Gillandt, Tetrahedron Lett. 2008, 49, 449-454

[469] C. Huang, T. Liang, S. Harada, E. Lee, T. Ritter, J. Am. Chem Soc. 2011, 133, 13308-13310.

[470] C.-P. Zhang, D. A. Vicic, Organometallics 2012, 31, 7812-7815.

[471] F. Venturini, W. Navarrini, A. Famulari, M. Sansotera, P. Dardani, V. Tortelli, J. Fluorine Chem. 2012, 140, 43-48.

[472] N. Santschi, A. Togni, J. Org. Chem. 2011, 76, 4189-4193.

[473] S. Capone, I. Kieltsch, O. Floegel, G. Lelais, A. Togni, D. Seebach, Helv. Chim. Acta 2008, 91, 2035-2056.

[474] T. Billard, N. Roques, B. R. Langlois, J. Org. Chem. 1999, 64, 3813-3820.

[475] T. Billard, B. R. Langlois, Tetrahedron Lett. 1996, 37, 6865-6868.

[476] C. Pooput, M. Medebielle, W. R. Dolbier, Org. Lett. 2004, 6, 301-303.

[477] B. Langlois, D. Montegre, N. Roidot, J. Fluorine Chem. 1994, 68, 63-66.

[478] F. Baert, J. Colomb, T. Billard, Angew. Chem. Int. Ed. 2012, 51, 10382-10385. 
[479] D. J. Adams, J. H. Clark, J. Org. Chem. 2000, 65, 1456-1460.

[480] D. J. Adams, A. Goddard, J. H. Clark, D. J. Macquarrie, Chem. Commun. 2000, 987-988.

[481] J. H. Clark, C. W. Jones, A. P. Kybett, M. A. McClinton, J. M. Miller, D. Bishop, R. J. Blade, J. Fluorine Chem. 1990, 48, 249255.

[482] Q. Y. Chen, J. X. Duan, J. Chem. Soc., Chem. Commun. 1993, 918-919.

[483] G. Teverovskiy, D. S. Surry, S. L. Buchwald, Angew. Chem. Int Ed. 2011, 50, 7312-7314.

[484] C.-P. Zhang, D. A. Vicic, J. Am. Chem. Soc. 2012, 134, 183-185.

[485] C.-P. Zhang, D. A. Vicic, Chem. Asian. J. 2012, 7, 1756-1758.

[486] C. Chen, Y. Xie, L. Chu, R.-W. Wang, X. Zhang, F.-L. Qing, Angew. Chem. Int. Ed. 2012, 51, 2492-2495.

[487] C. Chen, L. Chu, F.-L. Qing, J. Am. Chem. Soc. 2012, 134, 12454-12457.

[488] L. D. Tran, I. Popov, O. Daugulis, J. Am. Chem. Soc. 2012, 134, 18237-18240.

[489] K. Niedermann, N. Früh, R. Senn, B. Czarniecki, R. Verel, A. Togni, Angew. Chem. Int. Ed. 2012, 6511-6515.

[490] W. Tyrra, J. Fluorine Chem. 2001, 109, 189-194.

[491] M. Kuroboshi, T. Hiyama, Tetrahedron Lett. 1992, 33, 41774178.

[492] W. Dmowski, M. Kaminski, J. Fluorine Chem. 1983, 23, 207-218.

[493] M. Kuroboshi, T. Hiyama, Tetrahedron Lett. 1994, 35, 39833984.

[494] M. Kuroboshi, K. Mizuno, K. Kanie, T. Hiyama, Tetrahedron Lett. 1995, 36, 563-566.

[495] K. Niedermann, N. Frueh, E. Vinogradova, M. S. Wiehn, A. Moreno, A. Togni, Angew. Chem. Int. Ed. 2011, 50, 1059-1063.

[496] N. Armanino, R. Koller, A. Togni, Organometallics 2010, 29, 1771-1777.

[497] a) P. A. Bartlett, A. Otake, J. Org. Chem. 1995, 60, 3107-3111; b) R. J. Abraham, S. L. R. Ellison, P. Schonholzer, W. A. Thomas, Tetrahedron 1986, 42, 2101-2110.

[498] a) J. F. Lontz, W. B. Happoldt, Ind. Eng. Chem. 1952, 44, 18001805; b) K. L. Berry, J. H. Peterson, J. Am. Chem. Soc. 1951, 73, 5195-5197.

[499] a) T. Allmendinger, E. Felder, E. Hungerbuhler, Tetrahedron Lett. 1990, 31, 7301-7304; b) T. Allmendinger, P. Furet, E. Hungerbuhler, Tetrahedron Lett. 1990, 31, 7297-7300; c) L. G. Boros, B. Decorte, R. H. Gimi, J. T. Welch, Y. Wu, R. E. Handschumacher, Tetrahedron Lett. 1994, 35, 6033-6036.

[500] J. T. Welch, J. Lin, Tetrahedron 1996, 52, 291-304.

[501] a) M. Schlosser, M. Zimmermann, Synthesis 1969, 75-76; b) S. Hayashi, T. Nakai, N. Ishikawa, D. J. Burton, D. G. Naae, H. S. Kesling, Chem. Lett. 1979, 983-986; c) D. A. Dixon, B. E. Smart, J. Am. Chem. Soc. 1986, 108, 7172-7177.

[502] J. T. Welch, R. W. Herbert, J. Org. Chem. 1990, 55, 4782-4784.

[503] a) G. K. S. Prakash, A. Shakhmin, M. Zibinsky, I. Ledneczki, S. Chacko, G. A. Olah, J. Fluorine Chem. 2010, 131, 1192-1197; b) L. Zhu, C. Ni, Y. Zhao, J. Hu, Tetrahedron 2010, 66, 5089-5100.

[504] S. K. Mandal, A. K. Ghosh, R. Kumar, B. Zajc, Org. Biomol. Chem. 2012, 10, 3164-3167.

[505] T. Kitazume, N. Ishikawa, Chem. Lett. 1981, 1259-1260.

[506] W. Zhang, W. Huang, J. Hu, Angew. Chem. Int. Ed. 2009, 48, 9858-9861.

[507] P. Albert, J. Cousseau, J. Chem. Soc., Chem. Commun. 1985, 961-962.

[508] A. Gorgues, D. Stephan, J. Cousseau, J. Chem. Soc., Chem. Commun. 1989, 1493-1494.

[509] J. A. Akana, K. X. Bhattacharyya, P. Mueller, J. P. Sadighi, J. Am. Chem. Soc. 2007, 129, 7736-7737.

[510] B. C. Gorske, C. T. Mbofana, S. J. Miller, Org. Lett. 2009, 11, 4318-4321.

[511] M. Schuler, F. Silva, C. Bobbio, A. Tessier, V. Gouverneur, Angew. Chem. Int. Ed. 2008, 47, 7927-7930.

[512] T. de Haro, C. Nevado, Chem. Commun. 2011, 47, 248-249.

[513] a) Y. Nakamura, M. Okada, M. Koura, M. Tojo, A. Saito, A. Sato, T. Taguchi, J. Fluorine Chem. 2006, 127, 627-636; b) T. Narumi, A. Niida, K. Tomita, S. Oishi, A. Otaka, H. Ohno, N. Fujii, Chem Commun. 2006, 4720-4722; c) T. Narumi, K. Tomita, E. Inokuchi, K. Kobayashi, S. Oishi, H. Ohno, N. Fujii, Tetrahedron 2008, 64, $4332-4346$.

[514] M. Bergeron, T. Johnson, J.-F. Paquin, Angew. Chem. Int. Ed. 2011, 50, 11112-11116.

[515] J.-F. Paquin, Synlett 2011, 289-293.

[516] S. H. Lee, J. Schwartz, J. Am. Chem. Soc. 1986, 108, 2445-2447.
[517] M. Kang, S.-A. Lee, N. Kang, B. Moon, Bull. Korean Chem. Soc. 2011, 32, 3022-3030

[518] Z. Y. Yang, D. J. Burton, Tetrahedron Lett. 1990, 31, 1369-1372.

[519] H. Zhang, C.-B. Zhou, Q.-Y. Chen, J.-C. Xiao, R. Hong, Org. Lett. 2011, 13, 560-563.

[520] V. N. Korotchenko, A. V. Shastin, V. G. Nenajdenko, E. S. Balenkova, Tetrahedron 2001, 57, 7519-7527.

[521] a) S. A. Fuqua, W. G. Duncan, R. M. Silverstein, J. Org. Chem. 1965, 30, 1027-1029; b) S. A. Fuqua, W. G. Duncan, R. M. Silverstein, J. Org. Chem. 1965, 30, 2543-2545; c) D. G. Naae, D. J. Burton, J. Fluorine Chem. 1971, 1, 123-125; d) D. G. Naae, D. J. Burton, Synth. Commun. 1973, 3, 197-200; e) G. A. Wheaton, D. J. Burton, J. Org. Chem. 1983, 48, 917-927; f) P. Jubault, C. Feasson, N. Collignon, Bull. Soc. Chim. Fr. 1995, 132, 850-856.

[522] S. R. Piettre, L. Cabanas, Tetrahedron Lett. 1996, 37, 5881-5884.

[523] I. Nowak, M. J. Robins, Org. Lett. 2005, 7, 721-724.

[524] V. G. Nenaidenko, G. N. Varseev, V. N. Korotchenko, A. V. Shastin, E. S. Balenkova, J. Fluorine Chem. 2003, 124, 115-118. M. Pohmakotr, K. Boonkitpattarakul, W. Ieawsuwan, S Jarussophon, N. Duangdee, P. Tuchinda, V. Reutrakul, Tetrahedron 2006, 62, 5973-5985.

[526] a) J. S. Sabol, J. R. McCarthy, Tetrahedron Lett. 1992, 33, 31013104; b) G. K. S. Prakash, J. B. Hu, W. Ying, G. A. Olah, Angew. Chem. Int. Ed. 2004, 43, 5203-5206.

[527] J. Ichikawa, Y. Ishibashi, H. Fukui, Tetrahedron Lett. 2003, 44, $707-710$.

[528] a) J. P. Begue, D. Bonnetdelpon, M. H. Rock, Synlett 1995, 659 660 ; b) J. P. Begue, D. BonnetDelpon, M. H. Rock, J. Chem. Soc. Perkin Trans. 1 1996, 1409-1413.

[529] J. P. Begue, D. Bonnetdelpon, M. H. Rock, Tetrahedron Lett. 1995, 36, 5003-5006.

[530] K. Fuchibe, M. Takahashi, J. Ichikawa, Angew. Chem. Int. Ed. 2012, 12059-12062.

[531] J. P. Begue, D. Bonnetdelpon, J. M. Percy, M. H. Rock, R. D. Wilkes, J. Chem. Soc., Chem. Commun. 1995, 1857-1857.

[532] T. Miura, Y. Ito, M. Murakami, Chem. Lett. 2008, 37, 1006-1007.

[533] P. A. Morken, D. J. Burton, J. Org. Chem. 1993, 58, 1167-1172.

[534] G. A. DeBoos, J. J. Fullbrook, W. M. Owton, J. M. Percy, A. C. Thomas, Synlett 2000, 963-966.

[535] M. Kuroboshi, N. Yamada, Y. Takebe, T. Hiyama, Tetrahedron Lett. 1995, 36, 6271-6274.

[536] S. Martin, R. Sauvetre, J. F. Normant, J. Organomet. Chem. 1984 264, 155-161.

[537] C. R. Davis, D. J. Burton, Tetrahedron Lett. 1996, 37, 7237-7240.

[538] R. P. Singh, U. Majumder, J. M. Shreeve, J. Org. Chem. 2001, 66, 6263-6267.

[539] a) J. Bergman, O. Solin, Nucl. Med. Biol. 1997, 24, 677-683; b) H. Teare, E. G. Robins, A. Kirjavainen, S. Forsback, G. Sandford, O. Solin, S. K. Luthra, V. Gouverneur, Angew. Chem. Int. Ed. 2010, 49, 6821-6824.

[540] K. Hamacher, H. H. Coenen, G. Stocklin, J. Nucl. Med. 1986, 27, 235-238.

[541] a) M. Attina, F. Cacace, A. P. Wolf, J. Chem. Soc., Chem. Commun. 1983, 108-109; b) G. Angelini, M. Speranza, A. P. Wolf, C. Y. Shiue, J. Fluorine Chem. 1985, 27, 177-191.

[542] Y. S. Ding, S. J. Gatley, J. S. Fowler, N. D. Volkow, D. Aggarwal, J. Logan, S. L. Dewey, F. Liang, F. I. Carroll, M. J. Kuhar, Synapse 1996, 24, 403-407.

[543] a) V. W. Pike, F. I. Aigbirhio, J. Chem. Soc., Chem. Commun. 1995, 2215-2216; b) T. L. Ross, J. Ermert, C. Hocke, H. H. Coenen, J. Am. Chem. Soc. 2007, 129, 8018-8025.

[544] Y. Yamada, M. Okawara, Bull. Chem. Soc. Jpn. 1972, 45, 1860 1863.

[545] E. A. Merritt, B. Olofsson, Angew. Chem. Int. Ed. 2009, 48 , 9052-9070.

[546] a) R. Chirakal, G. Firnau, J. Couse, E. S. Garnett, Int. J. Appl. Radiat. Isot. 1984, 35, 651-653; b) M. Namavari, A. Bishop, N. Satyamurthy, G. Bida, J. R. Barrio, Appl. Radiat. Isot. 1992, 43, 989-996.

[547] a) H. D. Frame, J. L. Huston, I. Sheft, Inorg. Chem. 1969, 8, 1549-1550; b) G. Firnau, R. Chirakal, S. Sood, E. S. Garnett, J. Labelled Compd. Radiopharm. 1981, 18, 7-8; c) C. Y. Shiue, K. C. To, A. P. Wolf, J. Labelled Compd. Radiopharm. 1983, 20, 157-162; d) F. I. Aigbirhio, V. W. Pike, R. G. Smith, C. A. Ramsden, J. Nucl. Med. 1998, 39, 231P-232P; e) N. Vasdev, B. E. Pointner, R. Chirakal, G. J. Schrobilgen, J. Am. Chem. Soc. 2002, 124, 12863-12868. 
[548] a) N. Satyamurthy, G. T. Bida, M. E. Phelps, J. R. Barrio, Appl. Radiat. Isot. 1990, 41, 733-738; b) H. Teare, E. G. Robins, E. Arstad, S. K. Luthra, V. Gouverneur, Chem. Commun. 2007, 2330-2332.

[549] F. Oberdorfer, E. Hofmann, W. Maier-Borst, J. Labelled Compd. Radiopharm. 1988, 35, 999-1006.

[550] T. Ido, C. N. Wan, V. Casella, J. S. Fowler, A. P. Wolf, M. Reivich, D. E. Kuhl, J. Labelled Compd. Radiopharm. 1978, 14 , 175-183.

[551] G. Firnau, E. S. Garnett, R. Chirakal, S. Sood, C. Nahmias, G. Schrobilgen, Appl. Radiat. Isot. 1986, 37, 669-675.

[552] Z. Gao, Y. H. Lim, M. Tredwell, L. Li, S. Verhoog, M. Hopkinson, W. Kaluza, T. L. Collier, J. Passchier, M. Huiban, V. Gouverneur, Angew. Chem. Int. Ed. 2012, 6733-6737.

[553] E. Lee, A. S. Kamlet, D. C. Powers, C. N. Neumann, G. B. Boursalian, T. Furuya, D. C. Choi, J. M. Hooker, T. Ritter, Science 2011, 334, 639-642.

[554] E. Lee, J. M. Hooker, T. Ritter, J. Am. Chem. Soc. 2012, 1745617458.

[*] Dr. T. Liang, C. N. Neumann, Prof. T. Ritter Department of Chemistry and Chemical Biology Harvard University

12 Oxford Street, Cambridge, MA 02138

Fax: (+1) 617-496-4591

E-mail: tliang@post.harvard.edu, cneumann@fas.harvard.edu, ritter@chemistry.harvard.edu

Homepage: http://www.chem.harvard.edu/groups/ritter/

\section{[**] ((General Annotations))}


Entry for the Table of Contents (Please choose one layout)

Layout 1:

\section{Fluorination}

T. Liang, C. N. Neumann, T. Ritter

Page - Page

Introduction of Fluorine and FluorineContaining Functional Groups

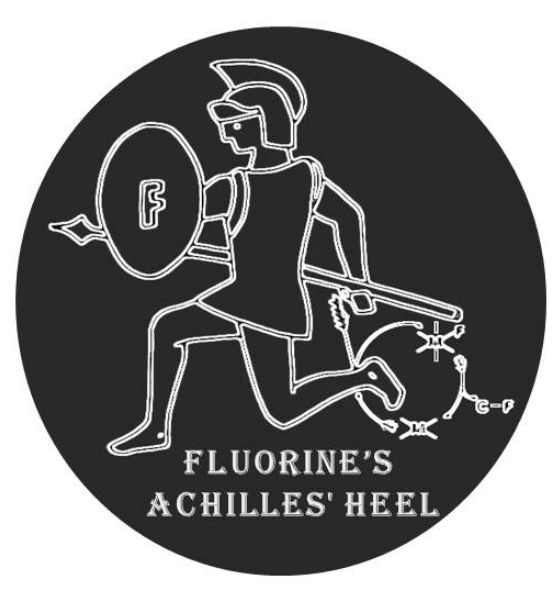

Fluorination chemistry is more than 100 years old, yet, it still remains a challenge today. Recent advances, to a large extend enabled by catalysis, have resulted in more efficient methods to introduce fluorine and fluorinecontaining functional groups into functionalized molecules. This review focuses on new strategies for fluorination, with a brief introduction to conventional fluorination, so that the modern methods can be put into perspective. 